\title{
严
}

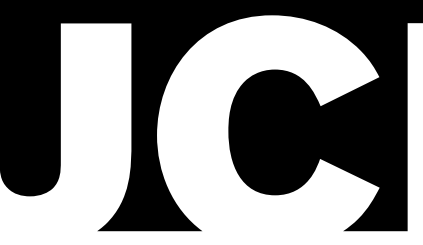

\section{Investigation into high efficiency visible light photocatalysts for water reduction and oxidation}

\section{David James Martin}

2014

\section{A thesis submitted for the partial fulfilment of the requirements for the} degree of Doctor of Philosophy at University College London

\author{
Department of Chemical Engineering, \\ University College London, \\ Torrington Place, \\ London,
}

WC1E 7JE

\section{$\overline{\text { EPSRC }}$}

Engineering and Physical Sciences Research Council 


\section{Declaration}

I, David James Martin, confirm that the work presented in this thesis is my own. Where information has been derived from other sources, I confirm that this has been indicated in the thesis.

Signature

\section{Date}




\section{Acknowledgements}

Firstly, I would like to thank my supervisor, Dr Junwang Tang for his unparalleled guidance, expertise and insight throughout the entire project. Throughout the ups and downs, he remained focused and determined, two characteristics which have definitely rubbed off on me. He will remain a lifelong mentor and friend. I would also like to thank my second supervisor, Prof. Jawwad Darr, for many beneficial discussions, and for being very supportive in difficult times.

I'm eternally grateful to Mark Turmaine and Jim Davy for helping me with SEM, Steve Firth for help with TEM/FTIR/Raman spectroscopy, Martin Vickers with XRD, and Rob Gruar for helping me with the most annoying instrument ever (ZetaSizer Nano). A special mention goes to Xiaowei Chen and the humble Juan Jose Delgado for consistently collaborating via their excellent TEM expertise.

A huge mention must go to Dr Naoto Umezawa and Prof. Jinhua Ye (National Institute of Materials Science, 'NIMS', Japan). Prof. Ye, who very kindly let me undertake a short research internship in her group, taught me in a very short space of time, to not only think outside the box, but also to make sure the box has the correct space group reflection conditions - so you know where the edges are! In my first international collaboration, Naoto was not only a good friend, but a patient and thoughtful man who had real faith that our work would be complementary and hence publishable. We had many discussions both personal and professional, and he will remain another lifelong friend. I thank them both for making my study in Japan thoroughly enjoyable.

I would also like to thank the forever enthusiastic and forward-thinking Dr Stephen Shevlin, who was heavily involved in the second collaboration. His insight into DFT and TDDFT studies complimented and explained some of the experimental work I completed on carbon nitride. On a personal level Stephen also taught me about the ups and downs of reviewers, which I'll never forget. Kaipei Qiu was also extremely helpful in contributing to a publication. Prof. Z. Xiao Guo, who co-supervised the collaboration with Dr Junwang Tang, was also precise, thoughtful and insightful throughout.

Dr Albertus Handoko and Dr Savio Moniz were two of the best post-docs a student could have. Before Albertus arrived, working without a PDRA was frankly a little difficult. Sometimes it's really beneficial to have 
somebody who can give you a quick answer, rather than search for it for hours. Definitely a friend for life, and despite him moving away from the group, I'm sure our paths will cross again soon.

The people who made my $\mathrm{PhD}$ actually fun in moments, kept me sane and helped with work during the later hours; Ben, Phil, Rhod, Seamus, Lawerence, Mayo, Amal, Chara, Noor, Mithila, Miggy, Eria, Moz, Toby, Jay, Vidal, Erik and all the rest of you guys.

To my fiancé Catariya, who was there throughout the good times and bad - I couldn't have done it without you. Finally, to my family; Mum, Dad, brothers, Nan and Granddad - you literally kept me going and I will never forget the sacrifices made. 


\section{Abstract}

Solar water splitting using an inorganic semiconductor photocatalyst is viewed as one of the most exciting and environmentally friendly ways of producing clean renewable fuels such as hydrogen from abundant resources. Currently, there are many diverse semiconductors that have been developed, the majority for half reactions in the presence of sacrificial reagents. However, for industrial facilitation, there exists an essential, nondebatable trifecta of being robust, cheap and efficient for overall water splitting. To date, no system has combined all three, with most examples missing at least one of the necessary trio. Therefore one of the current challenges of the field is to develop low cost, highly efficient and stable photocatalysts for industrial scale-up use. In order to achieve that aim, researchers must focus on novel semiconductors to improve efficiencies and also understand the fundamental mechanisms. The primary focus of this thesis then, is to investigate some of the newest photocatalysts for water photooxidation, reduction, and overall water splitting. In doing so, the thesis aids to shed light on the mechanisms behind what makes certain photocatalysts either efficient or inefficient.

Firstly, test station was set up to analyse gaseous products such as hydrogen and oxygen produced from photocatalytic water splitting, by using a custom made high purity borosilicate reactor in conjunction with a gas chromatography unit. Gaseous products could be measured with very small sampling error $(<1 \%)$, which improved the throughput of experiments.

The photooxidation of water using a novel faceted form of $\mathrm{Ag}_{3} \mathrm{PO}_{4}$ was investigated. A novel synthetic method was created that made it possible to control the exposing facets of silver phosphate in the absence of surfactants to yield tetrahedral crystals composed entirely of $\{111\}$ facets. It was found that due to high surface energy of $\{111\}$, and low hole $\left(h^{+}\right)$ mass in the $<111>$ direction, $\mathrm{Ag}_{3} \mathrm{PO}_{4}$ tetrahedral crystals could outperform all other low index facets for the oxidation of water under visible light. The quantum yield was found to be nearly unity at $400 \mathrm{~nm}$, and over $80 \%$ at $500 \mathrm{~nm}$. With the exception of $\mathrm{Ag}_{3} \mathrm{PO}_{4}$ tetrahedral crystals, no photocatalyst has exhibited quantum efficiencies reaching $100 \%$ under visible irradiation. Therefore, the strategy of morphology control of a photocatalyst, led by DFT calculations of surface energy and charge carrier mobility, in order to boost photooxidation yield has been demonstrated to 
be very successful, and could be applied to improve other semiconductors in future research.

Hydrogen production from water was further studied using the only known robust organic photocatalyst, graphitic carbon nitride $\left(\mathrm{g}^{-} \mathrm{C}_{3} \mathrm{~N}_{4}\right)$. It was discovered that using a novel preparation method, urea derived g- $\mathrm{C}_{3} \mathrm{~N}_{4}$ can achieve a quantum yield of $26 \%$ at $400 \mathrm{~nm}$ for hydrogen production from water; an order of magnitude greater than previously reported in the literature $(3.75 \%)$. The stark difference in activity is due to the polymerisation status, and consequently the surface protonation status as evidenced by XPS. As the surface protonation decreases, and polymerisation increases, the performance of graphitic carbon nitride for hydrogen production increases. The rate of hydrogen production with respect to BET specific surface area was also found to be non-correlating; a juxtaposition of conventional photocatalysts whose activity is enhanced with larger surface areas - believed to be because of an increase in surface active sites.

Finally, overall water splitting was probed using Z-scheme systems comprising of a redox mediator, hydrogen evolution photocatalyst, and oxygen evolution photocatalyst. $\mathrm{Ag}_{3} \mathrm{PO}_{4}$ was found not be not suitable for current $\mathrm{Z}$-scheme systems, as it is unstable in the $\mathrm{pH}$ ranges required, and also reacts with both of the best known electron mediators used in Zschemes, as evidenced by XRD, TEM, and EDX studies. However, it has been demonstrated that urea derived $\mathrm{g}-\mathrm{C}_{3} \mathrm{~N}_{4}$ can participate in a Z-scheme system, when combined with either $\mathrm{WO}_{3}$ or $\mathrm{BiVO}_{4}$ - the first example of its kind, resulting in a stable system for an overall water splitting operated under both visible light irradiation and full arc irradiation. Further studies shows water splitting rates are influenced by a combination of $\mathrm{pH}$, concentration of redox mediator, and mass ratio between photocatalysts. The solar-to-hydrogen conversion of the most efficient system was experimentally verified to be ca. $0.1 \%$. It is postulated that the surface properties of urea derived graphitic carbon nitride are related to the adsorption of redox ions, however, further work is required to confirm these assumptions. 


\section{Publications \& conferences}

\section{Publications}

1. Efficient visible driven photocatalyst, silver phosphate: performance, understanding and perspective. David James Martin, Liu Guigao, Jinhua Ye and Junwang Tang. Chemical Society Reviews, in preparation, 2014

2. Visible Light-Driven Pure Water Splitting by a Nature-Inspired Organic Semiconductor-Based System. David James Martin, Philip James Thomas Reardon, Savio J.A. Moniz, and Junwang Tang. Journal of the American Chemical Society, 2014, 136 (36), 12568 -12571

3. Highly Efficient $\mathbf{H}_{2}$ Evolution from Water under visible light by Structure-Controlled Graphitic Carbon Nitride. David James Martin, Kaipei Qiu, Stephen Andrew Shevlin, Albertus Denny Handoko, Xiaowei Chen, Zheng Xiao Guo \& Junwang Tang. Angewandte Chemie International Edition, 2014, 53 (35), 9240-9245

4. Facet engineered $\mathbf{A g}_{3} \mathrm{PO}_{4}$ for efficient water photooxidation. David James Martin, Naoto Umezawa, Xiaowei Chen, Jinhua Ye and Junwang Tang. Energy \& Environmental Science, 2013, 6, 3380-3386

5. $\mathrm{H}_{2}$ and $\mathrm{O}_{2}$ Evolution from Water Half-Splitting Reactions by Graphitic Carbon Nitride Materials. A. Belen Jorge, David James Martin, Mandeep T. S. Dhanoa, Aisha S. Rahman, Neel Makwana, Junwang Tang, Andrea Sella, Furio Corà, Steven Firth, Jawwad A. Darr, and Paul F. McMillan. The Journal of Physical Chemistry C, 2013, 117 (14), 7178-7185

6. Conversion of solar energy to fuels by inorganic heterogeneous systems. Kimfung Li, David James Martin and Junwang Tang. Chinese Journal of Catalysis 2011, 32 (6), 879-890

\section{Other publications}

7. $\mathrm{CuO}_{\mathrm{x}}-\mathrm{TiO}_{2}$ junction: what is the active component for photocatalytic $\mathrm{H}_{2}$ production? 
Zhonlei Wang, Yuanxu Liu, David James Martin, Wendong Wang, Junwang Tang and Weixin Huang. PCCP, 2013, 15, 14956-14960

\section{Conferences}

- "Highly Efficient Photocatalytic $\mathbf{H}_{2}$ Evolution from Water using Visible Light and Structure-Controlled Graphitic Carbon Nitride", Materials for Solar-Driven Fuels Synthesis (Royal Society satellite meeting), Solar Fuels Network, London, UK, July 2014

- "Facet engineered $\mathrm{Ag}_{3} \mathrm{PO}_{4}$ for efficient water photooxidation", International Symposia on Advancing the Chemical Sciences ('ISACS 12'), RSC, Cambridge, UK, September 2013

- "Facet engineered $\mathrm{Ag}_{3} \mathrm{PO}_{4}$ for efficient water photooxidation", Solar Fuel 13, NanoGe, Granada, Spain, June 2013.

\section{Contents}

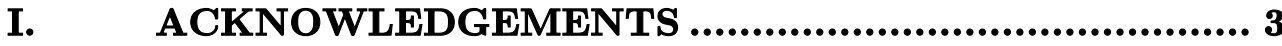

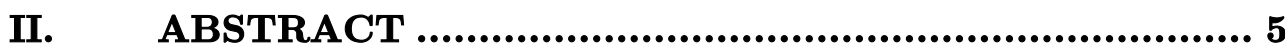

III. PUBLICATIONS \& CONFERENCES......................... 7





VI. LIST OF EQUATIONS .....................................17

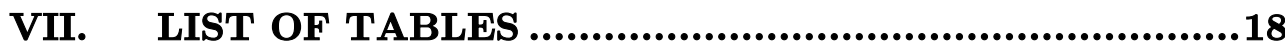

VIII. NOMENCLATURE..............................................19

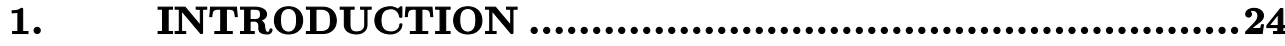


2.1 Fundamentals of semiconductor photoelectrochemistry..28

2.1.1 Semiconductor-electrolyte interface .......................... 28

2.1.2 Charge carrier generation........................................ 29

2.1.3 Photoelectrochemistry ............................................ 32

2.1.4 Photocatalytic water splitting ..................................... 35

2.1.5 Efficiency calculations............................................. 38

2.1.6 Thermodynamic limits............................................. 40

2.2 Characterisation methods for photocatalysts .................43

2.2.1 UV-Visible spectroscopy ........................................ 44

2.2.2 Gas Chromatography ............................................... 45

2.2.3 Powder X-Ray Diffraction (PXRD) .......................... 46

2.2.4 Scanning and Transmission Electron Microscopy .......... 47

2.2.5 TGA-DSC-MS ....................................................... 48

2.2.6 BET method for specific surface area measurements ${ }^{50} \ldots 49$

2.2.7 Zeta Potential (ZP) using Electrophoretic Light

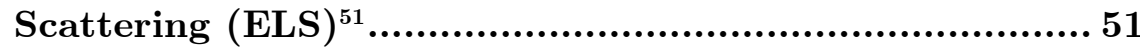

2.2.8 Attenuated Total Reflectance - Fourier Transform

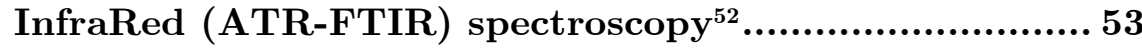

2.2.9 Raman Spectroscopy ............................................... 54

2.2.10 X-ray Photoelectron Spectroscopy (XPS) ................... 56

2.2.11 Elemental Analysis (EA) ...................................... 58

2.3 Overview of current photocatalysts ${ }^{66} \ldots \ldots \ldots \ldots \ldots \ldots \ldots \ldots \ldots \ldots . . . . .59$

2.3.1 UV-active semiconductors ...................................... 59

2.3.2 Semiconductors activated by visible light .....................65

2.3.3 Z-Scheme systems .................................................. 73

2.3.4 Effect of morphology, crystallinity and size on the activity of photocatalysts................................................. 78

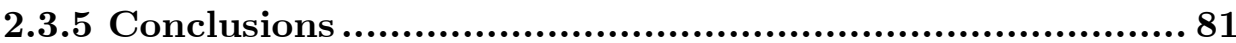

3. EXPERIMENTAL DEVELOPMENT ......................82

3.1 Reaction system ........................................................82

3.1.1 Reactor ............................................................... 83

3.1.2 Light Source.............................................................. 85 


\subsection{GAS CHROMATOGRAPHY: SELECTION AND}

CALIBRATION ...................................................................86

3.2.1 Gas Chromatography setup.......................................86

3.2.2 Standard gas and calibration....................................88

3.3 GENERAL CHARACTERISATION ......................93

3.3.1 UV-Vis Spectrophotometry ....................................93

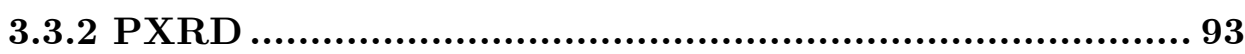

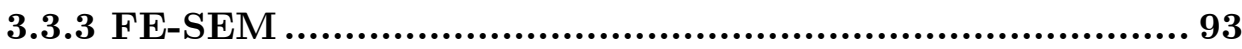

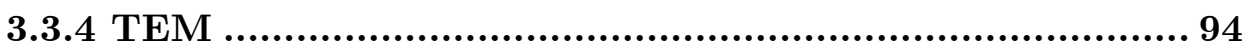

3.3.5 BET specific surface area ....................................... 94

3.3.6 ATR-FTIR spectroscopy .................................... 94

3.3.7 Raman spectroscopy .............................................. 94

3.3.8 TGA-DSC-MS ................................................... 94

3.3.9 Zeta Potential (ZP) measurements ............................. 95

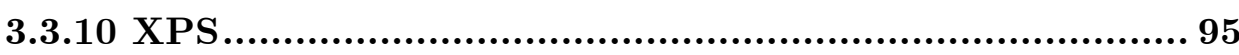

3.3.11 Elemental Analysis .............................................. 95

4. OXYGEN EVOLVING PHOTOCATALYST DEVELOPMENT ...........................................................96



4.2 Methodology ..........................................................98

4.2.1 Photocatalytic analysis ........................................ 98

4.2.2 Synthesis techniques ......................................... 99

4.3 Results and discussion........................................... 103

4.3.1 Initial $\mathrm{Ag}_{3} \mathrm{PO}_{4}$ studies ..................................... 103

4.3.1.1 Method 'A' ...................................................... 103

4.3.1.2 Method 'B' .................................................... 106

4.3.1.3 Method ' $C$ ' ................................................... 110

4.3.2 Facet control of $\mathrm{Ag}_{3} \mathrm{PO}_{4}\left(\operatorname{method}\right.$ ' $\mathrm{D}$ ') ${ }^{213} \ldots \ldots \ldots \ldots \ldots \ldots \ldots . . . . . .112$

4.4 Conclusions ........................................................ 127 
5. HYDROGEN EVOLVING PHOTOCATALYST

DEVELOPMENT ......................................................... 130

5.1 Introduction .................................................... 131

$5.2 \quad$ Methodology ................................................... 132

5.2.1 Photocatalytic analysis ...................................... 132

5.2.2 Synthesis techniques........................................... 133

$5.3 \quad$ Results and discussion.......................................... 134

$5.4 \quad$ Conclusions ..................................................... 159

6. NOVEL Z-SCHEME OVERALL WATER SPLITTING SYSTEMS ..................................................................161

6.1 Introduction .................................................. 162

6.2 Methodology ........................................................ 162

6.2.1 Photocatalytic analysis ........................................ 162

6.2.2 Synthesis techniques ........................................ 163

6.3 Results and discussion......................................... 165

6.3.1 $\mathrm{Ag}_{3} \mathrm{PO}_{4}$ based Z-scheme water splitting systems.......... 165

6.3.2 Graphitic carbon nitride based Z-scheme water splitting

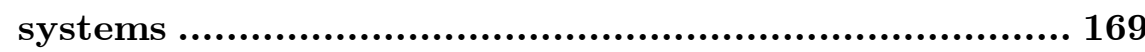

6.4 Conclusions ........................................................ 188

7. OVERALL CONCLUSIONS ................................. 190

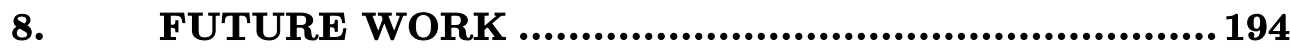

9. BIBLIOGRAPHY ......................................... 196 


\section{List of Figures}

Figure 1. An example of an n-type semiconductor in contact with an electrolyte - (a) before equilibrium, (b) after equilibrium. Adapted from references ${ }^{15,20}$. Symbols are as follows: $\mathrm{E}_{\mathrm{CB}}-$ bottom of a conduction band, $E_{\mathrm{VB}}$ - top of a valence band, $\mathbf{E}_{\mathrm{BG}}-$ band gap, $E_{F}-$ Fermi level, $d_{s c}-$ depth of space charge region. ......... 28 Figure 2. Idealised diagram of charge separation in a semiconductor photocatalyst.

Figure 3. Positions (black) and magnitudes (blue) of the most well researched semiconductors (red). The green region represents the redox minimum between 0 and $1.23 \mathrm{~V}$ vs NHE. Reproduced from reference ${ }^{26}$

Figure 4. A typical PEC cell for photoelectrochemistry, with applied bias to drive proton reduction. Inspired by Kudo and Miseki ${ }^{23}$

Figure 5. An illustration of the three main processes in photocatalytic water splitting. Adapted from reference ${ }^{23}$....

Figure 6. The maximum photoconversion efficiency possible as a function of the band gap wavelength $(150 \mathrm{~W} \mathrm{Xe})$. Indicated with a dashed line is the maximum band gap wavelength at $610 \mathrm{~nm}$. This corresponds with a minimum band gap of $2.03 \mathrm{eV}$ for water splitting. Reproduced from reference ${ }^{15}$.

Figure 7. Schematic demonstration of a working ATR crystal in an ATR-FTIR spectrometer ${ }^{52}$.......................................... 54 Figure 8. Raleigh and Stokes/Anti Stokes scattering processes in diagram form. Lowest energy vibrational state is $\mathbf{n}_{0}$, and highest virtual states is $v_{2}$. Arrows represent direction of photon excitation (upwards) and relaxation (downwards).... 55

Figure 9. Formation of new valence and conduction bands by electron donor and acceptor atoms, enabling a UV-active material to respond to visible light.......................................................66 Figure 10. Illustration of GaN: $\mathrm{ZnO}$ solid solution. Conduction band orbitals remain as $\mathrm{Ga} 4 \mathrm{~s} 4 \mathrm{p}$, whilst valence band is hybridised to N2p, Zn3d and O2p. Adapted from Maeda et al ${ }^{132}$.

Figure 11. Ball \& stick (left), and molecular schematic drawing of g- $\mathrm{C}_{3} \mathrm{~N}_{4}$ (right), using ChemSketch ${ }^{\odot}$. A single heptazine unit is highlighted in red. Graphitic carbon nitride is composed of; carbon (grey), nitrogen (blue), and hydrogen atoms (white)..... 72 Figure 12. (A) Natural photosynthesis (B) An artificial analogy involving two different photocatalysts possessing two different band positions ( $\mathrm{A}$ is electron acceptor, $\mathrm{D}$ is electron donor) ${ }^{172} \ldots 74$ Figure 13. Schematic of proposed reactor (A) without magnetic stirring, (B) with stirring. ................................................... 82 Figure 14. Borosilicate reactor for water splitting batch reactions.

Figure 15. Photo.....................................................84 photocatalysis experiments. ...............................................8 86 
Figure 16. Idealised example chromatogram. A baseline is indicated by a horizontal red line, and integrated area under the curve (in $\mu \mathrm{V} . \mathrm{min}$ ) is indicated by a shaded blue area............... 88 Figure 17. GC area vs. molar amount calibration curves for (A) hydrogen and (B) oxygen. Equations of linear fits and $R^{2}$ values are noted in the upper left hand corner of the figure. .............. 90 Figure 18. SEM micrographs of samples A1 (A \& B), A2 (C \& $\mathrm{D})$, and $\mathrm{A3}(\mathrm{E} \& \mathrm{~F})$.

Figure 19. (A) XRD patterns of samples A1, A2 and A3. (B) UVVis absorbance spectra of samples A1, A2, and A3............... 105

Figure 20. Oxygen evolution under visible light $(\lambda>420 \mathrm{~nm})$ from water, in the presence of $\mathrm{AgNO}_{3}(0.85 \mathrm{~g})$, at neutral $\mathrm{pH}$, using

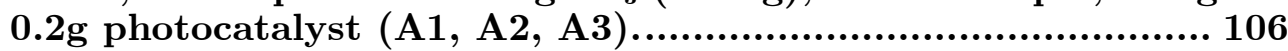
Figure 21. SEM micrographs of samples B1 (A \& B), B2 (C \& $\mathrm{D})$, and $\mathrm{B3}$ (E)... 107

Figure 22. XRD patterns for samples B1, B2 and B3. The standard ISCD/JCPDS pattern for $\mathrm{Ag}_{3} \mathrm{PO}_{4}$ is shown as red bars. The Bragg peaks of the unknown phase are indicated by the symbol ' $\otimes$ '. 108 Figure 23. UV-Vis absorbance spectra for samples B1, B2 and B3.

Figure 24. Oxygen evolution under visible light $(\lambda>420 \mathrm{~nm})$ from water, in the presence of $\mathrm{AgNO}_{3}(0.85 \mathrm{~g})$, at neutral $\mathrm{pH}$, using $0.2 \mathrm{~g}$ photocatalyst (B1, B2, B3). 109 Figure 25. SEM micrographs of sample $\mathrm{C} 1$ in low (A) and high (B) resolution. 110

Figure 26. UV-Vis absorbance spectra of sample C1. The grey area visually demonstrates the range of the $420 \mathrm{~nm}$ long pass filter. A black line indicates the band edge and approximate absorption limit.

Figure 27. XRD pattern for sample C1. The standard

ISCD/JCPDS pattern for $\mathrm{Ag}_{3} \mathrm{PO}_{4}$ is shown as red bars, and the corresponding miller indices of each plane are indicated above each peak.

Figure 28. Oxygen evolution under visible light $(\lambda>420 \mathrm{~nm})$ from water, in the presence of $\mathrm{AgNO}_{3}(0.85 \mathrm{~g})$, at neutral $\mathrm{pH}$, using $0.2 \mathrm{~g}$ photocatalyst $(\mathrm{C} 1)$. Indicated are the regions of most importance; the initial period where $\mathrm{O}_{2}$ evolution rate is high (rates are calculated from this point), and where the rate deviates from linearity in the later stages of the experiment................. 112 Figure 29. Relaxed geometries for (a) $\{100\}$, (b) $\{110\}$, and (c) \{111\} surface of $\mathrm{Ag}_{3} \mathrm{PO}_{4}$ (calculations performed by Naoto Umezawa, NIMS $)^{213}$

Figure 30. (A) $\mathrm{Ag}_{3} \mathrm{PO}_{4} 2 \times 2 \times 2$ supercell and (B) $\mathrm{Ag}_{3} \mathrm{PO}_{4}$ tetrahedron cell (Figure from Martin et $\mathrm{al}^{213}$ )......................... 115 Figure 31. SEM micrographs of silver phosphate cubic crystals (A and $B$ ), and rhombic dodecahedral crystals (C and $D) \ldots \ldots \ldots \ldots . . .116$ Figure 32. Low $(A)$ and high $(B, C)$ magnification SEM micrographs of $\mathrm{Ag}_{3} \mathrm{PO}_{4}$ tetrahedrons $\left(20 \mathrm{~cm}^{3} \mathrm{H}_{3} \mathrm{PO}_{4}\right)$. Low (D) and high (E) magnification SEM micrographs of $\mathrm{Ag}_{3} \mathrm{PO}_{4}$ 
tetrapods synthesised using $2 \mathrm{~cm}^{3} \mathrm{H}_{3} \mathrm{PO}_{4}$. (F) SEM micrographs illustrating the formation of $\mathrm{Ag}_{3} \mathrm{PO}_{4}$ tetrahedrons from tetrapods by varying the concentration of $\mathrm{H}_{3} \mathrm{PO}_{4}$ (left to right; 2, 5, 10, 15,

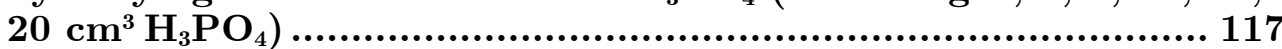

Figure 33. TEM micrograph tilt study, performed by collaborator, Xiaowei Chen. A tetrahedron indicated by dotted lines is rotated on axis from -66 to $+50^{\circ}$.

Figure 34. XRD pattern of faceted $\mathrm{Ag}_{3} \mathrm{PO}_{4}$ crystals. ............... 119 Figure 35. UV-Vis absorbance spectra of faceted $\mathrm{Ag}_{3} \mathrm{PO}_{4}$ crystals.

Figure 36. Particle diameter histograms for $\mathbf{A g}_{3} \mathbf{P O}_{4}(\mathbf{A})$ tetrahedrons, (B) cubes, (C) rhombic dodecahedrons. $300 \mathrm{~W}$ Xe light source under full arc irradiation, with $\mathrm{AgNO}_{3}$ acting as an electron scavenger. (B) Oxygen yield comparison between tetrahedral $\mathrm{Ag}_{3} \mathrm{PO}_{4}$, previously reported random mixed faceted $\mathrm{Ag}_{3} \mathrm{PO}_{4}$ and $\mathrm{BiVO}_{4}$ under $300 \mathrm{~W}$ Xe lamp full arc irradiation, using $\mathrm{AgNO}_{3}$ as an electron scavenger. (C) Oxygen evolution of tetrahedral crystals using a $300 \mathrm{~W}$ Xe lamp fitted with $420 \mathrm{~nm}$ long pass filter, $\mathrm{AgNO}_{3}$ was used as an electron scavenger $(0.85 \mathrm{~g})$. The mixed facet sample was synthesised using method $\mathrm{C}$ and $\mathrm{Na}_{2} \mathrm{HPO}_{4} \cdot \mathrm{BiVO}_{4}$ was synthesised according to a previously reported recipe ${ }^{17}$

Figure 38. BET adsorption isotherms for $\mathrm{Ag}_{3} \mathrm{PO}_{4}$ tetrahedrons, cubes, and rhombic dodecahedrons. ..................................123

Figure 39. XRD pattern before and after a full arc photocatalytic test for the photooxidation of water by tetrahedral $\mathrm{Ag}_{3} \mathrm{PO}_{4}$.

$\mathrm{AgNO}_{3}$ was used as a scavenger, and thus Ag metal deposited on the surface (JCPDS 00-04-0783).

Figure 40. (A) Oxygen evolution using a $400 \mathrm{~nm}$ band pass filter, and (B) Internal quantum yield variation using different band pass filters, using $\mathrm{AgNO}_{3}$ as a scavenger, and a $300 \mathrm{~W}$ Xe light source.

Figure 41. XRD patterns of $\mathrm{g}-\mathrm{C}_{3} \mathrm{~N}_{4}$ synthesised using different precursors using $5{ }^{\circ} \mathrm{C}$ ramp rate, $600{ }^{\circ} \mathrm{C}$, 4 hours hold time.... 135 Figure 42. ATR-FTIR spectra of powdered graphitic carbon nitride; annotated are characteristic bands for $\mathrm{g}-\mathrm{C}_{3} \mathrm{~N}_{4}{ }^{229} \ldots \ldots \ldots 136$ Figure 43. UV-Vis absorbance spectra for graphitic carbon nitride powders synthesized using different precursors at $600{ }^{\circ} \mathrm{C} . . . . . . .137$ Figure 44. Raman spectra of different carbon nitride samples. 137 Figure 45. TEM micrographs of $\mathrm{g}-\mathrm{C}_{3} \mathrm{~N}_{4}$ synthesised from: (A) Urea, (B) Thiourea, and (C) DCDA. The micrograph scheme at (D) shows a continual magnification of the $\mathrm{g}-\mathrm{C}_{3} \mathrm{~N}_{4}$ (urea) structure, and the resulting diffraction rings from microcrystalline regions

Figure 46. BET nitrogen adsorption/desorption isotherms. All samples were pre-prepared by calcination at $600{ }^{\circ} \mathrm{C}$, for 4 hours, with a ramp rate of $5{ }^{\circ} \mathrm{C} /$ minute.

Figure 47. TGA-DSC-MS data from $\mathrm{g}-\mathrm{C}_{3} \mathrm{~N}_{4}$ synthesised from urea and thiourea. 
Figure 48. TGA (A) and DSC (B) analysis of urea and thiourea.

Figure 49. Hydrogen evolution using a $300 \mathrm{~W}$ Xe lamp, $3 \mathrm{wt \%}$ 142

Pt, TEOA as a hole scavenger; (A) Full arc, (B) $\lambda \geq 395 \mathrm{~nm} . . .142$

Figure 50. Hydrogen production with various synthesis parameters of urea derived $\mathrm{g}-\mathrm{C}_{3} \mathrm{~N}_{4}(\lambda \geq 395 \mathrm{~nm}$, TEOA hole scavenger, $300 \mathrm{~W}$ Xe lamp). (A) Urea based $\mathrm{g}-\mathrm{C}_{3} \mathrm{~N}_{4}$ synthesized at different temperatures, $4 \mathrm{~h}$ hold time $\left({ }^{\circ} \mathrm{C}\right)$. (B) Urea synthesized at $600{ }^{\circ} \mathrm{C}$ using different ramping temperatures $\left({ }^{\circ} \mathrm{C} /\right.$ minute). (C) g- $\mathrm{C}_{3} \mathrm{~N}_{4}$ (urea) with different cocatalysts, $3 \mathrm{wt} \%$. (D) Weight percent of $\mathrm{Pt}$ in comparison to photocatalyst (urea, $\left.600{ }^{\circ} \mathrm{C}\right)$.

Figure 51. XPS spectra from $\mathrm{g}-\mathrm{C}_{3} \mathrm{~N}_{4}$, for urea, DCDA and thiourea derived samples.

Figure 52. C1s and O1s XPS spectra of $\mathrm{g}-\mathrm{C}_{3} \mathrm{~N}_{4}$ synthesised from different precursors........................................................... 148

Figure 53. Ratios of bonds within the N1s core level peak in different samples and their comparisons to the HER under visible light $(\lambda \geq 395 \mathrm{~nm})$, indicating decreasing proton concentration dramatically increases photocatalytic activity........................ 149 Figure 54. Zeta potential of $\mathrm{g}-\mathrm{C}_{3} \mathrm{~N}_{4}$ synthesised from different precursors $\left(600{ }^{\circ} \mathrm{C}\right)$

Figure 55. Correlation between specific surface area normalised hydrogen evolution rate and bulk hydrogen content measured by elemental analysis.

Figure 56. Geometric and electronic structure of single sheet g$\mathrm{C}_{3} \mathrm{~N}_{4}$. (a) Supercell model of sheet carbon nitride, (b) supercell model of protonated carbon nitride. Nitrogen is denoted by blue spheres, carbon brown spheres, and hydrogen light pink spheres. (c) Total density of states for sheet carbon nitride (black line) and protonated carbon nitride (red line). Energy is with respect to the zero of the sheet carbon nitride simulation. The DOS of the protonated carbon nitride has been shifted so that the corresponding zero points align.

Figure 57. Total and site-decomposed DOS for protonated carbon nitride model. The strong contribution of the carbon atoms adjacent to the N3c' site at the CBE is apparent, as the six atoms contribute almost as much to the DOS as the other twelve carbon atoms in the supercell. 154

Figure 58. Excited state properties of bare (sheet) and protonated carbon nitride; (a) Optical properties of $\mathrm{C}_{18} \mathrm{~N}_{28} \mathrm{H}_{12}$ (black line) and $\mathrm{C}_{18} \mathrm{~N}_{28} \mathrm{H}_{13}$ (red line) clusters, the $\mathrm{x}$-axis is labeled with respect to the vacuum level $(\mathrm{E}=0)$ while dashed lines indicate the positions of the HOMO states of the two clusters, (b) lowest energy exciton of $\mathrm{C}_{18} \mathrm{~N}_{28} \mathrm{H}_{12}$ cluster, (c) lowest energy exciton of $\mathrm{C}_{18} \mathrm{~N}_{28} \mathrm{H}_{13}$ cluster. Orange isosurface indicates distribution of photohole upon photoexcitation, green isosurface indicates distribution of photoelectron upon photoexcitation. Isosurfaces were plotted at 
$0.005|\mathrm{e}| / \AA^{3}$. Blue spheres denote hydrogen atoms, grey spheres denote carbon atoms, white spheres denote hydrogen atoms. .. 154 Figure 59. (a) Quantum yield of urea based g- $\mathrm{C}_{3} \mathrm{~N}_{4}$; using band pass filters at specific wavelengths (black line represents absorbance, internal quantum yield is shown by blue points). (b) Stability test of the urea derived g- $\mathrm{C}_{3} \mathrm{~N}_{4}$ under visible light irradiation $(\lambda \geq 395 \mathrm{~nm})$

Figure 60. (A) SEM micrograph of $\mathrm{g}_{-} \mathrm{C}_{3} \mathrm{~N}_{4}$-MCA-DMSO-30-600. (B) UV-Vis absorbance spectra of $\mathrm{g}-\mathrm{C}_{3} \mathrm{~N}_{4}$-MCA-DMSO-30-600.

Figure 61. Powder X-ray diffraction data post water splitting in g- $\mathrm{C}_{3} \mathrm{~N}_{4}-\mathrm{Fe}^{2+/ 3+}-\mathrm{Ag}_{3} \mathrm{PO}_{4}$ system. Both main phase silver phosphate and sub phase silver sulphate are indicated by red and blue bars respectively. 165

Figure 62. (A) TEM micrographs of $\mathrm{Ag}_{3} \mathrm{PO}_{4}$ with $\mathrm{AgI}$ layer post water splitting, in $\mathrm{g}-\mathrm{C}_{3} \mathrm{~N}_{4}-\mathrm{I}^{-} / \mathrm{IO}_{3}{ }^{-}-\mathrm{Ag}_{3} \mathrm{PO}_{4}$ system. Atomic spaces indicated by parallel lines. (B) Corresponding FFT of micrograph, diameter of diffraction spots are indicated........... 166 Figure 63. (A) TEM micrograph of an $\mathrm{Ag}_{3} \mathrm{PO}_{4}$ crystal post water splitting, in $\mathrm{g}-\mathrm{C}_{3} \mathrm{~N}_{4}-\mathrm{I}^{-} / \mathrm{IO}_{3}{ }^{-}-\mathrm{Ag}_{3} \mathrm{PO}_{4}$ system. Atomic plane distance indicated by parallel lines. (B) Corresponding FFT of micrograph, diameter of diffraction spot is indicated. Figure 64. TEM micrographs (low res mode) combined with EDX of $\mathrm{Ag}_{3} \mathrm{PO}_{4}-\mathrm{AgI}$ post water splitting in $\mathrm{g}-\mathrm{C}_{3} \mathrm{~N}_{4}-\mathrm{I}^{-} / \mathrm{IO}_{3}{ }^{-}-\mathrm{Ag}_{3} \mathrm{PO}_{4}$ system. (A) Full spectrum mapping of TEM-EDX micrograph ( $\mathrm{Ag}=$ green, $\mathrm{O}=$ pink, $\mathbf{P}=$ yellow, $\mathrm{I}=$ blue $)$. (B) Iodine mapping of TEM-EDX micrograph. (C) EDX spectrum of corresponding TEM micrograph area $(5 \times 5 \mu \mathrm{m})$, inset denotes weight \% of map sum spectrum. Note: $\mathrm{Cu}$ signal is from the copper/holey carbon grid used as a support, and other heavy elements such as $\mathrm{Ca}$, Si, Co, Os (all 0 wt\%) are artefacts. ....... 169 Figure 65. (A) Powder XRD pattern and (B) UV-Vis absorbance spectra of $\mathrm{g}-\mathrm{C}_{3} \mathrm{~N}_{4}, \mathrm{WO}_{3}$ and $\mathrm{BiVO}_{4}$ compounds prior to water splitting reactions 170 Figure 66. SEM and TEM micrographs of different photocatalyst. (A) \& (B): Commercial $\mathrm{WO}_{3}$ crystals. (C) Synthesized $\mathrm{BiVO}_{4}$ crystals. (D) \& (E) Synthesized $g-\mathrm{C}_{3} \mathrm{~N}_{4}$ sheets....................... 171 Figure 67. Optimisation of water splitting rates using a g- $\mathrm{C}_{3} \mathrm{~N}_{4}$ $\mathrm{BiVO}_{4} \mathrm{Z}$-scheme system with $\mathrm{FeCl}_{2}$ as a redox mediator. (A) pH modification, (B) differing concentration of $\mathrm{FeCl}_{2},(\mathrm{C})$ alternating

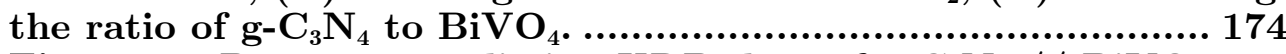
Figure 68. Post water splitting XRD data of $\mathrm{g}-\mathrm{C}_{3} \mathrm{~N}_{4} / / \mathrm{BiVO}_{4}$ system at different $\mathrm{pH}$ levels. Tetragonal phase BiOCl, JCPDS 00-006-0249, monoclinic phase $\mathrm{BiVO}_{4}$, JCPDS 00-044-0081..... 175 Figure 69. Stoichiometric water splitting (300 W Xe lamp source): (A) $\mathrm{g}-\mathrm{C}_{3} \mathrm{~N}_{4}(3 \mathrm{wt} \% \mathrm{Pt})-\mathrm{FeCl}_{2}-\mathrm{BiVO}_{4}$ under full arc irradiation, $\mathrm{pH} \mathrm{3,1:1}$ photocatalyst weight ratio, $2 \mathrm{mM} \mathrm{FeCl}$.

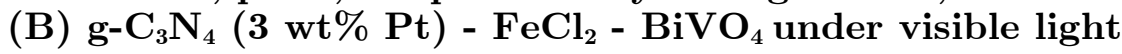
irradiation $(\lambda>395 \mathrm{~nm}), \mathrm{pH} 3,1: 1$ photocatalyst weight ratio, 2




Figure 70. Water splitting using (A) g- $\mathrm{C}_{3} \mathrm{~N}_{4}(3 \mathrm{wt} \% \mathrm{Pt})-\mathrm{NaI}-$ $\mathrm{WO}_{3}(0.5 \mathrm{wt} \% \mathrm{Pt})$ under full arc irradiation, $\mathrm{pH}$ 8.3, 1:1 photocatalyst weight ratio, $5 \mathrm{mM} \mathrm{NaI}$ and, (B) $\mathrm{g}-\mathrm{C}_{3} \mathrm{~N}_{4}(3 \mathrm{wt} \%$ $\mathrm{Pt})$ - $\mathrm{NaI}-\mathrm{WO}_{3}(0.5 \mathrm{wt} \% \mathrm{Pt})$ under visible light irradiation $(\lambda>$ $395 \mathrm{~nm}$ ), pH 8.3, 1:1 photocatalyst weight ratio, $5 \mathrm{mM}$ NaI.... 178 Figure 71. Optimisation of g- $\mathrm{C}_{3} \mathrm{~N}_{4}-\mathrm{WO}_{3} \mathrm{Z}$-scheme system with $\mathrm{NaI}$ as a redox mediator. (A) pH modification, (B) differing concentration of $\mathrm{NaI},(\mathrm{C})$ alternating the ratio of $\mathrm{g}-\mathrm{C}_{3} \mathrm{~N}_{4}$ to $\mathrm{WO}_{3}$

Figure 72. Post XRD diffraction patterns from two different $g$ $\mathrm{C}_{3} \mathrm{~N}_{4}$ based water splitting systems...................................... 181 Figure 73. BET isotherms of Commercial $\mathrm{WO}_{3}\left(\mathrm{SSA}=1.15 \mathrm{~m}^{2} \mathrm{~g}\right.$ $\left.{ }^{1}\right), \mathrm{BiVO}_{4}\left(\mathrm{SSA}=3.91 \mathrm{~m}^{2} \mathrm{~g}^{-1}\right)$, and $\mathrm{WO}_{3}$ platelets $\left(2.45 \mathrm{~m}^{2} \mathrm{~g}^{-1}\right) \ldots 181$ Figure 74. Comparison of XRD diffraction patterns of $\mathrm{WO}_{3}$ commercial crystals and as synthesised $\mathrm{WO}_{3}$ platelets.............. 183 Figure 75. Low (A) and high (B) SEM micrographs of platelet

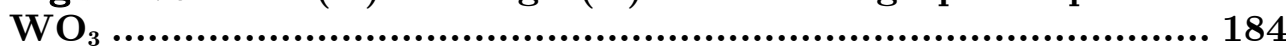
Figure 76. (A) UV-Vis absorption spectra of commercial $\mathrm{WO}_{3}$, and platelet $\mathrm{WO}_{3}$ particles, Tauc plots of; $\mathrm{WO}_{3}$ platelets $(\mathrm{B} \& \mathrm{C})$, and $\mathrm{WO}_{3}$ commercial particles $(\mathrm{C} \& \mathrm{D})$.

\section{List of equations}

Equation 1. General reaction for water splitting....................... 25

Equation 2................................................................. 30

Equation 3...................................................................... 30

Equation 4. Basic anodic reaction ....................................... 33

Equation 5. Basic cathodic reaction ..................................... 33

Equation 6. Acidic anodic reaction ....................................... 33

Equation 7. Acidic cathodic reaction ..................................... 33

Equation 8. Internal Quantum yield....................................... 39

Equation 9. Turnover Number ......................................... 39

Equation 10. STH\% ......................................................... 39

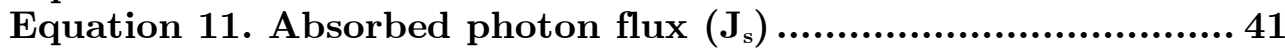

Equation 12. Limits of photoconversion efficiency $\left(\eta_{c}\right) \ldots \ldots . . . \ldots . . .41$

Equation 13. Proportion of absorbed photons converted into conduction band electrons $\left(\Phi_{\text {loss } \mathrm{P}}\right)$ : ..................................... 41

Equation 14. Tauc relation ................................................. 44

Equation 15. Kubelka-Mulk relation .................................... 44

Equation 16. Transmission-reflectance-absorption relation .........44

Equation 17. Absorbance conversion ...................................... 45

Equation 18. Planck relation $(\mathrm{nm})$.................................... 45

Equation 19. Scherrer's equation ...................................... 47

Equation 20. The BET equation .............................................. 49

Equation 21. The BET constant (c) .................................... 49

Equation 22............................................................... 50

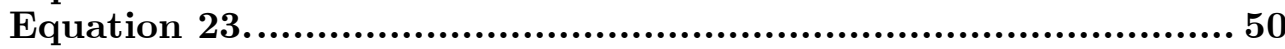


Equation 24. BET SSA equation ...................................... 50

Equation 25.............................................................. 52

Equation 26.............................................................. 52

Equation 27:.............................................................. 52

Equation 28. Conservation of energy …................................. 57

Equation 29. $\mathrm{Fe}^{2+/ 3+}$ redox potentials................................. 75

Equation 30. $\mathrm{I}^{-} / \mathrm{IO}_{3}{ }^{-}$redox potentials ................................ 75

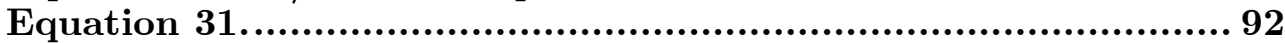

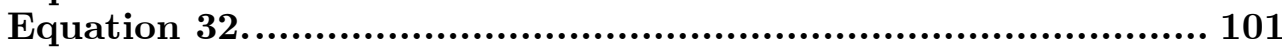

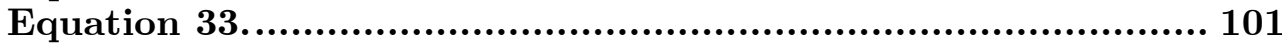

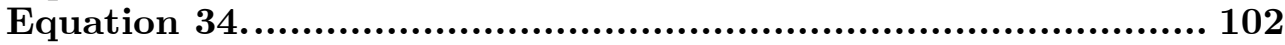

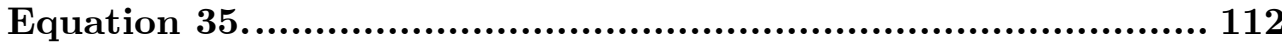

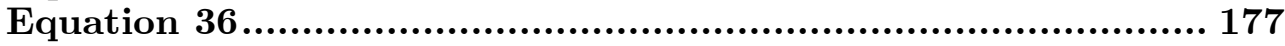

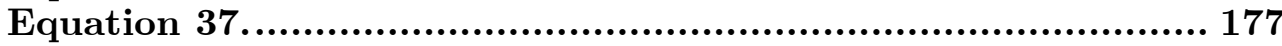

Equation 38................................................................. 177

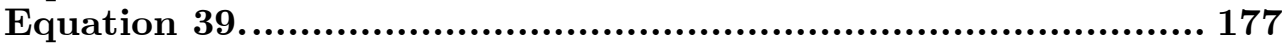



\section{List of tables}

Table 1. Summary of water splitting systems using a Z-scheme setup, currently reported in the literature ${ }^{181}$.............................77

Table 2. Gases and corresponding thermal conductivity at $\mathbf{S T P} \mathbf{P}^{211}$.

Table 3. Area sampling data for $\mathrm{H}_{2}$ and $\mathrm{O}_{2}\left(0.5 \mathrm{~cm}^{3}\right)$. SD was taken for entire population ............................................... 89

Table 4. Data table for Figure 17........................................ 91

Table 5. Summary of results on initial $\mathrm{Ag}_{3} \mathrm{PO}_{4}$ studies using various phosphate precursors. $\mathrm{All} \mathrm{Ag}_{3} \mathrm{PO}_{4}$ compounds were synthesised using $\mathrm{AgNO}_{3}$ as the silver ion source. .................. 100

Table 6. Properties of different $\mathrm{Ag}_{3} \mathrm{PO}_{4}$ crystals.................... 114

Table 7. Size, range, and standard deviation (S.D.) of various

$\mathrm{Ag}_{3} \mathrm{PO}_{4}$ crystals. Mean, mode, range, and S.D. is calculated for

100 particles using ImageJ................................................ 120

Table 8. Table showing the actual wt\% of some metal precursors.

Table 9. Summary of g- $\mathrm{C}_{3} \mathrm{~N}_{4}$ properties synthesised from different precursors. $\mathrm{H}_{2}$ evolution rate is using a $300 \mathrm{~W}$ Xe lamp, $3 \mathrm{wt} \%$ $\mathrm{Pt}$, TEOA as a hole scavenger. All samples where synthesised at $600{ }^{\circ} \mathrm{C}$ in air. TON is calculated with respect to Pt catalyst.... 143 Table 10. Ratios of bonds within the N1s core level peak in different samples and their comparisons to the hydrogen evolution activity.

Table 11. Percentage breakdown of different bonds within the

N1s spectrum................................................................ 149

Table 12. Comparison of typical g- $\mathrm{C}_{3} \mathrm{~N}_{4}$ photocatalysts reported for hydrogen production and the corresponding quantum yields. 
Table 13. AgI FFT diameter relation to a real distance.

Table 14. $\mathrm{Ag}_{3} \mathrm{PO}_{4}$ FFT diameter relation to a real distance...... 168

Table 15. Overall water splitting under full arc irradiation (300

W Xe lamp) using different redox mediated systems

Table 16. Intensity ratios of $\mathrm{WO}_{3} \mathrm{PXRD}$ Bragg peaks

\section{Nomenclature}

Acronyms \& initialisms

$\begin{array}{ll}\text { AM1.5 } & \text { Air Mass 1.5 } \\ \text { AQY } & \text { Apparent Quantum Yield } \\ \text { ATR-FTIR } & \text { Attenuated Total Reflectance Fourier Transform } \\ \text { BCC } & \text { Body Centred Cubic } \\ \text { BET } & \text { Brenauer-Emmett-Teller } \\ \text { CB } & \text { Conduction Band } \\ \text { CBE } & \text { Conduction Band Edge } \\ \text { CCD } & \text { Charge-Coupled Device camera sensor } \\ \text { CHNX } & \text { Carbon Hydrogen Nitrogen Unknown (analysis) } \\ \text { DCDA } & \text { Dicyandiamide } \\ \text { DFT (+U) } & \text { Density Functional Theory (+ Hubbard term 'U') } \\ \text { DI } & \text { Deionised (water) } \\ \text { DMSO } & \text { Dimethyl sulfoxide } \\ \text { DOS } & \text { Density Of States } \\ \text { DSC } & \text { Differential Scanning Calorimetry } \\ \text { EA } & \text { Elemental Analysis } \\ \text { EDX } & \text { Energy Dispersive X-ray Spectroscopy } \\ \text { ELS } & \text { Electrophoretic Light Scattering } \\ \text { EM } & \text { Electromagnetic } \\ \text { EtOH } & \text { Ethanol } \\ \text { FFT } & \text { Fast Fourier Transform } \\ \text { FID } & \text { Flame Ionisation Detector } \\ \text { GC } & \text { Gas Chromatography } \\ \text { HER } & \text { Hydrogen Evolution Reaction } \\ \text { HOMO } & \text { Highest Occupied Molecular Orbital } \\ \text { ICSD } & \text { Inorganic Crystal Structure Database } \\ \text { IEP } & \text { Isoelectric Point } \\ \text { IQY } & \text { Internal Quantum Yield } \\ \text { IR } & \text { Infrared } \\ \text { JCPDS } & \text { Joint Committee on Powder Diffraction Standards } \\ & \end{array}$




\begin{tabular}{ll} 
LED & Light Emitting Diode \\
LUMO & Lowest Unoccupied Molecular Orbital \\
MB & Methylene Blue \\
MCA & Melamine and Cyanuric Acid \\
MCA & Morphology Controlling Agent \\
MeOH & Methanol \\
MO & Methyl Orange \\
MS & Mass Spectroscopy \\
NADPH & Nicotinamide adenine dinucleotide phosphate \\
NHE & Normal Hydrogen Electrode \\
OER & Oxygen Evolution Rate \\
PEC & Photoelecrochemical \\
PSI & Photosystem 1 \\
PSII & Photosystem 2 \\
PTFE & Polytetrafluoroethylene \\
PVP & Polyvinylpyrrolidone \\
PXRD & Powder X-ray Diffraction \\
QY & Quantum Yield \\
RhB & Rhodamine B (organic dye) \\
RHE & Reversible Hydrogen Electrode \\
RT & Retention Time \\
SCE & Saturated Calomel Electrode \\
SD & Standard Deviation \\
SEM & Scanning Electron Microscopy \\
SSA & Specific Surface Area \\
STH\% & Solar-to-Hydrogen conversion efficiency \\
TAS & Transient Absorbance Spectroscopy \\
TCD & Thermal Conductivity Detector \\
TDDFT & Time Dependent Density Functional Theory \\
TEM & Transmission Electron Microscopy \\
TEOA & Triethanolamine \\
TGA & Thermo-Gravimetric Analysis \\
TOF & Turnover Frequency \\
TON & Turnover Number \\
UHV & Ultra-High Vacuum \\
UPS & Ultraviolet Photoelectron Spectroscopy \\
UV-Vis & Ultraviolet-Visible Spectroscopy \\
VB & Valence Band \\
VBE & Valence Band Edge \\
ZP & Z-ray Photoelectron Spectroscopy \\
\hline THetential
\end{tabular}


Roman symbols

\begin{tabular}{|c|c|}
\hline A & Absorbance (\%) \\
\hline$A$ & Surface area \\
\hline$a$ & Mass of the powdered adsorbant \\
\hline $\mathrm{c}$ & Speed of light $\left(\mathrm{ms}^{-1}\right)$ \\
\hline$c$ & BET constant \\
\hline $\mathrm{d}_{\mathrm{sc}}$ & Space charge layer depth \\
\hline E & Electric field \\
\hline $\mathrm{E}_{1}$ & Heat of adsorption of first layer \\
\hline $\mathrm{E}_{\mathrm{BG}}$ & Band gap (eV) \\
\hline$E_{\text {bulk }}$ & Total energy of atomic bulk per unit \\
\hline $\mathrm{E}_{\mathrm{CB}}$ & Bottom of conduction band \\
\hline $\mathrm{E}_{\mathrm{F}}$ & Fermi level \\
\hline $\mathrm{E}_{\gamma}$ & Energy of the incident x-ray \\
\hline $\mathrm{E}_{\mathrm{KE}}$ & Electron kinetic energy \\
\hline $\mathrm{E}_{\mathrm{L}}$ & Heat of adsorption for $n$ layers $>1$ \\
\hline $\mathrm{E}_{\mathrm{s}}$ & Incident irradiance \\
\hline$E_{\text {slab }}$ & Total energy of atomic slab \\
\hline $\mathrm{E}_{\mathrm{VB}}$ & Top of valence band \\
\hline $\mathrm{e}^{-}$ & Electron \\
\hline $\mathrm{F}$ & Faraday constant \\
\hline$\Delta \mathrm{G}^{0}$ & Gibbs free energy change $\left(\mathrm{kJ} \mathrm{mol}^{-1}\right)$ \\
\hline $\mathrm{h}$ & Planck's constant $(\mathrm{J} \cdot \mathrm{s})$ \\
\hline $\mathrm{h}^{+}$ & Hole \\
\hline $\mathrm{I}_{\lambda}$ & Incident spectral photon flux \\
\hline $\mathrm{J}_{\mathrm{BB}}$ & $\begin{array}{l}\text { Blackbody photon flux at wavelengths below that of } \\
\text { the band gap wavelength }\end{array}$ \\
\hline $\mathbf{J}_{s}$ & Absorbed photon flux \\
\hline$K$ & Scherrer shape factor \\
\hline $\mathrm{m}_{\mathrm{e}}^{*}$ & Electron mass \\
\hline $\mathrm{m}_{\mathrm{h}}^{*}$ & Hole mass \\
\hline $\mathrm{N}$ & Number of atomic units in slab \\
\hline$N_{A}$ & Avogadro's number \\
\hline $\mathrm{n}$ & Direct/Indirect band gap transition variable \\
\hline $\mathrm{n}$ & Refractive index \\
\hline
\end{tabular}




\begin{tabular}{|c|c|}
\hline $\mathrm{n}_{\mathrm{y}}$ & Real vibrational mode ' $y$ ' $(y \geq 1)$ \\
\hline $\mathrm{P}$ & Pressure $(\mathrm{Pa})$ \\
\hline$p$ & Partial vapour pressure \\
\hline$p_{0}$ & Saturation pressure of the physisorbed gas \\
\hline $\mathrm{R}$ & Reflectance (\%) \\
\hline$\Delta \mathrm{S}_{\text {mix }}$ & Entropy of mixing \\
\hline$s$ & Adsorption cross section of the powder \\
\hline $\mathrm{T}$ & Temperature (K) \\
\hline $\mathrm{T}$ & Transmission (\%) \\
\hline $\mathrm{U}_{\text {loss }}$ & Energy lost per photon \\
\hline$V$ & Molar volume of gas \\
\hline $\mathrm{V}_{\mathrm{FB}}$ & Flat band potential \\
\hline $\mathrm{V}_{\mathrm{ON}}$ & Onset potential \\
\hline $\mathrm{V}_{\mathrm{SCE}}$ & Voltage of Saturated Calomel Electrode \\
\hline $\mathrm{V}_{\mathrm{SSC}}$ & Voltage of Silver-Silver Chloride Electrode \\
\hline$v$ & $\begin{array}{l}\text { Particle velocity } \\
\text { Volume of gas adsorbed at standard temperature and }\end{array}$ \\
\hline$v_{a}$ & $\begin{array}{l}\text { pressure } \\
\text { Adsorbed gas quantity per volume to produce a } \\
\text { monolayer }\end{array}$ \\
\hline$v_{x}$ & Virtual state ' $\mathrm{x}$ ' $(\mathrm{x} \geq 1)$ \\
\hline
\end{tabular}

Greek symbols

$\begin{array}{ll}\alpha & \text { Absorption coefficient } \\ \beta & \text { Line broadening at half the maximum intensity } \\ \Delta \varphi_{\mathrm{sc}} & \text { Space charge layer potential } \\ \delta_{\mathrm{p}} & \text { Penetration depth } \\ \varepsilon_{0} & \text { Permittivity of free space } \\ \varepsilon_{\rho} & \text { Permittivity of the suspension medium } \\ \eta & \text { Viscosity of the suspension medium } \\ \eta_{\mathrm{c}} & \text { Photoconversion efficiency } \\ \theta & \text { Angle } \\ \lambda_{0} & \text { Wavelength of incident light in vacuum } \\ \lambda_{\mathrm{BG}} & \text { Band gap wavelength } \\ \mu & \text { Electrophoretic mobility } \\ \nu & \text { Frequency }\left(\mathrm{s}^{-1}\right)\end{array}$




$\begin{array}{ll}v_{\delta} & \text { Doppler shift frequency } \\ \sigma & \text { Standard Deviation } \\ \tau & \text { Mean size of the ordered crystalline domains } \\ \Phi & \text { Diameter }(\mathrm{mm}) \\ \Phi_{\text {loss }} & \text { Radiative quantum yield } \\ \Phi_{\text {loss P }} & \begin{array}{l}\text { Proportion of absorbed photons converted into conduction } \\ \text { band electrons }\end{array} \\ \phi & \text { Instrument work function }\end{array}$




\section{Introduction}

In response to noticeable climate change and growing energy demands, governments across the globe have pledged to drastically change the way they produce energy ${ }^{1}$. One of the most promising solutions to these two concerns is solar energy conversion. The 3,850,000 EJ of solar radiation which the Earth absorbs in one year, dwarfs our current energy consumption (around 474 EJ per year). Harnessing this power and using it efficiently would provide us with enough clean, renewable energy, to last us indefinitely.

Electrolysis of water is a conventionally employed and well-researched process. Hydrogen can be evolved from this reaction; however, it requires an enormous amount of energy. In order to bypass this energy input, but still maintain the production of hydrogen, the use of solar radiation is employed. Solar energy can be used to split water in three ways. Firstly, solar concentrators and catalysts are able to thermally decompose water, but this takes place at high temperatures, requiring materials to be stable at high temperature. More widely used is the second method, using photovoltaic devices to photoelectrolyse water. However currently, electrolysers and photovoltaic systems are reasonably costly ${ }^{2}$.

The third method is water photolysis; using specific wavelengths of radiation to promote charge carrier separation in a semiconductor; enabling water splitting through a photogenerated voltage. This option provides many advantages which the previous two do not. Many inorganic semiconductors are simply metal oxides/nitrides/sulphides; which are often non-toxic, cheap, and abundant. Furthermore, since the semiconductor photocatalyst houses both charge separation and dissociation reactions, losses are expected to be considerably $l_{0}{ }^{3}$. The fuel produced from the photochemical water splitting is termed "solar hydrogen" and can be used directly in, for example, fuel cells. Although a common view is that hydrogen is dangerous to transport due to its high flammability, it has been reported that moving hydrogen through pipelines proves more efficient than electricity ${ }^{4}$, which could prove to be of critical importance in the future. Another advantage of hydrogen fuel is that when used, in either direct combustion or a fuel cell stack, the resulting 'waste' is merely water - making the whole cycle essentially carbon free.

The semiconductor used in water photolysis will serve the same function as the light reaction centre in the natural photosynthetic reaction. However, 
the system's energy vector will not be ADP or NADPH, but $\mathrm{H}_{2}$ (solar hydrogen). As the water is oxidised, and oxygen is released, protons are reduced to hydrogen:

Equation 1. General reaction for water splitting

$$
\begin{gathered}
2 \mathrm{H}_{2} \mathrm{O}+4 h^{+} \rightarrow \mathrm{O}_{2}+4 \mathrm{H}^{+} \\
4 \mathrm{H}^{+}+4 e^{-} \rightarrow 2 \mathrm{H}_{2}
\end{gathered}
$$

"Solar hydrogen" can also be utilised as a chemical feedstock, to produce ammonia, in upgrading (hydrocracking) fossil fuels, producing methanol, and high purity acids amongst other chemicals. Solar hydrogen therefore could directly replace current feedstocks which are obtained via fossil fuels, reducing $\mathrm{CO}_{2}$ emissions ${ }^{5}$.

Historically, photoelectrochemical water splitting was first reported in 1972, on a $\mathrm{TiO}_{2}$ electrode under ultra-violet (UV) radiation. However, UV light represents merely $4 \%$ of solar radiation that hits the Earth's surface ${ }^{6}$. Considering this, and the fact that visible light constitutes over $44 \%$ of radiation incident on the Earth's surface, the development of visible lightdriven photocatalysts has become the major focus of researchers in the field $^{7,8,9}$. Regarding the performance, a generally accepted target of $10 \%$ "Solar-to-Hydrogen conversion efficiency" (STH\%) is required for commercial viability ${ }^{10}$. Yet this isn't the only requirement; a semiconductor photocatalyst must also be robust and stable enough to last for an extended period of time, and furthermore be cheap enough to be cost effective.

Since there are over 200 tested materials to date, and many different welldocumented approaches for fabricating each one, it is crucial to separate the types of compounds that are promising for further research, from the ones that are not. Pure water splitting on one photocatalyst is extremely difficult, both in terms of thermodynamics and charge carrier kinetics, therefore a route often pursued by academics is the investigation of half reactions. Half reactions can selectively produce usable fuels such as molecular $\mathrm{H}_{2}$ or $\mathrm{O}_{2}$, at the expense of an electron donor or acceptor, which itself is spent. Certain donors/acceptors can also be successively reduced and oxidised ('redox mediators'), and can be used in conjunction with two photocatalysts forming a Z-Scheme for overall water splitting, utilising two 
simultaneous half reactions that are spatially and temporally separated. Consequently extensive studies been carried out on compounds that can produce either hydrogen or oxygen separately in scavenger systems, such as $\mathrm{TiO}_{2}$ and $\mathrm{SrTiO}_{3}{ }^{11}$, which have relatively wide band gaps, and also into materials with narrow band gaps, such as $\mathrm{WO}_{3}{ }^{12}, \mathrm{GaAs}^{13}$ and $\mathrm{CdS}^{14}$. The typical trend in these reports is that wide band gap materials have low maximum photoconversion efficiencies, but have a high durability throughout a range of conditions. Narrow band gap semiconductors have the ability to absorb a wider portion of the electromagnetic (EM) spectrum, giving them higher theoretical maximum photoconversion efficiencies ${ }^{15}$. However, narrow band gap semiconductors often have band positions which inhibit the mutual evolution of oxygen and hydrogen, and can result in a photocorrosion effect, where redox reactions occur on the semiconductor itself instead of reducing/oxidising water ${ }^{16}$. Numerous compounds have been found to be active in the visible part of the spectrum; however, they have not provided a high enough efficiency at a low enough cost and the stability for commercialisation ${ }^{7,17,18}$. The search for newer visible light active photocatalysts, or further advancing the newer generation of photocatalysts, is now the current focus of the community.

In the research field, there are two main ways to study the activity of a photocatalyst. The first is to construct a photoelectrochemical (PEC) cell, which uses electrical bias to drive photoelectrons around a circuit, and thus generate a photocurrent. This is analogous to hydrogen production in the second method (colloidal reaction), which is to directly place the semiconductor photocatalyst in water, and monitor hydrogen and oxygen evolution using gas chromatography. A PEC cell relies on the efficiency of a photocatalyst, it also requires the refining of an extra component - the boundary between semiconductor and electrode/circuit (discussed later in section 2.1.1). Logically then, the cheapest and most facile way of screening different photocatalysts is in a colloidal setup, whereby the photocatalyst is immersed in water in a sealed reactor.

Therefore considering this strategy, the overall project goal was to utilise the simplicity of a colloidal set up in order to screen for highly efficient and novel visible light active photocatalysts, creating a materials platform for future water splitting systems to be built on. Furthermore, there were five main objectives which would enable the overall project aim to be achieved. The first was a thorough exploration and analysis into the design of semiconductor photocatalysts in the form of a comprehensive literature survey. It considered the robustness, cost, relative abundance of constituent 
elements, and finally the cleanliness of synthesis methods used in manufacturing photocatalysts.

The second was development of an efficient semiconductor photocatalyst for photocatalytic water oxidation using visible light. The third goal was to manufacture a highly active material for the production of molecular hydrogen from water under visible light irradiation. The fourth major aim in terms of experimental validation was to construct an overall water splitting system based on success and findings of the previous objectives. Ultimately, the synthesised compounds were studied using a variety of characterisation and analytical techniques in order to divulge a mechanistic insight into rate determining steps and factors which influence activity would be discovered. 


\section{Literature Survey}

\subsection{Fundamentals of semiconductor photoelectrochemistry}

\subsubsection{Semiconductor-electrolyte interface}

An equilibrium process takes place when a semiconductor is immersed in a redox electrolyte due to the disparate nature of the Fermi level; a flow of charge between the two phases ensues, forming a Helmholtz double layer ${ }^{19}$. The junction between the phases is akin to that of a Schottky barrier in a semiconductor-metal interface ${ }^{16}$. The Helmholtz double layer potential drop is accompanied by a further potential drop within the electrolyte at low ion concentrations in the Gouy layer. As the charge carrier density in a semiconductor is often rather small, and the Debye length is very large, a space charge region can be formed. This region dictates the electrode potential: the difference between semiconductor and electrolyte. The potential of the space charge layer $\left(\Delta \varphi_{s c}\right)$ is shifted downwards for a p-type semiconductor, and upwards for an n-type semiconductor (Figure 1), this is "band bending".

(a)

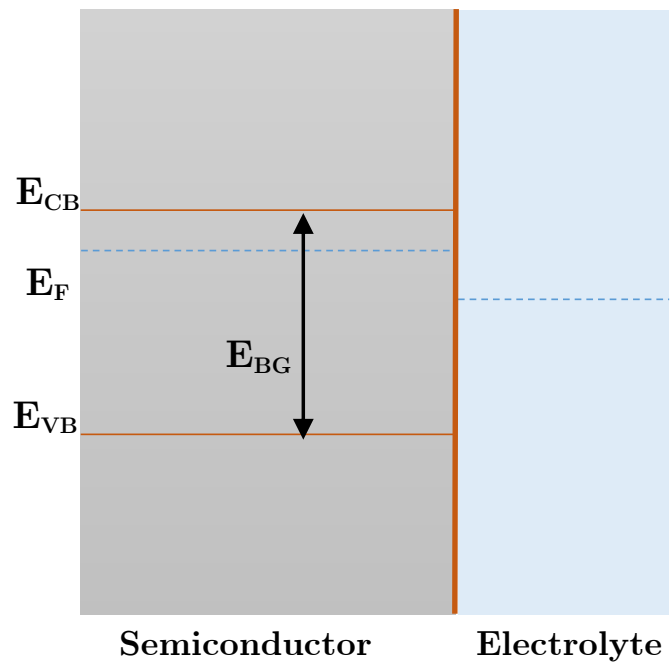

(b)

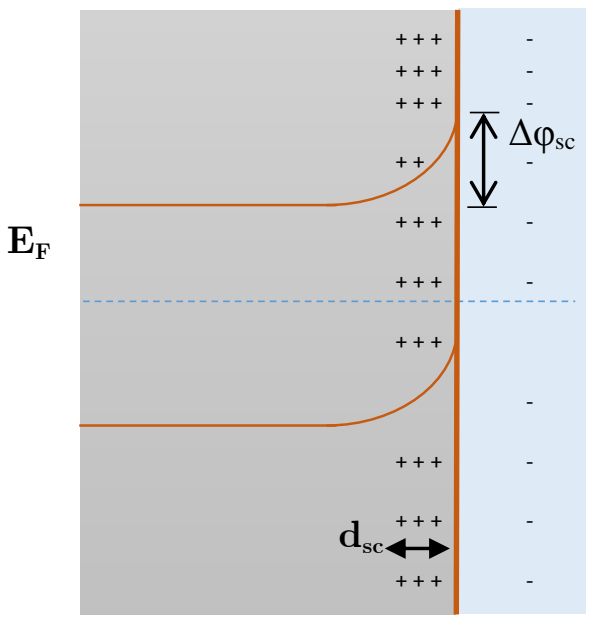

Figure 1. An example of an n-type semiconductor in contact with an electrolyte - (a) before equilibrium, (b) after equilibrium. Adapted from references ${ }^{15,20}$. Symbols are as follows: $\mathrm{E}_{\mathrm{CB}}-$ bottom of a conduction band, 
$E_{V B}-$ top of a valence band, $E_{B G}-$ band gap, $E_{F}-$ Fermi level, $d_{s c}-$ depth of space charge region.

For an n-type semiconductor, there are excess electrons in the bulk (more negative Fermi level), therefore in order to equilibrate, electrons flow from the surface of the semiconductor into the electrolyte, leaving a positive charge behind, the electrode potential.

The Fermi level in an intrinsic semiconductor should lie in the centre of the band gap (equal number of electrons and holes); doping with cations or anions shifts the Fermi level respectively to more positive potentials, and more negative potentials. As shown in Figure 1, the Fermi level in the semiconductor experiences a much larger shift than that of the one in the electrolyte solution. This is due to the lack of energy states in the semiconductor, excluding defect sites in the band gap region (in the forms of donor and acceptor states), in comparison to that of the electrolyte. A curious phenomenon which arises specifically in nanostructures is the diminished or complete absence of band bending. This occurs when then particle size is less than or equal to the depth of the space charge layer. This means the nanostructures can be unpredictable, demonstrating different behaviour depending on particle size and shape ${ }^{21}$. Electron-hole pairs induce electric fields which counteract band bending, and in turn raise the Fermi level towards the conduction band ${ }^{22}$.

\subsubsection{Charge carrier generation}

Electrons located in the valence band (VB) of a semiconductor can be excited by photons which have energy greater than the band gap of semiconductor - the energy difference between the top of the filled valence band and the bottom of the empty conduction band (CB). The photoexcited electrons are promoted from the top of the VB to the bottom of the CB, simultaneously leaving positively charged holes in the VB (Figure 2). If the electron-hole pairs (exciton) escape recombination they can travel to the surface of semiconductor and act as redox species for the splitting of water, organic decomposition, or the reduction of $\mathrm{CO}_{2}{ }^{23}$. 




Figure 2. Idealised diagram of charge separation in a semiconductor photocatalyst.

In order to reduce protons to hydrogen fuel, electrons in the $\mathrm{CB}$ are required to have a reduction potential that is more negative than the redox potential of $\mathrm{H}^{+} / \mathrm{H}_{2}, 0 \mathrm{eV}$ vs. Normal Hydrogen Electrode (NHE). Water oxidation occurs when the hole potential is more positive than the redox potential of $\mathrm{O}_{2} / \mathrm{H}_{2} \mathrm{O},+1.23 \mathrm{eV}$ vs. NHE. Therefore, a minimum theoretical band gap of $1.23 \mathrm{eV}$ is required.

This corresponds with the Gibbs free energy change $\left(\Delta \mathbf{G}^{0}\right)$ for overall water dissociation:

Equation 2.

$$
\mathrm{H}_{2} \mathrm{O}+2 \mathrm{e}^{-} \rightarrow \mathrm{H}_{2}+0.5 \mathrm{O}_{2}=237 \mathrm{~kJ} \mathrm{~mol}^{-1}
$$

Equation 3.

$$
\Delta G^{\circ}=-n F E^{\circ}
$$

Where $\mathrm{n}$ is the number of electrons per mole product ( 2 electrons in this instance), $\mathrm{F}$ is the Faraday constant (units: $\mathrm{C} \mathrm{mol}^{-1}$ ) and $\mathrm{E}^{0}$ is the electrode potential;

$$
\begin{gathered}
\Delta G^{\circ}=-2 \cdot\left(9.64 \times 10^{4}\right) \cdot 1.23 \\
\Delta G^{\circ}=2.46 \mathrm{eV} \times\left(9.64 \times 10^{4}\right) \\
\Delta G^{\circ}=237 \mathrm{~kJ} \mathrm{~mol}^{-1}
\end{gathered}
$$


In essence the minimum practical energy required to drive a photocatalytic reaction is much higher due to energy losses associated with the overpotentials required for the two chemical reactions and the driving force for charge carrier transportation. Studies disagree on this minimum limit, but according to reports it can be as low as $1.6 \mathrm{eV}^{24}$ and as high as $2.5 \mathrm{eV}^{25}$. Therefore the reaction in itself is thermodynamically difficult. Since ultraviolet (UV) light $(\leq 400 \mathrm{~nm})$ constitutes less than $4 \%$ of the solar spectrum, and $43 \%$ of light is in the visible region $(400-750 \mathrm{~nm})$, development of a photocatalyst with activity from the UV through to visible wavelengths $(400-750 \mathrm{~nm})$ and with a VB and CB that straddle the reaction potentials is one of key goals in obtaining optimum solar energy to fuel efficiency ${ }^{26}$. The band gaps of some simple semiconductors have been measured by various authors and are shown below (Figure 3). As can be seen from Figure 3, the general trend is that oxide semiconductors have very deep (positive vs. NHE) valence bands, facilitating stable oxidation reactions, but limiting light absorption. Conversely non-oxide semiconductors having smaller band gaps and more negative band positions, yet can suffer from photocorrosion.

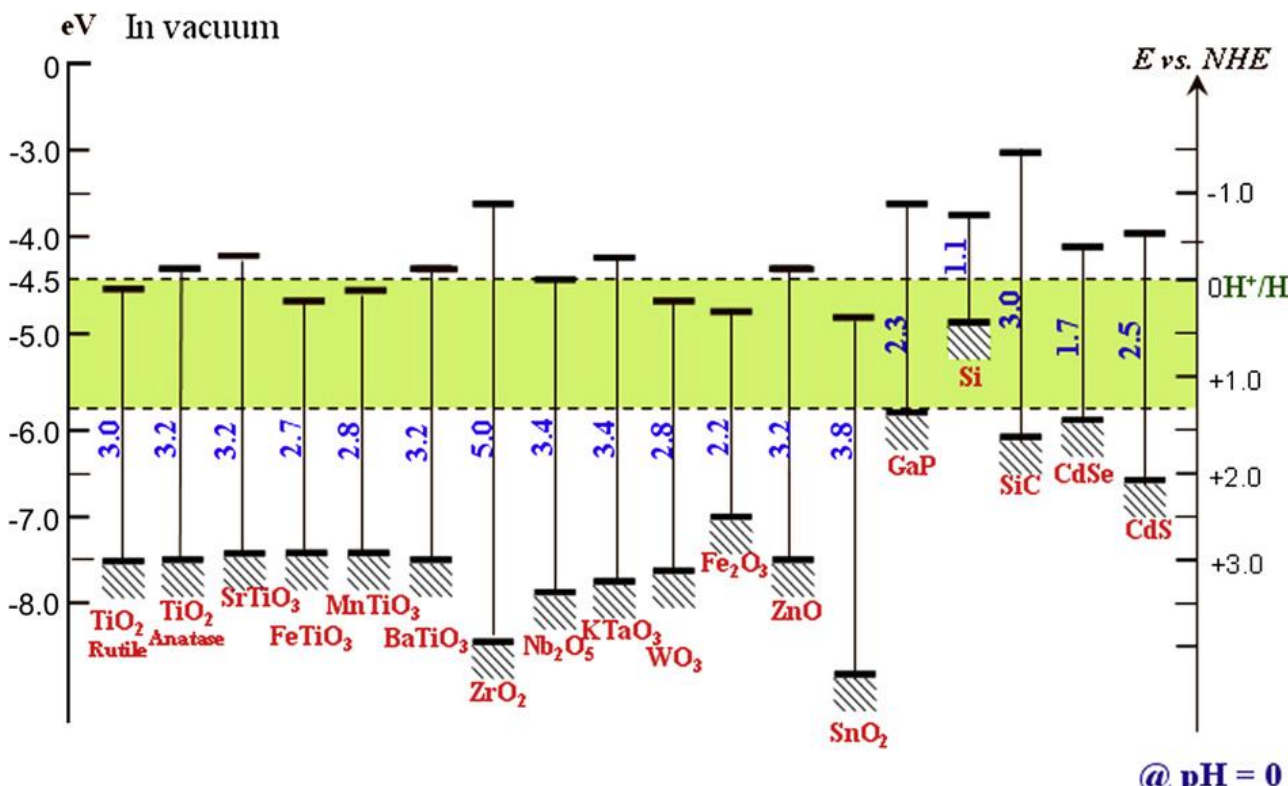

Figure 3. Positions (black) and magnitudes (blue) of the most well researched semiconductors (red). The green region represents the redox minimum between 0 and $1.23 \mathrm{~V}$ vs NHE. Reproduced from reference ${ }^{26}$ 


\subsubsection{Photoelectrochemistry}

The first demonstration of conventional water splitting as we know it used a titania anode and a platinum cathode, and the reaction was driven by an electrical bias ${ }^{6}$, therefore the system was in fact a photoelectrochemical cell. In modern photoelectrochemical cells (PEC cells), there are three electrodes, immersed in a redox electrolyte. The semiconductor photocatalyst acts as the working electrode, and the counter electrode is often platinum gauze, which due to the tiny overpotential is perfect for reducing protons to hydrogen fuel. The modern reference electrode is either $\mathrm{Ag} / \mathrm{AgCl}$ (Silver-Silver Chloride - denoted SSC) or saturated calomel (Saturated Calomel Electrode - SCE), but historically, a normal or reversible hydrogen electrode (NHE/RHE) was used. They have been replaced due to their lack of practicality. However, they are often referenced and converted accordingly: $\mathrm{V}_{\mathrm{SSC}}=+0.197 \mathrm{~V}$ versus $\mathrm{NHE}, \mathrm{V}_{\mathrm{SCE}}$ $=+0.242 \mathrm{~V}$ versus $\mathrm{NHE}{ }^{27}$.

When an external bias is applied to the system, it is necessary to measure the current produced from the counter to the working electrode. The reference electrode should draw no current at all. This applied bias has the role of changing the Fermi levels of both the semiconductor and solution, often resulting in two different Fermi levels ${ }^{15,27,28}$. When there is no external bias applied to the system, and no band bending, the potential of the Fermi level is termed the flatband potential $\left(\mathrm{V}_{\mathrm{FB}}\right)$. Here there is no photocurrent since all charge carriers recombine. If the flatband potential is high enough, it can be approximated to be the onset potential $\left(\mathrm{V}_{\mathrm{ON}}\right)$ for the photocurrent $^{22}$. Both flatband potentials of the CB and VB are determined intrinsically by the material, but are affected slightly by the $\mathrm{pH}$ of the electrolyte $^{28}$.

One of the main reasons behind performing photoelectrochemistry is not only to study and monitor the photocurrent, but to also drive the reduction of protons and produce hydrogen fuel. If the semiconductor's conduction band edge is fractionally anodic in comparison with the $\mathbf{H}_{2}$ redox potential, then a voltage must be applied externally to the system in order to reduce the photogenerated electrons and turn $\mathrm{H}^{+}$into $\mathrm{H}_{2}$ (Figure 4, below). 


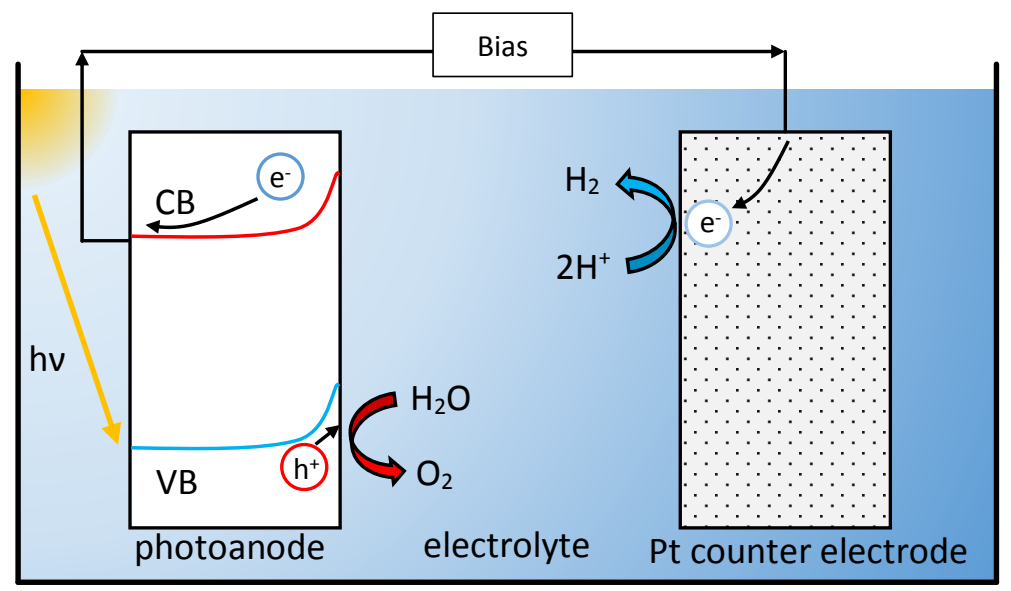

Figure 4. A typical PEC cell for photoelectrochemistry, with applied bias to drive proton reduction. Inspired by Kudo and Miseki ${ }^{23}$.

Using photoelectrochemistry, it is possible to split up the overall reaction for water splitting into different parts, which are shown below ${ }^{29}$.

Equation 1. General reaction for water splitting

$$
\mathrm{H}_{2} \mathrm{O} \rightarrow \mathrm{H}_{2}+0.5 \mathrm{O}_{2}
$$

In basic solution:

Equation 4. Basic anodic reaction

$$
2 h^{+}+2 \mathrm{OH}^{-} \rightleftarrows 0.5 \mathrm{O}_{2}+\mathrm{H}_{2} \mathrm{O}
$$

Equation 5. Basic cathodic reaction

$$
2 e^{-}+2 \mathrm{H}_{2} \mathrm{O} \rightleftharpoons \mathrm{H}_{2}+2 \mathrm{OH}^{-}
$$

In acidic solution:

Equation 6. Acidic anodic reaction

$$
2 h^{+}+\mathrm{H}_{2} \mathrm{O} \rightleftharpoons 0.5 \mathrm{O}_{2}+2 \mathrm{H}^{+}
$$

Equation 7. Acidic cathodic reaction 


$$
2 e^{-}+2 H^{+} \rightleftharpoons H_{2}
$$

Note: these equations are for an n-type semiconductor, where the semiconductor is the anode. If the semiconductor is p-type, then it acts as the cathode.

Another side note on photoelectrochemical reactions has been noted by Rajeshwar regarding specific terminology ${ }^{25}$. The author mentions that the term "photoelectrochemical" should describe any reaction where light is used to enhance the electrochemical process. This thermodynamic process could be either "uphill" (positive $\Delta \mathrm{G}^{0}$ - "photosynthetic") or "downhill" (negative $\Delta G^{0}$ - "photocatalytic"). The term "photoelectrolysis" can be used for a photoelectrode in an electrochemical cell. "Photocatalysis" should be applied for those systems which are suspensions or colloidal, and require no external bias (but still with a positive $\Delta \mathrm{G}$ ). The phrase "photoassisted water splitting" should be used to describe experimental setups where the reaction process is a product of both photon energy and that supplied by bias.

One of the main problems with a photoelectrochemical setup is that the connection between the semiconductor photoanode and the driving bias must be almost perfect in order to gain a true insight into the activity of the photocatalyst. For example, the semiconductor could be theoretically efficient, but if the connection is poor, electrons cannot be shuttled to the counter electrode, and recombine with holes. Therefore the complexity of the device adds to the difficulty and hence cost of making a solar-to-fuel system, which is a major problem in the field. Often, a thin semiconductor film is grown onto a conducting substrate, with the aim that the penetration depth of light does not exceed the thickness of the film. For wavelengths 200 to $1000 \mathrm{~nm}$, the penetration depth in most semiconductors $\left(\delta_{\mathrm{p}}\right.$, the distance at which the intensity of incident electromagnetic radiation falls to 1 /e or about $\sim 37 \%$ of its original value in a given material) increases with larger wavelengths, a direct consequence of band-gap absorption. That is to say most blue light is absorbed at the surface, whilst red light penetrates deep into the bulk. This becomes crucial in semiconductors which are used as photocatalysts because photogenerated charge carriers must migrate to the surface in order to participate in redox reactions. Therefore in thin films, excitons created deep in the bulk (in photocatalysts responding to visible light) are less likely to generate a photocurrent and split water. Along with poor film adhesion to the conductive substrate, it often leads to poor photocurrent in devices ${ }^{30}$. 


\subsubsection{Photocatalytic water splitting}

The previous section focused on using a semiconductor simply as a working electrode, and having the proton-electron recombination centre - the counter electrode, as a separate entity. There are many studies and reviews however which focus on colloidal systems; suspensions of photocatalyst particles within a glass batch reactor immersed in an electrolyte ${ }^{7,31,32}$. No external bias is applied, so the particles act naturally as a functioning anode and cathode (Figure 5). This is often a good test of a semiconductors' photocatalytic ability; one can see if hydrogen and oxygen can be evolved without bias, which is the eventual goal of idealised water splitting. It is arguably thought that powdered/colloidal catalyst systems would be ideal for large scale industrial hydrogen generation purely because of its straightforward nature ${ }^{23}$. There are no electrical connections needed, and if a good scavenger is used, an accurate efficiency can be obtained. However, some studies argue that colloidal photocatalyst systems have more use as a simple and effective method for testing the efficiency of different compounds. 


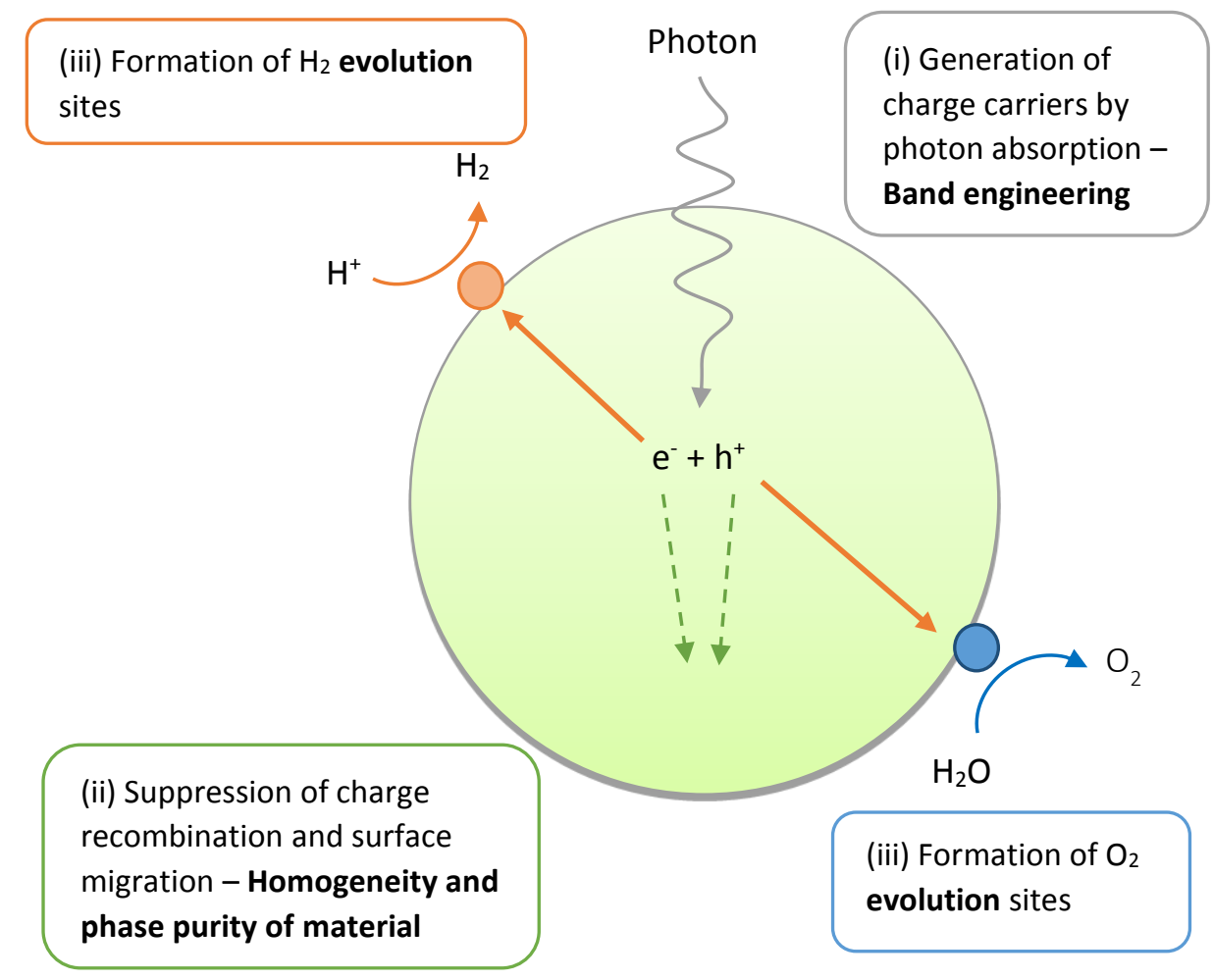

Figure 5. An illustration of the three main processes in photocatalytic water splitting. Adapted from reference ${ }^{23}$

Having hydrogen and oxygen together in a contained atmosphere is potentially explosive. Due to this fact, safety protocols should be followed when using a suspension system and an efficient photocatalyst for pure water splitting (e.g. using inert atmospheres and small volume reactors). Water splitting using colloidal systems on a larger industrial scale however might be impractical, as flammability limits might be reached unless the system is made more complex by using compressors and separating units to effectively shuttle hydrogen and oxygen mixtures away from reactors. However for lab-scale materials testing, suspension systems are an effective tool in particular for a half reactions. For instance, if a compound is found to be highly efficient, it could then be integrated into a PEC cell system, and hydrogen and oxygen would be produced in separate compartments of one cell.

Electron acceptors and donors (vernacular; electron and hole 'scavengers', respectively) are compounds which have a very high relative electronegativity (for electron acceptors/scavengers) or electropositivity 
(for electron donors/hole scavengers), and can therefore stop the production of either hydrogen (electron donor) or oxygen (electron acceptor) by combining with the respective charge carrier ${ }^{33}$. Scavenger compounds must also have a high solubility in water in order to donate or accept charge readily. Electron acceptors include $\mathrm{S}_{2} \mathrm{O}_{8}{ }^{2-}, \mathrm{Ce}\left(\mathrm{SO}_{4}\right)_{2}, \mathrm{FeCl}_{3}$ but the most frequently used is $\mathrm{Ag}^{+}$from $\mathrm{AgNO}_{3}$, as it has been shown to scavenge electrons more efficiently than any other electron acceptor ${ }^{34,35}$. Electron donors commonly used are: ethanol, methanol, triethanolamine (TEOA), $\mathrm{Na}_{2} \mathrm{~S}, \mathrm{Na}_{2} \mathrm{~S}_{2} \mathrm{O}_{3}$ and $\mathrm{Na}_{2} \mathrm{SO}_{3}$.

The overall water splitting reaction process can be considered to contain three main parts (as shown in Figure 5): (i) charge carrier (electron/hole) promotion following absorption of a photon, (ii) charge carrier separation and migration, (iii) redox reaction between surface species and the charge carriers. The first two steps are interrelated, and are both photophysical processes, whilst the final step is a purely chemical process. A total photocatalytic reaction is hence a complex combination of photophysical and photochemical processes. Besides research being carried out regarding enhancing the ability of visible light harvesting in a semiconductor (part (i)), there is also considerable development in the water splitting field with view to develop a rapid charge separation system (part (ii)). It has also standard practice to experiment with the material's particle morphology and alter the surface structure modification to increase the reaction rate of redox couples (part (iii)).

There have been many examples within semiconductor literature (which will be mentioned later in this chapter) that are able to selectively produce hydrogen or oxygen from water using charge carrier scavengers. Unfortunately, most are unable to evolve both gases in their correct stoichiometric ratio due to electron/hole recombination and lack of active sites with low overpotential on the surface. This leads to the obvious conclusion that although the correct positioning and energy of the band gap are important criteria, there are other factors which govern photocatalytic activity. Even if the hole and electron potential are sufficient to thermodynamically split water, they will recombine if active sites at the surface are not present. 'Cocatalysts', as they are termed, are usually loaded on the surface of the semiconductor to act as active sites for pure water splitting $(\mathrm{NiO}), \mathrm{H}_{2}$ evolution $(\mathrm{Pt}, \mathrm{Au}, \mathrm{Ru})$ or oxygen evolution $\left(\mathrm{RuO}_{2}\right)$. This is because many oxide based semiconductors do not possess a conduction band which is high enough to reduce protons to $\mathrm{H}_{2}$ without catalytic help. Most crucially, cocatalysts also act as electron or hole sinks, 
and prevent the recombination of charge. By localising electron and holes at specific catalytic sites for a much longer time period, the probability of a charge carrier participating in a redox reaction greatly increases. One report even suggests the double loading of cocatalysts; which improves the performance even further ${ }^{9}$. The in situ photodeposition of cocatalysts was demonstrated by Bamwenda, and shown to be the most effective method of loading a cocatalyst - in terms of activity ${ }^{36}$. This is due to the catalyst being deposited directly and selectively on an active site, where it is most effective. Without cocatalyst loading, factors such as charge carrier recombination - fuelled by kinetic competition, may occur at a superior rate than the necessary surface redox reactions, which can be detrimental to the reactivity in an overall water splitting reaction. However, there are exceptions; metal oxides for the most part do not need an oxidation catalyst due to the correct positioning and composition of oxygen $2 p$ orbitals. With the exception of a few photocatalysts ( $\mathrm{ZnO}$ for example), oxides are resistant to photocorrosion.

\subsubsection{Efficiency calculations}

Many methods and equations can be used to calculate the efficiency of a semiconductors ability to decompose water. However, several excellent review papers have been published, ${ }^{15,23,27}$ with proposed guidelines to conform to when performing efficiency analysis. In this section, the most important equations from these papers will be discussed, and it will be divulged which of these are suitable for analysis.

Commonly, the production of hydrogen or oxygen is given with the unit micromole of product per hour, per gram of photocatalyst; ' $\mu \mathrm{mol} \mathrm{h}^{-1} \mathrm{~g}^{-1}$ '. Despite being acceptable unit for presenting rates, it is unsuitable for determining efficiency, since most gas evolution rates depend on the experimental conditions: light source, reactor size, amount of catalyst, and type of Gas Chromatograph (GC) amongst others. This makes evolution rates incomparable to some extent. The number of incident photons can be calculated using a thermopile or silicon detector/photodiode. Unfortunately, the number of absorbed photons (by the photocatalyst) is often considerably less than that of incident light due to scattering effects and transmission. But, the amount of photocatalyst used in an experiment should still be thought about carefully; whereby the catalyst concentration is adjusted to account for variable dispersivity, and minimise reflective losses. Therefore, it is possible to use a trial and error process, whereby 
catalyst concentration is varied, and then compared to the product produced in ' $\mu \mathrm{mol} \mathbf{h}^{-1} \mathrm{~g}^{-1}$ '. As demonstrated by Li Puma, it is also possible to model the ideal catalyst concentration in a reactor by optimising the optical thickness of a semiconductor ${ }^{37}$. However, to simplify matters in this study, all scattered (externally reflected) photons by semiconductor particles and reactor walls, are not taken into account herein. The quantum yield obtained should actually be termed 'Apparent Quantum Yield' (AQY) where incident photon flux is measured outside the reactor. A more accurate measurement of the number of photons incident on the photocatalyst surface can be performed by subtracting the flux from behind the reactor, from the flux measured in front of the reactor. The quantum yield in that case is termed 'internal quantum yield' (IQY). The number of reacted electrons can be calculated by using the amount of evolved hydrogen or oxygen ${ }^{38}$. This can be understood as the percentage of photons which cause charge carriers to become reactive.

Equation 8. Internal Quantum yield

$$
I Q Y(\%)=\frac{\text { Number of reacted electrons }}{\text { Number of } \text { incident photons }} \times 100
$$

The Turn-Over Number (TON, Equation 9) is used to illustrate whether a process is truly catalytic or not. Successfully used in homogenous catalysis, the TON can also be applied to heterogeneous catalysis with some minor modifications. It is normally defined as the number of reacted molecules per active site, but the active site does depend on the type of reaction (reduction/oxidation) $^{38}$. Sometimes the number of active sites can be very roughly approximated to the number of molecules in the photocatalyst, though this grossly underestimates the actual turnover number.

Equation 9. Turnover Number

$$
\text { TON }=\frac{\text { Number of reacted electrons }}{\text { Number of active sites on photocatalyst }}
$$

QY and TON are different than Solar-To-Hydrogen conversion efficiency, STH\%, which is defined as:

Equation 10. STH\% 


$$
S T H(\%)=\frac{\text { Output energy as } \mathrm{H}_{2}}{\text { Energy of incident solar light }} \times 100
$$

A worthy note is that although the STH\% equation only takes into account 'output energy as $\mathbf{H}_{2}$ ', it cannot be used for half-reactions $\left(\mathrm{H}_{2}\right.$ production with a hole scavenger), and must be only applied to overall water splitting reactions, such as a $\mathrm{Z}$-scheme. This is because the scavenger is consumed, and the process does not solely rely on water. That is to say that in the reformation of methanol (as an example hole scavenger), hydrogen is produced not only from water but from methanol itself. Redox mediators in Z-scheme reactions (such as $\mathrm{Fe}^{2+/ 3+}$ and $\mathrm{I}^{-} / \mathrm{IO}_{3^{-}}$) aren't exhausted/consumed, and therefore $\mathrm{Z}$-scheme systems can be evaluated using STH\%.

Besides efficiency calculations, it is often noteworthy to examine the data using basic stoichiometry. In an overall water splitting reaction, hydrogen and oxygen should evolve in an approximate 2:1 ratio in the absence of an electron donor/acceptor. If the amounts are not in a stoichiometric ratio, then it is clear that the reaction isn't proceeding photocatalytically; other factors could be coming into play (leaking, degradation etc). During oxygen evolution, $\mathrm{Ag}^{+}$is reduced to $\mathrm{Ag}^{0}$ on the surface of some particles (from electron scavenger $\mathrm{AgNO}_{3}$ ). This leads to the deactivation of the photocatalyst as Ag metallic species block light absorption.

\subsubsection{Thermodynamic limits}

In section 2.1.2, it was mentioned that due to energy losses associated with overpotentials, the theoretical minimum band gap of a semiconductor required to split water was not $1.23 \mathrm{eV}$, but much higher. Murphy et al. investigated the overpotentials which cause the band gap to be increased; their most important findings are presented in this section ${ }^{15}$. Murphy et al. also note that their findings are in agreement with other groups; Bolton et al. and Weber \& Dignam ${ }^{39,40,41}$.

In an ideal semiconductor, all photons with energy greater than $\mathrm{E}_{\mathrm{BG}}$ would be absorbed. Valence band electrons can earn promotion to the conduction band, and then they are transferred to the electrolyte, where a pair of such 
electrons can lead to a hydrogen molecule being produced. The absorbed photon flux would be:

Equation 11. Absorbed photon flux $\left(\mathrm{J}_{\mathrm{s}}\right)$

$$
J_{s}=\int_{0}^{\lambda_{B G}} I_{\lambda}(\lambda) d \lambda
$$

where $\lambda_{\mathrm{BG}}$ is the band gap wavelength $\left(\lambda_{\mathrm{BG}}=\mathrm{hc} / \mathrm{E}_{\mathrm{BG}}\right)$ and $\mathrm{I}_{\lambda}$ is the incident spectral photon flux $\left(\mathrm{m}^{-2} \mathrm{~nm}^{-1} \mathrm{~s}^{-1}\right)$. One can then form an upper limit to the photoconversion efficiency:

Equation 12. Limits of photoconversion efficiency $\left(\eta_{c}\right)$

$\eta_{C}=\left\{\begin{array}{cl}\frac{J_{s} \Delta G^{\circ}\left(1-\Phi_{\text {loss })}\right.}{E_{S}} & \text { if } E_{B G} \geq \Delta G^{o}+U_{\text {loss }} \\ 0 & \text { if } E_{B G} \leq \Delta G^{o}+U_{\text {loss }}\end{array}\right.$

The term ' $\Phi_{\text {loss' }}$ ' accounts for the radiative quantum yield, which is more specifically the ratio of re-radiated photons to absorbed photons. Therefore, the collective term ' $1-\Phi_{\text {loss' }}$ ' is the amount of absorbed photons which have led to conduction band electrons being produced. The re-radiation of photons is due to blackbody radiation from the excited state. The value of $\Phi_{\text {loss P }}$ that relates a maximum value of the efficiency is given by:

Equation 13. Proportion of absorbed photons converted into conduction band electrons $\left(\Phi_{\text {loss } \mathrm{P}}\right)$ :

$$
\Phi_{\text {loss } P} \approx 1 / \ln \left(J_{s} / J_{B B}\right)
$$

where $J_{\mathrm{BB}}$ is the blackbody photon flux at wavelengths below that of the band gap wavelength, $\lambda_{\mathrm{BG}}$. $\Phi_{\text {loss } \mathrm{P}}$ has been calculated to be small, less than $0.02 \mathrm{eV}$ for the range of band gap wavelengths that photocatalysts lie in, and for all intents and purposes, is independent of the spectrum of incident radiation.

In Equation 12, the term $U_{\text {loss }}$ is the energy lost per photon; which turns out to be a significant loss term, unlike $\Phi_{\text {loss }}$ P. This enables a criterion to be set up for the lower limit of the band gap energy of a semiconductor that can be used for water splitting. The loss is composed of two main parts; a thermodynamic part and a kinetic part. The thermodynamic part 
is equal to $T \Delta S_{\text {mix }}$, where $T$ is temperature and $\Delta S_{\text {mix }}$ is the entropy of mixing which governs the production of conduction band electrons by photon absorption $(\geq 0.4 \mathrm{eV})$. Combined with the second contribution, which arises from kinetic losses due to the overpotentials for oxygen and hydrogen ( $\geq$ $0.4 \mathrm{eV}$ ), the estimation for $\mathrm{U}_{\text {loss }}$ is $\geq 0.8 \mathrm{eV}$. This therefore leaves the minimum band gap energy at $2.03 \mathrm{eV}$, which means that the limiting band gap wavelength is $610 \mathrm{~nm}$ (as shown in Figure 6).

Murphy et al. reviewed the effect on photoconversion efficiencies caused by the different light sources ${ }^{15}$; as one can see from Figure 6, using different light sources yields different results. The conclusion drawn is that the spectra of artificial light sources, such as Xenon, mercury, and xenonmercury lamps, do not accurately represent the spectrum of solar irradiation. This leads to quoted efficiencies being higher than those thermodynamically possible for photocatalysts. Despite this, arc lamps are the most accurate representation of the solar spectrum available for most scientific research groups.

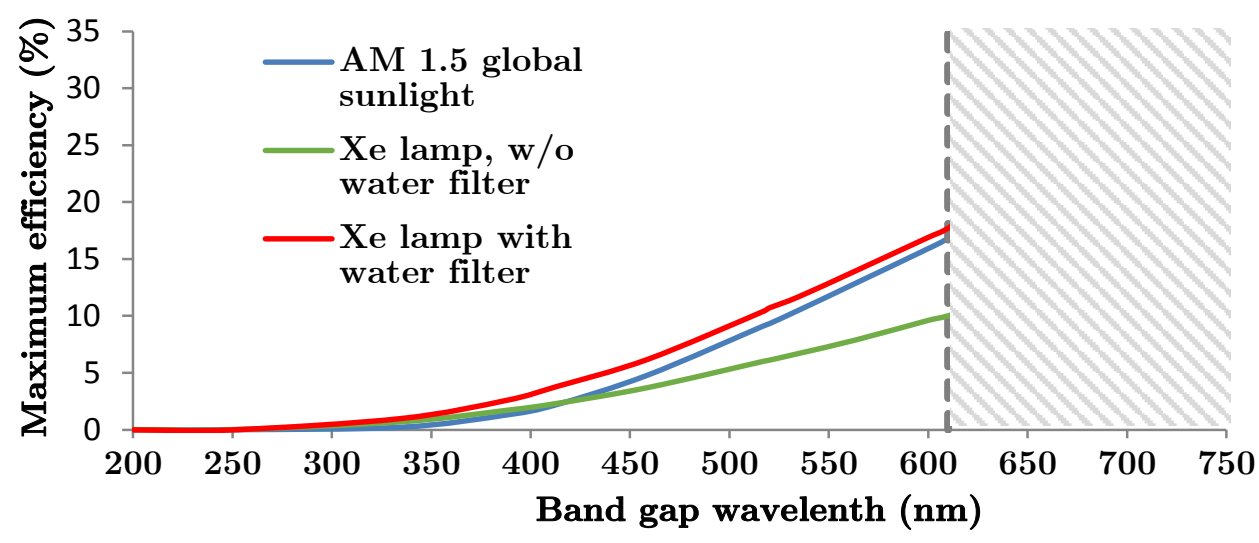

Figure 6. The maximum photoconversion efficiency possible as a function of the band gap wavelength ( $150 \mathrm{~W} \mathrm{Xe})$. Indicated with a dashed line is the maximum band gap wavelength at $610 \mathrm{~nm}$. This corresponds with a minimum band gap of $2.03 \mathrm{eV}$ for water splitting. Reproduced from reference $^{15}$.

The air mass spectra (AM) are the most accurate way of replicating the spectrum of solar radiation found on the Earth's surface ${ }^{15}$. The AM spectra account for the amount of the Earth's atmosphere radiation has to go 
through, and also, what radiation is filtered out in the process. An AM 1.5 filter removes some UV and IR radiation, like our own atmosphere, and also takes into account the angle at which the sun's radiation passes through. AM 1.5 corresponds to $48.2^{\circ}$, derived from $\mathrm{AM}=1 / \cos \alpha(\alpha$ is the zenith angle of the sun), relative to $\alpha=0$, with the illuminated surface inclined at $37^{\circ}$, leaving an $11.2^{\circ}$ incidence angle. The two types of AM spectra are direct and global. Direct is just from a single $\alpha$ angle, while global takes into account scattered light from all angles.

Murphy et al. concluded that at short wavelengths a xenon lamp with no water filter has a larger spectral flux in comparison to the AM 1.5 global spectrum (in the real world the atmosphere absorbs most harmful UV radiation at $\lambda \leq 300 \mathrm{~nm}$ ). Furthermore, older xenon lamps have decreased UV flux in comparison with newer ones. One last point, which can be seen from Figure 6 is that the xenon lamp without a water filter can yield overestimated efficiencies at $\lambda \leq 410 \mathrm{~nm}$; this means that wide band gap materials are often overestimated, and narrow band gap materials are underestimated.

It is often worthwhile to also conduct experiments with, and without a long pass filter, especially for visible light activated photocatalysts. Whilst no filter illuminates the sample with both UV and visible light, long pass filters block out all UV light ( $\lambda \geq 400$ or $420 \mathrm{~nm}$ ). Therefore a comparison can be drawn between the contribution of UV or visible photons on the photocatalytic activity of a compound. This is especially helpful with new or doped visible light photocatalysts, where one might want to see if the main contribution comes from UV or visible photons. A classic example of this is nitrogen doped $\mathrm{TiO}_{2}$; the absorption spectra shows an absorption tail into the visible range, however, the compound is unable to evolve molecular oxygen under visible light, even in the presence of scavengers ${ }^{42}$. Therefore the contribution of visible photons to water splitting activity is almost zero, although the compound can degrade some organic molecules.

\subsection{Characterisation methods for photocatalysts}

When engineering either new compounds, or attempting to modify existing photocatalysts, it is extremely important to classify them correctly. A wide variety of characterisation methods are available at present, and these not only help to confirm results, but also aid in understanding the inner workings of a photocatalyst. 


\subsubsection{UV-Visible spectroscopy}

Calculating the absorption spectra for a material is invaluable, it enables analysis of the wavelength range where a material absorbs, and also how strong. UV-Visible spectrophotometers ('UV-Vis') probe through the electromagnetic spectrum from ultraviolet through to the visible range. Molecules often undergo electron transitions at these frequencies, since semiconductors have discrete energy levels corresponding to allowed electron states. Therefore one can determine the size and type of band gap (direct or indirect). This is done using a Tauc plot, often used in thin films ${ }^{43}$ :

Equation 14. Tauc relation

$$
(\alpha h v)=A\left(h v-E_{B G}\right)^{n}
$$

Where $\alpha$ is the absorption coefficient, $h v$ is the energy of the incident radiation, $\mathrm{A}$ is the constant which is based on effective masses of electrons and holes, and $\mathrm{n}$ can take the values of 0.5 and 2 , for a direct or indirect band gap transition.

Often, UV-Vis spectrophotometers will give data where the wavelength is on the abscissa and either the absorbance $(A)$ or reflectance $(R)$ on the ordinate. The absorption coefficient can be calculated using:

Equation 15. Kubelka-Mulk relation

$$
\alpha=\frac{(1-R)^{2}}{2 R}
$$

The reflectance is related to the absorption by the following equations:

Equation 16. Transmission-reflectance-absorption relation

$$
1=T+R+A
$$

where $\mathrm{T}$ is the transmission in $\%$.

Often spectrophotometers will give the absorbance as a function of a logarithm. If this is to be converted to a percentage then the following relation can be used: 
Equation 17. Absorbance conversion

$$
A(\%)=1-10^{-A_{\log 10}}
$$

The band gap of semiconductor is approximately equal to the absorption edge, and so one can also find this from extrapolating towards the $\mathrm{x}$-axis on a plot of wavelength (x-axis) versus absorption $\left(\log _{10} \mathrm{y}\right.$-axis), similar to a Tauc plot (Equation 14). Then using the following relation, calculate the band gap:

Equation 18. Planck relation (nm)

$$
E=\frac{h c}{\lambda} \rightarrow h v=\frac{1240}{\lambda}
$$

The absorption edge can be blue shifted by decreasing the particle size, and is often attributed to either a quantum size effect or a direct charge-transfer transition. If the band gap region possess a long tail, and appears to be red shifted, then this would conclude that there are additional sub band gap states present ${ }^{44}$.

\subsubsection{Gas Chromatography}

The process of water splitting often yields two gases - oxygen and hydrogen, and being able to calculate the amount of each produced is vital. Gas chromatography is used to analyse concentrations and amounts of gas in a given atmosphere, often to a great resolution. A sample of gas is injected; the elements of the gas (mobile phase) are separated by a stationary phase 'column' (often stainless steel tubing, densely packed with diatomaceous earth), compounds elute at different times and pass through the detector where a signal is generated and data can be produced.

Gas Chromatographs (GC's) are usually fitted with either a Thermal Conductivity Detector (TCD), or a Flame Ionisation Detector (FID). The most suitable detector for simultaneous hydrogen and oxygen detection would be a TCD. As the name suggests, the TCD detects changes in thermal conductivity produced by the sample from a standard gas flow (carrier gas), originally using a wheatstone bridge and resistors which present varying heat loss depending on analyte. Standard carrier gases are nitrogen, helium and argon. Argon is ideal for hydrogen and oxygen detection since it has a thermal conductivity much less than the respective gases $^{45}$. Helium could be used, however due to its similar thermal 
conductivity to hydrogen, it would be unfavourable, and also yield negative peaks. Furthermore, in a photocatalysis setup, it would be useful to monitor nitrogen levels in the system. Primarily, nitrogen levels can be monitored to check for leaks in the system, but also to track whether a nitride/oxynitrides photocatalyst is degrading or not (nitrogen levels will increase in both cases).

There are two types of analysis associated with GC's; qualitative and quantitative. Data is represented as a graph of retention time (x-axis) and detector response (y-axis); the retention time is specific to each compound (qualitative), and the detector response is dependant (with a TCD) on the thermal conductivity and amount of each element (quantitative). Chromatographic data therefore yields a spectrum of 'peaks' separated only by time. The area under each peak represents the amount of substance present. By using a calibration curve (responses from a series of known concentrations/amounts), one can then determine the amount of substance present, and correctly identify the element/compound by its distinct retention time.

\subsubsection{Powder X-Ray Diffraction (PXRD)}

PXRD is used to quickly classify the atomic and molecular structure of a crystalline material, including phase, purity and composition. X-rays are fired at an (unknown) compound, causing elastic (Raleigh) scattering. Given angles of coherent and incoherent scattering are related to the lattice parameters in reciprocal space by Bragg's law ${ }^{46}$ :

$$
n \lambda=2 d \sin \theta
$$

Where $\mathrm{n}$ is an integer, $\lambda$ is the wavelength of electromagnetic radiation, $\mathrm{d}$ is the spacing between atomic planes (not atoms) and $\theta$ is the angle between atomic planes and incident x-ray angles.

By varying the angle of both the x-ray source and the detector $(2 \theta)$, it is possible to attain a relationship between intensity and scattering angle, termed a diffraction pattern. This pattern is then run through a database to quickly identify the element or compound according intensities at set scattering angles. This is an especially good technique for gaining insight into photocatalytic mechanisms, since band gaps can change with phase 
and crystallinity, and it is also very easy to see impurities, both of which can affect photocatalytic activity.

Further analysis can also yield more specific features of compounds, such as crystallite size, and the average distance between molecules. If there is a weak peak response, in conjunction with a broad width, then this is indication of poor crystallinity. However, good peak responses with broad features can often mean the particle size is very varied. The average particle or crystallite size can be calculated using Scherrer's equation ${ }^{47}$ :

Equation 19. Scherrer's equation

$$
\tau=\frac{K \lambda}{\beta \cos \theta}
$$

where $K$ is the shape factor (near 1 , but crystallite shape dependant), $\lambda$ is the $x$-ray wavelength, $\beta$ is the line broadening at half the maximum intensity, $\theta$ is the Bragg angle, and $\tau$ is the mean size of the ordered crystalline domains, which is approximately the grain size. As a side note, the Scherrer equation is only applicable to nano-sized particles, and is limited to a grain size of $0.1 \mu \mathrm{m}$. More importantly, the Scherrer formula actually calculates a lower bound to the particle size. This is because the width of the diffraction peak can be determined by many different factors, not just crystallite size, such as inhomogeneous strain and instrumental effects ${ }^{48}$. Scherrer's equation should not be used where access to more appropriate particle sizing techniques (such as SEM or TEM) are available.

\subsubsection{Scanning and Transmission Electron Microscopy}

Scanning Electron Microscopy (SEM) and Transmission Electron Microscopy (TEM) are two different techniques used for imaging the surface structure of semiconductors. SEM is often used to identify the size of particles and examine the morphology of the surface, especially with samples which are reasonably thick. The SEM produces a high energy electron beam that interacts with the surface of the sample, which in turn emits secondary electrons, backscattered and diffracted backscattered electrons, all of which are captured by detectors and are transformed into 
a high depth of field image. This gives the image a 3-dimensional appearance despite being a two dimensional image.

TEM involves the transmission of an electron beam through a thin sample $(\sim 100 \mathrm{~nm})$; the beam interacts with the sample and refracts around atoms, generating an image on a photographic film or CCD. Used mainly in imaging thin films or nanoparticles which are less than $10 \mathrm{~nm}$, it can also be used to detect nano-scale surface dopants such as metallic/crystalline elements, which often to not show on SEM image. Unlike SEM, TEM allows the examination of non-conductive sampling. If a non-conductive sample is studied using SEM, then an effect known as 'charging' occurs, often ruining the sample, and making imaging very difficult. If the CCD or photographic film is in the correct position, then it is also possible to see diffraction rings or spots from the sample, indicative of crystalline nature. If certain instrumental parameters are known, then it is possible to correlate the size of these rings with known d-spacing of the crystal structure (obtained from XRD). Then, using a diffraction pattern, it is possible to identify which crystal plane is being observed, which is especially useful when studying faceted crystals.

Scanning and Transmission Electron Microscopes often come with an additional feature: EDX (Energy Dispersive X-ray Spectroscopy, sometimes abbreviated to EDS). This analytical technique is often used for elemental analysis of samples, resulting in a spectrum of elements and their respective ratios. The electron beam from the SEM is used to stimulate ground state electrons within the sample, forcing them to be ejected. The empty ground state left behind is filled by an electron from a higher shell, and an X-ray is emitted. The emitted X-ray has energy which is equal to the difference between the two shells - which is characteristic of individual elements ${ }^{49}$. In photocatalysis studies, this can be used to identify the distribution of cocatalysts on the surface, or look at impurities.

\subsubsection{TGA-DSC-MS}

Thermo Gravimetric Analysis - Differential Scanning Calorimetry - Mass Spectroscopy (TGA-DSC-MS), is complementary set of techniques which couple thermal analysis and evolved gas analysis, essentially observing characteristic changes that a compound undergoes under varying temperature. It is particularly useful for investigating phase changes and 
condensation reactions of polymers. TGA is used to monitor mass of a solid or liquid with temperature, and with a high precision, measure mass difference due to evaporation or sublimation. This can then be plotted on a simple $\mathrm{x}-\mathrm{y}$ curve; mass loss (as a percentage) versus temperature or time. DSC gives information on thermal transitions of compounds - whether endothermic or exothermic reactions occur at a specific temperature. Again, a simple $x-y$ curve of sample heat change in comparison to a reference, versus temperature or time. MS ionises compounds from the TGA-DSC gas-solid-fragment stream and then measures their mass to charge ratio; culminating in spectra of atomic masses and their relative concentration at a given time. One can then bring the spectra together in time or temperature, and plot what masses (and thus elements or compounds) are present at that instant, and in what concentration.

\subsubsection{BET method for specific surface area measurements ${ }^{50}$}

The Brauner-Emmett-Teller (BET) theory describes the physisorption process of gas molecules on a solid surface, resulting from the relatively weak Van de Waals forces. The theory can be extended to a method for measuring the specific surface area of materials - especially relevant for photocatalysts, since a greater surface area can potentially lead to more active sites per unit area, leading to an increase in activity. Adapted from Langmuir theory, which describes the monolayer absorption of a gas on a solid, BET theory extends the concept by allowing for multilayer adsorption, and is calculated by measuring the .

Equation 20. The BET equation

$$
\frac{1}{v_{a}\left[\left(\frac{p_{0}}{p}\right)-1\right]}=\frac{(c-1)}{v_{m} c}\left(\frac{p}{p_{0}}\right)+\frac{1}{v_{m} c}
$$

Where $v_{a}$ is the volume of gas adsorbed at standard temperature and pressure (STP), $\mathrm{p}$ and $\mathrm{p}_{0}$ are the partial vapour pressure and saturation pressure of the physisorbed gas (often nitrogen, $\mathrm{N}_{2}$ ) at $\sim 195.5^{\circ} \mathrm{C}$, $\mathrm{c}$ is the BET constant, $v_{m}$ is the adsorbed gas quantity per volume to produce a monolayer.

Equation 21. The BET constant (c). 


$$
c=e^{\frac{\left(E_{1}-E_{L}\right)}{R T}}
$$

Where $E_{1}$ is the first layer's heat of adsorption, and $E_{L}$ is the corresponding heat of adsorption for $\mathbf{n}$ layers $>1$.

Equation 21 can be plotted simply as a ' $y=m x+c$ ' graph, the result termed a 'BET plot'. The ordinate term is $\frac{1}{\left.v_{a}\left[\frac{p_{0}}{p}\right)-1\right]}$, whilst $\left(\frac{p}{p_{0}}\right)$ can be plotted on the abscissa. Linearity is found at approximately $0.01 \leq\left(\frac{p}{p_{0}}\right) \leq$ 0.3 and thus extrapolating from this point using the gradient $\mathrm{A}$ to $\mathrm{y}$ intercept $I$ gives $v_{m}$ and $c$ according to the equations:

Equation 22.

$$
v_{m}=\frac{1}{A+I}
$$

Equation 23.

$$
\mathrm{c}=1+\frac{\mathrm{A}}{\mathrm{I}}
$$

The BET specific surface area (SSA) is then given by:

Equation 24. BET SSA equation

$$
S_{B E T}=a\left(\frac{v_{m} N_{A} S}{V}\right)
$$

Where $a$ is the mass of the powdered adsorbant, $\mathrm{N}_{\mathrm{A}}$ is Avogadro's number, $\mathrm{s}$ is the adsorption cross section of the powder, and $\mathrm{V}$ is the molar volume of gas.

From a practical point of view it is in fact much simpler to attain a value for specific surface area, since software accompanies the surface area analyser which can calculate all of the above automatically. All that is required is a precise measurement of the mass of the sample, and to correctly pick the point of linearity. Linearity (a line of best fit) can be improved by a constant trial and error process which minimises the correlation coefficient ( $\mathrm{R}$-factor), so that the value is as close to $\mathbf{1}$ as possible (acceptable values are normally $\geq 0.999$ ). 


\subsubsection{Zeta Potential (ZP) using Electrophoretic Light Scattering $(\text { ELS })^{51}$}

In a colloid, microscopic particles are suspended in a solvent by nature of their ZP; the potential difference between the solvent and a fixed layer of solvent attached to the dispersed particle. The electrokinetic properties are determined by the electric charge distribution at the 'double layer' surrounding the particle. When immersed in an electrolyte such as water, an ionic particle becomes surrounded with counter ions, with a charge opposite to that at the surface. The first layer is formed from chemical interactions, whilst the second layer that comprises the double layer is attracted by nature of the Coulomb force, and thus electrically screens the first layer. The second layer (slipping plane) is only lightly bound to the particle and is made of free ions, and thus its depth can be influenced by the amount of positive or negative charge within the solution. The potential at the slipping plane is the zeta potential, as measured from the surface of the particle.

In short, zeta potential determines the stability of the colloid, as highly charged particles repel each other, and prevent flocculation. As a rule of thumb, a colloid with a $\mathrm{ZP}$ of $0 \mathrm{mV}$ (Isoelectric Point, IEP) will undergo rapid coagulation or flocculation, $\sim 40 \mathrm{mV}$ will demonstrate good stability, and $\geq 60 \mathrm{mV}$ exhibits excellent stability.

A zeta potential measurement not only provides information of the stability of a colloid, but upon further analysis, can give relative information about the surface charge - whether positive or negative, and relative magnitude. Its application to photocatalysis stems really from the latter point; the information gained regarding surface charge can help to explain phenomena experienced in synthesis methods, and more importantly, differences in activity due to surface species such as $\mathrm{H}^{+}$and $\mathrm{OH}^{-}$.

One effective way of measuring zeta potential is to use microelectrophoresis (rate of particle movement under an electric field) in conjunction with electrophoretic light scattering. The particle-electrolyte colloid is placed in a specialised cuvette-type cell, an oscillating electric field is then applied, which causes the particles to move with a velocity proportional to their zeta potential. In order to measure this velocity, a laser beam is passed through the cell, and the Doppler shift frequency observed is proportional to the dispersed particle's mobility according to: 
Equation 25.

$$
v_{D}=\mu \frac{n E}{\lambda_{0}} \sin \theta
$$

Where $v_{d}$ is the Doppler shift frequency, $n$ is the refractive index of the medium, $\lambda_{0}$ is the wavelength of incident light in vacuum, and $\theta$ is the scattering angle.

The electrophoretic mobility $\mu$ is defined as:

Equation 26.

$$
\mu=\frac{v}{E}
$$

Where $v$ is the velocity of the particles, and $\mathrm{E}$ is the electric field applied.

Electrophoretic mobility is directly proportional to zeta potential:

Equation 27:

$$
\mu=\frac{\varepsilon_{0} \varepsilon_{r} \zeta}{\eta}
$$

Where $\varepsilon_{0}$ is the permittivity of free space, $\varepsilon_{\mathrm{r}}$ is the permittivity of the suspension medium, and $\eta$ is the viscosity of the suspension medium.

Therefore by measuring velocity, under a known electric field, and having known constants (often pre-programmed into the apparatus), one can attain the zeta potential. 


\subsubsection{Attenuated Total Reflectance - Fourier Transform InfraRed (ATR-FTIR) spectroscopy ${ }^{52}$}

'ATR-FTIR' is a fast infra-red spectroscopic technique for probing the local surface structure of a material, with minimal preparation. From an analysis point of view, infrared spectroscopy enables the detection and fingerprinting of covalent bonds. This is useful for both characterisation and analysis of impurities/contaminants on the surface of semiconductor photocatalysts. Since most photocatalysts are crystals with simple molecular structures, they possess few IR-active bonds. However, organic contaminants or impurities are intrinsically more molecularly complex, and thus will easily be detectable using IR spectroscopy.

In IR spectroscopy, a sample is illuminated with a monochromatic light source. Absorption will occur if the frequency of light corresponds to the vibrational frequency of the bond; this is then apparent the in the spectrum of transmittance, and can be correlated to how much energy has been absorbed. FTIR is more counter-intuitive, yet has a much faster sampling time and higher signal to noise ratio ${ }^{53}$. In FTIR, a broadband/polychromatic light source (all frequencies to be measured) is used to illuminate the source. However, the beam first passes through a Michelson interferometer, a beam splitting device which causes a deliberate path length shift (termed 'retardation'), subsequently illuminating the detector after hitting the sample with a mixed interference beam of all wavelengths. Beam retardation is caused by a moving mirror within the interferometer, which has real units of length. Therefore the detector produces an interferogram; a fixed set of retardation values and a corresponding set of intensities. A Fast Fourier Transform (FFT) is automatically applied by the instrument, converting time/space to frequency. The resultant FTIR spectrum is a set of inverse lengths $\left(\mathrm{cm}^{-1}\right.$ is often used) versus intensity. Bonds can thus be assigned to intensities at certain energies (wavenumbers).

ATR-FTIR is similar in respect to standard FTIR methods, however it has considerable advantages in terms of preparation method, and is now gaining a considerable hold on the academic market. A solid or liquid can simply be placed on the ATR crystal with a small amount of pressure to form an intimate contact, and then analysed. Compared to traditional FTIR methods, which rely on transmission and therefore a diluted sample/thin film, ATR-FTIR relies on Total Internal Reflectance (TIR) and thus does not have this issue. The sample is also then reusable, and uncontaminated. 
The beam of infrared light is incident on the ATR crystal; passing through and reflecting off the sample (see Figure 7). As a result of TIR, an evanescent wave is set up, propagating through the crystal and reflecting of both crystal and sample consecutively ${ }^{54}$. As long as the ATR crystal has a higher refractive index than the sample, the technique is viable. The penetration depth into the sample is approximately $0.5-2 \mu \mathrm{m}$.

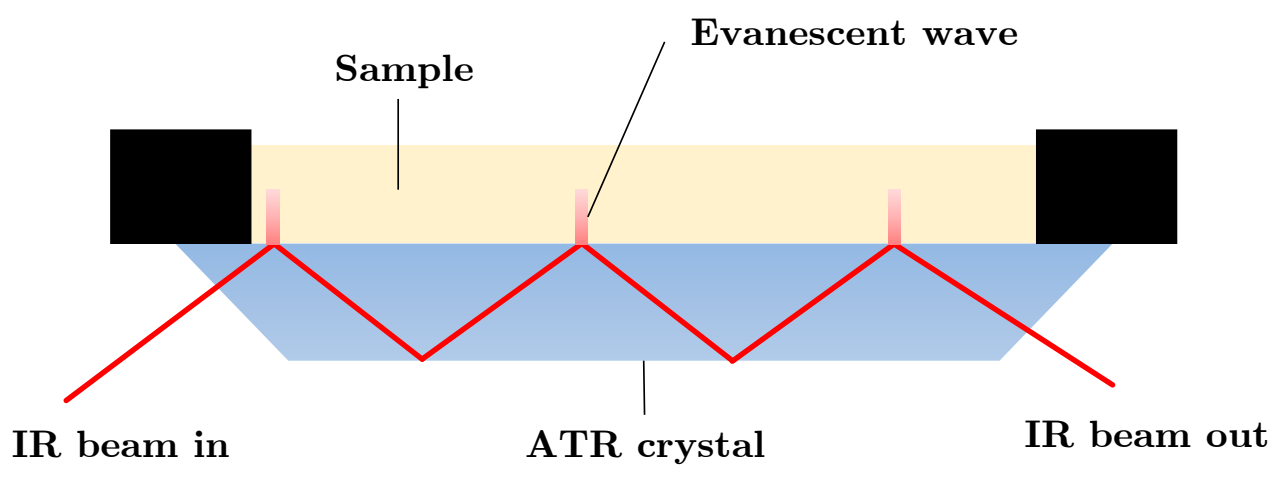

Figure 7. Schematic demonstration of a working ATR crystal in an ATRFTIR spectrometer ${ }^{52}$.

\subsubsection{Raman Spectroscopy}

Complementary to FTIR spectroscopy in the sense that it can provide a compounds fingerprint, Raman spectroscopy is less widely used method of detecting vibrational, rotational and other low frequency modes in a compound $^{55}$. In FTIR spectroscopy, a polychromatic light source is used, and detection of activated IR bonds depends on the loss of a certain frequency due to absorption. In Raman spectroscopy, a monochromatic source illuminates the sample, which is in turn scattered by the sample; it is not an absorption technique. An incident photon polarises the electron cloud and creates an inherently unstable excited virtual state, which is then stabilised by reemitting another photon of similar, but not identical energy. Figure 8 illustrates this process. A degree of electron cloud deformation (polarizability) caused by the photon with regards to the vibrational coordinate is necessary for a molecule to undergo a Raman effect. The magnitude of polarizability is proportional to the Raman scattering 
intensity. The dependence on the polarizability is thus the difference between Raman and IR spectroscopy, as IR spectroscopy only probes molecules whereby photons interact with the molecular dipole moment. This enables Raman spectroscopy to analyse vibrational/rotational transitions which are effectively not allowed as determined by the rule of mutual exclusion in centrosymmetric molecules ${ }^{56}$.

Elastic/Raleigh scattering occurs when the photon excites a ground state vibrational mode to a virtual state and subsequently returns to the same energy ground state. Common Raleigh scattering is around $10^{6}-10^{8}$ more prevalent than Raman scattering, which is a very weak process, and thus needs a monochromatic source.

Raman scattering can be divided into two different types of scattering; 'Stokes' and 'Anti Stokes'. 'Stokes' scattering arises when a photon excites a ground state vibrational mode $\left(\mathrm{n}_{0}\right)$ to an initial virtual state $\left(\mathrm{v}_{1}\right)$, and is then reemitted with a lower energy, the difference in photon energy is thus the difference between the vibrational energy states $\left(n_{1}-n_{0}\right)$. Some molecules however may be in an excited state (from thermal energy), thus 'Anti Stokes' scattering takes place when the incident photon excites a higher energy vibrational state $\left(n_{1}\right)$ to a higher virtual state $\left(v_{2}\right)$, which then returns to the ground state $\left(\mathrm{n}_{0}\right)$. The energy of the virtual states are determined by the power of the incident photon.

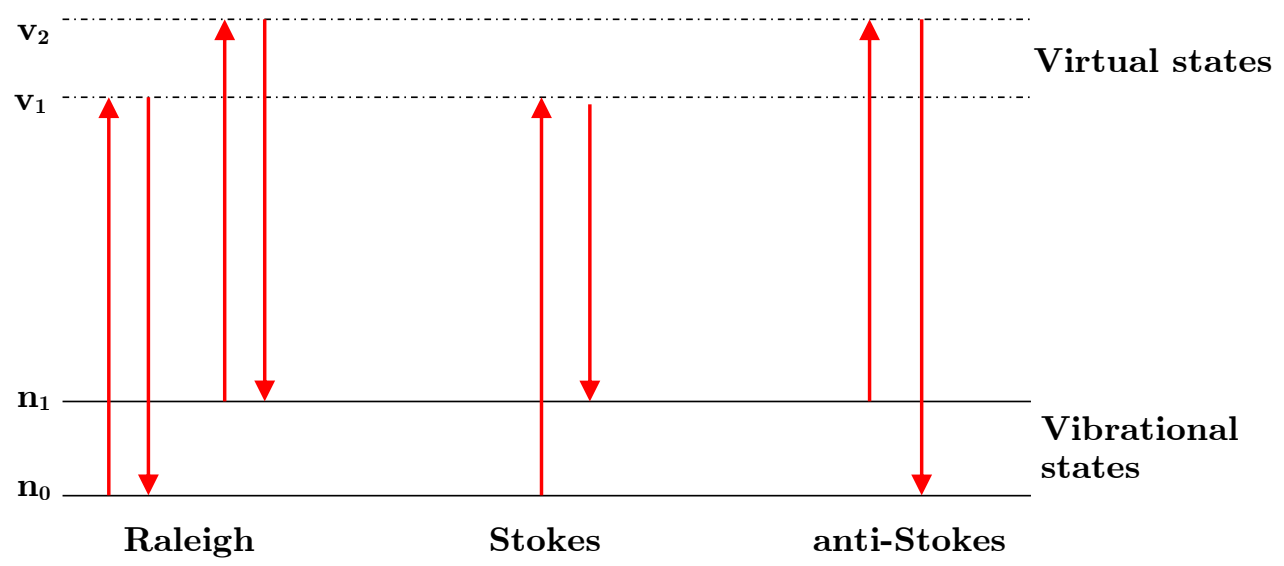

Figure 8. Raleigh and Stokes/Anti Stokes scattering processes in diagram form. Lowest energy vibrational state is $\mathbf{n}_{0}$, and highest virtual states is $\mathbf{v}_{2}$. 
Arrows represent direction of photon excitation (upwards) and relaxation (downwards).

Practically, Raman spectroscopy is relatively quick, there is little sample preparation (powdered samples are simply put in the way of a laser beam), and is non selective. The monochromatic laser light is Raman scattered by the sample, collected by a lens and focused onto a detector. Photons which are Raleigh scattered (i.e. no energy change) are rejected by use of a notch or edge filter. The software within the instrument then compares the initial laser wavelength $\left(\lambda_{0}\right)$ with the Raman scattered wavelength $(\lambda)$ and calculates the Raman shift $\left(\lambda_{0}-\lambda\right)$. The generated plot is thus a function of Raman shift $\left(\mathrm{cm}^{-1}\right)$ versus intensity, and shows what vibrational/rotational bonds are present in the molecule/sample.

In terms of its applicability to analysing semiconductor photocatalysts, Raman spectroscopy can be used in two different ways. The first is simply to detect possible contaminants on the surface of a semiconductor photocatalyst, and correlate with photocatalytic activity. Often, contaminants act as either recombination traps or electron/hole scavengers, and having a detrimental effect on photocatalytic activity by preventing the reduction or oxidation of water. Secondly, since Raman spectroscopy probes local molecular structure through the Raman shift, a change in Raman shift at a particular wavelength would infer that the bond is either moving up or down in terms of energy. It could therefore be interpreted that an increase or decrease in bond energy results in a distortion in local structure (i.e. change in electron orbital overlap), which can result in either a decrease or increase in photocatalytic activity, as demonstrated with $\mathrm{BiVO}_{4}$ by $\mathrm{Yu}$ et $\mathrm{al}^{31}$.

\subsubsection{X-ray Photoelectron Spectroscopy (XPS)}

XPS enables the characterisation of the electron energy distribution within a compound by irradiation with an x-ray source ${ }^{57}$. Primarily a qualitative surface sensitive elemental analysis technique, XPS is capable of measuring atomic composition (\%), chemical and electronic state, and bonding configurations of elements in a sample, all whilst being completely nondestructive. Depth profiling deeper into the bulk is possible using ion etching, but native use is limited up to approximately $10 \mathrm{~nm}$ into the bulk from the surface ${ }^{58}$. Electrons in shells up to but not including the valence 
band can be excited by x-rays, whilst valence electrons are excited by UV light, and measured using a similar technique, Ultraviolet Photoelectron Spectroscopy (UPS). Both techniques must be under Ultra High Vacuum (UHV) in order for the electrons to reach the detector. Most elements can be quantitatively and qualitatively measured, with the exception of hydrogen and helium - the diameter of the orbitals are too small therefore the catch probability is close to zero ${ }^{59}$.

When a sample is irradiated with a high energy x-ray source (ca. $1.4 \mathrm{keV}$ for a common aluminium K-alpha source), core level electrons are excited to the vacuum level and are then detected by an electron energy analyser. The binding energy $\left(E_{b}\right)$ of the core level is then calculated using the following formula:

Equation 28. Conservation of energy

$$
E_{b}=E_{\gamma}-\left(E_{K E}+\phi\right)
$$

Where $E_{\gamma}$ is the energy of the incident $\mathrm{x}-\mathrm{ray}, \mathrm{E}_{\mathrm{KE}}$ is the measured energy of the electron measured by the instrument, and $\phi$ is the work function of the instrument.

As incident electrons hit the detector, both electron intensity and binding energy are recorded as a peak-like spectrum, often with a sizeable background. Since the core level binding energies in an atom are unique to each element, it is initially possible to identify the element and electron orbital. Further peak analysis can then separate each individual contribution to that element, for example, a carbon 1s peak might have convolutions of 2 or 3 different bonds in different binding energies; C-C, C$\mathrm{O}$ or $\mathrm{O}-\mathrm{C}=\mathrm{O}^{57}$.

XPS has considerable applicability in the characterisation of photocatalysts, evidenced by its importance in recent high profile articles ${ }^{60,61,62}$. Since numerous UV-active photocatalysts are cheap, robust, and relatively efficient (discussed in section 2.3), many research groups have actively tried to extend their absorption into the visible region so that more light can be absorbed and increase the potential STH\%. One of the ways is to use dopants - ions which alter the band structure and effectively insert a subband gap state to enable more light absorption. Monitoring the atomic percentage of these dopants after preparation is useful in order to ascertain the optimum dopant percent - this can effectively be done with XPS. In 
addition, using XPS depth profiling combined with traditional electron orbital labelling, it is possible to differentiate between surface species and bulk-bound ions through location mapping and oxidation state identification ${ }^{63}$.

\subsubsection{Elemental Analysis (EA)}

EA, or in the most common and applicable form, CHNX analysis, is the quantitative and qualitative determination of the elemental composition and weight of a compound by combustion. Often used to determine carbon, hydrogen, and nitrogen, in organic molecules, other compounds can also be detected using additional gas separation methods. Put simply, a compound of unknown composition is combusted in an oxygen rich atmosphere, the constituents separated by gas chromatography, and in modern apparatus, analysed using a high temperature $\mathbf{T C D}^{64,65}$.

A small amount (ca. 10mg) of sample is weighed in a small aluminium capsule. The $\mathrm{Al}$ capsule is then moved to a high temperature furnace (in some cases, over $1800{ }^{\circ} \mathrm{C}$ ) and combusted under a pure static oxygen atmosphere. An extra purge of oxygen is then added at the end to facilitate complete combustion. The produced gases are then passed through reagents that produce more stable gases such as $\mathrm{CO}_{2}, \mathrm{H}_{2} \mathrm{O}$ and $\mathrm{N}_{2}$. This step also removes phosphorus, sulphur and halogen based compounds. In order to 'scrub' oxygen and oxides, the gases then pass over a copper section, which reduces oxides of nitrogen to elemental nitrogen. The gas is then homogenized at a constant temperature and pressure when passed through a mixing volume chamber.

The gas is then passed through a series of TCDs, with $\mathrm{H}_{2} \mathrm{O}$ traps in between. The difference between the two signals as a loss of water is thus proportional to the hydrogen concentration in the sample being analysed. Another set of TCDs with a $\mathrm{CO}_{2}$ trap in between calculate the carbon concentration, and finally nitrogen is measured using a helium reference over a TCD. Other compounds, such as sulphur and oxygen can be analysed using specialised reaction tubes.

The atomic weight of $\mathrm{C}, \mathrm{H}$ or $\mathrm{N}$ is useful for analysis of semiconductor elements containing the three elements. In particular, the composition of graphitic carbon nitride (one of the only organic semiconductor photocatalysts) can be analysed, and the carbon to nitrogen ratio calculated. 


\subsection{Overview of current photocatalysts ${ }^{66}$}

This subsection draws on relevant publications in the field, ranging from well documented literature, to newer, novel findings and alternative approaches to achieving photocatalytic water splitting with high efficiencies. By studying reviews both old and new, inspiration can be drawn for future novel photocatalysts, improving efficiencies and creating new production methods. It is noted that the review will not focus on dyesensitization, as this is out of the scope of the project due to long term feasibility issues associated with the stability of most well-known light absorbing dyes ${ }^{67}$. Furthermore, PEC systems, or coupled systems (photocatalyst + solar cell) will not be considered due to their complexity, and less feasible nature for this project which is to screen efficient materials ${ }^{68}$.

\subsubsection{UV-active semiconductors}

In 1972, Honda and Fujishima showed that $\mathrm{TiO}_{2}$ could be used to split water when irradiated with UV-light. They used a PEC cell, with Pt as the counter electrode, and an external bias. Hydrogen was evolved on the Pt electrode, and oxygen on the $\mathrm{TiO}_{2}$ electrode ${ }^{6}$. After this experiment, extensive research began on water splitting, in an effort to generate a more efficient photocatalyst which could readily produce clean, renewable fuel. $\mathrm{TiO}_{2}$, the quintessential photocatalyst, has been continually investigated through various methods, including doping with different elements, altering particle size and morphology, and using different cocatalysts. In the mid 80's, many groups began changing experimental conditions and adding in various cocatalysts to improve performance. Yamaguti and Sato found that by loading $\mathrm{TiO}_{2}$ with $\mathrm{Rh}$ and using $\mathrm{NaOH}$, an enormous increase in gas evolution rates could be achieved; with a QY of $29 \%$ at $365 \mathrm{~nm} .{ }^{69}$. Selli et al. reported enhanced performance when a $\mathrm{pH}$ difference was employed between a $\mathrm{TiO}_{2}$ electrode and $\mathrm{Pt}$ counter electrode ${ }^{70}$. At a similar time, Gratzel's group reported a series of experiments which focused on a $\mathrm{TiO}_{2}$ colloid loaded with $\mathrm{Pt}$ and $\mathrm{RuO}_{2}$ as a reduction and oxidation catalyst respectively. They found this further increased the quantum yield, and also increased the hydrogen evolution rate $^{71}$. Hydrogen was found to be produced at $2.8 \mathrm{~cm}^{3} \mathrm{~h}^{-1}$, much higher compared to $1 \mathrm{~cm}^{3} \mathrm{~h}^{-1}$ without $\mathrm{RuO}_{2}{ }^{72}$. Oxygen gas was not detected at the beginning of the experiment because it 
was assumed to strongly adhere to the catalyst in the initial period. Instead of using anatase $\mathrm{TiO}_{2}, \mathrm{Fu}$ et al studied bicrystalline titania $\left(\mathrm{TiO}_{2}(\mathrm{~B})\right)^{73}$. They synthesised and investigated a heteropoly-blue sensitizer and $\mathrm{Pt}$ loaded $\mathrm{TiO}_{2}(\mathrm{~B})$ nanoribbon for water decomposition for hydrogen evolution (quantum yield $(\mathrm{QY})=\mathbf{8 . 1 1 \%}$ ). Their result showed that a mixture of $\mathrm{TiO}_{2}(\mathrm{~B})$ and anatase gave the maximum quantum yield, suggesting that interfacial charge separation improved the efficiency. In another report by this group, the solvent effect of water splitting was investigated ${ }^{74}$. The hydrogen production rate was found to be increased in the presence of monochloroacetic acid and dichloroacetic acid with Pt/P25 as the photocatalyst. $\mathrm{TiO}_{2}$ remains limited by its large band gap which prohibits light absorption in the visible range. Despite efforts to alter its characteristics, it still yields low efficiencies as dopants only offer a limited number of donor/acceptor states in comparison to the large number of states available at Ti3d and $\mathrm{O} 2 \mathrm{p}$ orbitals ${ }^{60}$. Scientists have also experimented with other UV materials which share similarities with $\mathrm{TiO}_{2}$ and explored their characteristics. Metal oxides which respond to UV light commonly have a full valence band which is mainly constructed of oxygen $2 p$ electron orbitals, and a conduction band with empty metal d orbitals for transition metals and $s$ or $p$ orbitals for main group metals.

Domen's group paid particular attention to $\mathrm{SrTiO}_{3}$ - a perovskite structure material, part of the 'titanates'75,76,77,78,79. $\mathrm{SrTiO}_{3}$ has a band gap of $3.2 \mathrm{eV}$, so responds to $\mathrm{UV}$ light $(\lambda<387 \mathrm{~nm})$. They examined the use of $\mathrm{NiO}$ as a cocatalyst, and found that both oxygen and hydrogen can be evolved in a stoichiometric ratio - pure water splitting. The $\mathrm{NiO}$ cocatalyst was prepared by a $\mathrm{H}_{2} / \mathrm{O}_{2}$ redox reaction on $\mathrm{NiO}$ to form a $\mathrm{Ni} / \mathrm{NiO}_{\mathrm{x}}$ double layer. $\mathrm{Kim}$ et al. studied another important titanate, $\mathrm{La}_{2} \mathrm{Ti}_{2} \mathrm{O}_{7}$. Lanthanum titanate is a layered structure consisting of four $\mathrm{TiO}_{6}$ unit slabs separated by $\mathrm{La}^{3+}$ ionic layers ${ }^{80}$. When $\mathrm{La}_{2} \mathrm{Ti}_{2} \mathrm{O}_{7}$ splits water, $\mathrm{H}_{2}$ and $\mathrm{O}_{2}$ can be produced in high amounts (QY up to $12 \%$ ) in the presence of a $\mathrm{NiO}$ cocatalyst. It can be even further enhanced by adding $\mathrm{BaO}$ as a dopant, and increasing $\mathrm{pH}$ with $\mathrm{NaOH}$; the QY increases to $50 \%$.

Rutile zirconia $\left(\mathrm{ZrO}_{2}\right)$ has a very large band gap (5.0-5.7V), but can split water without a cocatalyst under UV illumination ${ }^{81}$. Sayama and Arakawa reported the use of alkali carbonates in water; $\mathrm{NaHCO}_{3}$ gave the highest efficiency in water splitting ${ }^{82}$. Interestingly, here, the addition of a cocatalyst actually decreased the ability of $\mathrm{ZrO}_{2}$ to split water. This suggests that there is a large energetic barrier between the cocatalyst and semiconductor as a result of the large bandgap and position of the $\mathrm{CB}$ and 
VB. Jiang et al turned their attention to $\mathrm{ZrW}_{2} \mathrm{O}_{8}$ in order to overcome the problem of $\mathrm{ZrO}_{2}$ having large band gap ${ }^{83} \cdot \mathrm{ZrW}_{2} \mathrm{O}_{8}$ was estimated to have a band gap of about $4.0 \mathrm{eV}$. It showed ability to decompose water to yield hydrogen or oxygen in the presence of an appropriate electron or hole scavenger under a $300 \mathrm{~W} \mathrm{Hg}$-Xe lamp. The production rate was not high, e.g. $23.4 \mu \mathrm{mol} \mathrm{h}^{-1}$ for $\mathrm{H}_{2}$ and $9.8 \mu \mathrm{mol} \mathrm{h} \mathrm{h}^{-1}$ for $\mathrm{O}_{2}$.

Tantalates and niobates have shown some of the highest efficiencies recorded for photocatalysis. A large number of reports focus on combining an alkaline earth metal with a tantalate: ( $\mathrm{M}$ represents different alkaline metals; $\mathrm{Na}, \mathrm{K}, \mathrm{Rb}$, etc) $\mathrm{MTaO}_{3}{ }^{84,85,86}, \mathrm{MTa}_{2} \mathrm{O}^{87}, \mathrm{M}_{2} \mathrm{Ta}_{2} \mathrm{O}_{7}^{88}$, and $\mathrm{M}_{2} \mathrm{Nb}_{2} \mathrm{O}_{7}{ }^{89}$. These materials are capable of producing both $\mathrm{H}_{2}$ and $\mathrm{O}_{2}$, but only when illuminated with high energy UV light. The perovskite structure, as seen in $\mathrm{SrTiO}_{3}$, is also present within both tantalates and niobates; the $\mathrm{ABO}_{3}$ structure allows a wide range of options to be explored, as many different metals can fit into the $\mathrm{A}$ and/or $\mathrm{B}$ sites. It was reported that sodium tantalate $\left(\mathrm{NaTaO}_{3}\right)$ exhibits a particularly high quantum yield $(20 \%, \lambda=$ 270nm) for both hydrogen and oxygen production, when used in conjunction with a $\mathrm{NiO}$ cocatalyst ${ }^{87}$. In further work, it was found that doping the $\mathrm{NaTaO}_{3} / \mathrm{NiO}$ compound with lanthanum (2 mol\%) resulted in the quantum yield increasing to $56 \%$, the current record for UV driven water splitting. It is believed that the addition of La not only reduces the particle size (increasing the overall specific surface area), but also helps to separate reduction and oxidation sites.

$\mathrm{M}_{4} \mathrm{Nb}_{6} \mathrm{O}_{17}$, layered niobate structures, were reported to be useful for overall water splitting ${ }^{84}$. It has been suggested that these structures enable the production of both $\mathrm{H}_{2}$ and $\mathrm{O}_{2}$ in two different layers. This would be an advantageous technique for splitting water - as separate evolution sites are known to prevent recombination, but whole separate layers would inhibit recombination even further. Although $\mathrm{M}_{4} \mathrm{Nb}_{6} \mathrm{O}_{17}$ materials are able to split water without any cocatalyst at relatively low efficiencies, it was reported that hole scavengers can increase $\mathbf{H}_{2}$ production dramatically ${ }^{90}$.

Studies have also been carried out regarding the comparison of lattice parameters regarding niobate and tantalate perovskites ${ }^{91}$. The authors claim that phonon modes in semiconductors play a vital part in regulating the migration of charge carriers, and can be the deciding factor concerning performance. A relationship between Raman spectra results and the catalytic performance shows that a red shift in phonon frequency leads to a decrease in activity, essentially overriding influences of particle size and 
band gap width. Perovskites have the ability to accept many different metal dopants, and efforts are being made to enhance not just UV-active materials, but their visible light driven counterparts as well. 
2.3.2 Semiconductors activated by visible light.

Metal oxides in the main group of the periodic table have also been subject to extensive research for the purpose of water splitting. Elements in groups 13 and 14; In, Sn, Sb, Ga and Ge have all been reported to be active for water splitting when combined with suitable cocatalysts. Sato et al reported $\mathrm{MIn}_{2} \mathrm{O}_{4}$ (in this case $\mathrm{M}$ represents $\mathrm{Ca}, \mathrm{Sr}$ and $\mathrm{Ba}$ ) had distorted $\mathrm{InO}_{6}$ octahedra in the lattice, showing $\mathrm{H}_{2}$ and $\mathrm{O}_{2}$ production from pure water when a cocatalyst $\left(\mathrm{RuO}_{2}\right)$ was added to the materials, through an impregnation method ${ }^{92,93}$. $\mathrm{CaIn}_{2} \mathrm{O}_{4}$ exhibited the highest activity under UV light. Tang et al examined different $\mathrm{MSnO}_{3}(\mathrm{M}=\mathrm{Ca}, \mathrm{Sr}$ and $\mathrm{Ba})$ colloids, and obtained both hydrogen and oxygen in the presence of the $\mathrm{RuO}_{2}$ cocatalyst ${ }^{94} . \mathrm{SrSnO}_{3}$ has the highest photocatalytic activity among common tin oxides, due to the suitable band position and rapid charge transfer, mainly due to the distorted connection of $\mathrm{SnO}_{6}$ in $\mathrm{SrSnO}_{3}$, and also due to the strong electron-lattice interaction. The study of the $\mathrm{SrSnO}_{3}$ morphology found that preparation method can lead to very different morphologies, which in turn alter performance. $\mathrm{SrSnO}_{3}$ nanorods prepared by a hydrothermal method led to a $\mathbf{1 0}$ fold increase in photocatalytic activity, in comparison to standard spherical particles (high temperature solid state reaction) which have similar surface area and optical density ${ }^{95}$. Sato et al. have also found the ability of $\mathrm{M}_{2} \mathrm{Sb}_{2} \mathrm{O}_{7}$ structures $(\mathrm{M}=\mathrm{Ca}, \mathrm{Sr})$, which also contain octahedral $\left(\mathrm{SbO}_{6}\right)$ connections, to split water into hydrogen and oxygen in near-stoichiometric ratio under UV radiation and with the addition of $\mathrm{RuO}_{2}{ }^{96}$. Gallium and germanium oxides have also been explored; water splitting is possible with $\mathrm{Ga}_{2} \mathrm{O}_{3}$ and $\mathrm{Zn}_{2} \mathrm{GeO}_{4}$. Interestingly, reports show that doping $\mathrm{Ga}_{2} \mathrm{O}_{3}$ with $\mathrm{Zn}$ can result in increases of up to 8 times in photocatalytic performance ${ }^{97,98,99}$.

Metal sulphides and nitrides also have the ability to dissociate water. The valence bands are often sulphur $3 p$, or nitrogen $2 p$, which results in a contraction of band gap as S3p and N2p orbitals often lie at more negative potentials than that of O2p. ZnS has been one of the most extensively tested materials from the non-oxide group, even though its $3.6 \mathrm{eV}$ band gap prohibits visible light absorption. Yanagida et al. reported hydrogen evolution using a relatively weak $125 \mathrm{~W} \mathrm{Hg}$ lamp, and used the relatively uncommon hole scavenger, tetrahydrofuran ${ }^{100}$. Another report changed a great deal of variables, and methodically examined the photocatalytic activity of zinc sulphide; altering the hole scavenger, $\mathrm{pH}$ and temperature. A record $\mathbf{9 0 \%}$ quantum yield was attained for hydrogen production, at 313 $\mathrm{nm}$ with a platinum cocatalyst, immersed in a solution of $\mathrm{Na}_{2} \mathrm{~S}, \mathrm{H}_{3} \mathrm{PO}_{3}$ and 
$\mathrm{NaOH}^{101}$. Fujishima's group evolved hydrogen from the semiconductors $\mathrm{CuInS}_{2}$ and $\mathrm{CuIn}_{5} \mathrm{~S}_{8}$. They were suspended in water with a sulphite hole scavenger under UV illumination ${ }^{102}$. However sulphides often undergo selfdegradation via photocorrosion (typically photooxidation of sulphur ions), therefore it is vital that a sulphite/sulphate scavenger is used to replenish the degraded semiconductor.

A string of reports have been published on UV responsive nitride photocatalysts, investigating the feasibility and exploring the band structure ${ }^{103,104,105,106,107}$. The DOS reports suggest valence bands are made up of nitrogen $2 p$ electron orbitals. This has the effect of narrowing the band gap. $\mathrm{Ge}_{3} \mathrm{~N}_{4}$ was the first non-oxide, UV-active material for overall water splitting. Its VB is structured by N2p orbitals, and its CB is a hybrid of Ge4s4p. The reported quantum yield is $9 \%$ at $300 \mathrm{~nm}$ when used in conjunction with a $\mathrm{RuO}_{2}$ cocatalyst. Gallium Nitride (GaN) is already a widely used material in blue LED's, violet laser diodes, and high speed field effect transistors. It has a band gap of $3.4 \mathrm{eV}$, which enables it to only effectively use radiation with $\lambda<365 \mathrm{~nm}$. Water splitting is possible using GaN (with a Rh - Cr cocatalyst complex), however, when combined with $\mathrm{ZnO}$ in a solid solution - another UV-active compound, the joint GaN:ZnO solid solution (achieved due to same wurtzite structure and similar lattice parameters) responds to visible light. Computational studies present strong evidence that this is due to p-d repulsion in electron orbitals ${ }^{108}$, which can be found in O2p - Zn3d and it is hypothesised that this same phenomenon appears in N2p - Zn3d orbitals; essentially moving the valence band upwards to higher potentials - narrowing the band gap to around 2.4 - 2.8 eV. Even though nitrides and sulphides somehow suffer from photocorrosion, research is still active, since a lot can still be learnt through experimentation - evident for example from $\mathrm{GaN}: \mathrm{ZnO}$ research. 
2.3.2 Semiconductors activated by visible light

To reiterate, an efficient photocatalytic system for water splitting is the target in the field, as visible light offers enough energy to meet industrial efficiencies. It is widely accepted a single photocatalyst for both $\mathrm{H}_{2}$ and $\mathrm{O}_{2}$ production from water is preferable due to its simplicity, however since that goal is considered by some to be extremely difficult to attain, significant developments have been made in designing photocatalysts that are active for half reactions - either hydrogen or oxygen. These photocatalysts, if active enough, can be used in Z-Schemes for overall water splitting, or to produce hydrogen by utilising chemical feedstocks and biomass in half reactions ${ }^{109,110,111}$. Oxygen produced from photocatalytic half reactions can also be used as a pure $\mathrm{O}_{2}$ source for fuel cells. However, in comparison to UV active photocatalysts, the number of pure phase undoped visible light active photocatalysts are few in number, with even less showing reasonable activity for half reactions and good stability.

Usually, stable semiconductor photocatalyst metal oxides are composed of O2p orbitals and transition metal $\mathrm{d}^{0}$ or $\mathrm{d}^{10}$ shells, with approximate band gaps around $3 \mathrm{eV}$ due to the valence band position. Despite having the necessary redox potentials to prevent photocorrosion, UV-active photocatalysts are unable to provide the necessary efficiency required for industrial application. Therefore, one of the first methods researchers used to reduce the band gap of UV-active materials was to dope anions (e.g. N, $\mathrm{C}$ and $\mathrm{P}$ ) or cations (e.g. $\mathrm{Cr}, \mathrm{Ni}, \mathrm{Ta}, \mathrm{Sb}, \mathrm{Fe}$ ) to provide new valence and conduction band positions (Figure 9).

Photocatalysts such as $\mathrm{TiO}_{2}$ have been extensively doped since 1982, Borgarello et al pioneered a pentavalent chromium dopant which enabled the production of both hydrogen and oxygen under visible light $(400 \leq \lambda \leq$ $500 \mathrm{~nm})^{112}$. Afterwards, various groups doped $\mathrm{TiO}_{2}$ with metals such as $\mathrm{V}$, $\mathrm{Mn}, \mathrm{Mo}, \mathrm{Fe}, \mathrm{Ni}, \mathrm{Sn}$, etc, however, results show that quantum yields for any discrete visible light wavelength range did not exceed $1 \%^{113,114,115,116,117}$. In some cases, metal ion species act as both light harvesting centres (band gap change), and also as electron/hole traps, preventing recombination. This is especially evident when $\mathrm{Ag}^{+}$and $\mathrm{Pt}^{4+}$ were used and activity increased considerably. ${ }^{118,119}$ On the contrary, certain metal ions such as $\mathrm{Co}$ and $\mathrm{Al}$ have a detrimental effect on photocatalytic activity, presumably acting as recombination centres and preventing charge transfer to the surface ${ }^{117}$. The prototypical non-metal doping of titania was demonstrated by Asahi, who successfully increased the absorption spectrum to over $500 \mathrm{~nm}$ by doping 
with nitrogen ${ }^{60}$. Unfortunately, water splitting is not possible with $\mathrm{N}-\mathrm{TiO}_{2}$, as the kinetic requirements for oxidation are not satisfied by the potential of the N2p dopants. Organic degradation is however possible, and many reports have given evidence to the mechanisms behind the enhancement ${ }^{42,61}$. Various other dopants such as sulphur, carbon, boron, and fluorine among others are able to be successfully incorporated into the crystal structure of $\mathrm{TiO}_{2}{ }^{120,121,122}$, however all displaying quantum efficiencies of less than $1 \%$ at visible light wavelengths.

\section{Conduction band}
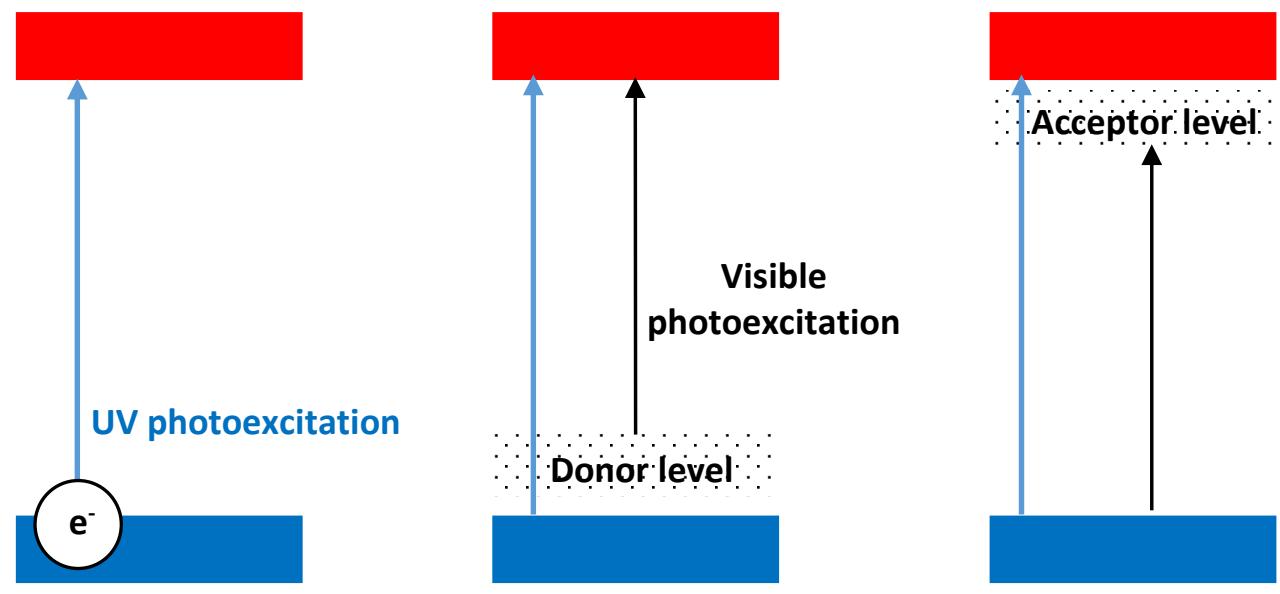

\section{Valence band}

Figure 9. Formation of new valence and conduction bands by electron donor and acceptor atoms, enabling a UV-active material to respond to visible light.

It wasn't until Kudo et al began doping the perovskite $\mathrm{SrTiO}_{3}$ with metals such as $\mathrm{Cr}, \mathrm{Ru}, \mathrm{Ir}, \mathrm{Mn}, \mathrm{Sb}, \mathrm{Ta}, \mathrm{Ni}$, and $\mathrm{Rh}$, that quantum efficiencies started to increase ${ }^{123}$. Rh (1\%) doped strontium titanate demonstrated a quantum efficiency of $5.2 \%$ for hydrogen production at $420 \mathrm{~nm}$, the highest value among the different dopants. Rhodium forms an electron donor level more negative than that of oxygen $2 p$ orbitals, narrowing the light absorption into the visible range. However, oxygen production was nearly inhibited, presumably due to $\mathrm{Rh}_{\mathrm{x}} \mathrm{O}_{\mathrm{y}}$ surface species blocking certain active sites. The unique redox functionality of $\mathrm{Rh}^{3+/ 4+}$ is thought to play a part in the enhanced activity. Rhodium can also be doped into other perovskites such as $\mathrm{CaTiO}_{3}$, and $\mathrm{Ca}_{3} \mathrm{Ti}_{2} \mathrm{O}_{7}$, however due to the complex band structure, 
the Rh dopant was indifferent, and efficiencies were immeasurably small ${ }^{124,125}$.

Wolframite tantalates also showed activity when doped with various metals. Zou et al reported that nickel doped $\mathrm{InTaO}_{4}$ can split water in stoichiometric amounts without any electron donor/acceptor with a QY of $0.66 \%(402 \mathrm{~nm})^{126}$. However, it must be in the presence of a $\mathrm{RuO}_{2}$ or $\mathrm{NiO}$ cocatalyst. Doped $\mathrm{NiO}-\mathrm{InTaO}_{4}: \mathrm{Ni}$ has also been investigated and found to be less active due to having a larger band gap (2.6 eV undoped, $2.3 \mathrm{eV}$ doped). This is because the $\mathrm{In}^{2+}$ ions are dislodged by $\mathrm{Ni}^{2+}$, which consequently causes the lattice to contract, and in turn reduces the band gap by the introduction of partially filled nickel $3 \mathrm{~d}$ orbitals. $\mathrm{NaBO}_{3}$, a series of known UV-active materials, were investigated by Kudo et al $(\mathrm{B}=\mathrm{Nb}$ or $\mathrm{Ta})^{127} \cdot \mathrm{NaNbO}_{3}$ doped with iridium and either $\mathrm{Sr}, \mathrm{Ba}$, or La shows activity for separate hydrogen and oxygen evolution. On the other hand $\mathrm{NaTaO}_{3}$ doped with iridium and $\mathrm{Sr} / \mathrm{Ba} / \mathrm{La}$ only showed activity for hydrogen evolution due to the change in band structure and blocking of oxygen evolution sites.

Due to the nature of a dopant, i.e. the incorporation into the lattice of a foreign element, the number of electronic states is inherently low. Therefore, upon excitation, the new band in the otherwise forbidden region only can provide a limited amount of photoexcited electrons to the conduction band for reduction reactions. The same amount of limited holes are left behind for potential oxidation. In other words, the contribution to the density of states by this dopant is small in comparison to that of the bulk band structure, and therefore the maximum efficiency will also be low. Furthermore, one cannot simply increase dopant amount to accordingly increase states, otherwise crystal structure will slowly change/buckle, and original band structure will deteriorate. Ideally therefore, logic would dictate to focus research on the discovery of new pure phase materials, or by enhancing suitable pre-existing compounds.

One group used an ingenious way of circumventing crystal structure deformation by creating a solid solution, whereby two wide band gap semiconductors create one narrow gap solid solution; GaN:ZnO (Figure 10). Domen et al produced a range of visible light nitrides/oxynitrides ${ }^{128,129,130,131}$. The nitrides/oxynitrides need scavenger species to function, and also need the $\mathrm{pH}$ to be adjusted by a lanthanum containing material. Systems comprising of solid solutions of oxides and nitrides have been reported with some positive results ${ }^{18,132,133}$. $\mathrm{GaN}: \mathrm{ZnO}\left(\mathrm{Ga}_{0.88} \mathrm{~N}_{0.88} \mathrm{Zn}_{0.12} \mathrm{O}_{0.12}\right)$, is able to split water with a high QY under visible illumination without scavenger species 
$(\mathrm{QY}=5.9 \%, 420 \mathrm{~nm})$. In order to obtain this record high QY for overall water splitting, the $\mathrm{pH}$ must be adjusted to 4.5 (using $\mathrm{H}_{2} \mathrm{SO}_{4}$ ), and the gallium-zinc composite must be coated with $\mathrm{Rh}_{2-\mathrm{x}} \mathrm{Cr}_{\mathrm{x}} \mathrm{O}_{3}$ cocatalyst nanoparticles (10-30 $\mathrm{nm}$ diameter). If the cocatalyst isn't present, no water splitting is observed. Both GaN and $\mathrm{ZnO}$ have an almost identical crystal structure, including space group and lattice parameters, and therefore the crystal structure is not deformed when a solid solution is formed.

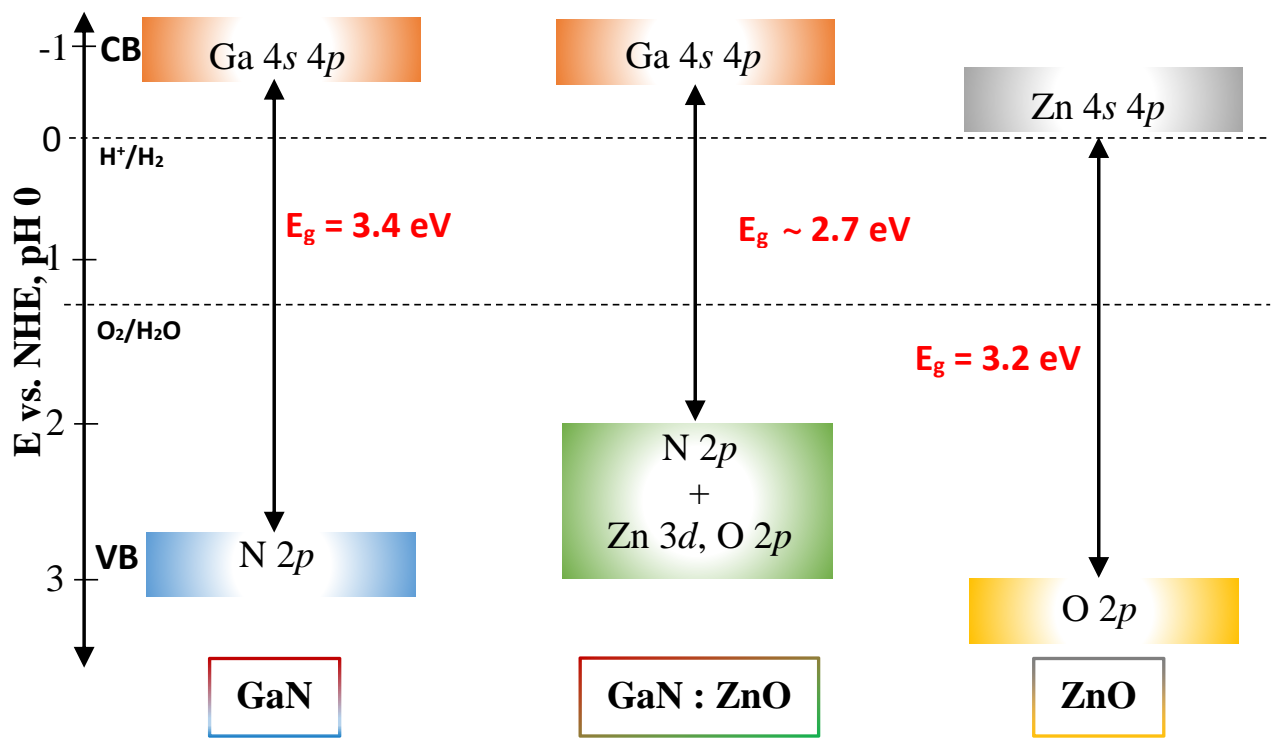

Figure 10. Illustration of GaN: $\mathrm{ZnO}$ solid solution. Conduction band orbitals remain as Ga $4 \mathrm{~s} 4 \mathrm{p}$, whilst valence band is hybridised to N2p, Zn3d and O2p. Adapted from Maeda et $\mathrm{al}^{132}$.

In terms of native oxide photocatalysts, $\mathrm{WO}_{3}$ is well-documented for oxygen production in water in the presence of an electron scavenger such as $\mathbf{A g}^{+}$; however the low conduction band prevents hydrogen production. Attempts of incorporating different elements into tungsten oxide have been successful, and hydrogen production is a possibility by creating new LUMO and HOMO levels. $\mathrm{AgInW}_{2} \mathrm{O}_{8}, \mathrm{AgBiW}_{2} \mathrm{O}_{8}$ and $\mathrm{Ag}_{2} \mathrm{WO}_{4}$ have been experimentally proven to produce both $\mathrm{O}_{2}$ and $\mathrm{H}_{2}$, albeit in relatively low yields ${ }^{134,135}$. 
A large amount of reports have been published in the last 15 years or so on $\mathrm{BiVO}_{4}$ (monoclinic crystal structure), some with quantum yields of up to $9 \%$ for oxygen production under visible light $(450 \mathrm{~nm})$ irradiation ${ }^{17,136}, 31$. It was found that the activity of $\mathrm{BiVO}_{4}$ is heavily dependent on the preparation method and crystal phase - in particular, the monoclinic phase is principally the most active. The efficiency has also been shown to be irrespective of surface area and more influenced by defect concentration; high temperature solid state reactions yield low efficiencies, and wet chemical methods promote higher yields due to phase purity. $\mathrm{BiVO}_{4}$ cannot in fact reduce water to produce hydrogen due to its low conduction band (thought to be between 0.1 and $0.7 \mathrm{eV}$ vs NHE). Another monoclinic material, $\operatorname{In}_{\mathrm{x}} \mathrm{MTi}_{\mathrm{y}} \mathrm{O}_{\mathrm{z}}(\mathrm{M}=\mathrm{Ni}, \mathrm{Cr}$, or both) was investigated, yielding results which suggest that hybridised electron orbitals lead to a higher charge carrier transportation.

Sulphides have potential as visible light photocatalysts for the production of solar hydrogen; but their application has been limited due to photocorrosion. CdS has been stringently investigated due to its narrow band gap and ideal band positions for both $\mathrm{O}_{2}$ and $\mathrm{H}_{2}$ evolution. Two groups studied CdS, reporting hydrogen production using edetic acid as an electron donor ${ }^{137,138}$. They found that with the addition of $\mathrm{Pt}$ cocatalyst, $\mathrm{H}_{2}$ evolution rates will increase by a factor of 10 . By introducing $\mathrm{Na}_{2} \mathrm{SO}_{3}$ into the solution a quantum yield of $35 \%$ (at $430 \mathrm{~nm}$ ) can be achieved. Progressing from this, if CdS is loaded with a cocatalyst comprising of Pt-PdS, a quantum yield of $93 \%$ can be achieved for photocatalytic hydrogen production ${ }^{139}$. Unique to sulphide photocatalysts, another sulphide $\left(\mathrm{Rh}_{2} \mathrm{~S}_{3}, \mathrm{Ru}_{2} \mathrm{~S}_{3}, \mathrm{MoS}_{2}\right)$ can be added to $\mathrm{CdS}$ or $\mathrm{CuGa}_{3} \mathrm{~S}_{5}$ as catalysts, and perform better than their metallic counterparts ${ }^{140,141,142}$. The activity increase is attributed to a better coupling between structures, i.e. the presence of sulphur in the catalyst enables a strong metal support interaction between substrate and metal. This in turn increases electron-hole separation and consequently more probable reduction reactions. In an alternate report, Gratzel's group have shown that loading $\mathrm{CdS}$ with $\mathrm{Pt}$ and $\mathrm{RuO}_{2}$ leads to a decrease in photocorrosion $^{143}$. In order to increase the resistance to photocorrosion, electron donors such as $\mathrm{S}^{2-}, \mathrm{SO}_{3}{ }^{2-}$ must be introduced into the solution. Various strategies have been employed to overcome such problems as; encapsulation of CdS nanoparticles ${ }^{144}$, formation of CdS composites with $\mathrm{TiO}_{2}{ }^{145}$ and $\mathrm{ZnS}^{146}$, and doping of $\mathrm{CdS}$ by noble metals and transition metals $^{147}$ but with limited success. 
A recent report from Ye et al. have found that a phosphate compound could function as a photocatalyst with a record-breaking quantum yield ${ }^{7}$. Silver orthophosphate $\left(\mathrm{Ag}_{3} \mathrm{PO}_{4}\right)$ has a maximum quantum yield for $\mathrm{O}_{2}$ evolution of $89 \%$ at $420 \mathrm{~nm}$, yet is only $50 \%$ at $500 \mathrm{~nm}$. However, the orthophosphate compound does require the use of an electron scavenger to inhibit self-decomposition. The band gap was measured by UV-Vis to be approximately $2.4 \mathrm{eV}$. The conduction band is more positive than $0 \mathrm{~V}$ vs NHE, and thus the compound cannot produce hydrogen from water. The valence bands are made up of hybridised silver $4 \mathrm{~d}$ and oxygen $2 \mathrm{p}$ orbitals, while the conduction band is composed mainly of hybridised silver $5 \mathrm{~s} 5 \mathrm{p}$, and a small amount of phosphorus 3s electron orbitals ${ }^{7}$. These highly dispersive conduction bands are thought to provide an efficient charge transfer system with low charge recombination, and result in the compound's high photooxidation. A computational study by Umezawa et al used DFT to ascertain the exact mechanisms behind the remarkable activity ${ }^{148}$. They found that the formation of strong $\mathrm{P}-\mathrm{O}$ bonds in $\mathrm{PO}_{4}$ tetrahedra deteriorates the covalent nature of Ag-O bonds. This decreases the hybridisation between $\mathrm{Ag} \mathrm{d}$ and $\mathrm{O} \mathrm{p}$ bands, and as a consequence, excludes the $\mathrm{d}$ character from the conduction band minimum. This then leaves dual $\mathrm{Ag} \mathrm{s}-\mathrm{Ag} \mathrm{s}$ hybrid states, with the absence of localised $\mathrm{d}$ character. As an interesting side point, the DFT results highlight the isotropic nature of the electrons in $\mathrm{Ag}_{3} \mathrm{PO}_{4}$; the mass of electrons $\left(\mathrm{m}_{\mathrm{e}}{ }^{*}\right)$ is small in all directions. This is the opposite of holes, whereby the mass is anisotropic in three different primary directions; $<\mathbf{1 0 0}>,\langle\mathbf{1 1 0}>$, and $<\mathbf{1 1 1}\rangle$ as later reported.

Currently, no efforts have been made in order to stabilise the compound, and little follow up literature on water photooxidation is available. Bi et al did however test different exposing facets for the photodegradation of organic dyes (methyl orange -'MO', methylene blue - 'MB', and Rhodamine B - 'RhB'), for which there was a difference in degradation rate depending on what facet was predominant $(\{100\}$ - cubic, or $\{110\}-$ rhombododecahedron $)^{149,150}$. According to the reports, the two facets tested showed considerably higher activity for organic degradation than $\mathrm{N}^{-\mathrm{TiO}_{2}}$ and multifaceted (roughly spherical, and therefore a mixture of all facets) $\mathrm{Ag}_{3} \mathrm{PO}_{4}$. The group continued to mention that although the enhanced activity did not correlate with surface area, that is a higher surface area did not give a higher activity, a connection between the DFT calculated surface energy and activity is evident. As the surface energy increases, so too does the degradation rate of organic contaminants. A higher energy 
surface facet (such as the low index $\{100\}$ or $\{110\}$ planes) is inherently more 'unstable', and therefore possesses a rougher or more 'kinked' surface. From calculations of $\mathrm{Ag}_{3} \mathrm{PO}_{4}$, it can be concluded that a higher number of oxygen vacancies create more active sites on the surface to which the organic molecule can adsorb - thereby enabling the degradation reaction to occur faster. However since the energetic requirements of organic degradation are different than that of water splitting, it was not clear if the 'facet effect' would be beneficial to water splitting in this particular case.

In 2008, an organic-polymer-based photocatalyst, graphitic carbon nitride $\left(\mathrm{g}-\mathrm{C}_{3} \mathrm{~N}_{4}\right)$, was found to show sufficient redox power to dissociate water under visible light in a suspension system ${ }^{151}$. The compound was fabricated by simply calcining cyanamide in either air, or a nitrogen atmosphere. Historically, the structure of carbon nitride has been debated since initial reports in 1834; with melon, melem and melam structures all being proposed $^{152,153,154}$. It is now accepted that the graphitic form of carbon nitride, which is photocatalytically active, is initially composed of stable C$\mathrm{N}$ heptazine (tri-s-triazine) sheets, hydrogen bonded in a zig-zag formation ${ }^{155}$. Upon further condensation and removal of $\mathrm{NH}_{3}$ groups, heptazine units continue to polymerise to form what is now conventionally known as graphitic carbon nitride (Figure 11) ${ }^{156,157}$. The first experimental example of $\mathrm{g}-\mathrm{C}_{3} \mathrm{~N}_{4}$ showed that the compound could evolve hydrogen with and without a cocatalyst. However, the presence of photodeposited platinum increased yields more than 2.5 times. DFT calculations confirmed UV-vis results that the band gap was approximately $2.7 \mathrm{eV}$. Further calculations revealed that the conduction band is made up of carbon $p_{z}$ orbitals, whilst the valence band is primarily composed of nitrogen $p_{z}$ orbitals. Both LUMO and HOMO are contained within the heptazine monomer and no charge is transferred between heptazine units. Tertiary nitrogen merely links heptazine units together, however no reports to date have shown an isolated heptazine unit to itself be a functional photocatalyst - meaning that the role of linking nitrogen atoms could be crucial to the activity of $\mathrm{g}-\mathrm{C}_{3} \mathrm{~N}_{4}$.

Due to the stability and elemental abundance, intensive research efforts have been made in order to potentially increase the efficiency. Research efforts by Dong et al have shown that polymeric carbon nitride can be fabricated not just from cyanamide, but derivatives such as dicyandiamide (DCDA), thiourea, and urea. ${ }^{158,159}$ The latest documented quantum yields under visible light for $\mathrm{H}_{2}$ production from water using $\mathrm{g}-\mathrm{C}_{3} \mathrm{~N}_{4}$ however, do not exceed $4 \%$ (excluding dye-sensitized systems) ${ }^{151,160,161,162,163,164,165,166}$, which 
is far from the requirements demanded by researchers ${ }^{66}$. Researchers have also made modifications to $\mathrm{g}-\mathrm{C}_{3} \mathrm{~N}_{4}$ based photocatalysts, e.g. via nanocasting ${ }^{165}$, soft-templating ${ }^{167}$ and chemical functionalization, i.e. by post $^{168}$ or in situ heteroatom doping ${ }^{167}$. Despite the various methods used to increase efficiency, the highest quantum efficiency remains at $3.75 \%$ for hydrogen evoltuion under visible light $(420 \mathrm{~nm})^{165}$.
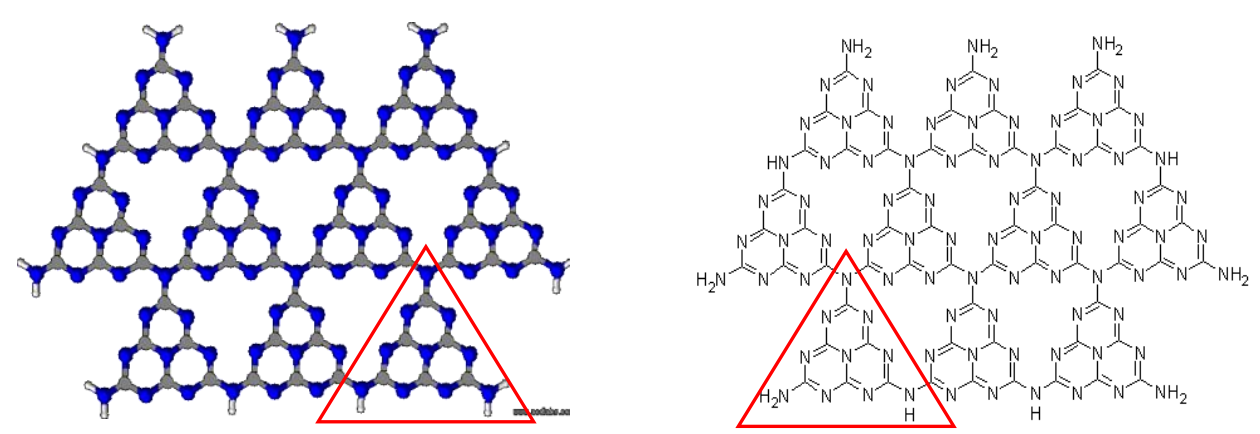

Heptazine unit

$\mathbf{C}_{\mathrm{x}} \mathbf{N}_{\mathbf{y}} \mathbf{H}_{\mathrm{z}}$

Figure 11. Ball \& stick (left), and molecular schematic drawing of $g-\mathrm{C}_{3} \mathrm{~N}_{4}$ (right), using ChemSketch ${ }^{\odot}$. A single heptazine unit is highlighted in red. Graphitic carbon nitride is composed of; carbon (grey), nitrogen (blue), and hydrogen atoms (white).

The first of two ways to achieve colloidal overall water splitting under visible light is to use a single photocatalyst; the standout (and only working example) is GaN: $\mathrm{ZnO}$ (with Rh-Cr catalyst), achieving a quantum yield of $5.9 \%$ at a working wavelength range of $420-440 \mathrm{~nm}^{132}$. The other is nickel doped $\mathrm{InTaO}_{4}\left(\mathrm{NiO}_{\mathrm{x}}\right.$ catalyst), which has demonstrated a quantum yield of $0.66 \%$ at $402 \mathrm{~nm}^{126}$. The main issue with the two aforementioned systems lies with the exclusivity, that is, it is difficult to systematically build upon the existing research because of the specialised nature of the compounds. For example, gallium nitride and zinc oxide are an almost perfect match with one another in terms of crystal structure. That coupled with the fortuitous band structure yields a photocatalyst system which is unique.

The second approach to visible light water splitting is to use a two photon process, termed a 'Z-Scheme'. Inspired by natural photosynthesis, Bard 
proposed a biomimetic analogy composed of two inorganic semiconductor photocatalysts in 1979 and recently there have been some successes in the field based on various different photocatalysts. ${ }^{169,170}$.

\subsubsection{Z-Scheme systems}

Nature splits water into $\mathrm{O}_{2}$ and the equivalent $\mathrm{H}_{2}$ species by a double excitation process in PSII ('Photo-System') and PSI, instead of a single excitation, in which the two half reactions are spatially separated and take place in PSII and PSI, Figure 12. This overcomes the main problems of a singular photocatalytic water splitting system both kinetically and thermodynamically, as well described in a previous review ${ }^{171}$. In short the Z-scheme has the potential to be more practical than a single photocatalyst $^{172}$.

Briefly, a Z-scheme system is a combination of two semiconductor photocatalysts, in which each photocatalyst is responsible for one half reaction and a soluble mediator helps electron transfer between the two photocatalysts so that an ideal cycle can be completed. The mediator is very important because it dramatically inhibits the fast unfavourable recombination of charge, analogous to the electron transport chain between PSII and PSI. Given such advantages of a double excitation process, there are many researchers working on either two photocatalysts each of which favours either $\mathrm{H}_{2}$ or $\mathrm{O}_{2}$ production, or a new mediator to efficiently transfer charge between two photocatalysts. 

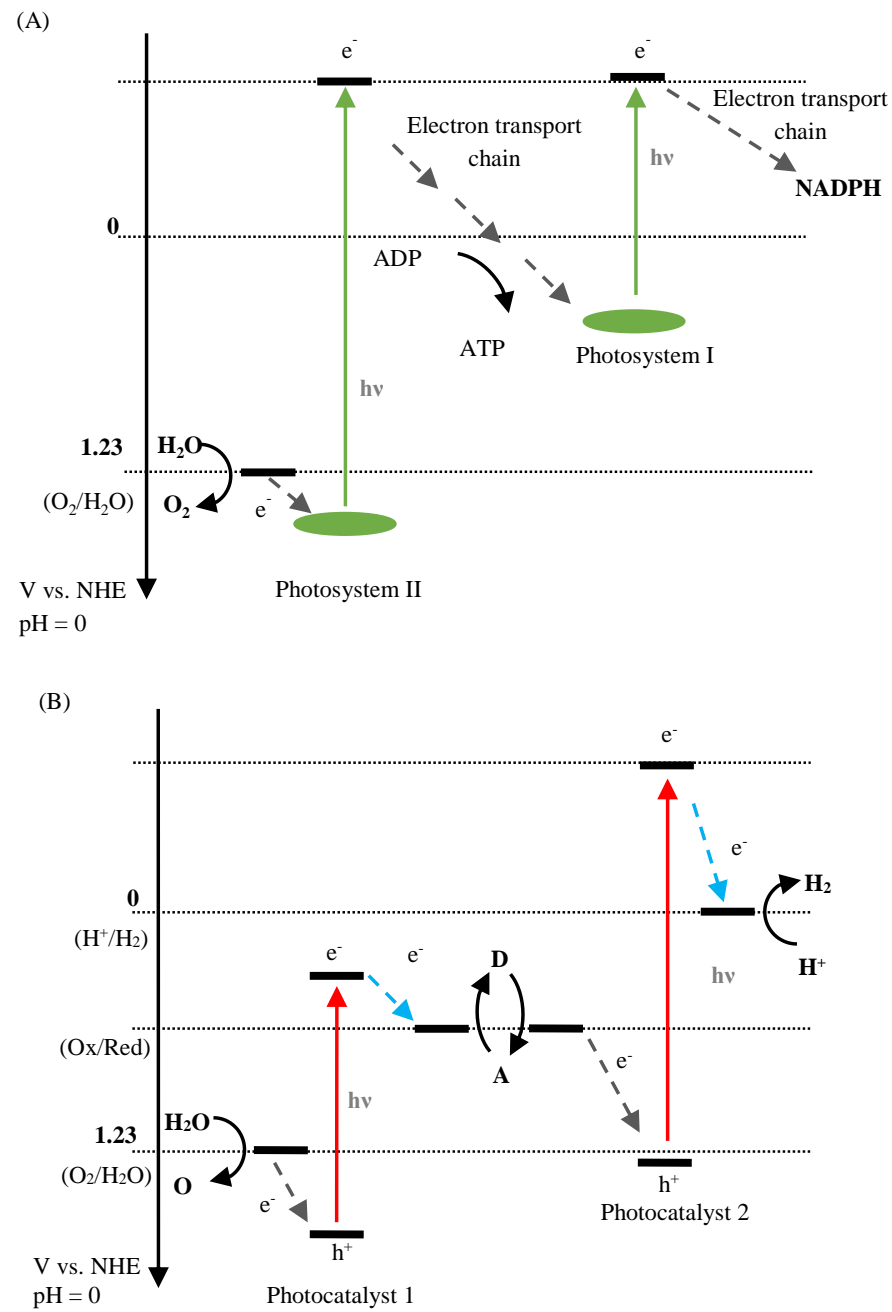

Figure 12. (A) Natural photosynthesis (B) An artificial analogy involving two different photocatalysts possessing two different band positions ( $\mathrm{A}$ is electron acceptor, $\mathrm{D}$ is electron donor $)^{172}$.

Initially, Darwent and Mills showed that the reduction potential of $\mathrm{Fe}^{3+}$ (from $\mathrm{FeCl}_{3}$ ) was near the conduction band of $\mathrm{WO}_{3}$, i.e. the iron ion acted as an electron scavenger and thus $\mathrm{WO}_{3}$ could produce oxygen under visible light from water ${ }^{173}$. This observation was also confirmed by Graetzel ${ }^{12}$. In 2001, Sayama collaborated with Abe and created a UV-responsive anatase $\mathrm{TiO}_{2}$ - rutile $\mathrm{TiO}_{2}$ dual photocatalyst z-scheme system ${ }^{174}$, using an iodide (redox couple: $\mathrm{I}^{-} / \mathrm{IO}_{3}{ }^{-}$, from $\mathrm{NaI}$ ) mediator inspired by the early works of Kim et $\mathrm{al}^{175}$. This mediator was also used in a visible light system based on 
Pt-loaded mixed Cr/Ta doped $\mathrm{SrTiO}_{3}$, and $\mathrm{PtO}_{\mathrm{x}}-\mathrm{WO}_{3}$, which can achieve a quantum yield of $1 \%$ for overall water splitting ${ }^{176}$. In a water splitting reaction, the redox couples behave as follows:

Equation 29. $\mathrm{Fe}^{2+/ 3+}$ redox potentials

$$
\begin{gathered}
O_{2} \text { photocatalyst: } \mathrm{Fe}^{3+}+\mathrm{e}^{-} \rightarrow \mathrm{Fe}^{2+} \quad(0.771 \mathrm{~V} \text { vs NHE }) \\
2 \mathrm{H}_{2} \mathrm{O}+4 \mathrm{~h}^{+} \rightarrow \mathrm{O}_{2}+4 \mathrm{H}^{+} \\
\mathrm{H}_{2} \text { photocatalyst }: \quad \mathrm{Fe}^{2+}+h^{+} \rightarrow \mathrm{Fe}^{3+} \\
4 \mathrm{H}^{+}+4 \mathrm{e}^{-} \rightarrow 2 \mathrm{H}_{2}
\end{gathered}
$$

Equation 30. $\mathrm{I}^{-} / \mathrm{IO}_{3}{ }^{-}$redox potentials

$$
\begin{gathered}
\mathrm{H}_{2} \text { photocatalyst: } \mathrm{I}^{-}+3 \mathrm{H}_{2} \mathrm{O}+6 \mathrm{~h}^{+} \rightarrow \mathrm{IO}_{3}^{-}+6 \mathrm{H}^{+}(0.71 \mathrm{~V} \text { vs NHE }) \\
6 \mathrm{H}^{+}+6 e^{-} \rightarrow 3 \mathrm{H}_{2} \\
\mathrm{O}_{2} \text { photocatalyst: } \mathrm{IO}_{3}^{-}+6 e^{-}+3 \mathrm{H}_{2} \mathrm{O} \rightarrow \mathrm{I}^{-}+6 \mathrm{OH}^{-} \\
2 \mathrm{H}_{2} \mathrm{O}+4 \mathrm{~h}^{+} \rightarrow \mathrm{O}_{2}+4 \mathrm{H}^{+}
\end{gathered}
$$

The activity of rutile $\mathrm{TiO}_{2}$ and $\mathrm{WO}_{3}$, according to reports, stems from their suitable surface and band structure for the reduction of $\mathrm{Fe}^{3+}$ and $\mathrm{IO}_{3}{ }^{-}$, and more importantly, the selective oxidation of water, even in the presence of electron donors such as $\mathrm{Fe}^{2+}$ and $\mathrm{I}^{-177,178}$. Therefore, these are considered optimum water oxidation photocatalysts in Z-Schemes. In 2004, $\mathrm{BiVO}_{4}$ was also shown to function as an oxygen evolution photocatalyst in the presence of the $\mathrm{Fe}^{2+/ 3+}$ mediator, when paired with $\mathrm{Pt}$ loaded $\mathrm{SrTiO}_{3}$ doped with $\mathrm{Rh}^{170}$. As mentioned previously $\mathrm{BiVO}_{4}$ has a bandgap of approximately 2.4 $\mathrm{eV}$, and therefore can absorb visible light up to $520 \mathrm{~nm}$ in most cases. In theory, a semiconductor with a smaller band gap can absorb more light, and potentially achieve higher efficiencies. However, researchers have shown that even by using an $\mathrm{O}_{2}$ photocatalyst such as $\mathrm{BiVO}_{4}$, which has a smaller band gap than either $\mathrm{Bi}_{2} \mathrm{MoO}_{6}$ or $\mathrm{WO}_{3}$, the system is still limited in terms of efficiency by a $\mathrm{H}_{2}$ photocatalyst $\mathrm{Pt} / \mathrm{SrTiO}_{3}: \mathrm{Rh}$. Therefore, it would be advantageous to find a more efficient hydrogen evolving photocatalyst for use in a Z-scheme water splitting system. Further to this, another drawback of the 'Z-scheme' process are notably back reactions, and 
side reactions, both of which hinder efficiencies (the most common of which is where $I^{-}$is oxidised to $I_{3}^{-}$, which then cannot be reduced easily as it isn't an efficient electron acceptor.).

Various other photocatalysts can be used as a hydrogen evolution photocatalyst in a Z-scheme water splitting system, most notably nitrides and oxynitrides ${ }^{179}$, as seen in Table 1. Tantalum oxynitride (TaON) has been shown to oxidise $\mathrm{I}^{-}$to $\mathrm{IO}_{3}^{-}$effectively, and when coupled with either $\mathrm{WO}_{3}$ or $\mathrm{Ta}_{2} \mathrm{~N}_{5}$, can participate in a $\mathrm{Z}$-scheme system, with quantum yields up to $6.3 \%$ at $420 \mathrm{~nm}$ - the highest reported to date ${ }^{179,180}$. The authors claim that despite the high efficiency, the rate limiting step originally lies with the hydrogen production photocatalyst $(\mathrm{TaON})$ - to which they added a protection layer of $\mathrm{ZrO}_{2}$, helping to increase the thermal and chemical stability. As TaON is fabricated by nitridation of $\mathrm{Ta}_{2} \mathrm{O}_{5}$, and thus is an "intermediate phase" between $\mathrm{Ta}_{2} \mathrm{O}_{5}$ and $\mathrm{Ta}_{3} \mathrm{~N}_{5}$, the crystallinity of the material is poor and results in many surface defects ${ }^{181}$. The authors then modified TaON with $\mathrm{ZrO}_{2}$ in situ to maintain the $\mathrm{Ta}^{5+}$ cation at the surface (make Ta more 'cationic'), and to prevent the formation of lower valences, such as $\mathbf{T a}^{3+}$ or $\mathbf{T a}^{4+}$ which act as electron recombination centres and hinder efficiency. Despite tantalum's high cost, the system illustrates that nitrides and oxynitrides can be used in Z-scheme water splitting systems, and more importantly, due to the positioning of the redox potentials of both $\mathrm{I}^{-} / \mathrm{IO}_{3}$ and $\mathrm{Fe}^{2+} / \mathrm{Fe}^{3+}$, be stable in both reduction and oxidation.

To briefly conclude, the latest studies show that quantum yields are relatively low for overall water splitting in the presence of a redox mediator, are no larger than $6 \%$ under visible light, as seen in Table 1. However, many photocatalysts can potentially be used, as there is some flexibility with both band position and electron mediator. This bodes very well for future research, as the process is not as specialised as the aforementioned single photocatalyst water splitting system. 
Table 1. Summary of water splitting systems using a Z-scheme setup, currently reported in the literature ${ }^{181}$.

\begin{tabular}{|c|c|c|c|c|c|}
\hline \multicolumn{3}{|c|}{ Photocatalyst (available wavelength) } & \multirow[b]{2}{*}{ Redox mediator } & \multirow[b]{2}{*}{ Maximum apparent quantum yield (\%) } & \multirow[b]{2}{*}{ Reference } \\
\hline Entry & $\mathbf{H}_{2}$ & $\mathbf{O}_{2}$ & & & \\
\hline 1 & $\mathrm{Fe}^{2+}(<280 \mathrm{~nm})$ & $\mathrm{RuO}_{2} / \mathrm{WO}_{3}(<460 \mathrm{~nm})$ & $\mathbf{F e}^{3+} / \mathbf{F e}^{2+}$ & No available information & 182 \\
\hline 2 & $\mathrm{Pt} / \mathrm{TiO}_{2}$ anatase $(<380 \mathrm{~nm})$ & $\mathrm{TiO}_{2}$ rutile $(<410 \mathrm{~nm})$ & $\mathrm{IO}_{3}^{-} / \mathrm{I}^{-}$ & $1(350 \mathrm{~nm})$ & 174 \\
\hline 3 & $\mathrm{Pt} / \mathrm{SrTiO}_{3}: \mathrm{Cr} / \mathrm{Ta}(<550 \mathrm{~nm})$ & $\mathrm{PtO}_{\mathrm{x}} / \mathrm{WO}_{3}(<460 \mathrm{~nm})$ & $\mathrm{IO}_{3}{ }^{-} / \mathrm{I}^{-}$ & $1(420 \mathrm{~nm})$ & 176 \\
\hline 4 & $\mathrm{Pt} / \mathrm{SrTiO}_{3}: \mathrm{Bi} / \mathrm{Ga}(<370 \mathrm{~nm})$ & $\mathrm{SrTiO}_{3}: \mathrm{In} / \mathrm{V}(<370 \mathrm{~nm})$ & $\mathrm{IO}_{3}^{-} / \mathrm{I}^{-}$ & No available information & 183 \\
\hline 5 & $\mathrm{Pt} / \mathrm{SrTiO}_{3}: \mathrm{Rh}(<520 \mathrm{~nm})$ & $\begin{array}{l}\mathrm{Ru} / \mathrm{SrTiO}_{3}: \mathrm{In} / \mathrm{V}(< \\
520 \mathrm{~nm})\end{array}$ & $\mathrm{IO}_{3}^{-} / \mathrm{I}^{-}$ & $0.33(360 \mathrm{~nm}) 0.056(420 \mathrm{~nm}) 0.039(480 \mathrm{~nm})$ & 184 \\
\hline 6 & $\mathrm{Pt} / \mathrm{TiO}_{2}$ anatase $(<400 \mathrm{~nm})$ & $\mathrm{NO}_{3}^{-}(<250 \mathrm{~nm})$ & $\mathrm{NO}_{3}^{-} / \mathrm{NO}_{2}{ }^{-}$ & no data & 185 \\
\hline 7 & $\mathrm{Pt} / \mathrm{SrTiO}_{3}: \mathrm{Rh}(<520 \mathrm{~nm})$ & $\mathrm{BiVO}_{4}(<520 \mathrm{~nm})$ & $\mathrm{Fe}^{3+} / \mathrm{Fe}^{2+}$ & $0.4(420 \mathrm{~nm}), 0.3(440 \mathrm{~nm})$ & 170 \\
\hline 8 & $\mathrm{Pt} / \mathrm{SrTiO}_{3}: \mathrm{Rh}(<520 \mathrm{~nm})$ & $\mathrm{Bi}_{2} \mathrm{MoO}_{6}(<450 \mathrm{~nm})$ & $\mathrm{Fe}^{3+} / \mathrm{Fe}^{2+}$ & $0.2(440 \mathrm{~nm})$ & 170 \\
\hline 9 & $\mathrm{Pt} / \mathrm{SrTiO}_{3}: \mathrm{Rh}(<520 \mathrm{~nm})$ & $\mathrm{WO}_{3}(<460 \mathrm{~nm})$ & $\mathrm{Fe}^{3+} / \mathrm{Fe}^{2+}$ & $0.5(420 \mathrm{~nm}), 0.2(440 \mathrm{~nm})$ & 170 \\
\hline 10 & $\mathrm{Ru} / \mathrm{SrTiO}_{3}: \mathrm{Rh}(<520 \mathrm{~nm})$ & $\mathrm{BiVO}_{4}(<520 \mathrm{~nm})$ & $\mathrm{Fe}^{3+} / \mathrm{Fe}^{2+}$ & $0.3(420 \mathrm{~nm})$ & 186 \\
\hline 11 & $\mathrm{Ru} / \mathrm{SrTiO}_{3}: \mathrm{Rh}(<520 \mathrm{~nm})$ & $\mathrm{BiVO}_{4}(<520 \mathrm{~nm})$ & none & $1.7(420 \mathrm{~nm})$ & 187 \\
\hline 12 & $\mathrm{Ru} / \mathrm{SrTiO}_{3}: \mathrm{Rh}(<520 \mathrm{~nm})$ & $\mathrm{BiVO}_{4}(<520 \mathrm{~nm})$ & $\begin{array}{l}\text { Solid - reduced } \\
\text { graphene oxide }\end{array}$ & $1.03(420 \mathrm{~nm})$ & 188 \\
\hline 13 & $\mathrm{Pt} / \mathrm{TaON}(<520 \mathrm{~nm})$ & $\mathrm{PtO}_{\mathrm{x}} / \mathrm{WO}_{3}(<460 \mathrm{~nm})$ & $\mathrm{IO}_{3}^{-} / \mathrm{I}^{-}$ & $0.5(420 \mathrm{~nm})$ & 189,190 \\
\hline 14 & $\mathrm{Pt} / \mathrm{TaON}(<520 \mathrm{~nm})$ & $\mathrm{RuO}_{2} / \mathrm{TaON}(<520 \mathrm{~nm})$ & $\mathrm{IO}_{3}^{-} / \mathrm{I}^{-}$ & $0.1(420-440 \mathrm{~nm})$ & 191 \\
\hline 15 & $\mathrm{Pt} / \mathrm{ZrO}_{2} / \mathrm{TaON}(<520 \mathrm{~nm})$ & $\mathrm{PtO}_{\mathrm{x}} / \mathrm{WO}_{3}(<460 \mathrm{~nm})$ & $\mathrm{IO}_{3}^{-} / \mathrm{I}^{-}$ & $6.3(420 \mathrm{~nm})$ & 180,192 \\
\hline 16 & $\mathrm{Pt} / \mathrm{ZrO}_{2} / \mathrm{TaON}(<520 \mathrm{~nm})$ & $\mathrm{Ir} / \mathrm{TiO}_{2} / \mathrm{Ta}_{3} \mathrm{~N}_{5}(<600 \mathrm{~nm})$ & $\mathrm{IO}_{3}^{-} / \mathrm{I}^{-}$ & No available information & 179 \\
\hline 17 & $\mathrm{Pt} / \mathrm{ZrO}_{2} / \mathrm{TaON}(<520 \mathrm{~nm})$ & $\mathrm{RuO}_{2} / \mathrm{TaON}(<520 \mathrm{~nm})$ & $\mathrm{IO}_{3}^{-} / \mathrm{I}^{-}$ & No available information & 193 \\
\hline
\end{tabular}


2.3.4 Effect of morphology, crystallinity and size on the activity of photocatalysts

In sections 2.3.1 and 2.3.2 it was noted that both band structure and magnitude could contribute to the performance of a photocatalyst, however, this is one of just many factors which come into play. Cocatalyst choice, surface area, number of defects, among others all play vital parts in determining efficiency. An important point which was touched on in section 2.1.4 was the morphology of particles. The three step process mentioned in Figure 5 illustrates the importance of surface area and crystallinity. It is noted that the increase in crystallinity leads to a decrease in defects - which in turn decreases the recombination probability of charge carriers in the bulk and on the surface. On the other hand, high temperature calcinations which often result in good crystallinity, suffer from low surface area, and thus low number of surface active sites.

If a particle is of small size, on the nanoscale $\left(10^{-9} \mathrm{~m}\right)$, then the distance the charge carrier (electron or hole) has to travel to reach the surface is decreased. However, decreasing a particles' size leads to a quantum sizing effect, effectively increasing the band gap ${ }^{194,195}$. This arises when charge carriers are constrained to a specific portion of space by a potential barrier; the region is less than or equal to their de Broglie wavelength, and the energy states associated with the particle become quantized, as opposed to continuous. It can be difficult to achieve particles with high crystallinity, and correct size. Several methods are available which are used to fabricate highly crystalline nanoscale materials, including hydrothermal synthesis (both microwave assisted and conventional) and wet chemical techniques (e.g. sol-gel method, phase transfer, ion exchange). These techniques are often deployed depending on what temperature the reaction between two or more reactants happen. Dropping below room temperature for a certain reaction would yield smaller particles and reduce agglomeration/grain growth, however, for another; there would not be enough thermal energy to facilitate a reaction. Conversely, if a reaction happens at temperature $\mathrm{T}$ and pressure $P$, then exceeding these parameters will only result in the accumulation of particles. Microwave assisted hydrothermal synthesis is one of the most prominent methods of nanocrystal synthesis. Its advantages over conventional heating include; direct control over temperature and pressure within a sealed vessel, activation of rotational and vibrational nodes within molecules, high interaction with polar molecules and uniform 
heating. These all lead to an overall quicker reaction time, higher product yield and the use of much lower temperatures ${ }^{196,197}$. Conventional hydrothermal synthesis involves simply heating a vessel in an oven or oil bath; the vessel is sealed and high pressures build up inside. Wang et al. found that by varying temperature they were able to selectively produce different sized nanocrystals ${ }^{198}$. Higher temperatures yield larger particles; $\mathrm{Ag}$ samples reacted at $90{ }^{\circ} \mathrm{C}$ resulted in $6 \mathrm{~nm}$ particles, whilst at a temperature of $180{ }^{\circ} \mathrm{C}$, particles were found to be around $12 \mathrm{~nm}$. This trend was observed for over 40 different materials; metals, semiconductors, dielectrics and ferrites.

Sol-gel synthesis falls under wet chemical techniques, and is widely employed in the field of nanoparticle preparation. Precursors develop from a simple solution, to a more complex network of discrete particles or chains, with the addition of a morphology controlling agent (sometimes termed 'surfactant') in a thermally controlled environment. A limitation of the solgel method is the activation (or burning off) of the surfactant - which can often increase particle size at high temperatures. This must be done since the agent is simply a cast for the particles to form in, and should not be included in experiments - especially in water splitting since some are organic and will act as electron donors. Parida et al. demonstrated that at an activation temperature of $500{ }^{\circ} \mathrm{C}, \mathrm{LaFeO}_{3}$ exhibited a $50 \%$ increase in $\mathrm{H}_{2} / \mathrm{O}_{2}$ yield when compared with samples which were treated at $900{ }^{\circ} \mathrm{C}$. particles of the lower temperature samples were also 4 times smaller and had a surface area which was over 5 times larger ${ }^{32}$.

It is important to take into account the amount of cocatalyst on the surface; reports in which cocatalysts are used often vary dramatically in terms of loading (in wt\% or mol\%), but commonly reach a peak, depending on the type of photocatalyst and particle size ${ }^{139}$. Too little cocatalyst results in incomplete coverage, whilst too much cocatalyst results in the surface of the photocatalyst being covered, and unable to absorb enough light to facilitate redox reactions.

Surface area can also play an important factor in determining the activity of a photocatalyst, with contradicting theories. This is evident in reports; Kudo et al. state that their large $\mathrm{BiVO}_{4}$ particles with a small surface area have higher $\mathrm{O}_{2}$ evolution rates than smaller particles with a larger surface area $^{31}$. Contradicting this, Ye et al. find that their $\mathrm{NaNbO}_{3}$ samples experience a 3 fold increase in $\mathrm{O}_{2}$ production and 6 fold hydrogen increase when the surface area of the samples are increased ${ }^{139}$. Another group 
reported a 72 fold increase in hydrogen production on nanocrystalline $\mathrm{La}_{2} \mathrm{Ti}_{2} \mathrm{O}_{7}$ when the surface area was increased from $1 \mathrm{~m}^{2} \mathrm{~g}^{-1}$ to $52 \mathrm{~m}^{2} \mathrm{~g}^{-1} \mathrm{using}$ a hydrothermal method as opposed to the conventional solid state reaction ${ }^{199}$. It is important to get a balance between particle size and photocatalytic activity, however, it is even more important in photocatalysis that there is a good degree of crystallinity, as this will decrease recombination between charge carriers. Conversely, a high surface area would be advantageous in degrading organic complexes since surface adsorption is the dominating factor.

In section 2.3.2, it was stated that different facets of $\mathrm{Ag}_{3} \mathrm{PO}_{4}$ showed varying activity for the degradation of organic contaminants, and this correlated with the surface energy of the facet. In fact, this is not the first example of facet enhanced photocatalysis. Normally, conventionally reactive facets are diminished during the crystal growth process due to surface energy minimisation. However, manipulating exposing facets on surfaces is a technique which has been investigated in sensing ${ }^{200}$, electronics ${ }^{201}$, magnetic memory devices ${ }^{202}$, and catalysis ${ }^{203}$ - with catalysis being the most relevant to semiconductor photocatalysis. Therefore under the correct conditions, the exposing facets can be controlled on metallic and metal oxide surfaces, often using a surfactant (morphology controlling agent). For example, anatase $\mathrm{TiO}_{2}$ crystals are often composed of thermodynamically stable $\{101\}$ facets, with an average surface energy of $0.44 \mathrm{~J} \mathrm{~m}^{-2}$. Very recently, led by theoretical predictions, $\{001\}$ facets on $\mathrm{TiO}_{2}$ were successfully prepared by using fluorine ions as a capping agent. The $\{001\}$ facets possess a much higher catalytic activity under UV light due to higher surface energy, calculated to be $0.90 \mathrm{~J} \mathrm{~m}^{-2}$ (over 2 times higher than $\{101\})^{204}$. This finding was supported by many other groups who demonstrated that high energy, low index facets of $\mathrm{TiO}_{2}$, are more reactive for both hydrogen evolution and organic degradation reactions ${ }^{205,206}$.

In order to attain specifically terminated facets, morphology controlling agents (MCAs), such as organic surfactants (e.g. PVP), or capping agents (e.g. fluorine ions) are commonly employed. Due to strong interaction with the substrate, complete removal of these MCAs is very challenging, but absolutely essential for effective catalytic activity in water splitting. One of the major research obstacles facing researchers today is developing a facile, MCA free synthesis route to attain clean, reactive facets, for example, by kinetic control ${ }^{207}$. In order to remove some surfactants, it is possible to simply prepare a suitable calcination process - however, there is a risk that 
the high energy facets will diminish due to surface reconstruction aided by an increased temperature.

Apart from $\mathrm{Ag}_{3} \mathrm{PO}_{4}$, faceted $\mathrm{BiVO}_{4}$ crystals have also been fabricated for the photooxidation of water under visible light. $\mathrm{Xi}$ et al reported the synthesis of monoclinic bismuth vanadate $\left(\mathrm{m}-\mathrm{BiVO}_{4}\right)$ nanoplates with exposed $\{001\}$ facets, in the absence of traditional surfactants ${ }^{208}$. Using ethanolamine and water as an azeotropic solvent, a bismuth vanadate precursor was made, and then crystallised in hydrothermal reactor at a relatively low temperature $\left(160{ }^{\circ} \mathrm{C} / 12\right.$ hours $)$. $\{001\}$ faceted $\mathrm{m}-\mathrm{BiVO}_{4}$ demonstrated superior photodegradation of $\mathrm{RhB}$, and also for the photooxidation of water. The authors state that the superior activity could stem from an increase in surface energy, or, an increase in hydrophobicity as a result of a low index exposing facet due to a different surface structure.

\subsubsection{Conclusions}

It is evident from the current review that in field of photocatalytic water splitting, not one system can demonstrate efficiency, cost-effectiveness and robustness on a scale which would prove economically viable $(10 \%$ minimum solar-to-hydrogen conversion efficiency). However, it is agreed in the field that a photocatalyst which responds to visible light is a must; ultraviolet radiation does not make up enough of the electromagnetic (EM) spectrum incident on the Earth's surface. By studying UV-active materials, of which some have excellent charge separation characteristics, and modifying them, the chance of attaining the set goal will dramatically increase.

Furthermore, it is evident that finding a 'perfect' photocatalyst which can split water into its constituent parts under visible light would be unfeasible at this present moment. One of the more promising areas of visible light water splitting is the development of photocatalysts which participate in a half reaction, and can be coupled to either a PEC/solar cell, or in a colloidal Z-Scheme. Therefore, based on the review of the current efficient photocatalysts of the present day, it appears the most suitable strategy would be to develop highly efficient, separate, photocatalysts for oxygen production and hydrogen production, exactly mimicking nature photosynthesis system, then in turn use high efficiency photocatalysts in order to create new Z-Scheme systems. This strategy has been applied in the thesis. 


\section{Experimental development}

\subsection{Reaction system}

In order to perform photocatalytic tests on compounds, a suitable reactor was fabricated based on previous successful designs in the literature $e^{7,15,209,210}$. The photocatalytic water splitting reaction is normally monitored by recording amount of product formed in a relatively small fixed volume batch reactor, over a set period of time. Therefore it is suitable to use a simple gas tight batch reactor for lab based testing, with an optical window for illumination. Most reactors used in the field can either be externally illuminated by a light source from the top down, or using a horizontal window (internal illumination is not considered). In this study, a side window will be used, as the choice of stirring method causes a vortex, which drives water and semiconductor particle away from the centre of the reactor to the perimeter. Thus, photons from the top down will be incident on the centre of the reactor, where the concentration of the solution is low (Figure 13).

(A)

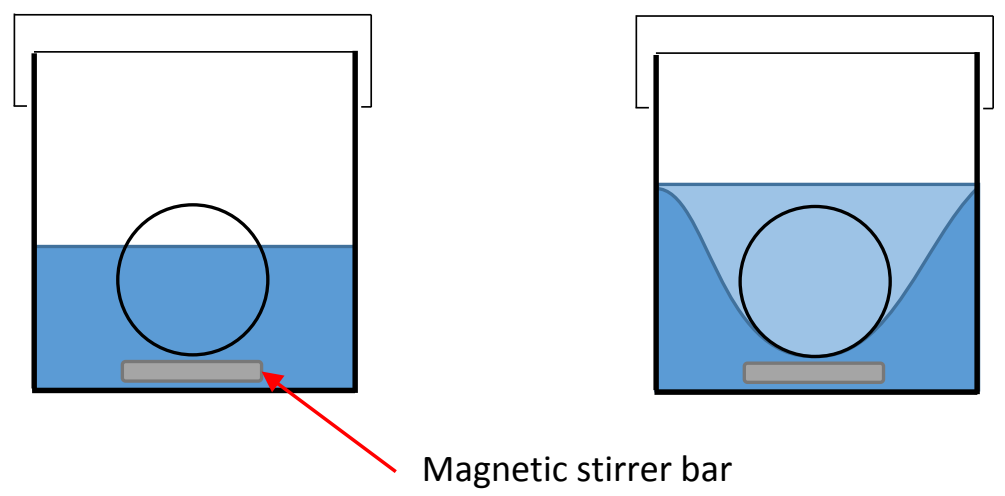

Figure 13. Schematic of proposed reactor (A) without magnetic stirring, (B) with stirring.

In order to stir the semiconductor-electrolyte (e.g. photocatalyst-water) mixture, a magnetic stirrer was used to drive an inert PTFE magnetic stirring bar in the solution, as shown in Figure 13. Conventional top down steel impeller blades would not be suitable for this application because of the risk of contamination of the blades with the semiconductor-electrolyte, 
i.e. through metallic leaching of nickel or chromium species. The nature of heterogeneous photocatalysis is such that the main interactions are that of the absorption of photons by the semiconductor, cleavage of water using charge carriers, and then release of product $\left(\mathrm{H}_{2}, \mathrm{O}_{2}\right.$ or both). In order for the photocatalyst to homogeneously absorb light (ignoring reflection), the photocatalyst particles must be mixed at a high enough radial velocity so that they do not sink to the bottom of the reactor, and maintain in constant motion close to the reactor walls. Having apparatus such as baffles would not benefit the absorption of light by the semiconductor since excessive mixing with water will not alter the reaction significantly. Water is later oxidised or reduced instantaneously, and gaseous product released as a dissolved gas in water. Therefore in this case, using a PTFE magnetic stirring bar to produce a radial flow, and thus induce solid body rotation in the liquid - which is enough to form a temporary suspension - is more than satisfactory. More complex mixing is not necessary.

\subsubsection{Reactor}

A borosilicate cylindrical glass reactor was designed and then handmade by Labglass Ltd $(4.5 \mathrm{~cm}$ radius, $11.5 \mathrm{~cm}$ height). The total volume was 730 $\mathbf{c m}^{3}$, including headspace (calculated by water displacement). The vessel is fitted with a flat high purity borosilicate side window, which is slightly bigger than the beam of the light source $(40 \mathrm{~mm} \Phi$ reactor window, $33 \mathrm{~mm}$ $\Phi$ beam). High purity borosilicate is suitable for a photocatalytic reactions as the low impurity content (such as iron) allows both UV and visible wavelengths of light to pass through without any absorption. 


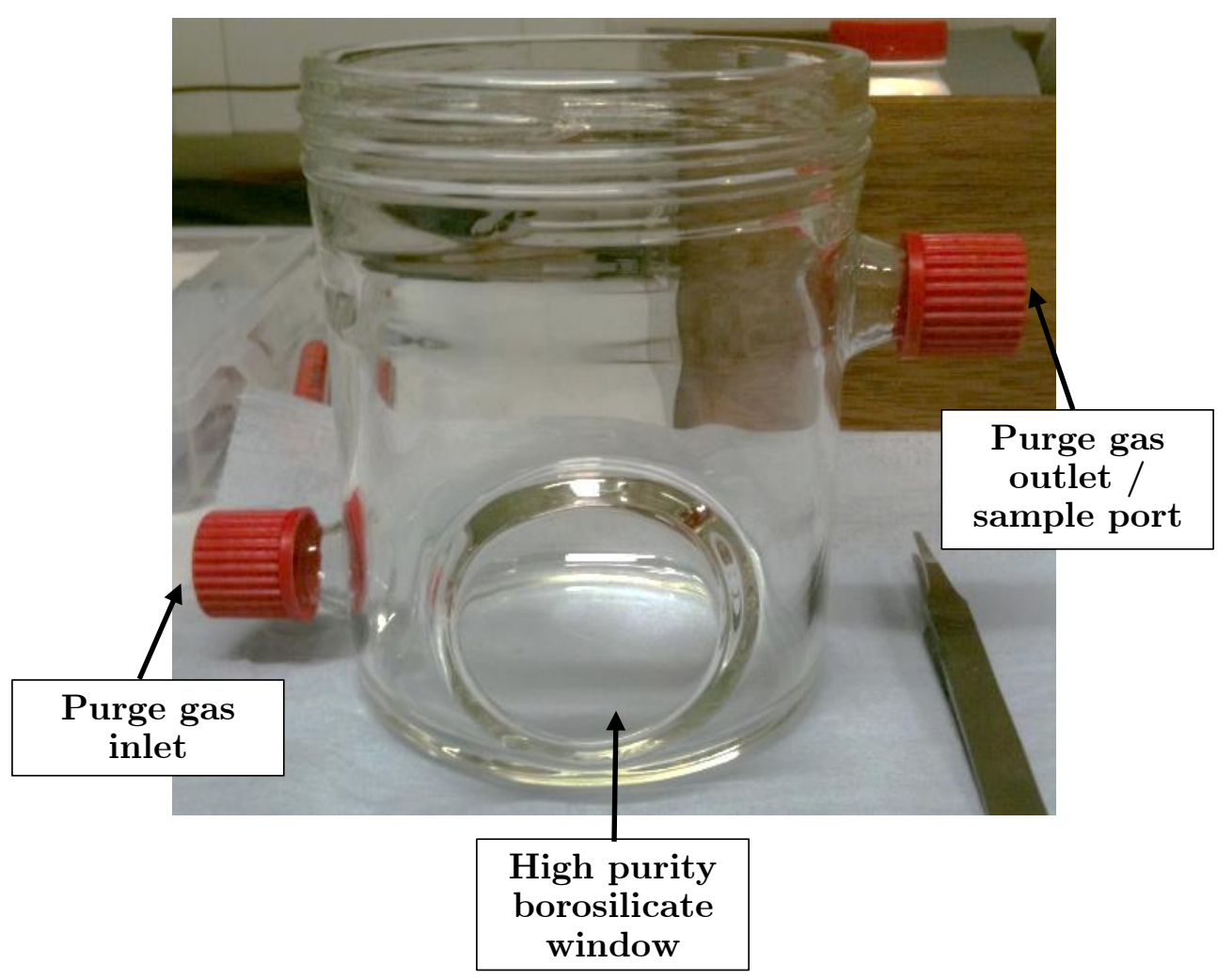

Figure 14. Borosilicate reactor for water splitting batch reactions.

The reactor was also fitted with two side ports (fitted with GL18 aperture caps and silicon septa) for purging and gas sampling. The top of the system was a PTFE screw thread aperture cap, with a detachable transparent borosilicate window. The reactor sealing mechanism was originally a $7 \mathrm{~mm}$ depth silicon ring which fitted between the glass and the top PTFE aperture cap. However, it was discovered that the sealing provided by the company was insufficient; the sealing would become dislodged during purging, and also during experiments. Despite numerous replacements from LabGlass, the sealing was a continuous problem and frequently disrupted and forced experiments to be stopped. Ultimately a solution was found whereby a very thin piece of silicon $(0.5 \mathrm{~mm}$, Altec Ltd) was cut to fit the reactor diameter. This method of sealing provided not only an airtight seal throughout the experiment, but also was able to withstand higher pressures generated when a high purging flow rate (in order to accelerate purge time). 


\subsubsection{Light Source}

Two different xenon (Xe) lamps were purchased from Newport Spectra and TrusTech to act as artificial light sources. Xe lamps have a spectral profile akin to that of the solar spectrum, with intensity less than $20 \mathrm{~mW} \mathrm{~m}^{-2} \mathbf{n m}$ ${ }^{1}$ at $250<\lambda<800 \mathrm{~nm}^{15}$. A $300 \mathrm{~W}$ Xe lamp (TrusTech PLS-SXE 300/300UV) was used for oxygen, hydrogen, and water splitting, and also for calculating solar-to-hydrogen conversion efficiency. The higher power lamp source will in theory enable the probing of photocatalysts whose efficiencies are considerably small, as a larger photon flux will increase the rate of water splitting. A $150 \mathrm{~W}$ Xe lamp (Newport 6256 150WXe) was utilised for IQY measurements; the lower intensity of the $150 \mathrm{~W}$ Xe lamp prevented damage to the band pass filters, which absorb considerable amounts of light, up to $90 \%$.

The power from the lamp was calculated using a Silicon photodiode detector $(190-1110 \mathrm{~nm})$, with built in attenuator, connected to a handheld digital power meter (both purchased from Newport Spectra). Various long pass filters were used, from 400 to $550 \mathrm{~nm}$, supplied by Comar Optics; enabling the selective use of either full arc or restricted visible light. Similarly, band pass filters were used in IQY measurements. These filters have a quoted centre wavelength, for example $400 \mathrm{~nm}$, and the width of all pass-bands are $10 \mathrm{~nm}(395-405 \mathrm{~nm})$. 


\subsection{Gas Chromatography: selection and calibration}

\subsubsection{Gas Chromatography setup}

A Varian 450 gas chromatograph (GC) was used to analyse the amounts of gaseous products from water splitting reactions (Figure 15), and to monitor nitrogen levels within a batch reactor. Samples were taken by using a gastight syringe (Hamilton ${ }^{\circledR} 1000 \mu \mathrm{L}$ ). The GC was fitted with a TCD and molecular sieve 5A column, running with argon carrier gas (zero grade). As discussed in section 2.2.2, argon is a suitable carrier gas for analysing concentrations of hydrogen, oxygen and nitrogen, due to the sufficiently low thermal conductivity - therefore all produced peaks will be positive, making integration easier during analysis (Table 2). Helium is an alternative, but is slightly more expensive, and would also yield negative peaks for oxygen and nitrogen due to the higher thermal conductivity, which would then be problematic during peak analysis. For these reasons it was not selected.

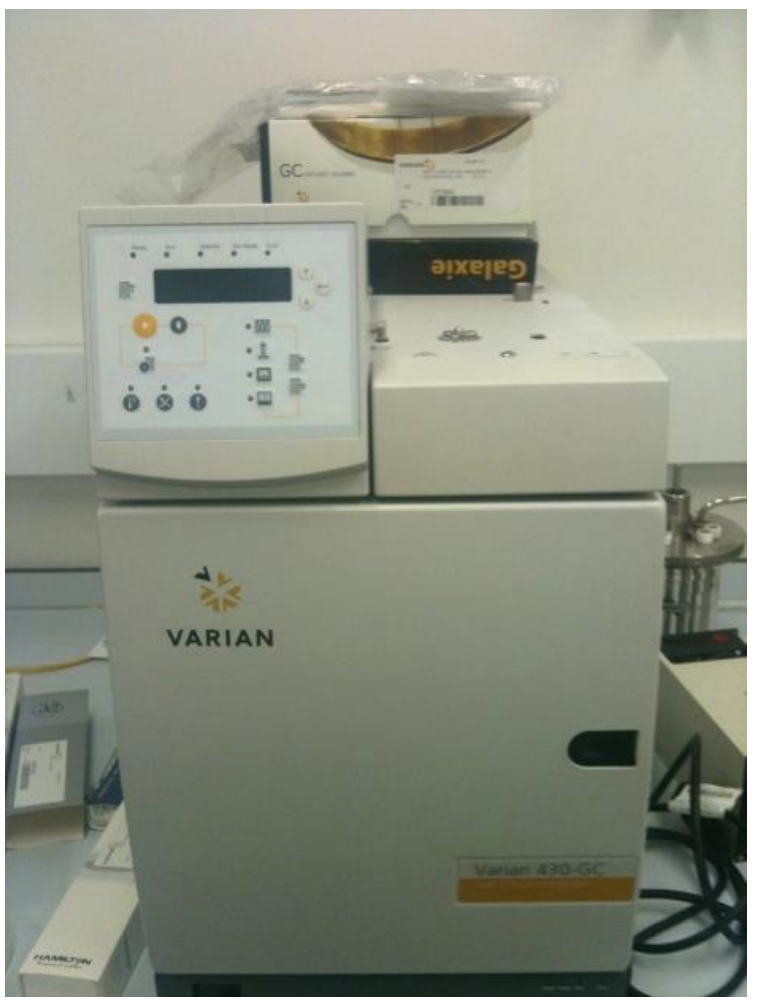

Figure 15. Photograph of the gas chromatograph unit used during photocatalysis experiments. 
Table 2. Gases and corresponding thermal conductivity at $\mathrm{STP}^{211}$.

\begin{tabular}{|c|c|}
\hline Gas & Thermal conductivity $\left(\mathrm{W} \mathbf{m}^{-1} \mathrm{~K}^{-1}\right)$ \\
\hline Hydrogen & 0.1805 \\
\hline Helium & 0.1513 \\
\hline Nitrogen & 0.0259 \\
\hline Oxygen & 0.0266 \\
\hline Argon & 0.0177 \\
\hline
\end{tabular}

The signal from the GC's TCD produces a chromatogram on the Varian 450-GC software (an idealised version is shown in Figure 16). The chromatograph is a plot of signal $(\mu \mathrm{V})$ versus time (minutes); whereby the area under the signal ( $\mu \mathrm{V} . \mathrm{min})$ is proportional to the concentration of a specific gas, and each separate signal is separated in time, denoting different gases. By selecting an appropriate baseline, and performing an automatic integration via the software, an accurate signal area can be acquired.

In order to establish a reasonable signal, parameters were tuned to optimise peak separation, signal-to-noise ratio, and baseline fluctuations. Many water splitting experiments documented in the literature record gas concentrations at either 15 or 30 minute intervals, with some elongated experiments recording every hour ${ }^{209}$. Therefore the total run time for each chromatogram should be less than $\mathbf{1 5}$ minutes, i.e. all necessary gases should appear in a 10 to 15 minute window, or have a retention time (RT) less than 15 minutes. In gas chromatography, the parameters which governs RT of a gas are oven temperature, pressure and flow rate of carrier gas inside the column - two of which were consequently set to $50{ }^{\circ} \mathrm{C}$, and 50 PSI to enable all gases (hydrogen, oxygen, nitrogen) to come through within a 10 minute window. Signal-to-noise ratio and baseline fluctuations can be controlled by adjusting flow rate and TCD filament temperature. After optimisation, the most efficient flow rate was $10 \mathrm{~cm}^{3} \mathrm{~min}^{-1}$ and $180{ }^{\circ} \mathrm{C}$. In general, increasing the flow rate through the TCD decreased peak width, but dramatically decreased peak height/response. Therefore a low flow rate was chosen so that small concentrations of gases could be detected. 


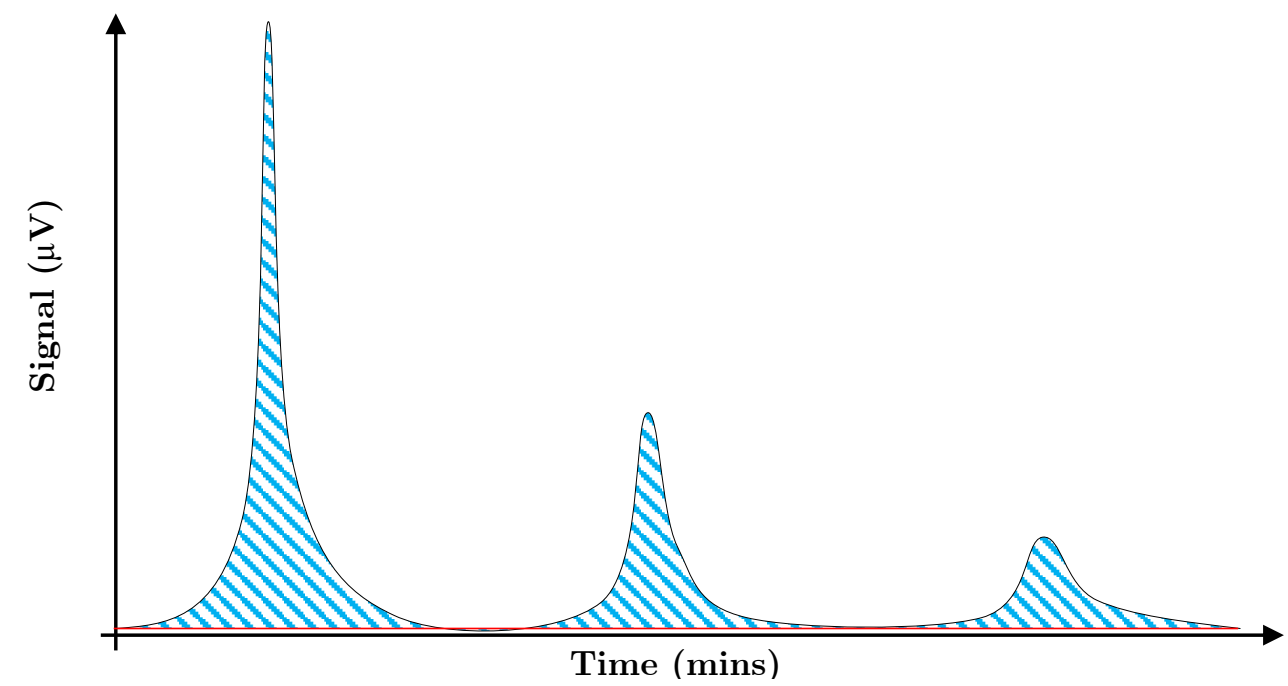

Figure 16. Idealised example chromatogram. A baseline is indicated by a horizontal red line, and integrated area under the curve (in $\mu \mathrm{V} . \mathrm{min}$ ) is indicated by a shaded blue area.

\subsubsection{Standard gas and calibration}

In order to correlate the response generated by the TCD on the GC to the actual gas composition, a calibration curve is used. By injecting known concentrations (and thus known molar amounts) of gas, and then monitoring the response, a graph of area $(y)$ versus concentration $(x)$ can be plotted. Then, by solving the equation of a line $(y=m x+c)$, for any area, a concentration can be calculated.

To then acquire an accurate known concentration, a suitable standard gas was purchased from BOC. It comprises of $99 \%$ zero grade argon, with appropriate amounts of $\mathrm{H}_{2}(4000 \mathrm{ppm})$ and $\mathrm{O}_{2}(2000 \mathrm{ppm})$, among other gases which might be produced as by-products in a half reaction in the presence of charge scavenger (1000 ppm CO, $1000 \mathrm{ppm} \mathrm{CO}_{2}$ and $2000 \mathrm{ppm}$ methane). By injecting different volumes of this gas into the GC, a calibration curve can be built (Figure 17), along with a response factor (R). The $\mathbf{R}$ factor is calculated by plotting amount of gas versus area, then solving the equation of the straight line for where the line intercepts the $\mathrm{x}$ axis. Generally the response factor (essentially an offset error) should be as 
close to zero as possible, implying a true linear relationship between gas amount and peak area. In reality however, this is not the case, due to errors in manual syringe sampling, TCD response and for oxygen error - a residual amount of air left in the syringe (dead volume). However, these can be corrected for simply using the R-factor in calculating the amount of gas being measured.

To establish a sampling error, 10 different samples of $0.5 \mathrm{~cm}^{3}$ standard gas was injected, and then average, standard deviation (SD, $\sigma)$ and percentage error was calculated (Table 3). The percentage error was calculated by dividing the SD by the mean.

Table 3. Area sampling data for $\mathrm{H}_{2}$ and $\mathrm{O}_{2}\left(0.5 \mathrm{~cm}^{3}\right)$. SD was taken for entire population

\begin{tabular}{|c|c|c|}
\hline & \multicolumn{2}{|c|}{ Area (uV.min) } \\
\hline & $\mathrm{H}_{2}$ & $\mathrm{O}_{2}$ \\
\hline & 4896.1 & 390.5 \\
\hline & 4834.4 & 394.5 \\
\hline & 4831.0 & 389.2 \\
& 4885.1 & 388.2 \\
\hline & 4876.7 & 399.5 \\
\hline & 4867.5 & 397.6 \\
\hline & 4845.9 & 391.1 \\
\hline & 4849.8 & 393.3 \\
\hline & 4888.6 & 393.9 \\
\hline & 4820.3 & 399.0 \\
\hline Mean & 4859.5 & 393.7 \\
\hline$\sigma$ & 25.4 & 3.8 \\
\hline \% & $0.52 \%$ & $0.97 \%$ \\
\hline Error & & \\
\hline
\end{tabular}

Using Table 3 , it is possible to apply a percentage error on both future $\mathbf{H}_{2}$ and $\mathrm{O}_{2}$ sampling data, which can be applied to calibration curves (Figure 17). 


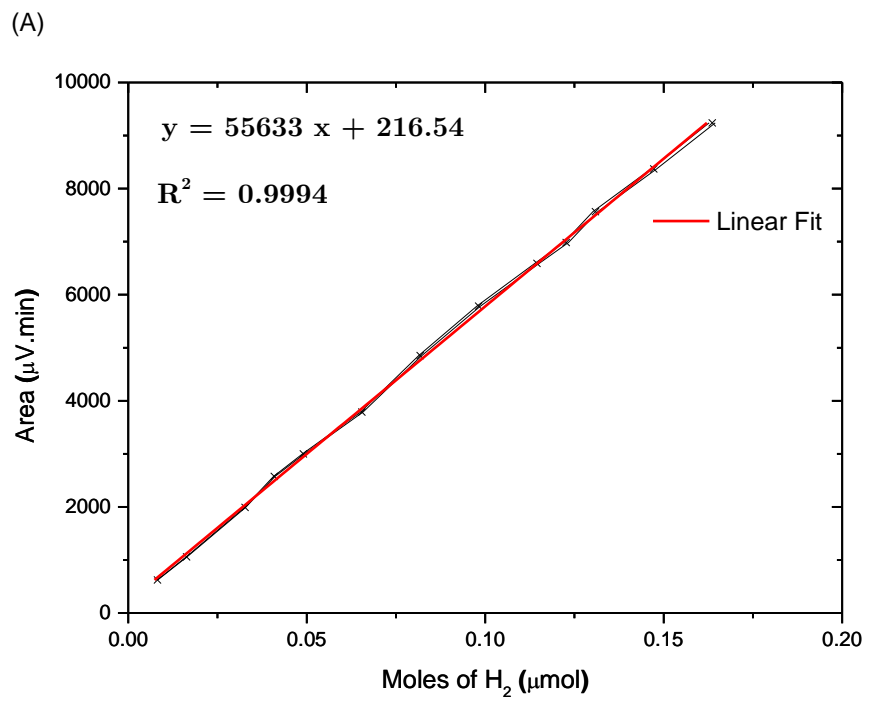

(B)

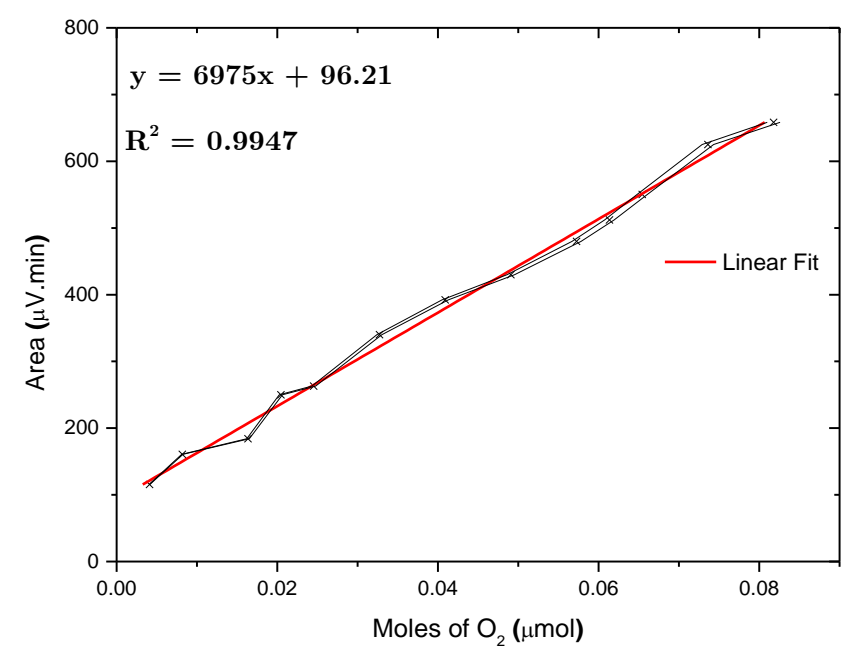

Figure 17. GC area vs. molar amount calibration curves for (A) hydrogen and (B) oxygen. Equations of linear fits and $R^{2}$ values are noted in the upper left hand corner of the figure. 
Table 4. Data table for Figure 17.

\begin{tabular}{|c|c|c|c|c|c|}
\hline \multirow{2}{*}{$\begin{array}{c}\text { Hydrogen } \\
\text { Syringe } \\
\text { volume }\left(\mathbf{c m}^{3}\right)\end{array}$} & \multirow[b]{2}{*}{$\begin{array}{c}\text { Amount } \\
\text { of } \mathbf{H}_{2} \\
\left(\mathbf{c m}^{3}\right)\end{array}$} & \multirow[b]{2}{*}{$\begin{array}{c}\text { Amount of } \\
\mathbf{H}_{2} \times \mathbf{1 0}^{-9}\left(\mathbf{m}^{3}\right)\end{array}$} & \multirow[b]{2}{*}{$\begin{array}{l}\text { Moles of } \\
\mathbf{H}_{2} \times 10^{-8}\end{array}$} & \multirow[b]{2}{*}{$\begin{array}{l}\text { Moles of } \\
\mathbf{H}_{2}(\mu \mathrm{M})\end{array}$} & \multirow[b]{2}{*}{$\begin{array}{c}\text { Area } \\
(\mu \text { V.min })\end{array}$} \\
\hline & & & & & \\
\hline 0.05 & 0.0002 & 0.2 & 0.82 & 0.008 & 623.4 \\
\hline 0.1 & 0.0004 & 0.4 & 1.64 & 0.016 & 1059.5 \\
\hline 0.2 & 0.0008 & 0.8 & 3.27 & 0.033 & 1992.3 \\
\hline 0.25 & 0.0010 & 1.0 & 4.09 & 0.041 & 2575.9 \\
\hline 0.3 & 0.0012 & 1.2 & 4.91 & 0.049 & 3000.5 \\
\hline 0.4 & 0.0016 & 1.6 & 6.54 & 0.065 & 3787.9 \\
\hline 0.5 & 0.0020 & 2.0 & 8.18 & 0.082 & 4853.8 \\
\hline 0.6 & 0.0024 & 2.4 & 9.82 & 0.098 & 5784.0 \\
\hline 0.7 & 0.0028 & 2.8 & 11.50 & 0.115 & 6590.5 \\
\hline 0.75 & 0.0030 & 3.0 & 12.30 & 0.123 & 6982.6 \\
\hline 0.8 & 0.0032 & 3.2 & 13.10 & 0.131 & 7565.7 \\
\hline 0.9 & 0.0036 & 3.6 & 14.70 & 0.147 & 8372.0 \\
\hline 1 & 0.004 & 4.0 & 16.30 & 0.164 & 9237.7 \\
\hline
\end{tabular}

\begin{tabular}{|c|c|c|c|c|c|}
\hline Oxygen & & & & & \\
\hline $\begin{array}{l}\text { Syringe volume } \\
\qquad\left(\mathrm{cm}^{3}\right)\end{array}$ & $\begin{array}{c}\text { Amount } \\
\text { of } \mathbf{O}_{2} \\
\left(\mathbf{c m}^{3}\right)\end{array}$ & $\underset{\mathbf{O}_{2} \times 10^{-9}\left(\mathbf{m}^{3}\right)}{\text { Amount of }}$ & $\begin{array}{c}\text { Moles of } \\
\mathrm{O}_{2} \times 10^{-8}\end{array}$ & $\begin{array}{l}\text { Moles of } \\
\mathrm{O}_{2}(\mu \mathrm{M})\end{array}$ & $\begin{array}{c}\text { Area } \\
(\mu \text { V.min })\end{array}$ \\
\hline 0.05 & 0.0001 & 0.1 & 0.41 & 0.0041 & 115.5 \\
\hline 0.1 & 0.0002 & 0.2 & 0.82 & 0.0082 & 160.7 \\
\hline 0.2 & 0.0004 & 0.4 & 1.64 & 0.0164 & 184.1 \\
\hline 0.25 & 0.0005 & 0.5 & 2.04 & 0.0204 & 250.0 \\
\hline 0.3 & 0.0006 & 0.6 & 2.45 & 0.0245 & 263.1 \\
\hline 0.4 & 0.0008 & 0.8 & 3.27 & 0.0327 & 340.0 \\
\hline 0.5 & 0.0010 & 1.0 & 4.09 & 0.0409 & 392.4 \\
\hline 0.6 & 0.0012 & 1.2 & 4.91 & 0.0491 & 430.0 \\
\hline 0.7 & 0.0014 & 1.4 & 5.73 & 0.0573 & 480.1 \\
\hline 0.75 & 0.0015 & 1.5 & 6.13 & 0.0613 & 512.0 \\
\hline 0.8 & 0.0016 & 1.6 & 6.54 & 0.0654 & 550.3 \\
\hline 0.9 & 0.0018 & 1.8 & 7.36 & 0.0736 & 625.1 \\
\hline 1 & 0.0020 & 2.0 & 8.18 & 0.0818 & 658.4 \\
\hline
\end{tabular}


According to the linear fit statistics, an unknown molar amount of hydrogen or oxygen can be calculated by knowing the area under the curve from a gas sample. For example an area of $86,000 \mu \mathrm{V}$.min is recorded by the GC for hydrogen. Using the equation of the line, $y=55663 \mathrm{x}+216.54$;

Equation 31.

$$
\left(\frac{86000-216.54}{55663}\right)=1.54 \text { umol of hydrogen }
$$

The linear regression best fit line shows that for hydrogen calibration, $\mathbf{r}^{2}=$ 0.9994 , and for oxygen $r^{2}=0.9947$. These high values of $r^{2}$ also confirm that by knowing $\mathrm{y}$, predicting $\mathrm{x}$ using $\mathrm{y}=\mathrm{mx}+\mathrm{c}$ has a high degree of confidence/accuracy ${ }^{212}$. Essentially r-squared is a fraction which is used to determine how $\mathrm{x}$ changes linearly with $\mathrm{y}$.

It is important to separate the oxygen measured from the standard gas, and that from air, which is left in the syringe tip (dead volume). From Table 3 it is evident that even a small amount of air in the syringe tip can influence the calibration, increasing error - which is nearly double that of the standard error on a hydrogen sample, since no hydrogen exists freely in air. The air contaminant also comes from a small amount which is inside the injection port in the GC. All of which is taken into account for the calibration. The successful calibration of the GC means that gas amounts as low as $10^{-9}$ moles can be detected accurately. 


\title{
3.3 General characterisation
}

The following section details standard characterisation methods that are used for all necessary results sections, to avoid repetition.

\subsubsection{UV-Vis Spectrophotometry}

\begin{abstract}
Absorption, reflection and transmission spectra were collected from a Shimadzu UV-2550 spectrophotometer fitted with an integrating sphere. The software provided (UV-Probe 2.33) enabled reflection to be directly converted to absorption by the Kubelka-Munk transformation. Typically, data would be collected from 250 to $800 \mathrm{~nm}$, with an optimum slit with of $2 \mathrm{~nm}$. This reduces noise whilst not compromising the accuracy of the data. The frequency of the data was $0.5 \mathrm{~nm}$, as this was more than adequate enough to determine precise spectra.
\end{abstract}

\subsubsection{PXRD}

PXRD was performed on either a Rigaku RINT $2100(40 \mathrm{kV}, 40 \mathrm{~mA}$, using a Cu source with $K_{\alpha_{1}}=1.540562$ and $K_{\alpha_{2}}=1.544398$ ) or a Bruker D4 (40 $\mathrm{kV}, 30 \mathrm{~mA}$, using a $\mathrm{Cu}$ source with $\mathrm{K}_{\alpha_{1}}=1.54056$ and $\left.\mathrm{K}_{\alpha_{2}}=1.54439\right)$. A maximum $0.05^{\circ}$ step size was used, at 5 seconds per step, covering a maximum range of $0-90^{\circ}(2 \theta)$. Phase match and baseline corrections were performed on either MDI Jade, or Bruker's EVA software using the ICSD/JCPDS database. A powdered sample was flattened into an amorphous glass (Rigaku) or silicon (Bruker) well holder.

\subsubsection{FE-SEM}

A JEOL JSM-7401F was used for measuring particle size, examining agglomeration of particles and also performing EDX measurements. The main advantage of using a Field Emission-SEM is that a greater resolution is possible (up to 6 times greater than conventional SEMs). Furthermore, electrostatic charging is greatly reduced on poorly conductive samples because lower acceleration voltages, without compromising image quality. Carbon tape was used as a conductive adhesive for the powdered samples. 


\subsubsection{TEM}

Conventional TEM measurements were taken using a JEOL2010F, at an accelerating voltage of $200 \mathrm{keV}$. Powdered samples were diluted in chloroform, sonicated to disperse particle, and then dropwise added onto a conductive copper grid. Tilt studies and EDX measurements were also conducted on a JEOL2010F.

\subsubsection{BET specific surface area}

Specific surface area was calculated via the BET method, using $\mathrm{N}_{2}$ absorption by a Micromeritics TriStar 3000. Powdered samples were placed in a borosilicate vial, with a weight to surface area ratio of $1 \mathrm{~g}$ to $30 \mathrm{~m}^{2}$ in order to acquire suitable data. Values of $\mathbf{R}^{2}$ (linear regression) were tuned to be as close to 1 as possible, with all values $\geq 0.9998$.

\subsubsection{ATR-FTIR spectroscopy}

ATR-FTIR spectroscopy was performed on a Perkin-Elmer 1605 FT-IR spectrometer in the wavenumber range from $400-4000 \mathrm{~cm}^{-1}$ with a resolution of $0.5 \mathrm{~cm}^{-1}$. Powdered samples were placed on the ATR crystal, and then compressed using a flat axial screw. Spectra were compared with literature examples, as there was available universal 'search and match' library for the instrument used.

\subsubsection{Raman spectroscopy}

Raman spectroscopic measurements were performed on a Renishaw InVia Raman Microscope, using an $\mathrm{Ar}^{+} 514.5 \mathrm{~nm}$ excitation laser, and a wavenumber range from $100-2000 \mathrm{~cm}^{-1}$. A 'notch filter' was used to cut out Raleigh components, and a silicon standard was used for calibration at $520 \mathrm{~cm}^{-1}$.

\subsubsection{TGA-DSC-MS}


Thermo Gravimetric Analysis-Differential Scanning Calorimetry-Mass Spectroscopy (TGA-DSC-MS) was performed on a Netzsch Jupiter TGADSC, connected to a Netzsch Aeolius MS, in an inert He atmosphere. The data was then processed using the Netzsch 'Proteus' thermal analysis software. Precursors (urea, thiourea) were placed in an alumina crucible, and calcined from $26.9{ }^{\circ} \mathrm{C}$ to $600{ }^{\circ} \mathrm{C}$, over a period of 115 minutes. From the raw data, TGA data yields a plot of mass loss (\%) versus temperature, DSC yields heat change (exothermic/endothermic reaction) versus temperature information, and MS monitors mass number (ion current, nA) relative concentration versus temperature.

3.3.9 Zeta Potential (ZP) measurements

ZP measurements were performed using a Zetasizer nano ZS equipped with a He-Ne laser $633 \mathrm{~nm}$, maximum $4 \mathrm{~mW}$ power, and analysis was undertaken using the 'Zetasizer software'. Powdered samples were diluted in a $0.05 \mathrm{M}$ aqueous $\mathrm{NaCl}$ solution, which acted as an electrolyte, increasing conductivity. A $5 \mathrm{~cm}^{3}$ sample was then sonicated for 30 minutes, and then placed into a ZP cuvette/cell with gold connectors. ZP was monitored as $\mathrm{pH}$ was changed from neutral (starting at ca. 7.7) to both alkaline and acidic conditions using $\mathrm{NaOH}$ and $\mathrm{HCl}$ respectively (manual titration). For each $\mathrm{pH}$ point, 5 measurements were taken, and the mean then calculated. The isoelectric point (IEP) is defined as the point of zero change at a set $\mathrm{pH}$, and thus was recorded at $\mathrm{ZP}=0$.

\subsubsection{XPS}

XPS measurements were performed on a Thermoscientific XPS K-alpha surface analysis machine using an Al source. Analysis was performed on the Thermo Advantage software. After samples were placed under UHV, a sweep scan was performed from $100-4000 \mathrm{eV}$. Each sample was scanned 6 times at different points on the surface to eliminate point error and create an average. Specific elemental peaks were then identified, and analysed further.

\subsubsection{Elemental Analysis}

Elemental Analysis (EA) was performed on a Micro Elemental Analyzer (CE-400 CHN Analyser, Exeter Analytical Instruments). Accurate ( \pm 0.1\%) weight percentages of carbon, nitrogen, hydrogen and trace elements were converted to atomic percentages before analysis. 


\section{Oxygen evolving photocatalyst development}

This chapter details the development and understanding of the extremely efficient photocatalyst, $\mathrm{Ag}_{3} \mathrm{PO}_{4}$. Due to the lack of literature which followed on from the initial investigation (by Yi et al) exploring the photooxidation of water, it was envisaged that the preparation method was difficult to replicate $^{7}$. This was indeed the case, as results show there was considerable differences in oxygen evolution from water between samples synthesised using different phosphate precursor sources $\left(\mathrm{PO}_{4}^{-}\right)$, and different solvents. A method was established to fabricate a silver phosphate sample which had a similar roughly spherical morphology, and exhibited a photooxidative ability analogous to that published, using ethanol, $\mathrm{AgNO}_{3}$, and $\mathrm{Na}_{2} \mathrm{HPO}_{4}$. Most notably, when the phosphate precursor was changed to $\mathrm{H}_{3} \mathrm{PO}_{4}$, and using a large volume of ethanol was used, it was possible to slow the growth of $\mathrm{Ag}_{3} \mathrm{PO}_{4}$ crystals, and retain low index facets, resulting in the production of novel $\{111\}$ terminated tetrahedral $\mathrm{Ag}_{3} \mathrm{PO}_{4}$ crystals. By changing the concentration of $\mathrm{H}_{3} \mathrm{PO}_{4}$, and thus changing the reaction kinetics, it is possible to monitor the growth mechanism of $\mathrm{Ag}_{3} \mathrm{PO}_{4}$ crystals from tetrapods to tetrahedrons.

$\mathrm{Ag}_{3} \mathrm{PO}_{4}$ tetrahedrons demonstrate considerably higher oxygen evolution activity in comparison to roughly spherical particles, and other low index facets. DFT calculations were used to model the surface energy, and the hole mass of $\mathrm{Ag}_{3} \mathrm{PO}_{4}$ in different directions. It is concluded that a combination of high surface energy and low hole mass on $\mathrm{Ag}_{3} \mathrm{PO}_{4}\{111\}$ surfaces result in extremely high oxygen evolution from water, with an internal quantum yield of $98 \%{ }^{213}$. 


\subsection{Introduction}

Water photolysis for $\mathrm{H}_{2}$ fuel synthesis has the potential to solve both increasing demand of renewable energy and climate change caused by $\mathrm{CO}_{2}$ emissions. However the search for a solitary low cost semiconductor which has a band gap suitable for an efficient, neutral water splitting reaction under ambient conditions has been met with little success. Nature, however, demonstrates an efficient strategy to utilise solar irradiation (nearly unity IQY) by spatially and temporally separating electrons and holes in wireless photosynthesis reactions ${ }^{214}$. So, water splitting can be envisaged as two half reactions; water oxidation, and an equivalent of proton reduction to hydrogen fuel. The former of the two is much more challenging because one molecule of gaseous oxygen requires 4 holes, and occurs on a timescale ca. 5 orders of magnitude slower than $\mathrm{H}_{2}$ evolution, proven in both natural and artificial photosynthesis ${ }^{30,42,215}$. Therefore, finding an relatively cheap, robust and efficient water oxidation photocatalyst is widely accepted to be key to solar driven fuel synthesis, if it is to be commercial viable.

$\mathrm{Ag}_{3} \mathrm{PO}_{4}$ is used commonly in the pharmaceutical field as an antibacterial agent ${ }^{216}$, and is also considered to be an ideal candidate for water photooxidation due to an appropriate band gap position, non-toxicity, and high photocatalytic ability ${ }^{7,217}$. It is widely known that activity of a catalyst can be determined by its exposing facets. In order to attain specifically terminated facets, morphology controlling agents (MCAs), such as organic surfactants (e.g. PVP), or capping agents (e.g. fluorine ions ${ }^{204}$ ) are commonly employed. Due to strong interaction with the substrate, complete removal of these MCAs is very challenging, but absolutely essential for effective catalytic activity in water splitting. One of the major research obstacles facing researchers today is developing a facile, MCA-free synthesis route to attain clean, reactive facets, for example, by kinetic control $^{207}$.

We performed DFT calculations, and it was found that $\mathrm{Ag}_{3} \mathrm{PO}_{4}$ possessed an anisotropic hole mass $\left(\mathrm{m}_{\mathrm{h}}{ }^{+}\right)$, and variable surface energy depending on the exposing facets - in particular that the $\{111\}$ crystal planes had a large surface energy. Previously, photocatalytic organic decomposition on faceted $\mathrm{Ag}_{3} \mathrm{PO}_{4}$ has been reported. For example, Wang and co-workers synthesised tetrahedral shaped particles after attempting to rejuvenate $\mathbf{A g}-\mathrm{Ag}_{3} \mathrm{PO}_{4}$. Due to the presence of silver metal within the $\mathrm{Ag}_{3} \mathrm{PO}_{4}$ particles, they exhibited lower activity for the degradation of the dye $\mathrm{RhB}$ in comparison to mixed 
faceted particles ${ }^{218}$. Bi and co-workers have also compared photocatalytic degradation reactions over cubic and rhombic dodecahedral silver phosphate structures ${ }^{149}$. However, to date, there have not been reports on the influence of different exposing $\mathrm{Ag}_{3} \mathrm{PO}_{4}$ facets on water photooxidation, which is a well-known rate determining step in photocatalytic water splitting and has different requirements in comparison to organic decomposition reactions. Therefore, attempts were made to manipulate the material facets in order to control both charge transport (in particular hole transport and mobility) and surface photooxidation reactions using a facile and environmentally friendly approach. Herein, this chapter shows the manipulation of $\mathrm{Ag}_{3} \mathrm{PO}_{4}$ facets, and for the first time investigates their individual ability for water photooxidation and details the underlying mechanisms which control oxygen production; specifically between different low index facets of $\mathrm{Ag}_{3} \mathrm{PO}_{4}$ using diverse characterisation methods.

\subsection{Methodology}

\subsubsection{Photocatalytic analysis}

Photooxidation reactions were carried out in both the UK and Japan to verify the continuity of results, which were deemed to be consistent. For a typical oxygen evolution experiment, $0.2 \mathrm{~g}$ of $\mathrm{Ag}_{3} \mathrm{PO}_{4}$ powder was dispersed in $230 \mathrm{~cm}^{3}$ of deionised water, with an additional amount of $0.85 \mathrm{~g} \mathrm{AgNO}_{3}$ acting as an electron acceptor. $\mathrm{AgNO}_{3}$ was used as an electron scavenger in all standard oxygen evolution experiments as it was shown by Mills et al to be the most efficient, and convenient electron scavenger ${ }^{35}$. The whole reaction was carried out in a custom glass reactor. Before the solution was irradiated, the reactor was either connected to a vacuum system (Japan), and evacuated, or thoroughly purged with argon (UK) to remove all oxygen in the headspace of the reactor and dissolved oxygen in water. A baseline was taken to ensure that there was little or no detectable oxygen in the system. A 300W Xe lamp (UK: TrusTech PLS-SXE 300/300UV; Japan: Hayashi Tokei, Luminar Ace 210,) was used to irradiate the sample after a suitable baseline was obtained (power density full arc: ca. $500 \pm 25 \mathrm{~mW} \mathrm{~cm}$ ${ }^{2}, 420 \mathrm{~nm}$ filter: ca. $360 \pm 20 \mathrm{~mW} \mathrm{~cm}^{-2}$ ). A small sample of gas was sent to a gas chromatograph (UK: Varian 430-GC, TCD, Argon carrier gas, Japan: Shimadzu GC-8A, TCD, Argon carrier gas,) at specific intervals, upon which the concentration of oxygen was identified.

Internal quantum yield measurements were measured in the UK by inserting a band pass filter $(365,400,420,500,600 \mathrm{~nm})$ in front of a $150 \mathrm{~W}$ Xe light source (Newport Spectra) to obtain the correct wavelength. The 
light intensity was measured at 5 different points and then and average of the light intensity was then taken. The efficiency was then calculated by the standard formula quoted in literature, ignoring reflected photons ${ }^{15,38,219}$; $\operatorname{IQY}(\%)=4 \times$ number of $\mathrm{O}_{2}$ molecules detected divided by the number of photons absorbed by the photocatalyst. The reported turnover frequency (TOF $\min ^{-1}$ ) was calculated using the following formula as quoted in the literature ${ }^{15,38}$ : TOF $\left(\mathrm{min}^{-1}\right)=$ the ratio of number of reacted holes to number of atoms in photocatalyst, per minute over a three hour experiment. Turnover number was calculated using the same formula, negating time.

\subsubsection{Synthesis techniques}

$\mathrm{Ag}_{3} \mathrm{PO}_{4}$ was originally fabricated as an active photocatalyst by $\mathrm{Yi}$ et al using an ion-exchange method $^{7}$. The authors claim that by mixing/grinding stoichiometric amounts of either $\mathrm{Na}_{2} \mathrm{HPO}_{4}$ (anhydrous, 'dibasic') or $\mathrm{Na}_{3} \mathrm{PO}_{4} \cdot 12 \mathrm{H}_{2} \mathrm{O}$ (dodecahydrate, 'tribasic') with $\mathrm{AgNO}_{3}$, at room temperature using no solvents, a $\mathrm{Ag}_{3} \mathrm{PO}_{4}$ precipitate is formed. This precipitate is then washed with deionised water to remove unreacted precursors and dried at $70{ }^{\circ} \mathrm{C}$ to remove all water. This method (denoted 'A') was repeated with both precursors $(99.9 \%$ grade, purchased from Sigma-Aldrich and VWR), using a pestle and mortar for mixing the two solids. $\mathrm{NaH}_{2} \mathrm{PO}_{4}$ (anhydrous, 'monobasic', 99.9\%, VWR) was also mixed with $\mathrm{AgNO}_{3}$ in a similar manner for completion. Method A and subsequent methods B-D are summarised in Table 5.

This method was repeated with four phosphate precursors $\left(\mathrm{Na}_{3} \mathrm{PO}_{4}\right.$, $\mathrm{Na}_{2} \mathrm{HPO}_{4}, \mathrm{NaH}_{2} \mathrm{PO}_{4}, \mathrm{H}_{3} \mathrm{PO}_{4}$ ), but with the addition of $5 \mathrm{~cm}^{3}$ of ethanol or methanol (95\%, Merck Schuchardt) to aid ion-exchange during the mortar and pestle grinding process (denoted method ' $\mathrm{B}$ '). No powder sample was synthesised when $\mathrm{H}_{3} \mathrm{PO}_{4}$ was used, as the acidity of phosphoric acid (pH 1) prevented the formation of $\mathrm{Ag}_{3} \mathrm{PO}_{4}$.

In a different method (denoted 'C'), stoichiometric amounts of $\mathrm{Na}_{2} \mathrm{HPO}_{4}$ and $\mathrm{AgNO}_{3}$ were separately dissolved in $80 \mathrm{~cm}^{3}$ of ethanol in a conical flask, with the aid of a magnetic stirrer and magnetic stirring plate (approx. 1500 rpm). Then the $\mathrm{AgNO}_{3}$-ethanol solution was added dropwise to the phosphate-ethanol solution until a yellow $\mathrm{Ag}_{3} \mathrm{PO}_{4}$ precipitate was formed. The solution was both centrifuged and washed ( 3 times) immediately after precipitation, or after 1 hour of stirring. 
Table 5. Summary of results on initial $\mathrm{Ag}_{3} \mathrm{PO}_{4}$ studies using various phosphate precursors. $\mathrm{All} \mathrm{Ag}_{3} \mathrm{PO}_{4}$ compounds were synthesised using $\mathrm{AgNO}_{3}$ as the silver ion source.

\begin{tabular}{|c|c|c|c|c|c|}
\hline Method & $\begin{array}{c}\text { Sample } \\
\text { name }\end{array}$ & $\begin{array}{l}\text { Phosphate } \\
\text { precursor }\end{array}$ & $\begin{array}{c}\text { Synthesis } \\
\text { Method }\end{array}$ & $\begin{array}{c}\text { Initial oxygen evolution rate }\left(\mu \mathrm{mol} \mathrm{h} \mathrm{h}^{-1} \mathrm{~g}^{-1}\right) \\
\text { under visible light }(\lambda \geq 420 \mathrm{~nm}) . \text { Error }= \\
0.97 \%\end{array}$ & $\operatorname{BET~SSA~}\left(\mathbf{m}^{2} \mathbf{g}^{-1}\right)$ \\
\hline $\mathbf{A}$ & A1 & $\mathrm{Na}_{2} \mathrm{HPO}_{4}$ & \multirow{3}{*}{$\begin{array}{l}\text { Ground, } \\
\text { No } \\
\text { solvent }\end{array}$} & 733 & 1.12 \\
\hline $\mathbf{A}$ & A2 & $\mathrm{Na}_{3} \mathrm{PO}_{4} \cdot 12 \mathrm{H}_{2} \mathrm{O}$ & & 478 & 1.35 \\
\hline $\mathbf{A}$ & A3 & $\mathrm{NaH}_{2} \mathrm{PO}_{4}$ & & 687 & 0.98 \\
\hline B & B1 & $\mathrm{Na}_{2} \mathrm{HPO}_{4}$ & \multirow{2}{*}{$\begin{array}{l}\text { Ground, } \\
5 \mathrm{~cm}^{3} \\
\text { EtOH }\end{array}$} & 1357 & 1.01 \\
\hline B & B2 & $\mathrm{NaH}_{2} \mathrm{PO}_{4}$ & & 671 & 1.40 \\
\hline B & B3 & $\mathrm{Na}_{2} \mathrm{HPO}_{4}$ & $\begin{array}{c}\text { Ground, } \\
\mathrm{MeOH}\end{array}$ & 1159 & 1.29 \\
\hline $\mathbf{C}$ & C1 & $\mathrm{Na}_{2} \mathrm{HPO}_{4}$ & $\begin{array}{c}\text { Dissolved } \\
\text { in EtOH }\end{array}$ & 1418 & 0.91 \\
\hline $\mathbf{D}$ & Tetrahedron & $20 \mathrm{~cm}^{3} \mathrm{H}_{3} \mathrm{PO}_{4}$ & $\begin{array}{c}\text { Dissolved } \\
\text { in EtOH }\end{array}$ & 6074 & 1.01 \\
\hline
\end{tabular}


Using method ' $\mathrm{C}$ ' as a template, $\mathrm{Na}_{2} \mathrm{HPO}_{4}$ was replaced with another phosphate precursor which was free of sodium, $\mathrm{H}_{3} \mathrm{PO}_{4}(85 \%$, Acros Organics). This method (' $D$ ') involved the same initial steps in terms of dissolving both liquid $\mathrm{H}_{3} \mathrm{PO}_{4}$ (various volumes from $2 \mathrm{~cm}^{3}$ to $20 \mathrm{~cm}^{3}$ ) and $2 \mathrm{~g} \mathrm{AgNO}_{3}$ in separate ethanol solutions of $80 \mathrm{~cm}^{3}$ volume under vigorous stirring. Upon adding the $\mathrm{AgNO}_{3}$-ethanol solution to the $\mathrm{H}_{3} \mathrm{PO}_{4}$-ethanol solution, a yellow $\mathrm{Ag}_{3} \mathrm{PO}_{4}$ precipitate appeared, but then rapidly dissolved, most likely due to the $\mathrm{pH}$ of the $\mathrm{H}_{3} \mathrm{PO}_{4}$-ethanol solution (approx. $\mathrm{pH} 1$ ), as seen in method 'B'. After adding approximately $10 \mathrm{~cm}^{3}$ of $\mathrm{AgNO}_{3}$-ethanol solution, the $\mathrm{H}_{3} \mathrm{PO}_{4}$-ethanol solution turned slightly milk-white. If all 80 $\mathrm{cm}^{3}$ of the $\mathrm{AgNO}_{3}$-ethanol solution was added, the colour remained milk white; no precipitate was formed. If however, $10 \mathrm{~cm}^{3}$ of $\mathrm{AgNO}_{3}$-ethanol was added to the $\mathrm{H}_{3} \mathrm{PO}_{4}$-ethanol solution, and then a few drops of the solution added back into the bare $\mathrm{AgNO}_{3}$-ethanol solution, an instantaneous yellow precipitate was formed. After 1 hour of stirring the yellow precipitate turned dark green. The precipitates were centrifuged and washed with deionised water 3 times, before drying overnight in an oven at $70{ }^{\circ} \mathrm{C}$.

Rhombic dodecahedrons and cubic $\mathrm{Ag}_{3} \mathrm{PO}_{4}$ crystals were synthesised using a recipe based on a study by $\mathrm{Bi}$ et $\mathrm{al}^{149}$. The initial recipe described by the authors did not yield the required morphology, and therefore a different method was developed.

To synthesise $\mathrm{Ag}_{3} \mathrm{PO}_{4}$ cubes, $0.45 \mathrm{~g} \mathrm{AgNO}$ was dissolved in $50 \mathrm{~cm}^{3} \mathrm{DI}$ water. Meanwhile in a separate vial, $0.069 \mathrm{~cm}^{3} \mathrm{NH}_{3} \cdot \mathrm{H}_{2} \mathrm{O}$ was added dropwise to $10 \mathrm{~cm}^{3}$ DI water. The ammonia solution was then added to the silver nitrate solution, to which the resulting solution turned first brown $\left(\mathrm{Ag}_{2} \mathrm{O}\right)$, and then transparent $\left[\mathrm{Ag}\left(\mathrm{NH}_{3}\right)_{2}\right]^{+}$. This colour change can be explained by the $\mathrm{pH}$ mediated complexing of $\mathrm{Ag}^{+}$ions:

Initially, $\mathrm{NH}_{4} \mathrm{OH}$ dissociates and releases $\mathrm{OH}^{-}$ions

Equation 32.

$$
\mathrm{NH}_{3} \cdot \mathrm{H}_{2} \mathrm{O} \rightarrow \mathrm{NH}_{4} \mathrm{OH} \rightarrow \mathrm{NH}_{4}^{+}+\mathrm{OH}^{-}
$$

The $\mathrm{OH}^{-}$ions then react with silver ions (dissolved in the solution from $\left.\mathrm{AgNO}_{3}\right)$

Equation 33.

$$
2 \mathrm{Ag}^{+}+2 \mathrm{OH}^{-} \rightarrow \mathrm{Ag}_{2} \mathrm{O}+\mathrm{H}_{2} \mathrm{O}
$$


And finally as the $\mathrm{pH}$ becomes more basic with the addition of more $\mathrm{NH}_{4} \mathrm{OH}$

Equation 34.

$$
\mathrm{Ag}_{2} \mathrm{O}+4 \mathrm{NH}_{3}+\mathrm{H}_{2} \mathrm{O} \rightarrow 2\left[\mathrm{Ag}\left(\mathrm{NH}_{3}\right)_{2}\right]^{+}+2 \mathrm{OH}^{-}
$$

To this transparent solution, $0.15 \mathrm{M}$ of aqueous $\mathrm{Na}_{2} \mathrm{HPO}_{4}$ solution was added until the solution appeared yellow-green. The solution was stirred for 2 hours at $1000 \mathrm{rpm}$ using a magnetic stirrer-bar/plate, and subsequently washed and centrifuged 3 times to remove any unreacted product.

Bi et al used PVP to synthesise rhombic dodecahedrons, however, when this method was repeated, the desired morphology was not seen. To synthesise silver phosphate rhombic dodecahedrons, in the absence of the surfactant PVP, the following novel recipe was implemented. $0.6 \mathrm{~g} \mathrm{AgNO}_{3}$ was dissolved in $300 \mathrm{~cm}^{3}$ DI water. A $0.1 \mathrm{M}$ ammonium hydroxide solution was added until the silver nitrate solution turned brown, and then clear. This signifies the formation of a silver hydroxide species, and then a silver ammonia complex respectively. A $0.15 \mathrm{M} \mathrm{Na}_{2} \mathrm{HPO}_{4}\left(450 \mathrm{~cm}^{3}\right)$ was then added to the silver ammonia complex solution. In order to neutralise the $\mathrm{pH}, \mathrm{H}_{3} \mathrm{PO}_{4}$ was added dropwise, to which a bright yellow compound precipitated. The solution was also stirred for 2 hours at 1000 rpm using a magnetic stirrer-bar/plate, and subsequently washed and centrifuged 3 times to remove any unreacted product. 


\subsection{Results and discussion}

\subsubsection{Initial $\mathrm{Ag}_{3} \mathrm{PO}_{4}$ studies}

In order to establish a definitive baseline, three different synthesis methods (A - C) were attempted in order to acquire a sample with similar activity $\left(1272 \mu \mathrm{mol} \mathrm{h} \mathrm{h}^{-1} \mathrm{~g}^{-1}\right)$ reported by $\mathrm{Yi}$ et al under visible light $(\lambda>420 \mathrm{~nm})$. The results are summarised in Table 5 .

\subsubsection{Method 'A'}

The three samples fabricated using a solid mixing/grinding method (' $A$ ') in a mortar and pestle all yielded relatively large agglomerates of smaller, roughly spherical particles, as can be seen in Figure 18. All samples were confirmed my XRD to be phase pure body-centred cubic crystalline $\mathrm{Ag}_{3} \mathrm{PO}_{4}$ (6.004 ^̊, BCC, P-43n, JCPDS no.06-0505) (Figure 19 (A)), and possess an absorption profile characteristic of a semiconductor with a band gap to absorb light in the visible range (Figure 19 (B)). Particle size analysis was not performed as the difference between an agglomerate and a distinct particle could not be verified.
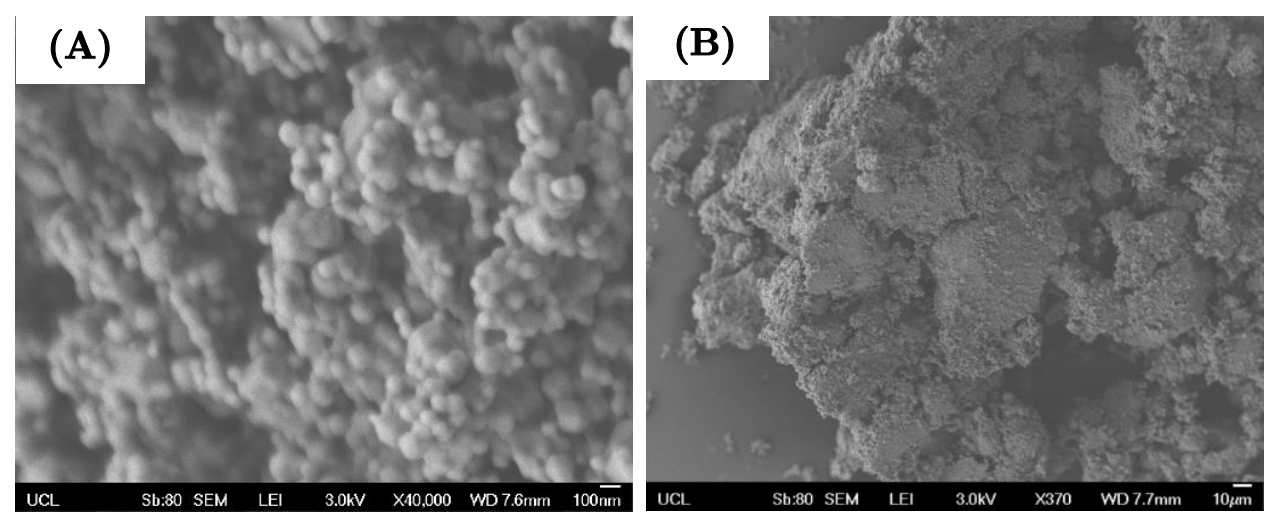

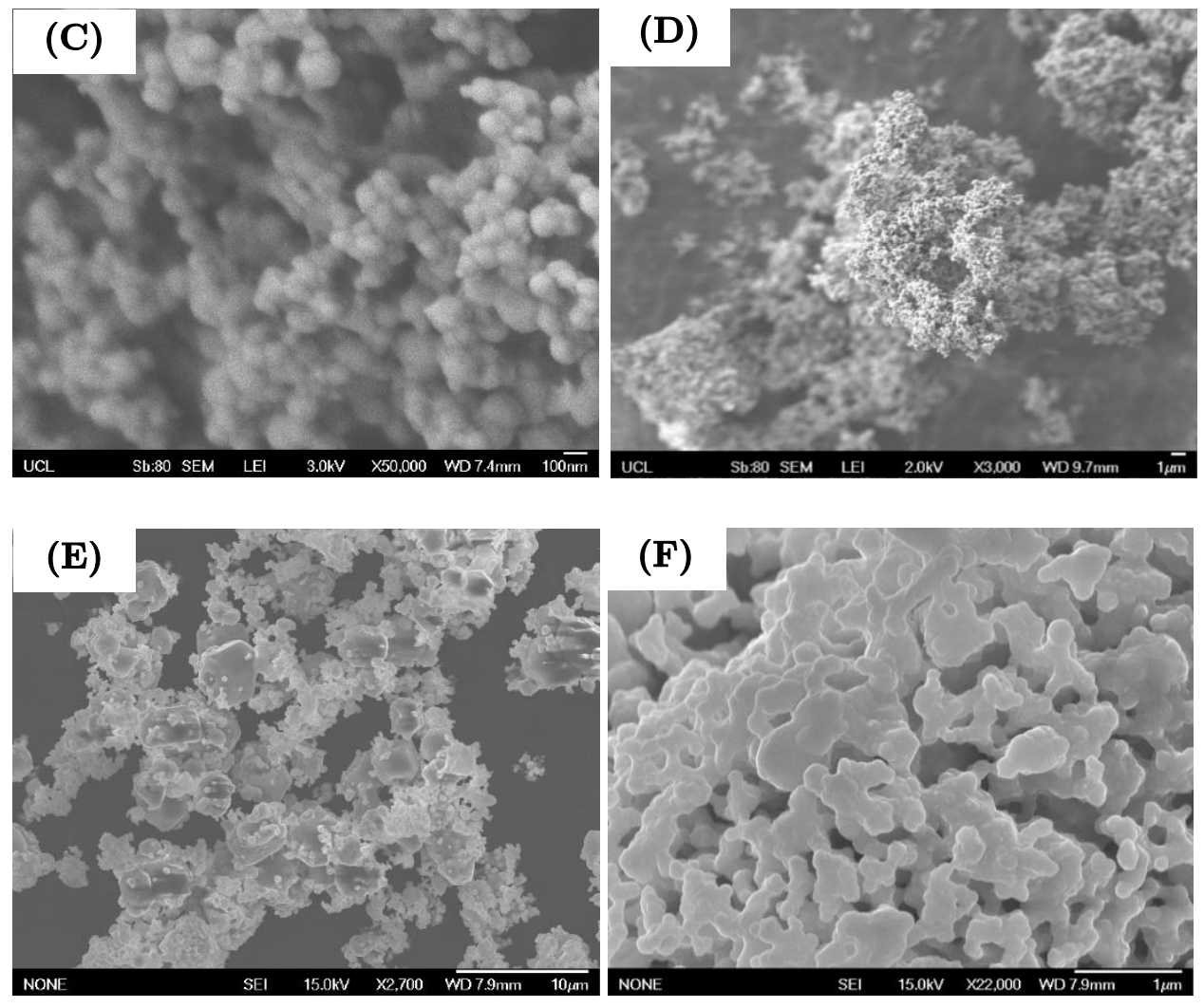

Figure 18. SEM micrographs of samples A1 (A \& B), A2 (C \& D), and A3 $(\mathrm{E} \& \mathrm{~F})$. 

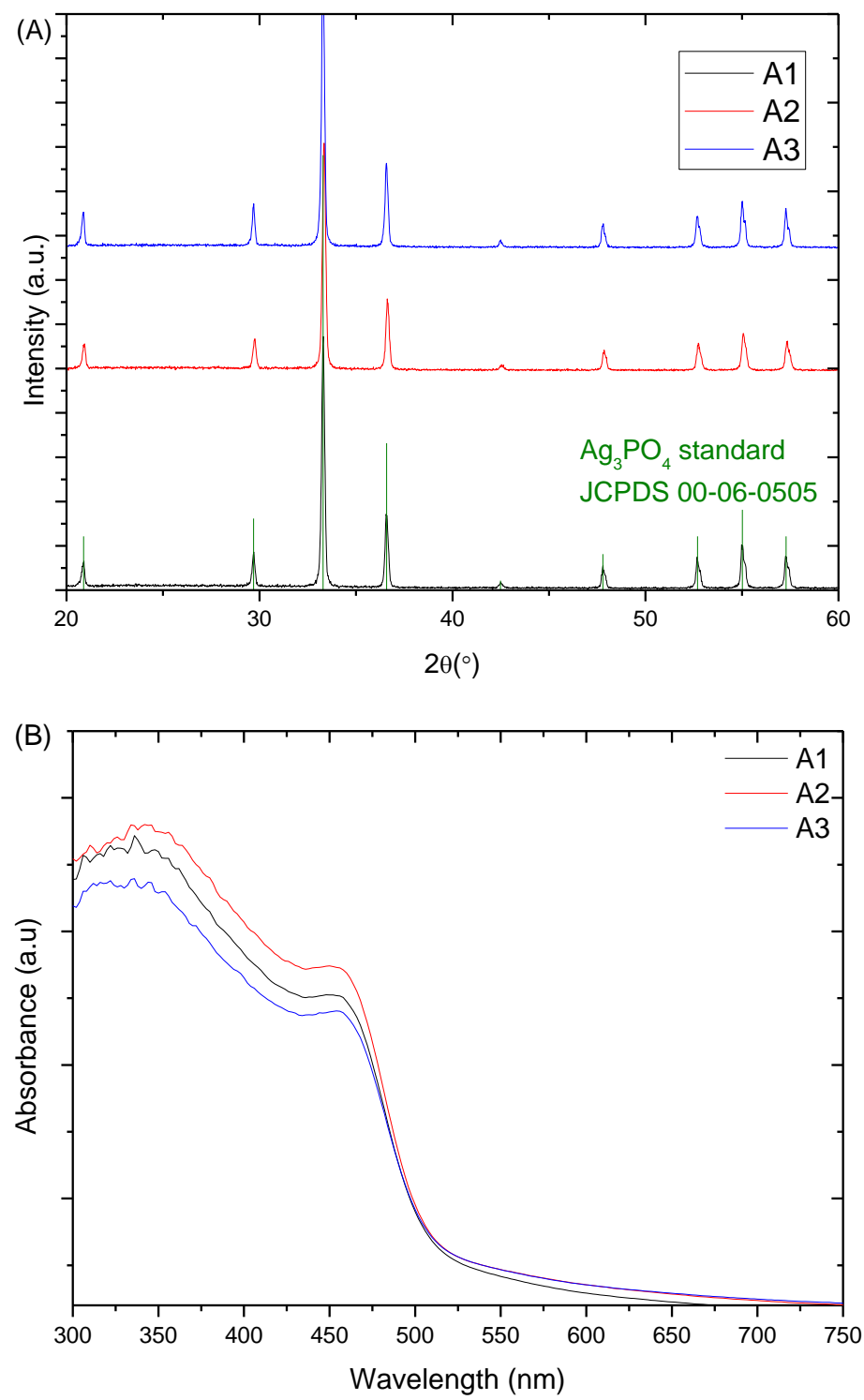

Figure 19. (A) XRD patterns of samples A1, A2 and A3. (B) UV-Vis absorbance spectra of samples A1, A2, and A3.

Since there was no correlation between oxygen evolution rate (OER) and BET SSA, it was theorised that either unreacted sodium or nitrate ions in the solution and at the surface could be hindering activity, by adsorbing on the surface of the photocatalyst and blocking active sites. Therefore, during the solid mixing process, a small amount of ethanol/methanol was added to facilitate better ion exchange and potentially remove $\mathrm{Na}$ ions from the surface. 


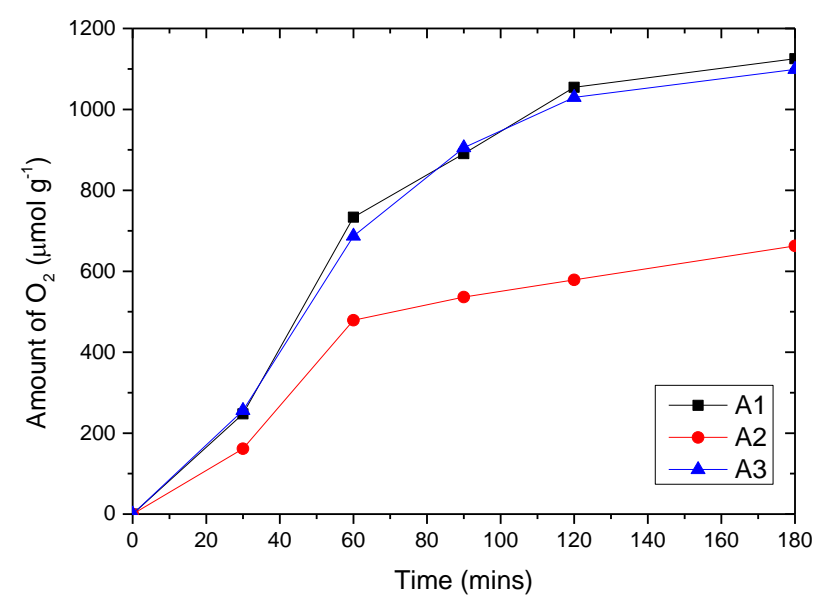

Figure 20. Oxygen evolution under visible light $(\lambda>420 \mathrm{~nm})$ from water, in the presence of $\mathrm{AgNO}_{3}(0.85 \mathrm{~g})$, at neutral $\mathrm{pH}$, using $0.2 \mathrm{~g}$ photocatalyst (A1, A2, A3).

\subsubsection{Method ' $B$ '}

Since sample A2, synthesised using $\mathrm{Na}_{3} \mathrm{PO}_{4} \cdot 12 \mathrm{H}_{2} \mathrm{O}$, displayed a poor OER, even with the largest surface area from the batch, it was decided to not use $\mathrm{Na}_{3} \mathrm{PO}_{4} \cdot 12 \mathrm{H}_{2} \mathrm{O}$ in the next method ('B'). It is speculated that either less $\mathrm{Na}$ in the precursor, or the presence of water in the synthesis method could influence surface adsorption of $\mathrm{Na}$ ions. Therefore $\mathrm{EtOH}$ and $\mathrm{MeOH}$ were used to facilitate ion exchange, and precursors without water were used (anhydrous $\mathrm{NaH}_{2} \mathrm{PO}_{4}$ and $\mathrm{Na}_{2} \mathrm{HPO}_{4}$ ).

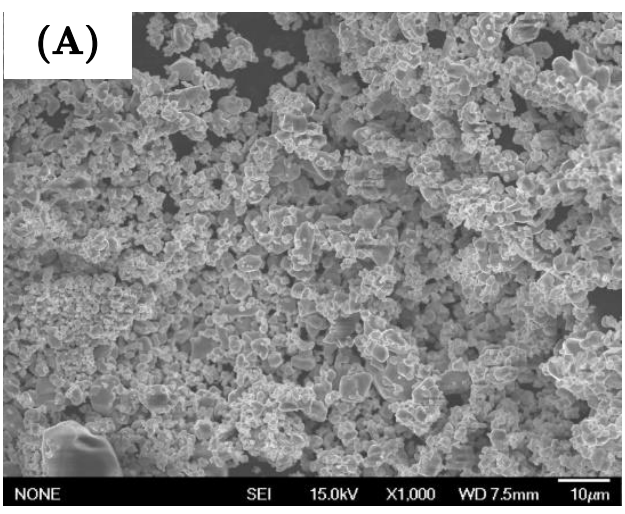

(B)

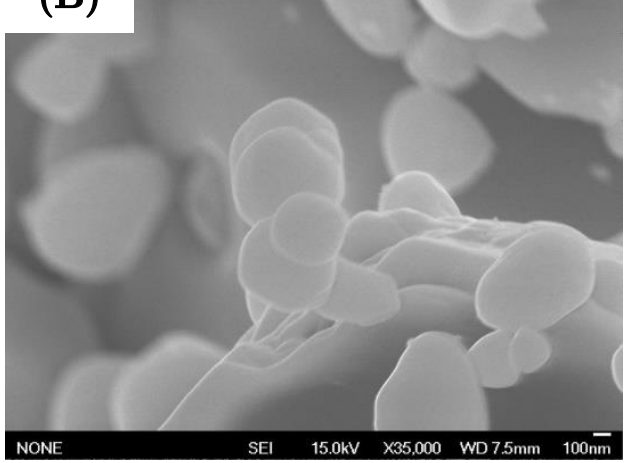



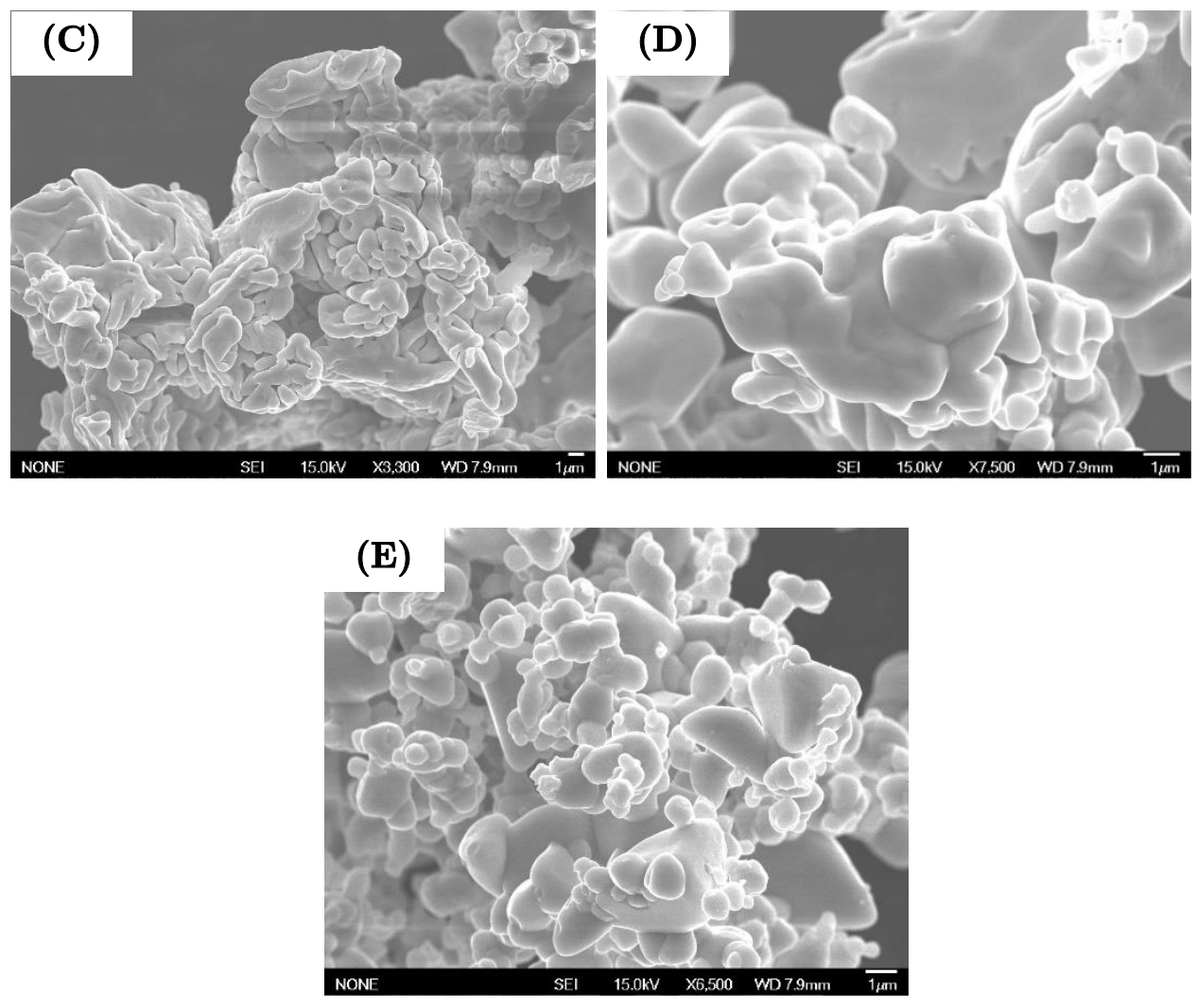

Figure 21. SEM micrographs of samples B1 (A \& B), B2 (C \& D), and B3 (E).

As shown in Figure 21, all B-series samples shown some degree of coagulation/agglomeration, with hugely irregular 'particle' size. With such an obviously large particle size range, it would be impossible to attribute an activity change to a definitive particle size effect. The XRD data (Figure 22) shows that $B 1$ is phase pure, whilst $B 2$ and $B 3$, have unknown subphases, which could not be identified. The UV-Vis absorbance spectra (Figure 23) of the samples show all three samples exhibit an absorption edge typical of silver phosphate. Since there are no other detectable absorption band edges besides $\mathrm{Ag}_{3} \mathrm{PO}_{4}$, it is theorised that the unknown compound is not a semiconductor and therefore does not absorb light in an interband transition process. All three samples display similar BET SSA (Table 5), yet display OERs nearly double that of the samples synthesised using method 'A' - with the exception of B2 (Figure 24). The unknown subphase produced during synthesis could be a result of the precursor, however, the presence of the sub-phase is certainly detrimental to the activity. 


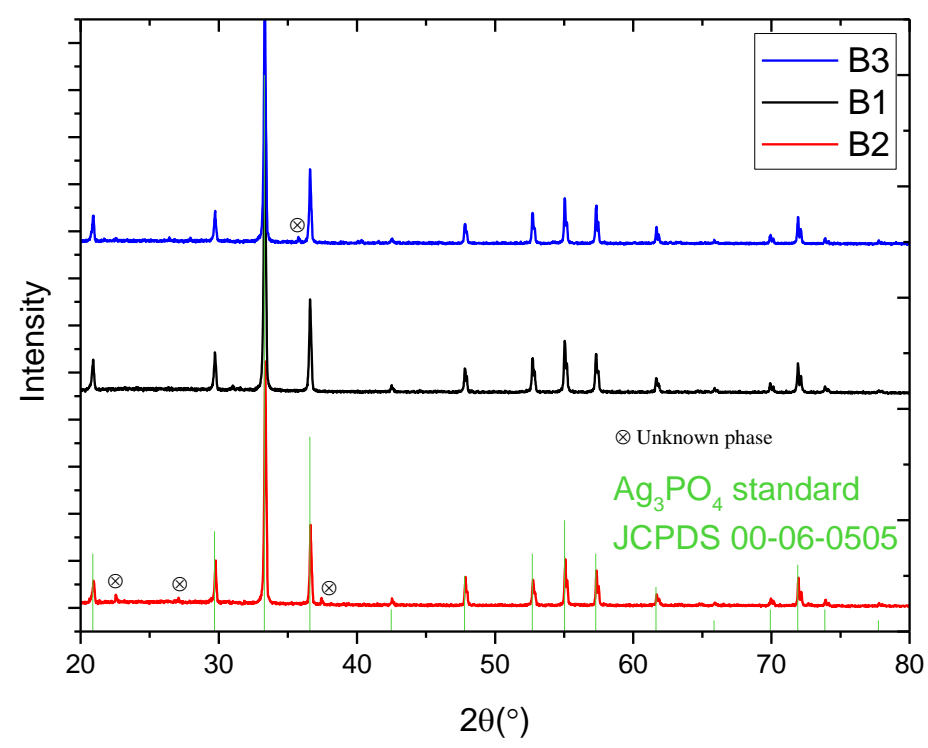

Figure 22. XRD patterns for samples B1, B2 and B3. The standard ISCD/JCPDS pattern for $\mathrm{Ag}_{3} \mathrm{PO}_{4}$ is shown as red bars. The Bragg peaks of the unknown phase are indicated by the symbol ' $\otimes$ '.

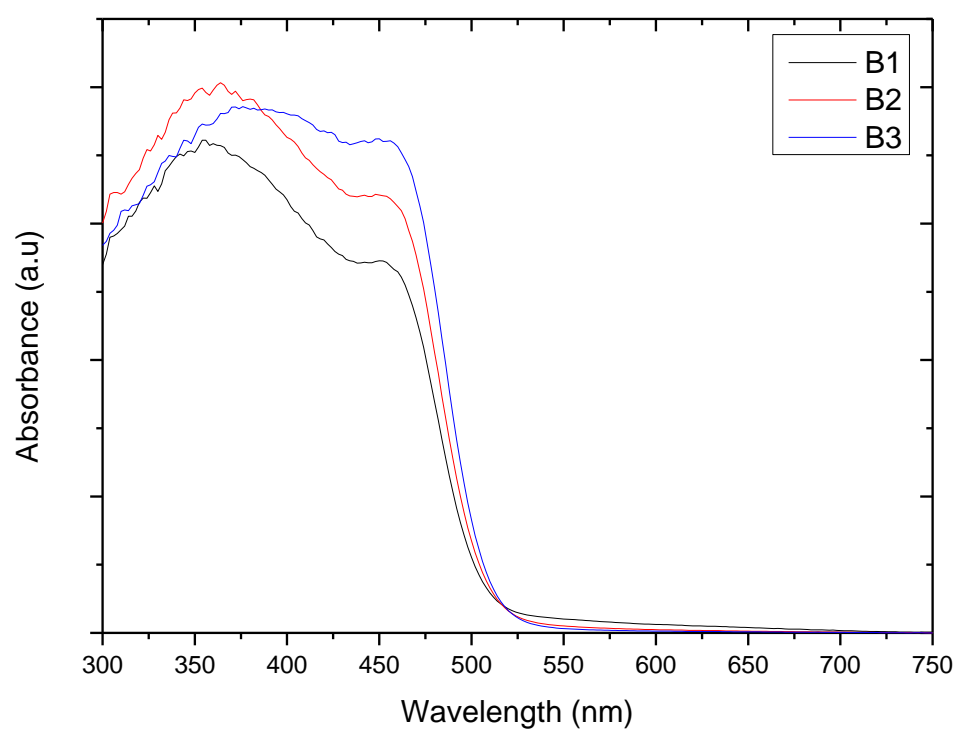

Figure 23. UV-Vis absorbance spectra for samples B1, B2 and B3.

The oxygen evolution rate of both B1 and B3 (after one hour; 1357 and $1159.4 \mu \mathrm{mol} \mathrm{h}{ }^{-1} \mathrm{~g}^{-1}$ ), using $\mathrm{Na}_{2} \mathrm{HPO}_{4}$ as a precursors, and with the addition of a small amount of non-aqueous solvent, is analogous to that reported by Yi et al $\left(1272 \mu \mathrm{mol} \mathrm{h}^{-1} \mathrm{~g}^{-1}\right)$. It appears that the precursor must be anhydrous $\mathrm{Na}_{2} \mathrm{HPO}_{4}$, and be kept anhydrous with the use of an alcohol to keep the 
crystal structure phase pure, and OER high. Despite B1 having a slightly smaller surface area in comparison to B3 (1.01 vs $\left.1.29 \mathrm{~m}^{2} \mathrm{~g}^{-1}\right)$, the OER rate is higher, again suggesting that the activity of $\mathrm{Ag}_{3} \mathrm{PO}_{4}$ is not dramatically influenced by surface area.

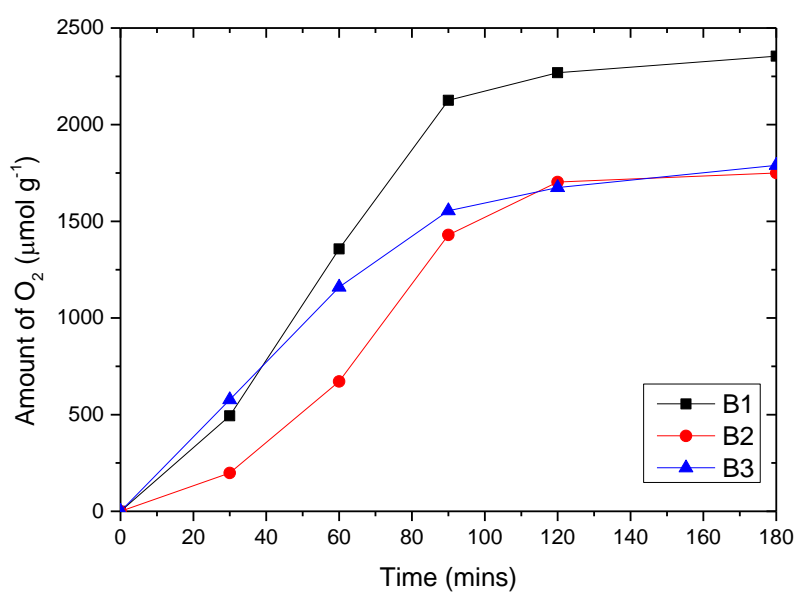

Figure 24. Oxygen evolution under visible light $(\lambda>420 \mathrm{~nm})$ from water, in the presence of $\mathrm{AgNO}_{3}(0.85 \mathrm{~g})$, at neutral $\mathrm{pH}$, using $0.2 \mathrm{~g}$ photocatalyst (B1, B2, B3). 


\subsubsection{Method ' $C$ '}
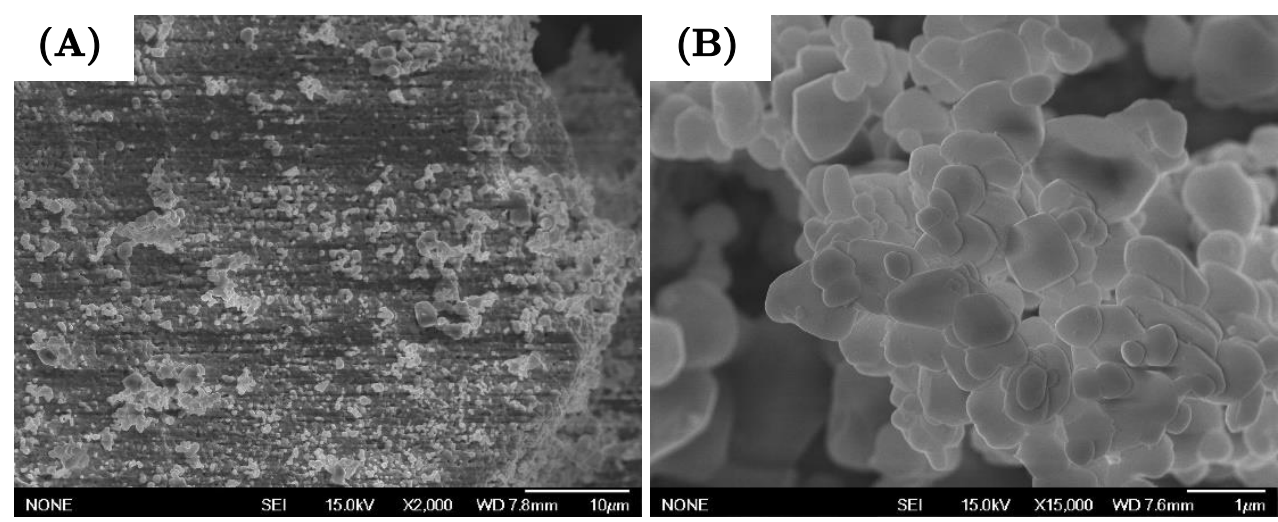

Figure 25. SEM micrographs of sample C1 in low (A) and high (B) resolution.

Both low and high resolution SEM micrographs of samples C1 show that the compound has a similar roughly spherical morphology to that of samples synthesised by the previous two methods, 'A' and 'B' (Figure 25). The compound has an almost identical absorbance spectrum (edge at approximately $506 \mathrm{~nm}$ ) to all previously synthesised samples (Figure 26), and is also pure phase $\mathrm{BCC} \mathrm{Ag}_{3} \mathrm{PO}_{4}$ (Figure 27). However, Figure 28 illustrates that the compound has a higher OER than any other sample, at 1418.75 $\mu \mathrm{mol} \mathrm{h} \mathrm{h}^{-1}$, exceeding that reported by $\mathrm{Yi}$ et al.

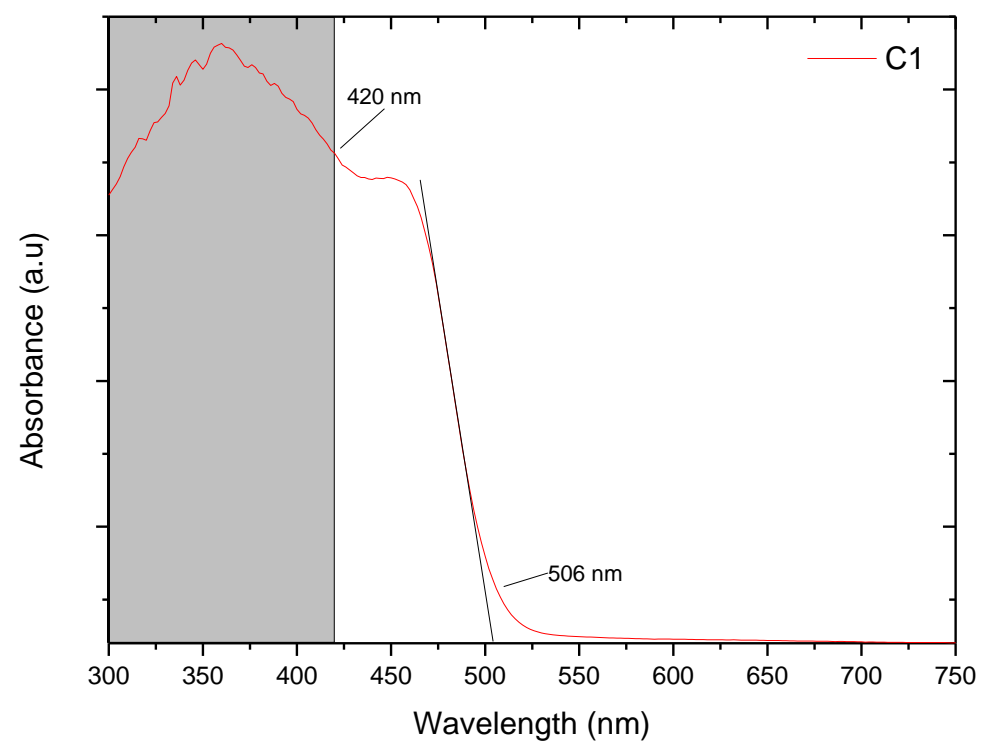

Figure 26. UV-Vis absorbance spectra of sample C1. The grey area visually demonstrates the range of the $420 \mathrm{~nm}$ long pass filter. A black line indicates the band edge and approximate absorption limit. 


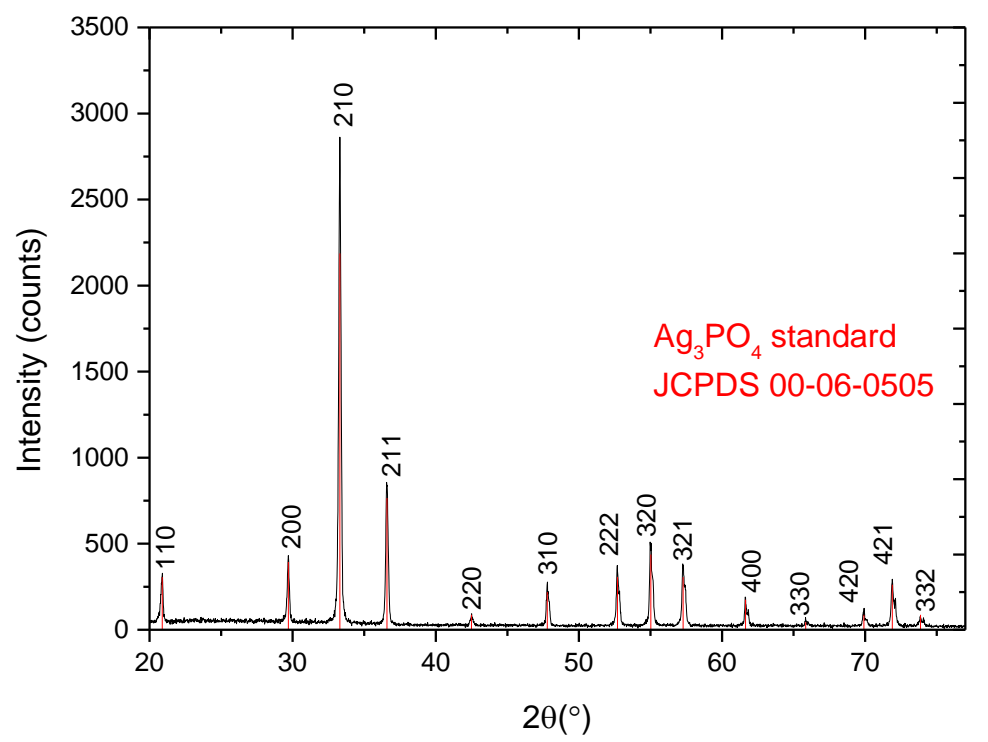

Figure 27. XRD pattern for sample C1. The standard ISCD/JCPDS pattern for $\mathrm{Ag}_{3} \mathrm{PO}_{4}$ is shown as red bars, and the corresponding miller indices of each plane are indicated above each peak.

Whilst it cannot be proven directly, it would appear to be by using a larger volume of ethanol during synthesis, and thereby having a more dilute solution, is beneficial in synthesising a compound which has little or no unreacted species on its surface. What can be said however, is that the BET SSA does not appear to influence the OER on any $\mathrm{Ag}_{3} \mathrm{PO}_{4}$ photocatalyst sample. Sample C1 demonstrates the largest OER, yet has the smallest BET SSA (Table 6). Although no definitive conclusion can be drawn as to an OER determinate for $\mathrm{Ag}_{3} \mathrm{PO}_{4}$, it can be said that the purity of the crystal phase of the compound is paramount, and also that the precursor and solvent used in synthesis is very important. However, it is now possible to synthesise high purity $\mathrm{Ag}_{3} \mathrm{PO}_{4}$ which demonstrates activity for oxygen evolution from water which is comparable with that shown to be possible by $\mathrm{Yi}$ et $\mathrm{al}^{7}$. The slight difference between the active sample (C1) synthesised in this study, and that by Yi et al could be from the different gas chromatographs used, or from the different systems (Yi et al uses a vacuum system, whilst the system in this report is an Argon atmosphere at 1 bar). Much less likely however, dissolved oxygen could still be contained in the system, which has not been flushed out by the argon purging procedure. 


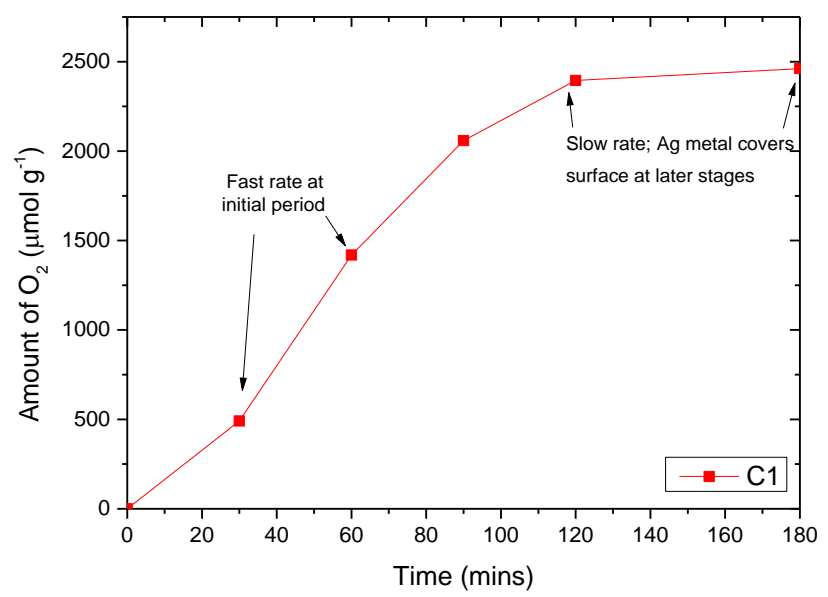

Figure 28. Oxygen evolution under visible light $(\lambda>420 \mathrm{~nm})$ from water, in the presence of $\mathrm{AgNO}_{3}(0.85 \mathrm{~g})$, at neutral $\mathrm{pH}$, using $0.2 \mathrm{~g}$ photocatalyst (C1). Indicated are the regions of most importance; the initial period where $\mathrm{O}_{2}$ evolution rate is high (rates are calculated from this point), and where the rate deviates from linearity in the later stages of the experiment.

\subsubsection{Facet control of $\mathrm{Ag}_{3} \mathrm{PO}_{4}(\operatorname{method} \text { ' } \mathrm{D} \text { ') })^{213}$}

The previous section demonstrated that synthesis parameters of have a significant impact on the OER of $\mathrm{Ag}_{3} \mathrm{PO}_{4}$ irrespective of surface area. As mentioned previously, specific exposing facets often dominate activity of a catalyst, including $\mathrm{TiO}_{2}$ for water splitting and $\mathrm{Ag}_{3} \mathrm{PO}_{4}$ for organic decomposition ${ }^{149,220}$. Therefore collaborators, Naoto Umezawa and Jinhua Ye, performed surface energy calculations of $\{100\},\{110\}$, and $\{111\}$ planes, using a DFT $+U$ approach, and found that out of the three planes, $\{111\}$ possessed the largest surface energy. Despite of the importance of the exposing facets, no data was available for whether or not the exposing facet influenced the photocatalytic rate of oxygen evolution from water, this line of investigation was pursued.

The surface structures generated by the DFT $+\mathrm{U}$ method are based on a slab model including 192 atoms of $\mathrm{Ag}_{3} \mathrm{PO}_{4}$ with $10 \AA$ thickness of a vacuum region. Geometry relaxations were performed with the mid layers of the slab fixed. The relaxed geometries of the three surfaces are depicted in Figure 29. The surface energy $(\gamma)$ was calculated from the following formula:

Equation 35. 


$$
\gamma=\frac{\left(E_{\text {slab }}-N E_{\text {bulk }}\right)}{2 A}
$$

where $E_{\text {slab }}$ is the total energy of the slab and $E_{\text {bulk }}$ is the total energy of the bulk per unit. $\mathrm{N}$ and $\mathrm{A}$ are the number of units included in the slab and the surface area. The obtained surface energies are $0.67 \mathrm{~J} / \mathrm{m}^{2}, 0.78 \mathrm{~J} / \mathrm{m}^{2}$, and $1.65 \mathrm{~J} / \mathrm{m}^{2}$ for $\{100\},\{110\}$, and $\{111\}$ respectively.

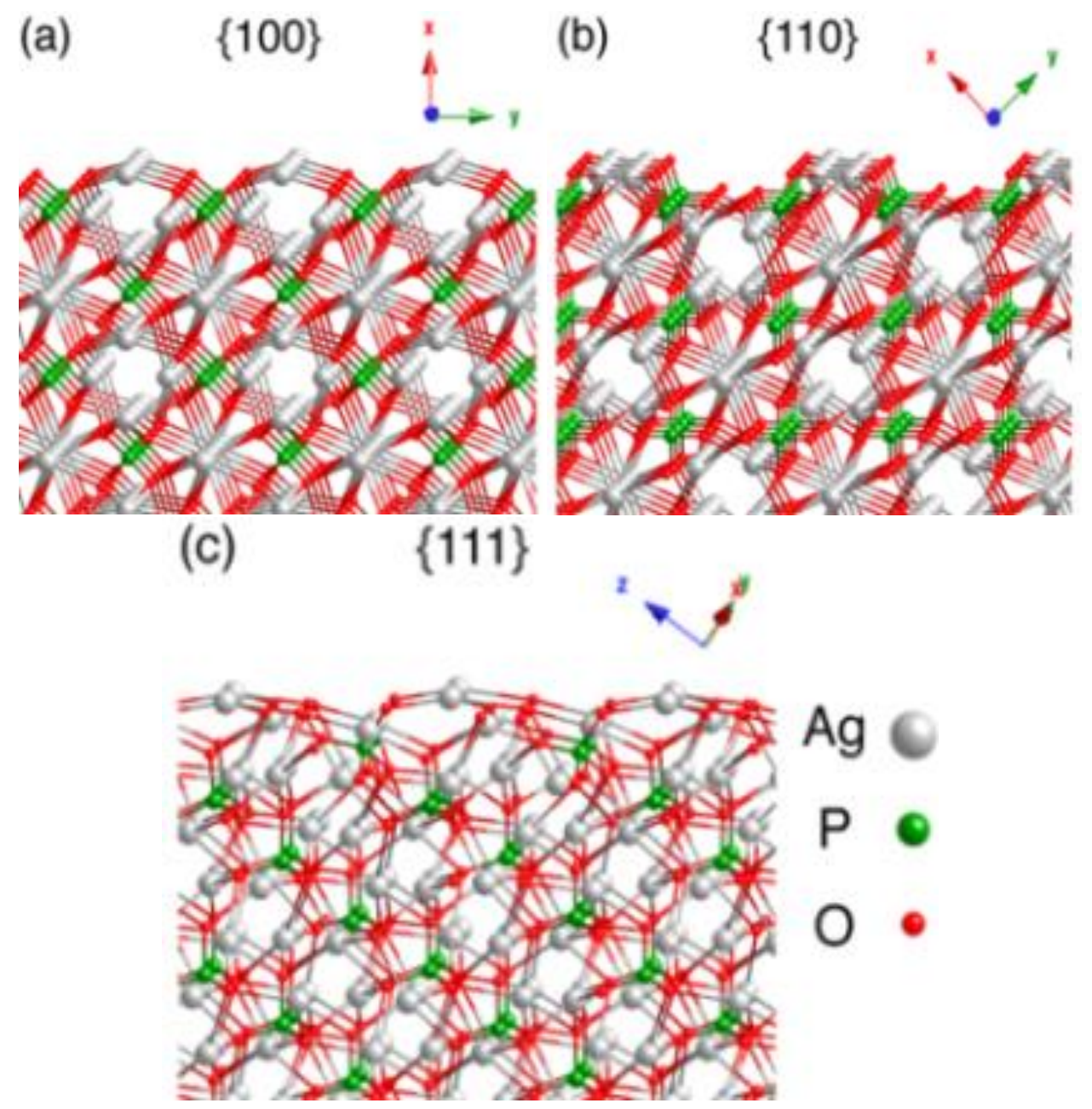

Figure 29. Relaxed geometries for (a) $\{100\}$, (b) $\{110\}$, and (c) $\{111\}$ surface of $\mathrm{Ag}_{3} \mathrm{PO}_{4}$ (calculations performed by Naoto Umezawa, NIMS) ${ }^{213}$. 
Table 6. Properties of different $\mathrm{Ag}_{3} \mathrm{PO}_{4}$ crystals.

\begin{tabular}{|c|c|c|c|c|c|c|}
\hline Sample & Facet & $\begin{array}{c}\text { Surface } \\
\text { Energy } \\
\left(\mathrm{J} \mathrm{m}^{-2}\right)\end{array}$ & $\begin{array}{c}\text { Hole } \\
\text { mass } \\
\left(\mathrm{m}_{\mathrm{h}}{ }^{*}\right)\end{array}$ & $\begin{array}{c}\text { Surface } \\
\text { Area } \\
\left(\mathrm{m}^{2} \mathrm{~g}^{-1}\right)\end{array}$ & $\begin{array}{c}\text { Initial } \mathrm{O}_{2} \\
\text { evolution } \\
\text { rate }(\mu \mathrm{mol} \\
\left.\mathrm{h}^{-1} \mathrm{~g}^{-1}\right)\end{array}$ & $\begin{array}{c}\text { TOF } \\
\mathrm{min}^{-1}\end{array}$ \\
\hline Tetrahedron & 111 & 1.67 & 1.53 & 1.0 & 6074 & 2.60 \\
\hline $\begin{array}{c}\text { Cube } \\
\text { Rhombic } \\
\text { dodecahedron }\end{array}$ & 110 & 0.78 & 2.08 & 2.4 & 426 & 0.17 \\
\hline
\end{tabular}

The results clearly show that the $\{111\}$ facet is over two times more energetic than the second highest facet, $\{110\}$. Previous studies by Umezawa et al about $\mathrm{Ag}_{3} \mathrm{PO}_{4}$ also show an anisotropic distribution of electron-hole masses $\left(\mathrm{m}_{\mathrm{h}}{ }^{*} \text {, Table } 6\right)^{148}$. According to earlier reports, smaller values of $\mathrm{m}_{\mathrm{h}}{ }^{*}$ lead to a greater hole mobility; therefore making transport to the surface much more straightforward, and thus decreasing the probability of recombination ${ }^{221}$. Tetrahedral $\mathrm{Ag}_{3} \mathrm{PO}_{4}$ crystals purely composed of $\{111\}$ facets were shown to be theoretically possible using Materials Studio (Figure 30). 

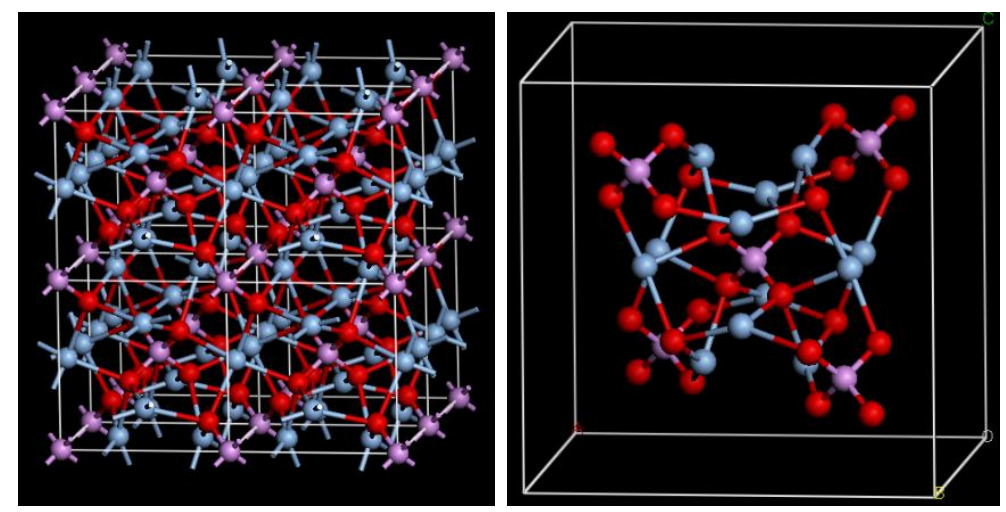

Figure 30. (A) $\mathrm{Ag}_{3} \mathrm{PO}_{4} 2 \times 2 \times 2$ supercell and (B) $\mathrm{Ag}_{3} \mathrm{PO}_{4}$ tetrahedron cell (Figure from Martin et $\mathrm{al}^{213}$ ).

A kinetically controlled approach in the absence of surfactants operated at ambient temperature was employed (Method 'D'), and crystals with tetrahedral morphology and preferred $\{111\}$ facets were fabricated in high yield (Figure 32). The exposing $\{111\}$ facets were controlled by manipulating the concentration of $\mathrm{H}_{3} \mathrm{PO}_{4}$ in ethanol; whereby excess amounts of phosphoric acid not only provide a precursor, but also control the rate of $\mathrm{Ag}_{3} \mathrm{PO}_{4}$ nucleation and initial growth (seed formation). As the $\mathrm{H}_{3} \mathrm{PO}_{4}$ volume is increased from $2 \mathrm{~cm}^{3}$ to $15 \mathrm{~cm}^{3}, \mathrm{Ag}_{3} \mathrm{PO}_{4}$ tetrapods grow into tetrahedrons through a most probable Oswald ripening effect, as more $\mathrm{H}_{3} \mathrm{PO}_{4}$ is available at larger concentrations, and thus smaller particles/precursors will eventually form tetrahedrons, from tetrapods. Directional growth is halted at a specific concentration of $\mathrm{H}_{3} \mathrm{PO}_{4}$ in comparison to the silver precursor $\left(\mathrm{AgNO}_{3}\right)$. No other sub-structures were found either within the powdered samples, or on the clean surfaces of the particles.

Using method 'C', mixed faceted shapes are formed as previously reported ${ }^{7}$. Rhombic dodecahedrons $\{110\}$ and cubes $\{100\}$, which have been reported for organic dye photodecomposition ${ }^{149}$, were also fabricated to explore the correlation of facets with photocatalytic activities for oxygen production from water. The different morphologies were also been confirmed by SEM micrographs (Figure 31). In order to further verify the tetrahedral morphology, TEM tilt studies have carefully been undertaken. As seen in Figure 33, the faceted compound is rotated on axis, to reveal 4 identical facets in total from different angles; proving they are tetrahedrons with $\{111\}$ surfaces. 

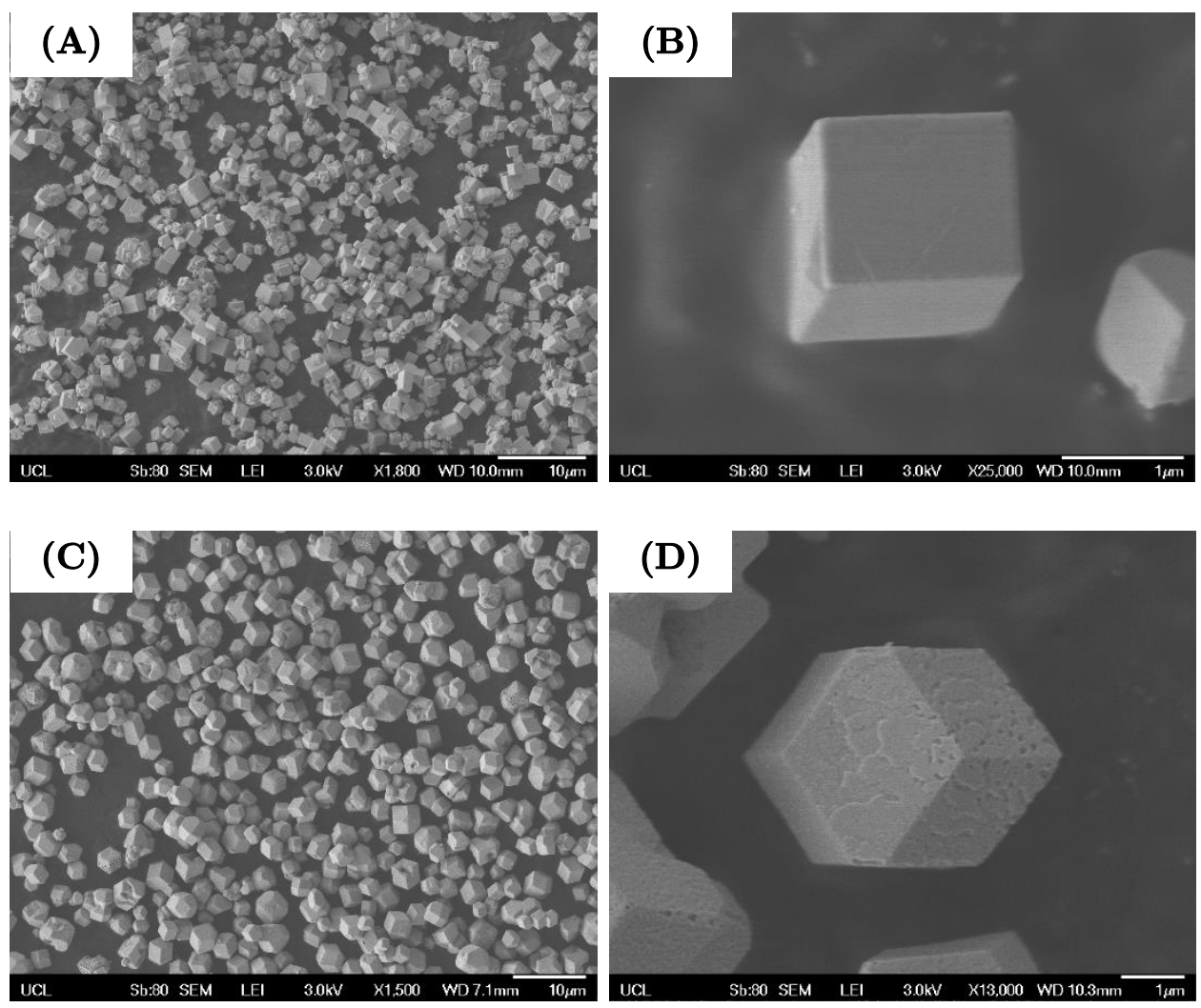

(D)

Figure 31. SEM micrographs of silver phosphate cubic crystals (A and B), and rhombic dodecahedral crystals (C and D). 

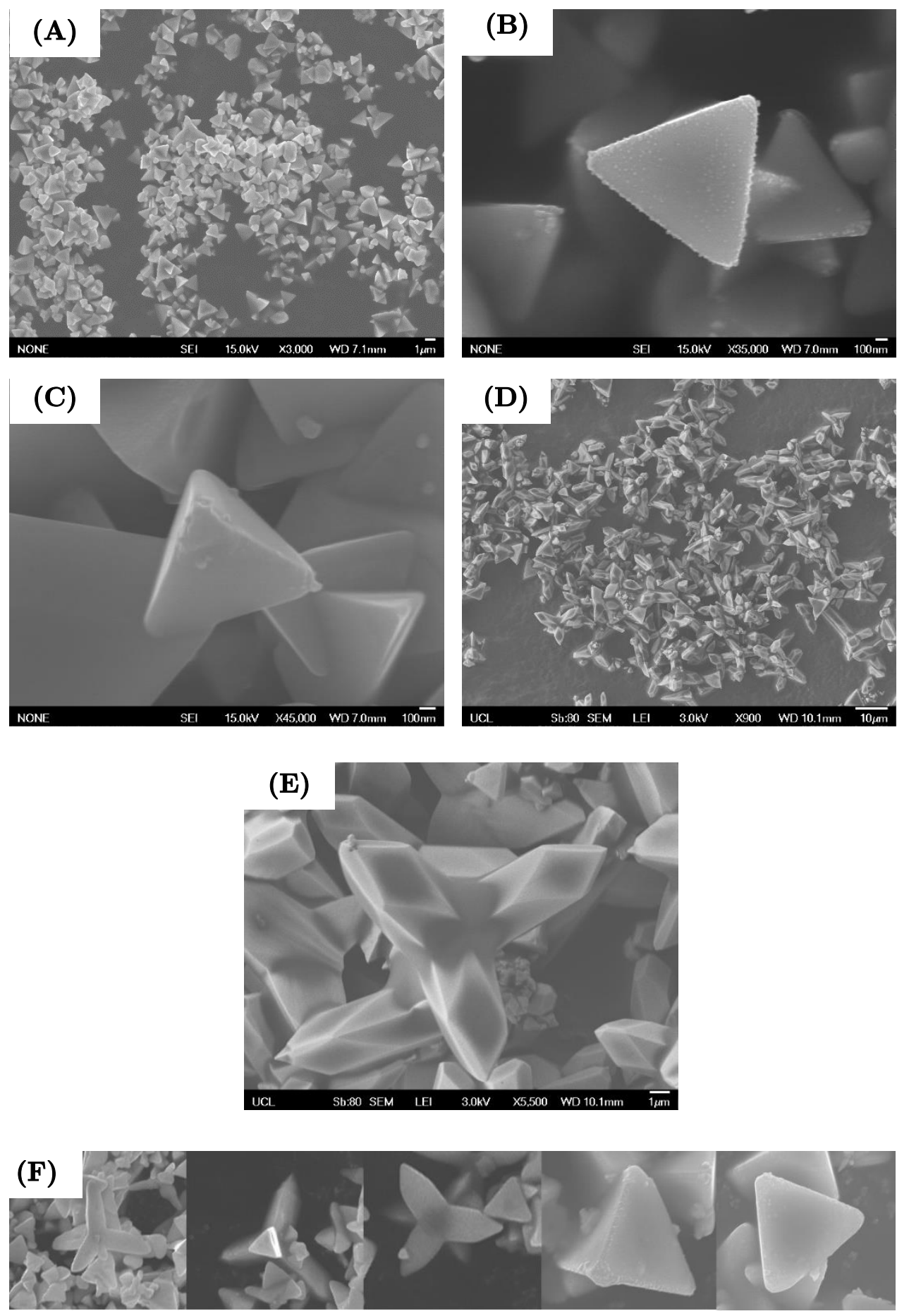

Figure 32. Low (A) and high (B, C) magnification SEM micrographs of $\mathrm{Ag}_{3} \mathrm{PO}_{4}$ tetrahedrons $\left(20 \mathrm{~cm}^{3} \mathrm{H}_{3} \mathrm{PO}_{4}\right)$. Low (D) and high (E) magnification SEM micrographs of $\mathrm{Ag}_{3} \mathrm{PO}_{4}$ tetrapods synthesised using $2 \mathrm{~cm}^{3} \mathrm{H}_{3} \mathrm{PO}_{4}$. (F) SEM micrographs illustrating the formation of $\mathrm{Ag}_{3} \mathrm{PO}_{4}$ tetrahedrons from 
tetrapods by varying the concentration of $\mathrm{H}_{3} \mathrm{PO}_{4}$ (left to right; $2,5,10,15$, $20 \mathrm{~cm}^{3} \mathrm{H}_{3} \mathrm{PO}_{4}$ )

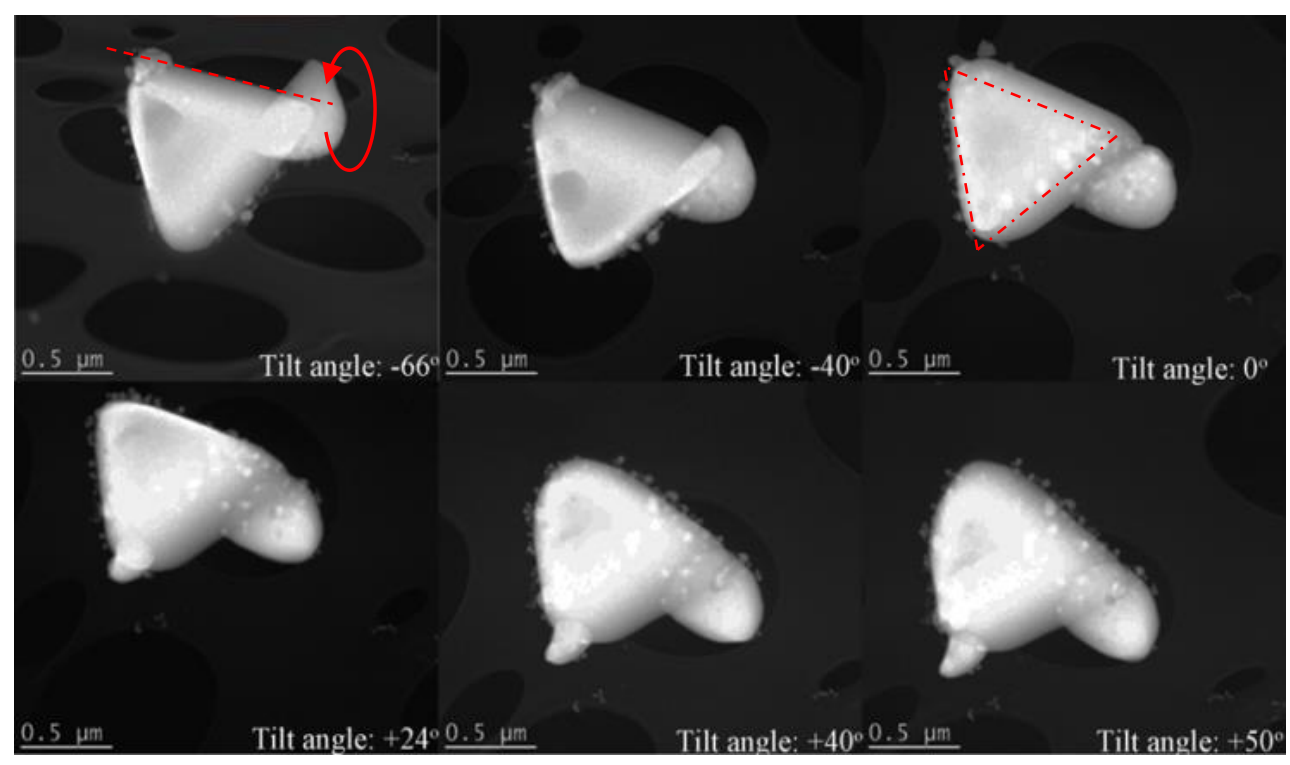

Figure 33. TEM micrograph tilt study, performed by collaborator, Xiaowei Chen. A tetrahedron indicated by dotted lines is rotated on axis from -66 to $+50^{\circ}$.

The X-Ray Diffraction patterns of the three samples (Figure 34) indicate a full phase match to that of body-centred cubic crystalline $\mathrm{Ag}_{3} \mathrm{PO}_{4}$ (6.004 Å, BCC, P-43n, JCPDS no.06-0505), and show no other trace peaks associated with the precursors used in fabrication, indicating that the synthesis methods lead to a completely well crystallised pure phase compound. The diffraction patterns exhibit stark variations in intensity ratios. Tetrahedral $\mathrm{Ag}_{3} \mathrm{PO}_{4}$ particles have an intensity ratio of 0.98 between (222) and (200) planes, whilst the rhombic dodecahedron and cubic structures have an approximate ratio of 0.68 , confirming that tetrahedral particles are comprised of $\{111\}$ crystalline planes ${ }^{222}$. The intensity ratio between (110) and (200) for rhombic dodecahedrons is 1.23 , and 0.57 for the cubic crystals, confirming as previously reported that rhombic dodecahedrons and cubic structures are composed of $\{110\}$ and $\{100\}$ facets respectively ${ }^{223}$. It is noted that the (111) Bragg peak is not directly seen due to the reflection conditions of the space group, similarly, (100) cannot be seen either. 


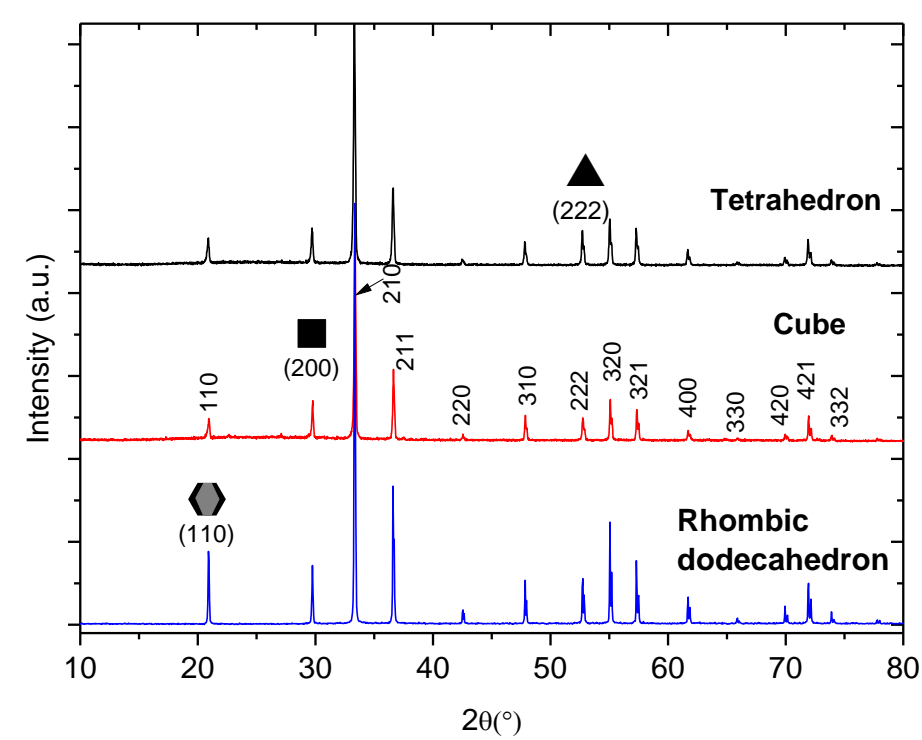

Figure 34. XRD pattern of faceted $\mathrm{Ag}_{3} \mathrm{PO}_{4}$ crystals.

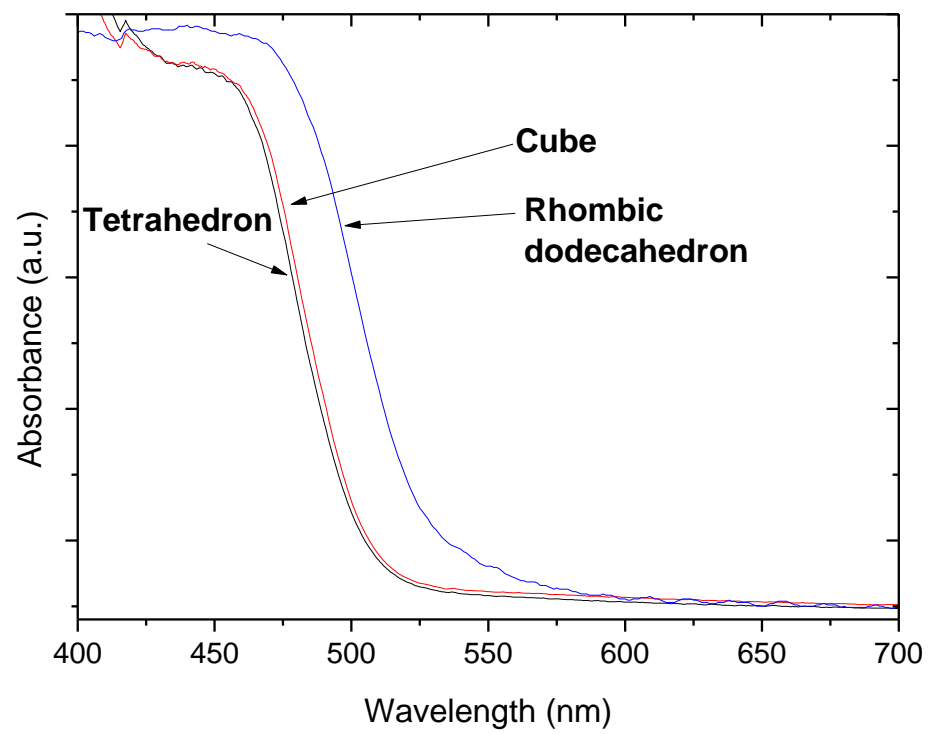

Figure 35. UV-Vis absorbance spectra of faceted $\mathrm{Ag}_{3} \mathrm{PO}_{4}$ crystals.

Before testing the catalytic activity for direct water photooxidation, the UV-Vis absorption spectra between the three different morphologies were measured (Figure 35). There is a large difference between the samples; rhombic dodecahedrons are able to absorb light up to approximately 530 $\mathrm{nm}$, whilst both tetrahedrons and cubes have an absorption edge around $515 \mathrm{~nm}$. Since the size of the crystals is similar (see Table 7 \& Figure 36) and there are no signs of foreign elemental doping in the sample, it is thought that the absorption edge shift is likely to be due to the faceted morphology. It is also possible that the absorbance shift is due to a particle 
quantum size effect (continuous to discrete band shift due to fewer energy levels). However this is unlikely as the particles are over $1 \mu \mathrm{m}$ diameter.

Table 7. Size, range, and standard deviation (S.D.) of various $\mathrm{Ag}_{3} \mathrm{PO}_{4}$ crystals. Mean, mode, range, and S.D. is calculated for 100 particles using ImageJ.

\begin{tabular}{|c|c|c|c|c|}
\hline Morphology & $\begin{array}{c}\text { Mean particle } \\
\text { size }(\mu \mathrm{m})\end{array}$ & $\begin{array}{c}\text { Modal } \\
\text { particle } \\
\text { size }(\mu \mathrm{m})\end{array}$ & $\begin{array}{c}\text { Size range } \\
(\mu \mathrm{m})\end{array}$ & $\begin{array}{c}\text { Standard } \\
\text { deviation }\end{array}$ \\
\hline Tetrahedron & 1.24 & 1.09 & $0.70-2.07$ & 0.34 \\
\hline Cube & 1.21 & 1.02 & $0.51-2.13$ & 0.33 \\
\hline $\begin{array}{c}\text { Rhombic } \\
\text { dodecahedron }\end{array}$ & 2.91 & 2.79 & $1.53-4.40$ & 0.65 \\
\hline
\end{tabular}

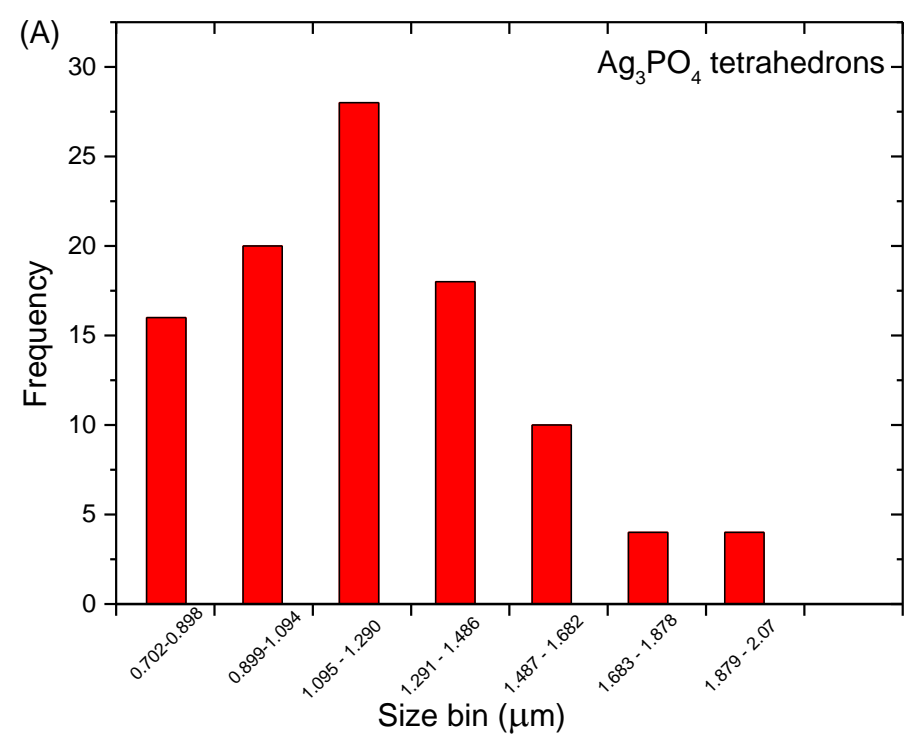



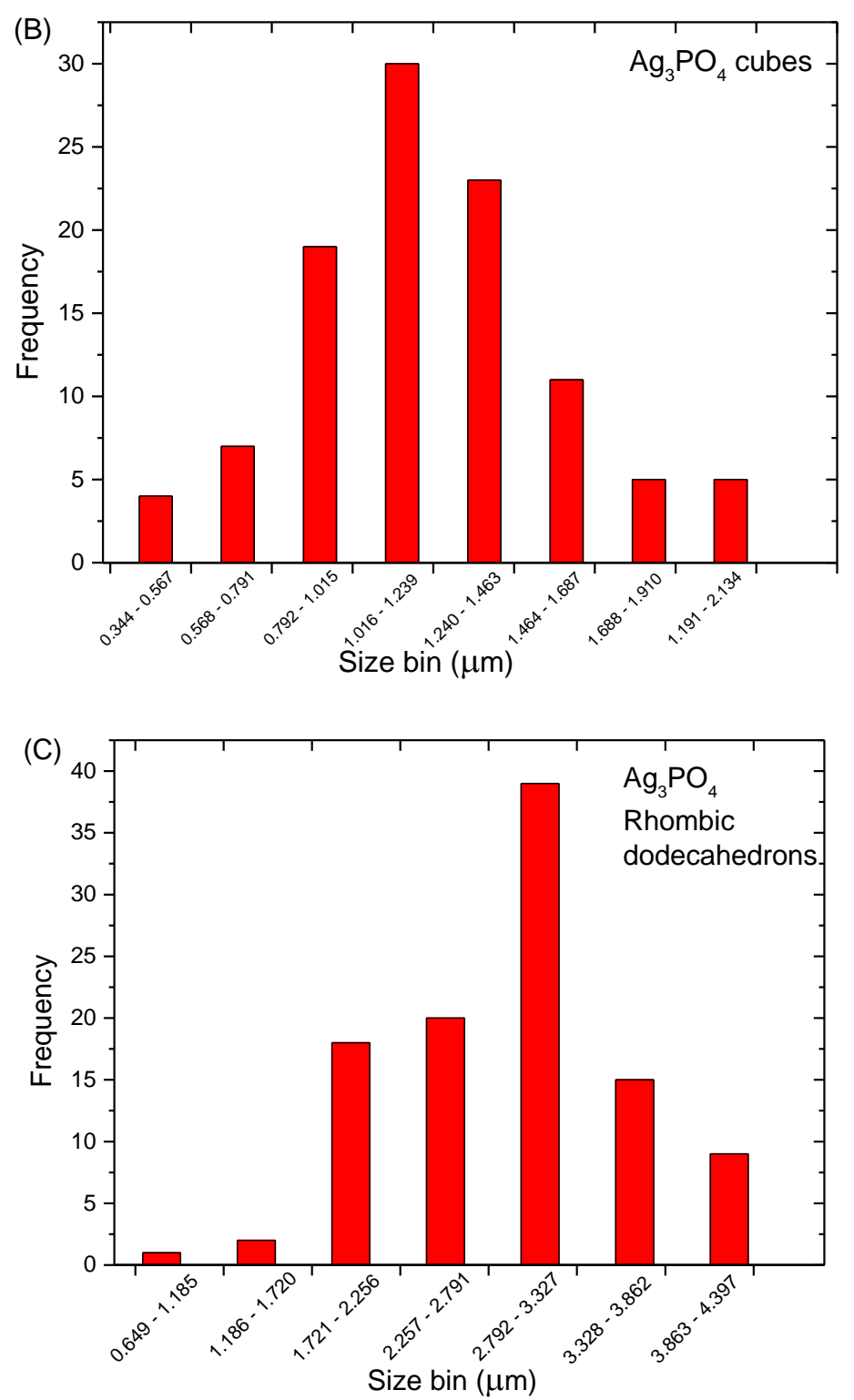

Figure 36. Particle diameter histograms for $\mathrm{Ag}_{3} \mathrm{PO}_{4}$ (A) tetrahedrons, (B) cubes, (C) rhombic dodecahedrons.

The faceted samples were tested for the photooxidation of water in the presence of an electron scavenger under ambient conditions. As seen in Figure 37 (A), oxygen evolves extremely fast, at an initial rate of $6072 \mu \mathrm{mol}$ $\mathrm{hr}^{-1} \mathrm{~g}^{-1}$ for tetrahedral $\mathrm{Ag}_{3} \mathrm{PO}_{4}$ particles under full arc irradiation, over 10 times more than either rhombic dodecahedral or cubic samples which have been reported for organic photodecomposition but not water splitting ${ }^{149}$. Furthermore, the benchmark material for visible light water photooxidation, $\mathrm{BiVO}_{4}$, was prepared using a previously described 
method $^{17}$. Tests under visible light for mixed faceted samples (prepared by method ' $C$ ') show a very similar oxygen evolution rate as reported (Figure $37(\mathrm{~B})) .\{111\}$ faceted $\mathrm{Ag}_{3} \mathrm{PO}_{4}$ evolves oxygen at a rate of 6.5 times $\mathrm{BiVO}_{4}$ as shown in Figure $37(B)$. The $\{111\}$ faceted sample shows $242 \%$ more activity in comparison to a mixed faceted sample synthesised using method ' $\mathrm{C}$ ' and reported by $\mathrm{Yi}$ et $\mathrm{al}^{7}$ (Figure 37). The activity of all samples was also compared under visible light irradiation $(\lambda>420 \mathrm{~nm}$, Figure $37(C))$. Both rhombic dodecahedrons and cubic crystals are less active than the mixed faceted $\mathrm{Ag}_{3} \mathrm{PO}_{4}$ for water photooxidation, with tetrahedral $\mathrm{Ag}_{3} \mathrm{PO}_{4}$ performing best under visible light irradiation.
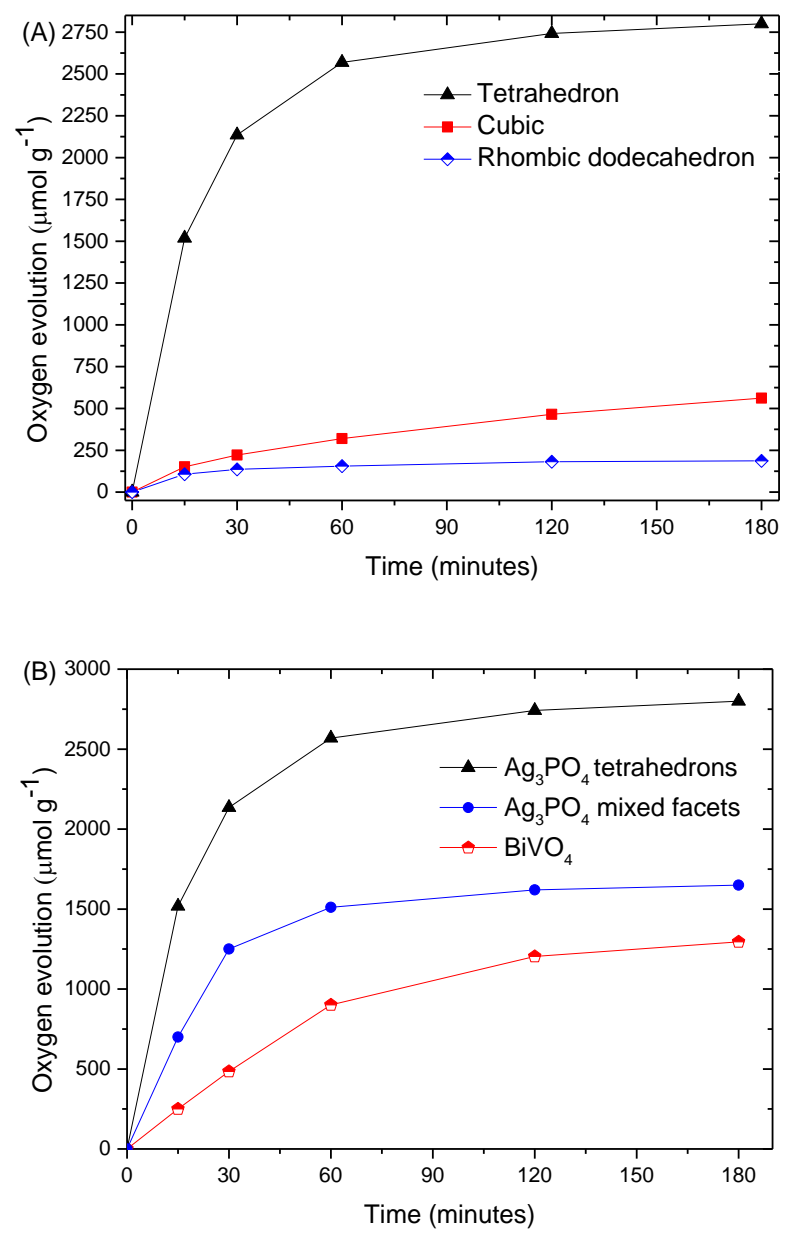


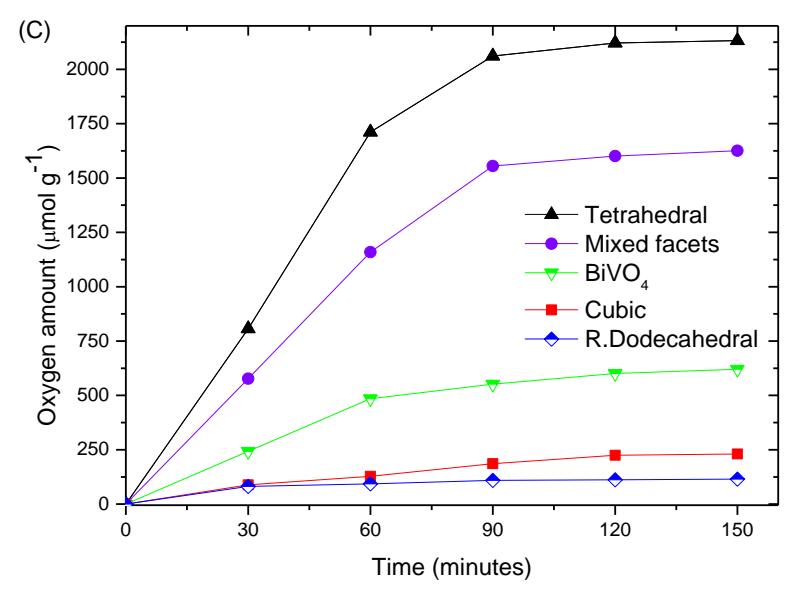

Figure 37. (A) Oxygen yield comparison of $\mathrm{Ag}_{3} \mathrm{PO}_{4}$ facets using a $300 \mathrm{~W}$ Xe light source under full arc irradiation, with $\mathrm{AgNO}_{3}$ acting as an electron scavenger. (B) Oxygen yield comparison between tetrahedral $\mathrm{Ag}_{3} \mathrm{PO}_{4}$, previously reported random mixed faceted $\mathrm{Ag}_{3} \mathrm{PO}_{4}$ and $\mathrm{BiVO}_{4}$ under $300 \mathrm{~W}$ Xe lamp full arc irradiation, using $\mathrm{AgNO}_{3}$ as an electron scavenger. (C) Oxygen evolution of tetrahedral crystals using a $300 \mathrm{~W}$ Xe lamp fitted with $420 \mathrm{~nm}$ long pass filter, $\mathrm{AgNO}_{3}$ was used as an electron scavenger $(0.85 \mathrm{~g})$. The mixed facet sample was synthesised using method $\mathrm{C}$ and $\mathrm{Na}_{2} \mathrm{HPO}_{4}$. $\mathrm{BiVO}_{4}$ was synthesised according to a previously reported recipe ${ }^{17}$.



Figure 38. BET adsorption isotherms for $\mathrm{Ag}_{3} \mathrm{PO}_{4}$ tetrahedrons, cubes, and rhombic dodecahedrons.

Using the BET method, tetrahedral $\mathrm{Ag}_{3} \mathrm{PO}_{4}$ crystals were experimentally verified to have a surface area of $1.4 \mathrm{~m}^{2} \mathrm{~g}^{-1}$, somewhat smaller than cubes $\left(1.8 \mathrm{~m}^{2} \mathrm{~g}^{-1}\right)$ and rhombic dodecahedron $\left(2.4 \mathrm{~m}^{2} \mathrm{~g}^{-1}\right)$, where the raw data is shown in Figure 38. All isotherms can be classified as type II, demonstrating 
a plateau at $\mathrm{P} / \mathrm{P}_{0}<0.3$ and no hysteresis; evident of low surface area and very little porosity. The order of activity of oxygen evolution is; tetrahedrons, cubes, rhombic dodecahedrons. Tetrahedral and cubic $\mathrm{Ag}_{3} \mathrm{PO}_{4}$ have a similar particle size, both in terms of mean and mode, and a similar standard deviation. However, the rhombic dodecahedral crystals possess a particle size more than double that of tetrahedral or cubic crystals, and a large variation in size $(2.91 \pm 0.65 \mu \mathrm{m})$. Despite the larger particle size of the rhombic dodecahedron sample, it possesses a larger surface area, attributed to an increased porosity (see Figure 31 (D) for evidence of pores), and can absorb more light. Therefore there is no direct correlation between the oxygen evolution rate and either light absorbance, particle size, or surface area.

In order to make a more reliable and reproducible assessment of the activity of $\{111\}$ faceted crystals, they were also prepared and tested again by me in NIMS, Japan using the same reaction conditions. The difference in activity is approximately $5.1 \%$, which likely arises from the difference in pressure between the two systems. Therefore not only is the synthesis reproducible, but the performance is very similar in different systems as well.

As shown in Table 6, the turnover frequency, which was calculated using Equation 9, divided by time, (over a three hour experiment) is dramatically different between samples, with the largest gulf between tetrahedral and rhombic dodecahedral samples (15 times higher). It should be noted that the TOF min $^{-1}$ is greatly underestimated, as this calculation assumes all atoms are on the surface and involved in surface reactions, which is not true in this particular case, since the particle size is about $2 \mu \mathrm{m}$, as many atoms do not take part in the photocatalytic surface reaction. The turnover number is ca. 5 for tetrahedral $\mathrm{Ag}_{3} \mathrm{PO}_{4}$ particles, meaning that the reaction is a truly photocatalytic process for the $\{111\}$ faceted sample (TON $\geq 1$ is indicative of a catalytic process). Due to the electron scavenger used in the experiments, after a period of around 120 minutes, silver metal $\left(\mathrm{Ag}^{+}+\mathrm{e}^{-}\right.$ $\rightarrow \mathrm{Ag}^{0}$ ) has completely covered the surface of the semiconductor, and significantly prevents further oxidation by blocking incident photon flux, as seen previously, and in other studies $^{34}$, which limits the calculated minimum TOF min $^{-1}$ and turnover number. After the experimental run, the sample was characterised again by XRD. The diffraction pattern of $\mathrm{Ag}_{3} \mathrm{PO}_{4}$ even after an extensive TOF $\mathrm{min}^{-1}$ measurement is very similar to the 
freshly synthesised sample, and as expected, metallic silver was also detected as a dominant surface species (Figure 39).

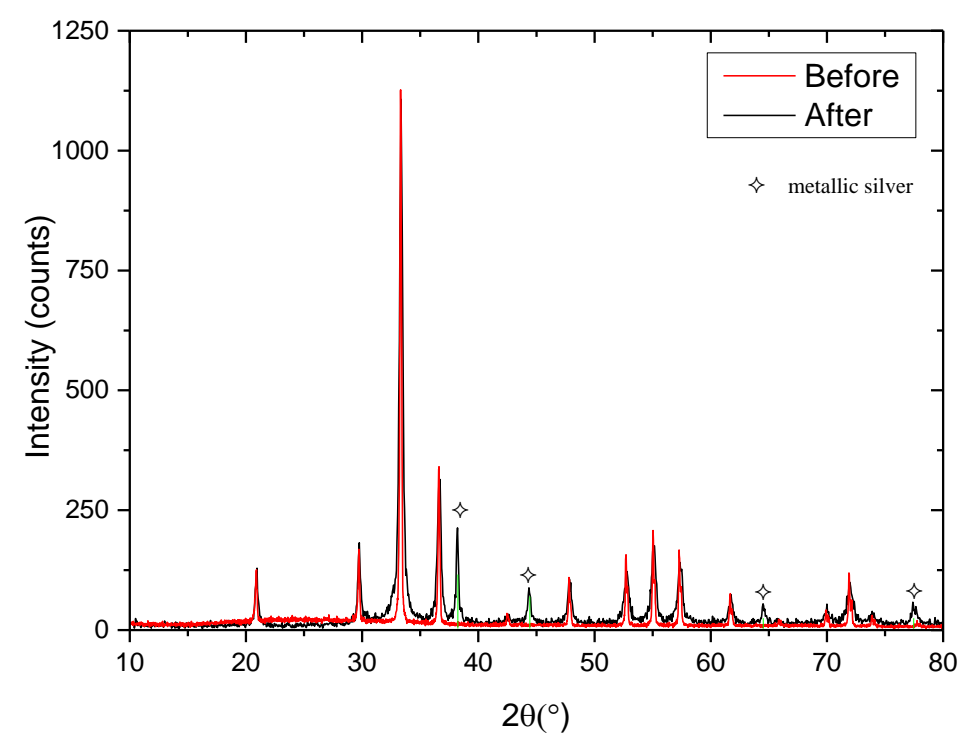

Figure 39. XRD pattern before and after a full arc photocatalytic test for the photooxidation of water by tetrahedral $\mathrm{Ag}_{3} \mathrm{PO}_{4} \cdot \mathrm{AgNO}_{3}$ was used as a scavenger, and thus Ag metal deposited on the surface (JCPDS 00-040783).

The internal quantum yield for water photooxidation was measured to further demonstrate the efficiency of the tetrahedral $\mathrm{Ag}_{3} \mathrm{PO}_{4}$ particles. A high quantum yield was observed; $95 \%$ at $420 \mathrm{~nm}$ and nearly unity at 400 nm (Figure 40). More importantly, the tetrahedral particles show high quantum yield $(>80 \%)$ at a wide wavelength range; from the UV portion of the spectrum throughout the visible until ca. $500 \mathrm{~nm}$, which nearly matches the peak light intensity of solar spectrum (Figure 40 (B) inset). Taking into account the light scattering by the large particles which was assumed zero in our measurement, the actual quantum yield could be higher than the reported. This suggests the enhanced photocatalyst is able to harvest light effectively, separate and then utilise charge carriers extremely efficiently. 

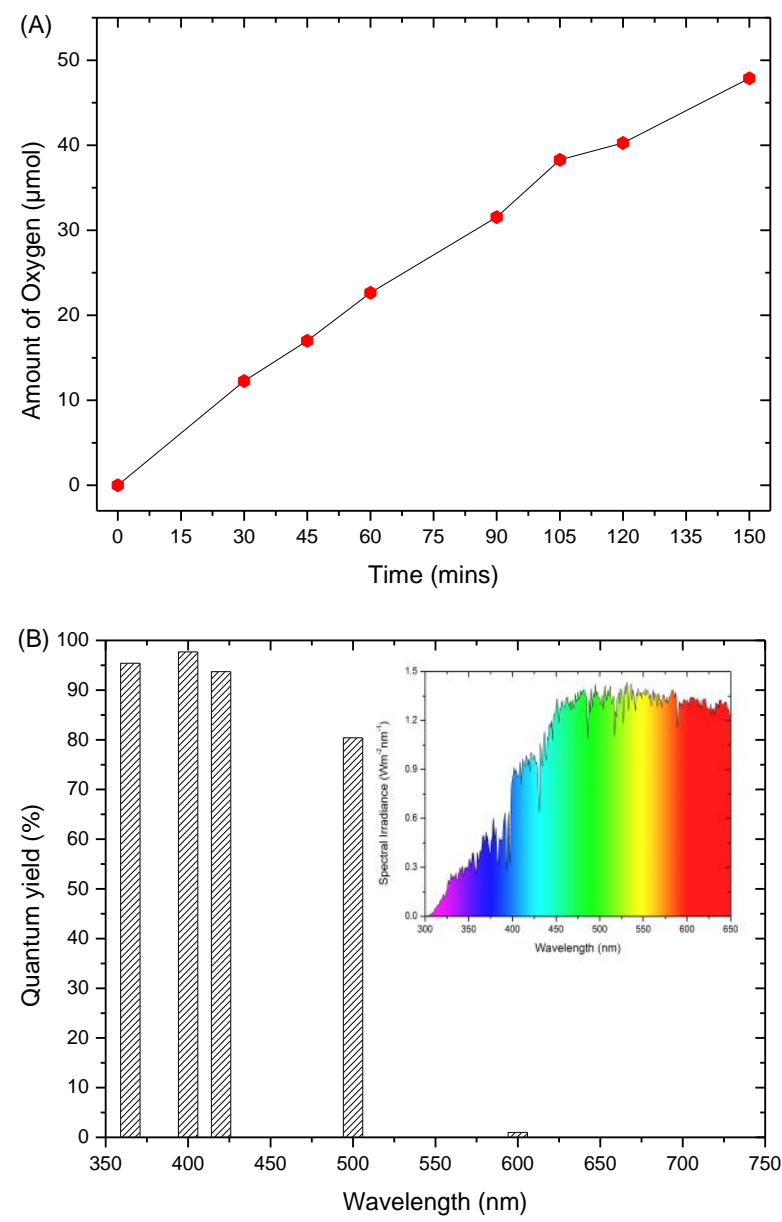

Figure 40. (A) Oxygen evolution using a $400 \mathrm{~nm}$ band pass filter, and (B) Internal quantum yield variation using different band pass filters, using $\mathrm{AgNO}_{3}$ as a scavenger, and a $300 \mathrm{~W}$ Xe light source.

A suggested mechanism for enhanced photocatalytic activity on the faceted compounds states that depending on the surface structure, electrons or holes will specifically migrate to separate $\operatorname{sites}^{224}$. This is due to the differences in the effective masses of holes and electrons, which are directionally sensitive to the crystal structure. The hole mass along the [111] direction is much smaller compared to other directions in $\mathrm{Ag}_{3} \mathrm{PO}_{4}$ crystals (Table 6), indicating that hole mobility and migration towards \{111\} facets is greatly encouraged, which is a critical issue in an n-type semiconductor such as silver phosphate in which holes are the minor species. The water splitting process is dominated by water oxidation as mentioned in the introduction, and thus a higher mobility of holes favours a faster and more efficient transport of holes to the surface for oxidation 
reactions. The reason for the high surface energy of the $\{111\}$ surface is the over-abundance of dangling phosphorus-oxygen bonds, which act as oxidation sites. According to calculations performed by Umezawa, in order to reveal the $\{111\}$ plane, P-O bonds must be cut, resulting in very short layer by layer distances along [111]. From extensive studies in catalysis ${ }^{225}$, it is widely accepted that a high surface energy is very beneficial to surface reaction rates. The high surface energy dramatically enhances the catalytic ability at the surface of $\{111\}$ facets for example, due to more step sites and kinks. The combination of both a high hole mobility and high surface energy results in a unique synergistic effect, culminating in a significant increase in water photooxidation. Both $\mathrm{Ag}_{3} \mathrm{PO}_{4}$ rhombic dodecahedrons and cubes possess slightly higher surface areas, but they have larger hole masses and much smaller surface energies, which leads to a lower activity. It has been reported that both rhombic dodecahedrons and $\mathrm{Ag}_{3} \mathrm{PO}_{4}$ cubes show better activity than the mixed faceted $\mathrm{Ag}_{3} \mathrm{PO}_{4}$ for organic dye photobleaching ${ }^{149}$. This chemical process is mechanistically quite different from water splitting. Thermodynamically, there is a difference in required chemical potentials between water splitting and dye bleaching. More importantly, kinetically as a dominating step in water splitting, water photooxidation requires 4 holes, and occurs on relatively long timescale of ca. 0.5 seconds $^{30,215}$. However the dominating step in organic photooxidation is oxygen reduction reaction, which is relatively fast on microsecond timescale ${ }^{30,42,215,226}$. Historically for example, $\mathrm{n}$-doped $\mathrm{TiO}_{2}$ is able to bleach organic contaminants faster than pure $\mathrm{TiO}_{2}$, but unable to efficiently evolve molecular oxygen under visible irradiation, even in the presence of scavengers ${ }^{42}$. Analogously, both rhombic dodecahedrons and $\mathrm{Ag}_{3} \mathrm{PO}_{4}$ cubes perform much worse for water photooxidation, which is probably due to their high number of unfavourable surface reaction sites. The mixed faceted standard $\mathrm{Ag}_{3} \mathrm{PO}_{4}$ sample presents an intermediate activity, which is expected considering there are a mixture of all exposing facets.

\subsection{Conclusions}

Initially, different sodium based phosphate precursors were used to synthesise three series of $\mathrm{Ag}_{3} \mathrm{PO}_{4}$ crystals all with irregular morphology. Furthermore, synthesis was also carried out in the absence, and presence of water and ethanol as ion exchange solvents. These initial samples (series A, B and C) demonstrated varying abilities to photooxidize water, yet there was no correlation between particle size or BET surface area. Indirectly 
therefore, it was deduced that using large volumes of ethanol yielded a precipitate with a cleaner surface, containing little or no surface species such as sodium or atomic hydrogen. Anhydrous precursors also demonstrated better OERs in comparison to compounds whose precursors contained water. Despite this, it was possible to synthesise a sample (C1) which demonstrates an activity comparable to the study by $\mathrm{Yi}$ et al (in fact, it was better by ca. $20 \%$ ).

In light of collaborators' DFT calculations, it was then decided that controlling the exposing facet of $\mathrm{Ag}_{3} \mathrm{PO}_{4}$ crystals could potentially lead to an understanding over what determines the governing factor of oxygen evolution from water. It was already apparent from series A, B and C that the BET surface area did not influence the OER.

Using method $\mathrm{C}$ as a starting point, tetrahedral $\mathrm{Ag}_{3} \mathrm{PO}_{4}$ crystals composed of $\{111\}$ terminated surfaces were synthesised by using $\mathrm{H}_{3} \mathrm{PO}_{4}$ to control the reaction kinetics of crystal growth. The experimental results show that the synthesised tetrahedrons not only exhibit nearly unity internal quantum yield at $400 \mathrm{~nm}$ but also boast a high quantum yield $(>80 \%)$ over a very wide wavelength range, from the UV to $500 \mathrm{~nm}$. The activity for water photooxidation is nearly 12 times greater than $\{100\}$ and $\{110\}$ faceted crystals which were synthesised using a modified method based on the work of $\mathrm{Bi}$ et al. Tetrahedral $\mathrm{Ag}_{3} \mathrm{PO}_{4}$ crystals also show $240 \%$ and $650 \%$ enhancement compared with benchmark photocatalysts, $\mathrm{Ag}_{3} \mathrm{PO}_{4}$ (mixed facets, sample $\mathrm{C} 1)$ and $\mathrm{BiVO}_{4}$, respectively. In addition, the $\{111\}$ terminated crystals show an underestimated TOF min $^{-1}$ of $2.6 \times 10^{-2}$ and turnover number of ca. 5 , even though the particle size is very large $(>2$ $\mu \mathrm{m})$, and surface area is very small. The excellent performance of $\{111\}$ faceted $\mathrm{Ag}_{3} \mathrm{PO}_{4}$ tetrahedral crystals is attributed a cooperative effect between high mobility of holes and high surface energy.

The hole mass along the [111] direction is much smaller compared to both $\{110\}$ and $\{100\}$ terminated $\mathrm{Ag}_{3} \mathrm{PO}_{4}$ crystals, demonstrating that hole migration without recombination towards the $\{111\}$ facet is statistically more likely to occur. Since water oxidation is the rate determining step in water splitting, and occurs on a slow timescale (ms), the likelihood of electron hole recombination is much larger if the hole mass is larger/hole mobility is smaller. Thus a higher mobility of holes favours a faster and more efficient transport of holes to the surface and water for oxidation reactions. The high surface energy then dramatically enhances the catalytic 
ability at the surface of $\{111\}$ facets, due to more step sites and kinks as shown in the tetrahedral morphology. Whilst not being able to exactly identify the number of kinks or steps, on closer inspection of Figure 29, it is apparent that there is an absence of phosphorus bonds at the $\{111\}$ surface, a result of cleaving P-O bonds when a $\{111\}$ facet is formed. It is also known that the active site for water photooxidation is the oxygen atom - hence there are more oxygen atoms per unit surface area, due to the absence of phosphorus. Therefore the presence of phosphorus at the surface might be detrimental to the photooxidation of water.

In general, the findings provide an effective way to improve the efficiency of a photocatalyst; by controlling the exposing facet. The synthesis preparation method also provides useful information for a reproducible, surfactant free and facile way to control surface facet and bulk properties. With relation to the aim of the thesis, the chapter demonstrates the facet effect for water photooxidation, which dramatically increases OER and internal quantum yield in comparison to non-faceted crystalline photocatalysts.

An opposing argument for the importance of developing a water photooxidation, which is viewed as the key to water splitting due to the temporal difficulties, is the development of a photocatalyst which is able to produce hydrogen from water under visible light. A key problem seen in many photocatalysts is that the positioning of the stable O2p orbital is so deep (located around $+3 \mathrm{eV}$ ), that visible light absorption is limited if the compound also possesses a conduction band negative enough to reduce protons to $\mathrm{H}_{2}$. It is possible to dope the band structure to add sub band gap states and create visible light excitation centres (e.g. Rh or $\mathrm{Cr}$ doped $\mathrm{SrTiO}_{3}$ ), however this approach can never achieve quantum yields high enough required for hydrogen synthesis due to the lack of electronic states present at a acceptor/donor level. Therefore, considering the surveyed literature, the next chapter will thus focus on the feasibility and advancement of a newly discovered, undoped, stable photocatalyst for hydrogen production from water under visible light, graphitic carbon nitride. 


\section{Hydrogen evolving photocatalyst development}

This chapter explores the feasibility of $\mathrm{g}-\mathrm{C}_{3} \mathrm{~N}_{4}$ as a photocatalyst for hydrogen production from water. Graphitic carbon nitride was synthesised via thermal decomposition, using 4 different precursors, at various calcination temperatures, calcination ramp rates, cleaning techniques, and cocatalyst element/weighting. A novel strategy was found to make highly efficient $\mathrm{g}-\mathrm{C}_{3} \mathrm{~N}_{4}$ for $\mathrm{H}_{2}$ production from urea, resulting in an internal quantum yield for hydrogen production of $26.5 \%$ at $400 \mathrm{~nm}$, over an order of magnitude larger than previously reported values ${ }^{227}$. In order to determine the reason for the high reactivity, in comparison to previously documented examples, a series of characterisation methods were employed. TGA-DSC-MS was used to monitor the formation mechanism of $\mathrm{g}-\mathrm{C}_{3} \mathrm{~N}_{4}$ derived from both urea and thiourea - which was found to be drastically different during the primordial stages of synthesis. In the case of urea derived $\mathrm{g}-\mathrm{C}_{3} \mathrm{~N}_{4}$, it is shown that the high surface area and porosity is created by the continuous loss of $\mathrm{CO}_{2}$, a result not seen before. Formaldehyde $\left(\mathrm{CH}_{2} \mathrm{O}\right)$ is also continually produced throughout the decomposition of urea to $\mathrm{g}-\mathrm{C}_{3} \mathrm{~N}_{4}$, a process which is proposed to reduce the percentage of hydrogen/protons in carbon nitride.

Using the N1s core level data from XPS, it is shown that from sample to sample, there is a correlation between surface hydrogen/proton content and hydrogen production rate. A larger surface hydrogen/proton content leads to a lower hydrogen production rate. This trend is further confirmed by bulk hydrogen analysis carried out by elemental analysis. It has been shown in previous studies that a less polymeric carbon nitride intrinsically has more hydrogen/protons due to breakages, but it was unclear as to why this leads to a less effective photocatalyst. XRD analysis confirms that a greater level of condensation, and thus a less polymerised $\mathrm{g}-\mathrm{C}_{3} \mathrm{~N}_{4}$ leads to a less active photocatalyst.

In collaboration with Dr. Stephen Shevlin \& Prof. Z. Xiao Guo, using DFT calculations, we demonstrate that increasing the hydrogen/proton content causes a positive shift in the conduction band edge (with respect to NHE), therefore reducing the driving force for reduction reactions. As shown by TDDFT excess protonation localizes photoelectrons at non-active redox sites, which is further detrimental to the photocatalytic ability. 


\subsection{Introduction}

Very recently, a stable, organic photocatalyst, graphitic carbon nitride (g$\mathrm{C}_{3} \mathrm{~N}_{4}$ ), was found to show sufficient redox power to dissociate water under visible light in a suspension ${ }^{151}$. However the latest documented quantum yields for $\mathrm{H}_{2}$ production from water using g- $\mathrm{C}_{3} \mathrm{~N}_{4}$ do not exceed $4 \%$ (excluding dye-sensitized systems) ${ }^{151,160,161,162,163,164,165,166,167,168}$, which is still unsatisfactory for industrial applications ${ }^{66}$. Graphitic carbon nitride is composed of extremely abundant elements and is non-toxic with proven stability, both thermally and in solutions of $\mathrm{pH} 1$ to 14 . Therefore if an effective and facile strategy is devised to improve its energy conversion efficiency, it can potentially meet all the aforementioned three requirements for a practical photocatalyst.

The structure of carbon nitride has been debated since initial reports in 1834; with melon, melem and melam structures all being proposed ${ }^{152,153,154}$. It is now accepted that the graphitic form of carbon nitride, which is photocatalytically active, is initially composed of stable C-N heptazine (tris-triazine) sheets, hydrogen bonded in a zig-zag formation ${ }^{155}$. Graphitic carbon nitride has a rather unique semiconductor structure; the valence band edge is dominated by nitrogen $p_{z}$ orbitals, and the conduction band edge is dominated by carbon $p_{z}$ orbitals, with a significant contribution from the $p_{z}$ orbitals of the edge nitrogen atoms (twofold-bound). These are all contained within one heptazine unit ${ }^{151}$. The heptazine unit connecting planar nitrogen however does not contribute to the valence band edge. Upon further condensation and removal of $\mathrm{NH}_{3}$ groups, heptazine units continue to polymerise to form what is now conventionally known as graphitic carbon nitride $\left(\mathrm{C}_{\mathrm{x}} \mathrm{N}_{\mathrm{y}} \mathrm{H}_{\mathrm{z}}\right)^{156,157}$. However, as shown both experimentally and theoretically, there are different degrees of polymeric condensation; which can greatly influence the activity ${ }^{156,157,158,159,161}$. From previous findings, it seems that it is not the most condensed graphitic carbon nitride which demonstrates the best activity, but the most polymeric; defects are seemingly detrimental to electron and hole localisation at termination sites ${ }^{151}$. Residual hydrogen, which is present in nearly all carbon nitride structures (as a result of incomplete perfect condensation), appears when this connecting amino $\mathrm{sp}^{2}$ bond is broken, and in principal the amount rises as the polymer begins to buckle. A higher temperature results in in increased condensation; eventually buckling the graphitic structure, and decreasing activity ${ }^{161}$. Up until now however, there has been little direct evidence detailing the activity of $\mathrm{g}-\mathrm{C}_{3} \mathrm{~N}_{4}$ for hydrogen production to the structure and in particular surface features of the compound. 


\subsection{Methodology}

\subsubsection{Photocatalytic analysis}

Reduction reactions (protons to $\mathrm{H}_{2}$ ) were used to analyse the photoactivity of various types of $\mathrm{g}-\mathrm{C}_{3} \mathrm{~N}_{4}$. All reactions were carried out in a custom Pyrex $^{\circledR}$ batch reactor cell $(3.6 \mathrm{~cm}$ diameter of reactor window), which was thoroughly purged with argon prior to radiation. Gas concentration analysis was performed using a GC (Varian 430-GC, TCD, argon carrier gas 99.999\%).

Table 8. Table showing the actual wt\% of some metal precursors.

\begin{tabular}{|c|c|c|c|}
\hline Metal & Precursor & $\begin{array}{c}\text { Value of 'x' }- \\
\text { number of } \\
\text { molecules of } \\
\mathrm{H}_{2} \mathrm{O}\end{array}$ & $\begin{array}{c}\text { Actual wt\% of } \\
\text { metal in precursor } \\
\text { (according to } \\
\text { certificate of } \\
\text { analysis) }\end{array}$ \\
\hline $\begin{array}{c}\text { Platinum, } \\
\text { Pt }\end{array}$ & $\mathrm{H}_{2} \mathrm{PtCl}_{6} \cdot\left(\mathrm{H}_{2} \mathrm{O}\right)_{6}$ & 6 & 37.90 \\
\hline $\begin{array}{c}\text { Gold, Au } \\
\text { Ruthenium, } \\
\text { Ru (III) }\end{array}$ & $\mathrm{HAuCl}_{4} \cdot \mathrm{xH}_{2} \mathrm{O}$ & $3-4$ & 49.96 \\
\hline
\end{tabular}

For a typical reduction reaction, the photocatalyst $(0.02 \mathrm{~g})$ was suspended and subsequently sonicated in a deionised water/hole scavenger mixture (230 $\mathrm{cm}^{3}$ total volume; $210 \mathrm{~cm}^{3}$ DI water, $20 \mathrm{~cm}^{3}$ hole scavenger). A cocatalyst was then deposited onto $\mathrm{g}-\mathrm{C}_{3} \mathrm{~N}_{4}$ using an in situ photodeposition method $^{36}$. Stock solutions of deionised water and precursors (Pt: $\left.\mathrm{H}_{2} \mathrm{PtCl}_{6} \cdot\left(\mathrm{H}_{2} \mathrm{O}\right)_{6}, \quad \mathrm{Au}: \mathrm{HAuCl}_{4} \cdot \mathrm{xH}_{2} \mathrm{O}, \quad \mathrm{Ru}: \mathrm{RuCl}_{3} \cdot \mathrm{xH}_{2} \mathrm{O}\right)$ were made beforehand, and a set volume added according to the required weight of metal (various wt\%, Table 8). The reactor was sealed, purged with Ar gas for 1 hour, and then irradiated for 1 hour under full arc irradiation using a 
$300 \mathrm{~W}$ Xe lamp (TrusTech PLS-SXE 300/300UV). During a one hour period, periodic measurements were taken to determine if hydrogen was being produced at a stable rate, and thus, if photodeposition had occurred correctly. The reactor was then purged a second time, prior to irradiation, limited by a $395 \mathrm{~nm}$ long pass filter (Comar Optics).

Internal quantum yield (IQY, \%) was measured by inserting an appropriate band pass filter $(365,400,420,500 \mathrm{~nm}, \lambda \pm 10 \mathrm{~nm}$ at $10 \%$ of peak height, Comar Optics) in front of a $300 \mathrm{~W}$ Xe light source (TrusTech PLS-SXE $300 / 300 \mathrm{UV})$ to obtain the correct wavelength. The sample preparation procedure is analogous to a hydrogen evolution measurement and the only difference is $0.1 \mathrm{~g}$ photocatalyst is used to ensure complete light absorbance by the photocatalysts. The light intensity was measured at 5 different points to obtain an average intensity, using a photodetector coupled with an optical power meter (Newport Spectra). The intensity of the incoming light was also lowered using a neutral density filter (50\%, Comar Optics),

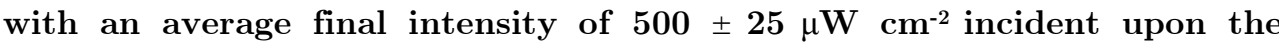
photocatalyst. The efficiency was then calculated using the following formula (ignoring reflected/scattered photons); IQY $(\%)=(2 \times$ amount of $\mathrm{H}_{2}$ molecules evolved / number of photons absorbed) $\times 100$. The turnover number (TON) in terms of cocatalyst was calculated after 6 hours by using the following formula; TON $=$ moles of $\mathrm{H}_{2}$ molecules evolved / moles of active sites (platinum on the photocatalyst), for example:

$$
\begin{gathered}
\text { TON }=\frac{\text { moles of } \mathrm{H}_{2} \text { evolved }}{\text { moles of platinum on photocatalyst }} \\
=\frac{1.972 \mathrm{mmol}}{0.0006 \mathrm{~g}(\mathrm{Pt}) \times\left(\frac{1}{195.084 \mathrm{~g} \mathrm{~mol}^{-1}}\right)} \\
=\frac{1.972 \mathrm{mmol}}{0.003 \mathrm{mmol}} \\
T O N \sim 650
\end{gathered}
$$

\subsubsection{Synthesis techniques}

Graphitic carbon nitride was synthesised using different precursors; urea, dicyandiamide (DCDA), thiourea and cyanamide (Sigma-Aldrich 99.999 $\%)^{158,159}$. In a typical run, the precursor was put in a lidded high quality alumina crucible, then placed inside a muffle furnace and heated at different 
ramp rate (ranging from to 5 to $25{ }^{\circ} \mathrm{C} /$ minute), and finally held at a designated temperature (ranging from 550 to $650{ }^{\circ} \mathrm{C}$ ) for several hours. The resultant powders were then washed with water, $\mathrm{HCl}, \mathrm{NaOH}$ and once again with water to remove all unreacted and potentially detrimental surface species. The products were denoted as $\mathrm{g}-\mathrm{C}_{3} \mathrm{~N}_{4}$ (precursor). The procedure was then carefully optimised to give the highest activity for $\mathbf{H}_{2}$ production from water. Carbon nitride synthesised from melamine and cyanuric acid (MCA) in dimethyl sulfoxide (DMSO), was provided by Kaipei Qiu at UCL Chemistry ${ }^{228}$. Briefly, melamine $(3.96 \mathrm{mmol})$ was dissolved in $20 \mathrm{~cm}^{3}$ DMSO. Equimolar cyanuric acid was dissolved separately in $10 \mathrm{~cm}^{3} \mathrm{DMSO}$. The two solutions were kept separate at $30{ }^{\circ} \mathrm{C}$ and then mixed for 10 minutes. MCA-DMSO crystals were then made after storing on a petri dish for 2 days in fume hood. Afterwards, the crystals were heated to $600{ }^{\circ} \mathrm{C}$ in nitrogen with a ramp rate of $2.3^{\circ} \mathrm{C} / \mathrm{min}$, and after cooling, $\mathrm{g}-\mathrm{C}_{3} \mathrm{~N}_{4}$-MCA-DMSO-30-600 was formed.

\subsection{Results and discussion}

Graphitic carbon nitride was synthesised from urea, DCDA, thiourea under identical conditions in order to determine to what effect the precursor has on the final product. XRD was first used to investigate and verify the crystal structure of the samples. Figure 41 (A), shows there is a very small difference of in terms of peak position $(2 \theta)$ in the XRD patterns between samples. The patterns all exhibit peaks at $13.0^{\circ}(\mathrm{d}=0.681 \mathrm{~nm})$ and $27.4^{\circ}$ $(\mathrm{d}=0.326 \mathrm{~nm})$, corresponding to the approximate dimension of the tri-striazine unit, and the distance between graphitic layers respectively. However on closer inspection, it is evident that the intensity/height of the $27.4^{\circ}$ peak varies between sample, following the order urea < thiourea < DCDA (Figure 41 (B)). This implies that the most crystallised/condensed sample is DCDA, and urea the least, as a more intense peak implies that there is more regular repetitions between graphitic layers. 

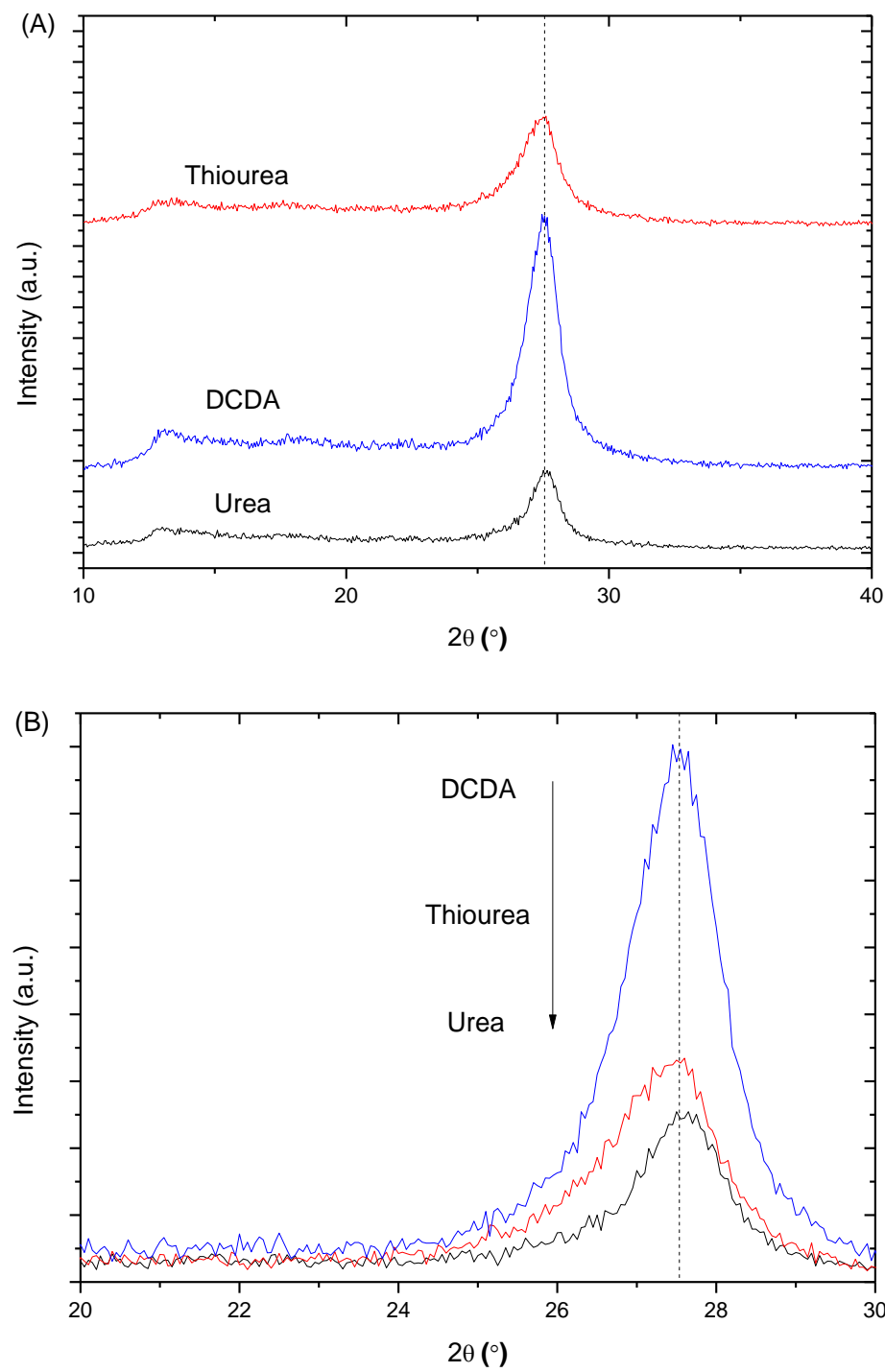

Figure 41. XRD patterns of $\mathrm{g}-\mathrm{C}_{3} \mathrm{~N}_{4}$ synthesised using different precursors using $5{ }^{\circ} \mathrm{C}$ ramp rate, $600{ }^{\circ} \mathrm{C}, 4$ hours hold time.

The ATR-FTIR spectra shown in Figure 42 illustrates the typical bands found in graphitic carbon nitrides, with varying intensity, due to the difference in compressibility between samples - physically the urea sample seems less dense and more compressible in comparison to DCDA and thiourea samples (this is due to a larger surface area, discussed later). As annotated, the peaks between 1000 and $1750 \mathrm{~cm}^{-1}$ are representative of the stretching and bending modes of nitrogen containing heterocycles, and the broad feature at $3250 \mathrm{~cm}^{-1}$ is assigned to stretching modes of -NH- groups. Despite peaks being more distinct in the urea sample, the intensity ratio 
between -NH- and C-N heterocycles descends in the order DCDA > Thiourea > urea; indicating that there is possibly less $-\mathrm{NH}$ - in $\mathrm{g}-\mathrm{C}_{3} \mathrm{~N}_{4}$ prepared using urea.

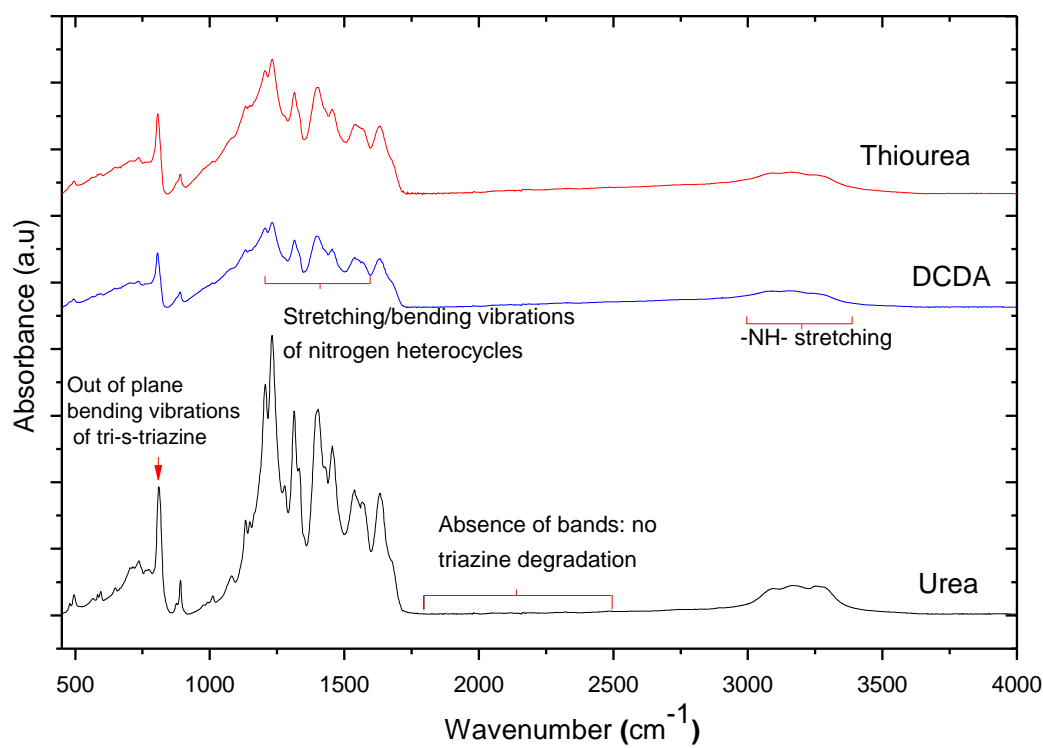

Figure 42. ATR-FTIR spectra of powdered graphitic carbon nitride; annotated are characteristic bands for $\mathrm{g}-\mathrm{C}_{3} \mathrm{~N}_{4}{ }^{229}$.

Figure 43 shows the UV-Vis spectra of the different $g-C_{3} N_{4}$ samples. All three samples exhibit the characteristic absorption of $\mathrm{g}-\mathrm{C}_{3} \mathrm{~N}_{4}$ at around 380 nm which is assigned to $\pi-\pi^{*}$ transitions normally seen in ring systems/heterocyclic aromatics ${ }^{161}$. The shape of the absorption profile is analogous to a semiconductor band gap, with varying approximate band gaps. The sample synthesised from thiourea shows the largest absorption, with an edge around $455 \mathrm{~nm}$. The DCDA derived sample absorbs up to ca. $450 \mathrm{~nm}$, whilst the sample fabricated using urea exhibited a visible blue shift, absorbing light up to approximately $420 \mathrm{~nm}$. All the samples have a small absorption tail, probably due to some sub band gap states from classically forbidden $n-\pi^{*}$ transitions ${ }^{161,230}$. 


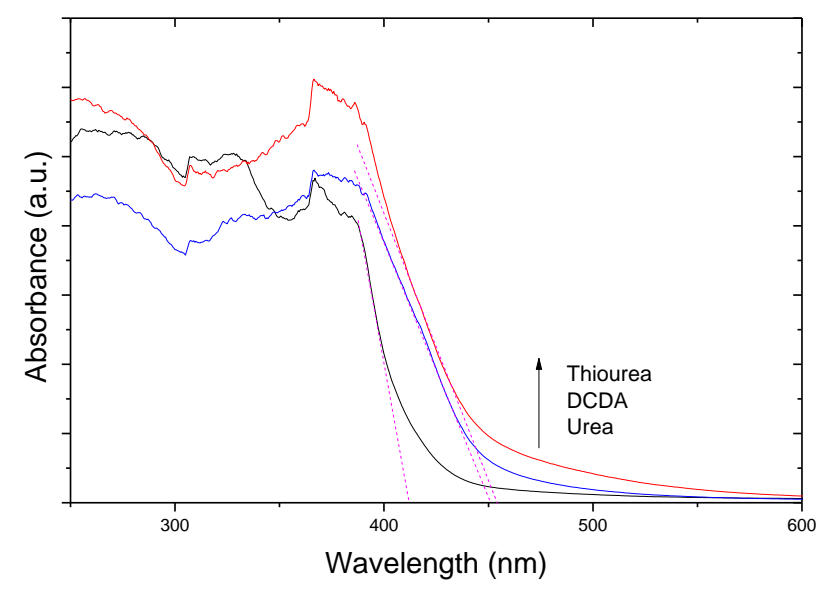

Figure 43. UV-Vis absorbance spectra for graphitic carbon nitride powders synthesized using different precursors at $600{ }^{\circ} \mathrm{C}$.

Raman spectroscopy allows the fine structure of the three samples to be examined in detail, as shown in Figure 44. From $1200-1700 \mathrm{~cm}^{-1}$ a series of peaks dominate, attributed to $\mathrm{C}-\mathrm{N}$ stretching vibrations, specifically ' $\mathrm{G}$ ' and 'D' band profiles of structurally disordered graphitic carbon and other carbon/nitrogen layered compounds ${ }^{231,232}$. The peak at $980 \mathrm{~cm}^{-1}$ can be assigned to the symmetric $\mathrm{N}$-breathing mode of heptazine, whilst the peak at $690 \mathrm{~cm}^{-1}$ corresponds to the in-plane bending vibrations of the heptazine $\mathrm{C}-\mathrm{N}-\mathrm{C}$ linkages ${ }^{233}$. All peaks therefore confirm the local structure of the samples synthesised from various precursors eventually yields the same product; $\mathrm{g}-\mathrm{C}_{3} \mathrm{~N}_{4}$.

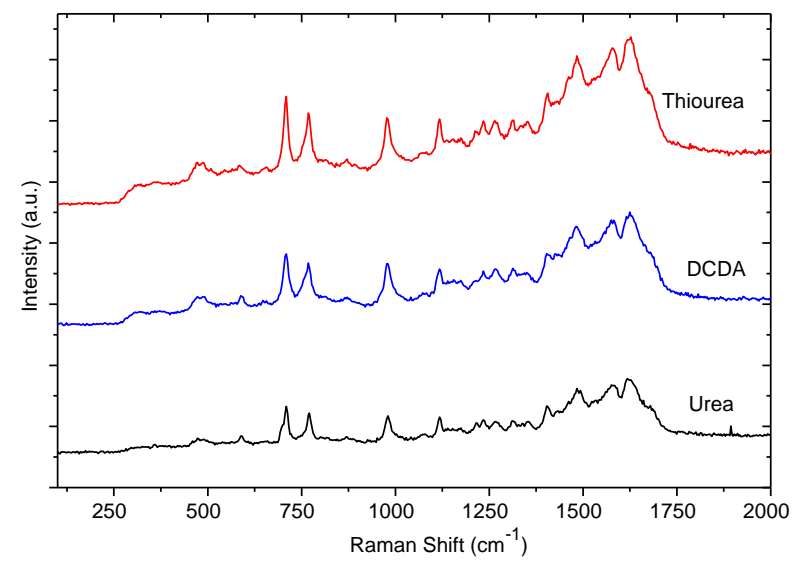

Figure 44. Raman spectra of different carbon nitride samples. 
Transmission electron microscopy (TEM) images in Figure 45 (A) - (C) show the highly amorphous nature of the compounds, and HRTEM micrograph (Figure 45 (D)) indicates pockets of polycrystallinity for the sample prepared using DCDA. Selective Area Electron Diffraction (SAED) in Figure 45 (D) shows diffraction ring patterns, one of which corresponds to $3.2 \AA$, the graphitic layer distance of $\mathrm{g}-\mathrm{C}_{3} \mathrm{~N}_{4}$. Graphitic carbon nitride synthesised from DCDA appears to be the densest structure, whilst the structure of urea-derived $\mathrm{g}-\mathrm{C}_{3} \mathrm{~N}_{4}$ appears more open and porous. This corresponds with the XRD patterns, and also reflects the BET surface areas of the samples (Figure 46). Among the three samples, the urea-derived g$\mathrm{C}_{3} \mathrm{~N}_{4}$ is the least dense, exhibiting the largest surface area and also greatest average pore volume, followed by thiourea-derived $\mathrm{g}-\mathrm{C}_{3} \mathrm{~N}_{4}$, and then DCDA-derived $\mathrm{g}_{-} \mathrm{C}_{3} \mathrm{~N}_{4}$. All isotherms can be classified as type $\mathrm{V}$, demonstrating significant hysteresis and a plateau at $\mathrm{P} / \mathrm{P}_{0}<0.3$.
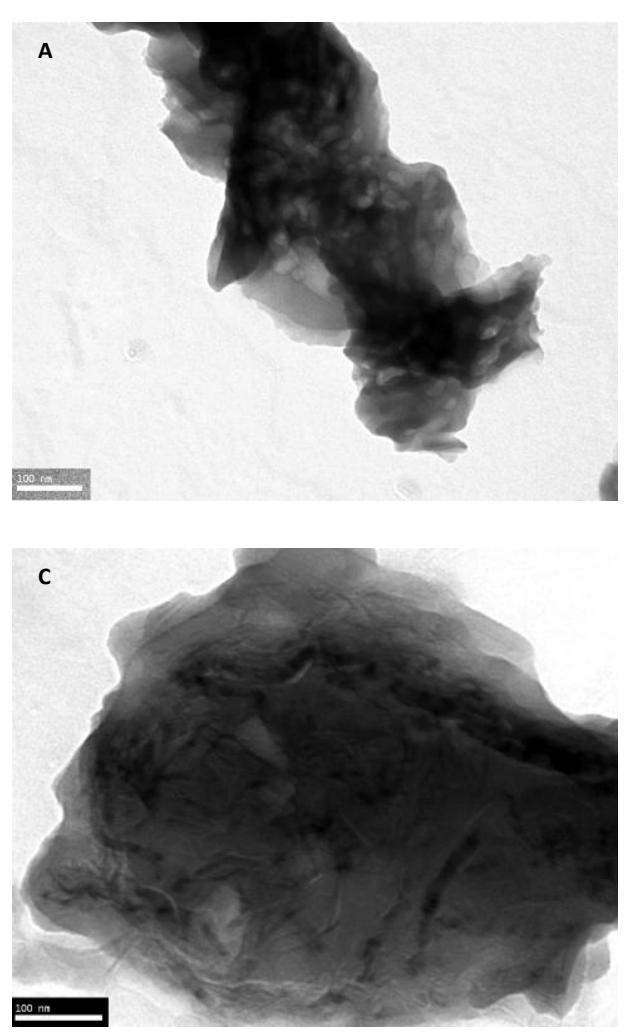
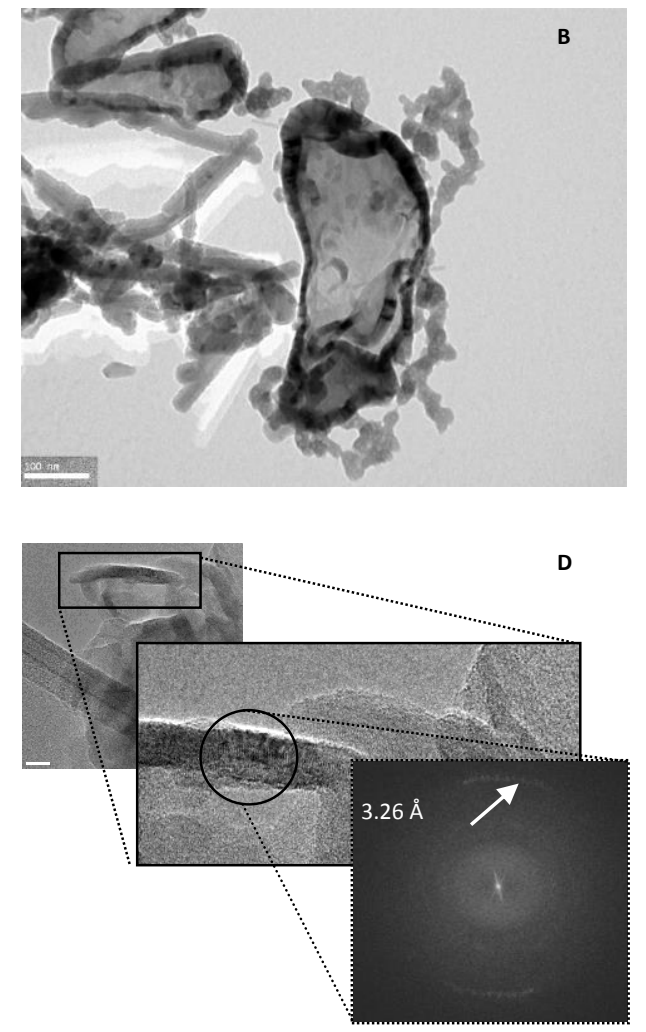

Figure 45. TEM micrographs of $\mathrm{g}-\mathrm{C}_{3} \mathrm{~N}_{4}$ synthesised from: (A) Urea, (B) Thiourea, and (C) DCDA. The micrograph scheme at (D) shows a continual magnification of the $\mathrm{g}-\mathrm{C}_{3} \mathrm{~N}_{4}$ (urea) structure, and the resulting diffraction rings from microcrystalline regions. 


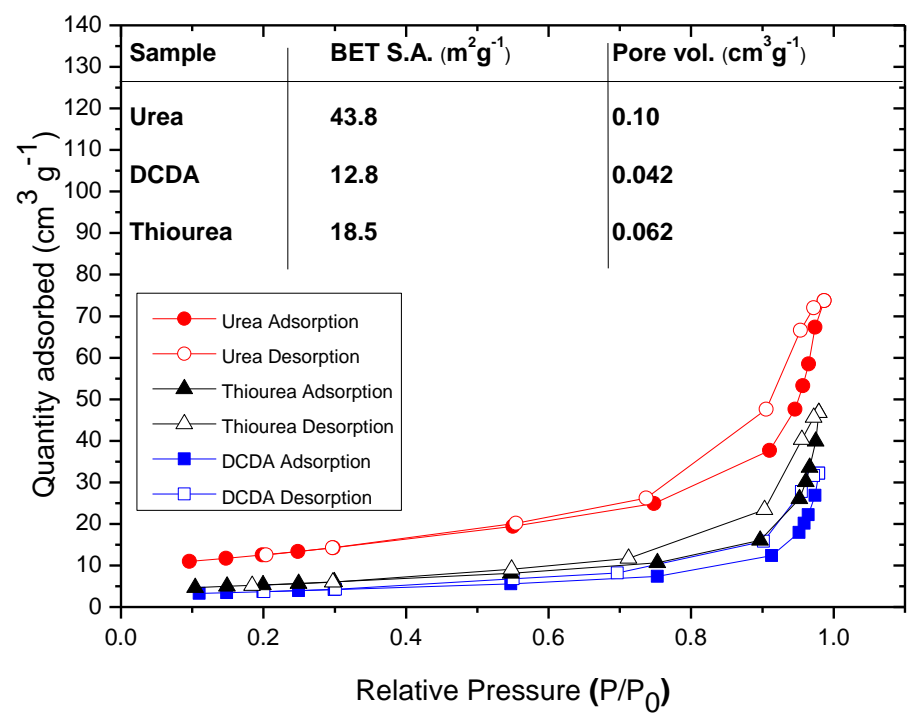

Figure 46. BET nitrogen adsorption/desorption isotherms. All samples were pre-prepared by calcination at $600{ }^{\circ} \mathrm{C}$, for 4 hours, with a ramp rate of $5{ }^{\circ} \mathrm{C} /$ minute.

In order to understand the nature of the low density sample prepared from urea, thermal gravimetric analysis - differential scanning calorimetry mass spectroscopy (TGA-DSC-MS) was undertaken to analyse the sample synthesis route, and the $\mathrm{g}-\mathrm{C}_{3} \mathrm{~N}_{4}$ prepared from thiourea was also analysed for comparison. Interestingly, new phenomena were observed; the origin of the porosity in $\mathrm{g}-\mathrm{C}_{3} \mathrm{~N}_{4}$ synthesised from urea and thiourea (Figure 47 and Figure 48).

According to a previous report, the condensation and polymerisation of cyanamide and dicyandiamide precursors occur with the gradual loss of $\mathrm{NH}_{3}$, while thiourea could go through additional steps of losing not only $\mathrm{NH}_{3}$, but potentially also $\mathrm{CS}_{2}{ }^{158}$. The TGA-MS results confirm this hypothesis, with both $\mathrm{CS}_{2}$ and $\mathrm{CH}_{4} \mathrm{~N}_{2}$ being detected by MS, in addition to $\mathrm{NH}_{3}$ and thiocyanic acid. This accounts for the slight difference in surface area between DCDA and thiourea; both $\mathrm{CS}_{2}$ and $\mathrm{CH}_{4} \mathrm{~N}_{2}$ being trapped in the structure beyond their boiling points and eventually ceasing to evolve at $192{ }^{\circ} \mathrm{C}$ and $310{ }^{\circ} \mathrm{C}$ as recorded by MS. Only trace amounts of $\mathrm{CS}_{2}$ and $\mathrm{CH}_{4} \mathrm{~N}_{2}$ remain at the point of polymerisation $\left(500{ }^{\circ} \mathrm{C}\right)$. Crucially, urea is different from other precursors because of the presence of oxygen; $\mathrm{CO}_{2}$ is initially detected at $150{ }^{\circ} \mathrm{C}$, and continues to evolve slowly along with $\mathrm{NH}_{3}$, which is in a relatively high amount throughout the analysis. Since the 
polymerisation of heptazine to melem happens around $389{ }^{\circ} \mathrm{C}{ }^{151}, \mathrm{CO}_{2}$ can thus aid the formation of pores; graphitic layers will grow around the trapped gas, as shown in the fabrication of high porosity activated carbon ${ }^{234}$. In contrast to previous reports which propose other mechanisms behind the large surface area of urea based carbon nitrides ${ }^{235}$, it is proven here that $\mathrm{CO}_{2}$ is crucial for the formation of the largest pores and the highest surface area.
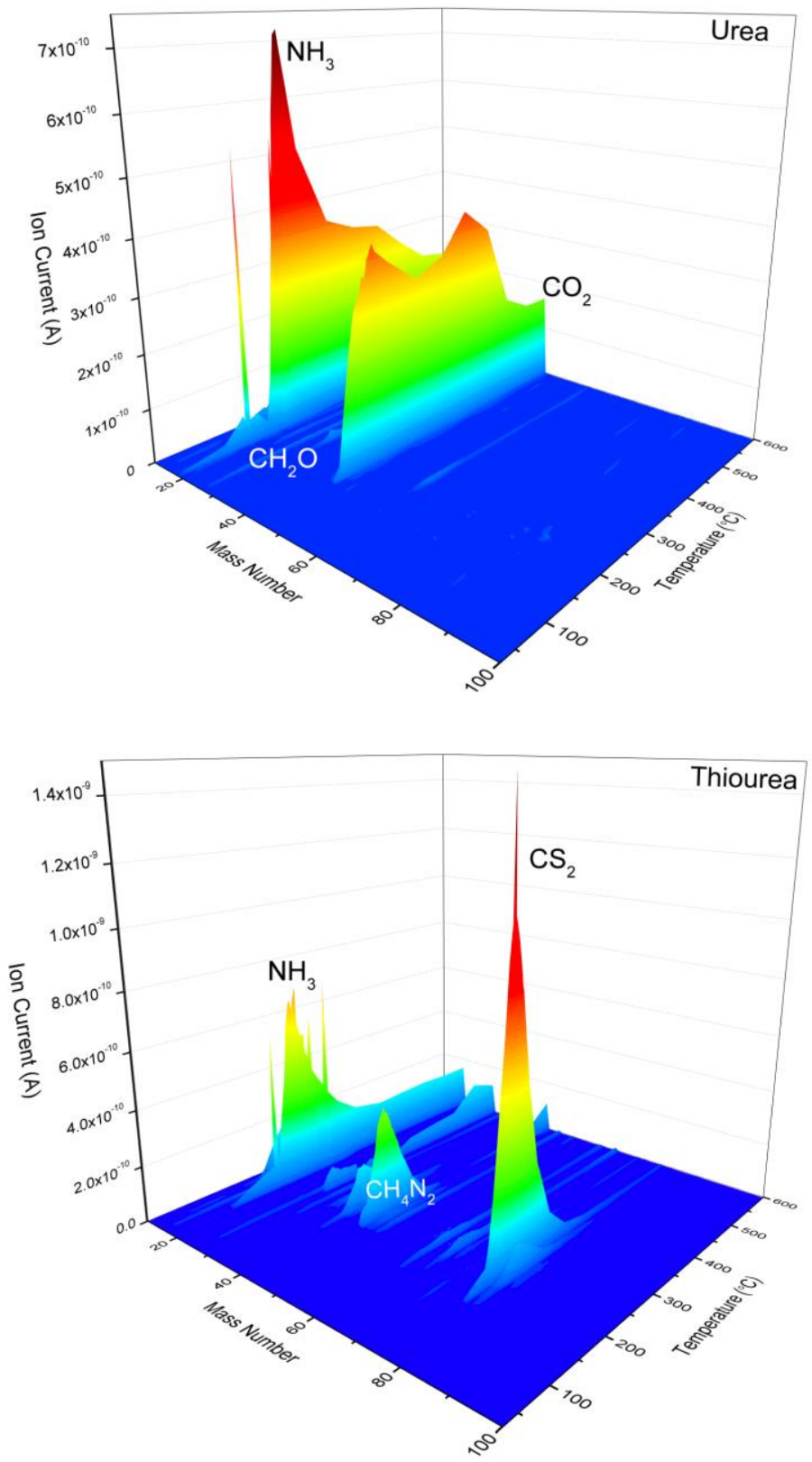
Figure 47. TGA-DSC-MS data from $\mathrm{g}-\mathrm{C}_{3} \mathrm{~N}_{4}$ synthesised from urea and thiourea.

Formaldehyde $\left(\mathrm{CH}_{2} \mathrm{O}\right)$ was detected with a peak starting at $230{ }^{\circ} \mathrm{C}$ and continuing throughout the heating process, possibly as a result of hydrogenation of $\mathrm{CO}$ or $\mathrm{CO}_{2}$. This would suggest that hydrogen is continuously removed from nitrogen in urea when reacting with $\mathrm{CO}$ to form $\mathrm{CH}_{2} \mathrm{O}$, which is then detected by the MS; thereby enabling free nitrogen to covalently bond, forming melamine from precursory intermediate carbon, pinning the electron lone pair to nitrogen and leading to a high degree of polymerisation. This does not take place in the synthesis of $\mathrm{C}_{3} \mathrm{~N}_{4}$ using DCDA/thiourea and is in agreement with the following discussion about the differences in surface chemistry between the three samples.
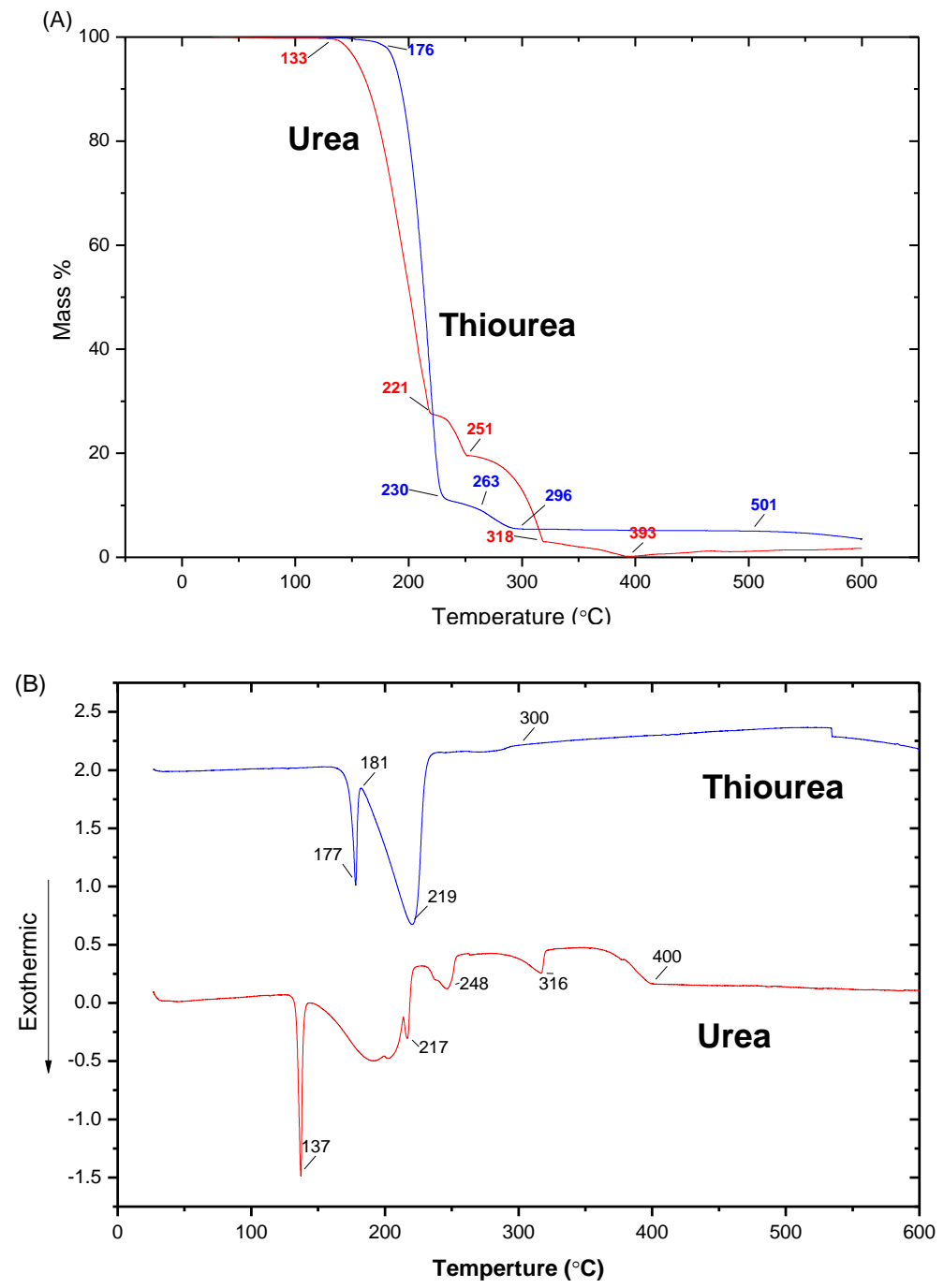
Figure 48. TGA (A) and DSC (B) analysis of urea and thiourea.

Graphitic carbon nitride, synthesised from different precursors $\left(600{ }^{\circ} \mathrm{C}, 5\right.$ ${ }^{\circ} \mathrm{C}$ per minute ramp rate), was tested for hydrogen evolution in an aqueous sacrificial solution containing triethanolamine (TEOA) at room temperature and atmospheric pressure, akin to other reports ${ }^{151,165}$. The fully optimised results are shown in Figure 49 and further summarised in Table 9. As shown in Figure 49 (a) and (b), the urea-derived $\mathrm{g}-\mathrm{C}_{3} \mathrm{~N}_{4}$ exhibits superior hydrogen evolution in comparison to either the widely used DCDA or thiourea derived g- $\mathrm{C}_{3} \mathrm{~N}_{4}$ under both full arc and visible light irradiation. The urea-derived g- $\mathrm{C}_{3} \mathrm{~N}_{4}$ evolves hydrogen at ca. $20,000 \mu \mathrm{mol} \mathrm{hr} \mathrm{hg}^{-1} \mathrm{~g}^{-1}, 15$ times faster than the DCDA derived and 8 times higher than thioureaderived $\mathrm{g}_{-} \mathrm{C}_{3} \mathrm{~N}_{4}$. This is reflected in the TON (over platinum cocatalyst); urea-derived $\mathrm{g}-\mathrm{C}_{3} \mathrm{~N}_{4}$ has a TON of 641.1, much higher than other samples.
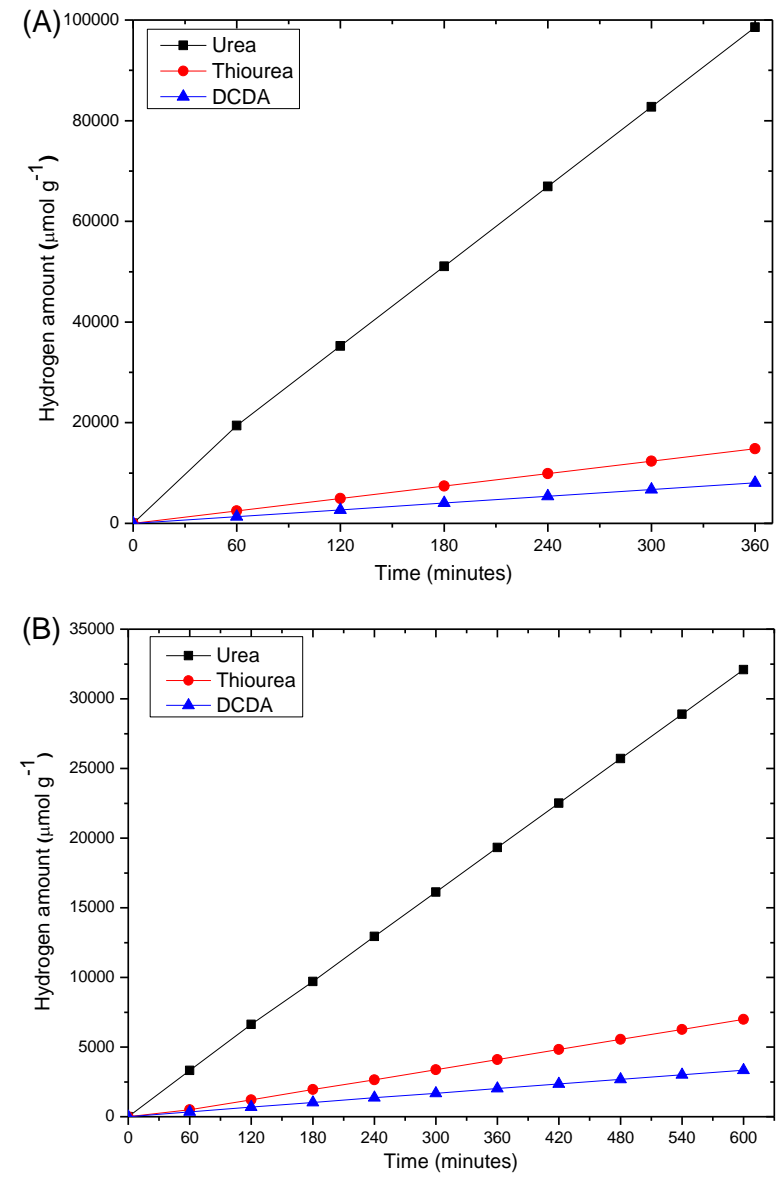

Figure 49. Hydrogen evolution using a $300 \mathrm{~W}$ Xe lamp, $3 \mathrm{wt} \% \mathrm{Pt}$, TEOA as a hole scavenger; (A) Full arc, (B) $\lambda \geq 395 \mathrm{~nm}$. 
Even under visible light irradiation $(\lambda \geq 395 \mathrm{~nm}$, Figure $49(\mathrm{~b}))$, the ureaderived g- $\mathrm{C}_{3} \mathrm{~N}_{4}$ evolves $\mathrm{H}_{2}$ at $3,300 \mu \mathrm{mol} \mathrm{hr} \mathrm{h}^{-1} \mathrm{~g}^{-1}$, nearly 10 times faster than the DCDA-derived $\mathrm{g}-\mathrm{C}_{3} \mathrm{~N}_{4}$ at $300 \mu \mathrm{mol} \mathrm{hr} \mathrm{hr}^{-1} \mathrm{~g}^{-1}$ and nearly 7 times greater than thiourea-derived $\mathrm{g}_{-} \mathrm{C}_{3} \mathrm{~N}_{4}$ at $500 \mu \mathrm{mol} \mathrm{hr} \mathrm{hr}^{-1} \mathrm{~g}^{-1}$ One may conclude that the activity difference is due to surface area; however the urea-derived g$\mathrm{C}_{3} \mathrm{~N}_{4}$ only has a specific surface area (SSA) 3.4 times greater than DCDA derived g- $\mathrm{C}_{3} \mathrm{~N}_{4}$ and 2.4 times of the sample thiourea derived $\mathrm{g}-\mathrm{C}_{3} \mathrm{~N}_{4}$ (Figure 46). Meanwhile, the activity is over 15 times and more than 8 times higher compared to the samples synthesised using DCDA and thiourea respectively. Interestingly, urea derived $\mathrm{g}-\mathrm{C}_{3} \mathrm{~N}_{4}$ calcined at $550{ }^{\circ} \mathrm{C}$ shows a surface area of $83.5 \mathrm{~m}^{2} \mathrm{~g}^{-1}$, double that of the sample calcined at $600^{\circ} \mathrm{C}$. Yet, the activity of the former is only a third of the latter (Figure 50). Jun et al prepared g- $\mathrm{C}_{3} \mathrm{~N}_{4}$ at $550{ }^{\circ} \mathrm{C}(3 \mathrm{~h})$ using urea as a precursor, and reported a hydrogen evolution rate (HER) of $625 \mu \mathrm{mol} \mathrm{h} \mathrm{h}^{-1} \mathrm{~g}^{-1}$, which is similar to the sample prepared here, and thus used as a control ${ }^{228}$. The difference in activity is due to the shorter calcination time employed during sample preparation, which will affect the level of polymerisation of the compound, as shown by Zhang et $\mathrm{al}^{236}$. Curiously, despite being able to absorb less light, the urea derived carbon nitride still outperforms both DCDA and thiourea derived counterparts (Table 9). Therefore, the activity cannot be directly attributed to either surface area or optical absorption.

Table 9. Summary of $\mathrm{g}_{-} \mathrm{C}_{3} \mathrm{~N}_{4}$ properties synthesised from different precursors. $\mathrm{H}_{2}$ evolution rate is using a $300 \mathrm{~W}$ Xe lamp, $3 \mathrm{wt} \% \mathrm{Pt}$, TEOA as a hole scavenger. All samples where synthesised at $600{ }^{\circ} \mathrm{C}$ in air. TON is calculated with respect to Pt catalyst.

\begin{tabular}{|l|l|l|l|l|}
\hline \multicolumn{1}{|c|}{ Sample } & \multicolumn{1}{|c|}{$\begin{array}{c}\text { HER rate } \\
\left(\mu \mathrm{mol} \mathrm{h} \mathbf{~ h}^{-1} \mathbf{g}^{-1}\right)\end{array}$} & $\begin{array}{c}\text { TON } \\
(6 \text { hours })\end{array}$ & $\begin{array}{c}\text { Band edge } \\
(\mathbf{n m})\end{array}$ & SSA $\left(\mathbf{m}^{2} \mathbf{g}^{-1}\right)$ \\
\hline Urea & 19,412 & 641.1 & 415 & 43.8 \\
\hline DCDA & 1,350 & 52.5 & 451 & 12.8 \\
\hline Thiourea & 2,470 & 96.4 & 453 & 18.5 \\
\hline
\end{tabular}


It was found that $600{ }^{\circ} \mathrm{C}$ is the optimal calcination temperature for $\mathrm{g}_{-} \mathrm{C}_{3} \mathrm{~N}_{4}$ when considering the correlation with hydrogen production (Figure 49(a)). This can be explained because of the behaviour of graphitic carbon nitride with temperature. As seen in the TGA-DSC-MS analysis (Figure 47 and Figure 48), the urea-derived g- $\mathrm{C}_{3} \mathrm{~N}_{4}$ continually undergoes loss of $\mathrm{NH}_{3}$ and eventually begins to form heptazine units at $500{ }^{\circ} \mathrm{C}$. However, at this temperature, some of the precursors still remain and full conversion to g$\mathrm{C}_{3} \mathrm{~N}_{4}$ has not been completed. As the temperature rises, polymerisation progresses, and the compound becomes more polymeric in nature; this is reflected in the activity, as it increases with temperature. However, $600{ }^{\circ} \mathrm{C}$ is a critical point; if the temperature reaches $650{ }^{\circ} \mathrm{C}$, then the graphitic structure begins to break down, as full condensation of the network proceeds. As this happens the structure will buckle, and thus the activity will dramatically drop; the electronic structure is distorted and the compound ceases to be an effective photocatalyst, as seen elsewhere ${ }^{161}$. Nonpolymeric carbon nitride, i.e. a melem unit, is not a photocatalyst, whilst g- $\mathrm{C}_{3} \mathrm{~N}_{4}$ is. Therefore it can be hypothesised that a less polymeric $\mathrm{g}-\mathrm{C}_{3} \mathrm{~N}_{4}$, i.e. more breakages in the structure, essentially becomes less photocatalytic, and more inert.

As discussed later, the degree of polymerisation of $\mathrm{g}-\mathrm{C}_{3} \mathrm{~N}_{4}$ affects the protonation status, and thus the compound's ability to function as an effective photocatalyst. The ramp rate of the reaction is also important; Figure 50(b) illustrates that a ramp rate of $5{ }^{\circ} \mathrm{C}$ is clearly superior to 10 and $25{ }^{\circ} \mathrm{C}$, which appears to be a crucial step in enhancing photoactivity. From our experimentation, if the ramp rate is too fast, $\mathrm{g}-\mathrm{C}_{3} \mathrm{~N}_{4}$ has a tendency to sublimate, notably decreasing product yield. Subsequently, since condensation in this case is through the loss of ammonia groups, logic would dictate that sufficient time is necessary, at each polymerisation step, for $\mathrm{NH}_{\mathrm{x}}$ and $\mathrm{CH}_{2} \mathrm{O}$ to be released. If the reaction proceeds slowly, the network has the necessary time to form and proceed through each step, and gradually lose protons from within the structure, and polymerise into an effective photocatalyst.

Different cocatalysts were also deposited onto the surface of urea-derived g- $\mathrm{C}_{3} \mathrm{~N}_{4}$; platinum was found to be the most effective at reducing protons to hydrogen because of the very small overpotential it provides (Figure 50(c)). The weighting was also key; $3 \%$ was found to be the optimum loading, with both $2 \%$ and $4 \%$; providing insufficiently and overly populated active sites, respectively, as reported on other photocatalysts loaded with noble metals 
(Figure 50(d) $)^{131}$. Large platinum loadings could also prevent light adsorption and halt redox reactions. It can also be argued that the weighting could influence size of the platinum particles deposited on the surface, which could then influence the catalytic activity of the system. However, there are no definitive/reliable studies to date, yet this could be a considerable future project if it was possible to produce platinum nanoparticles with very small size distributions.
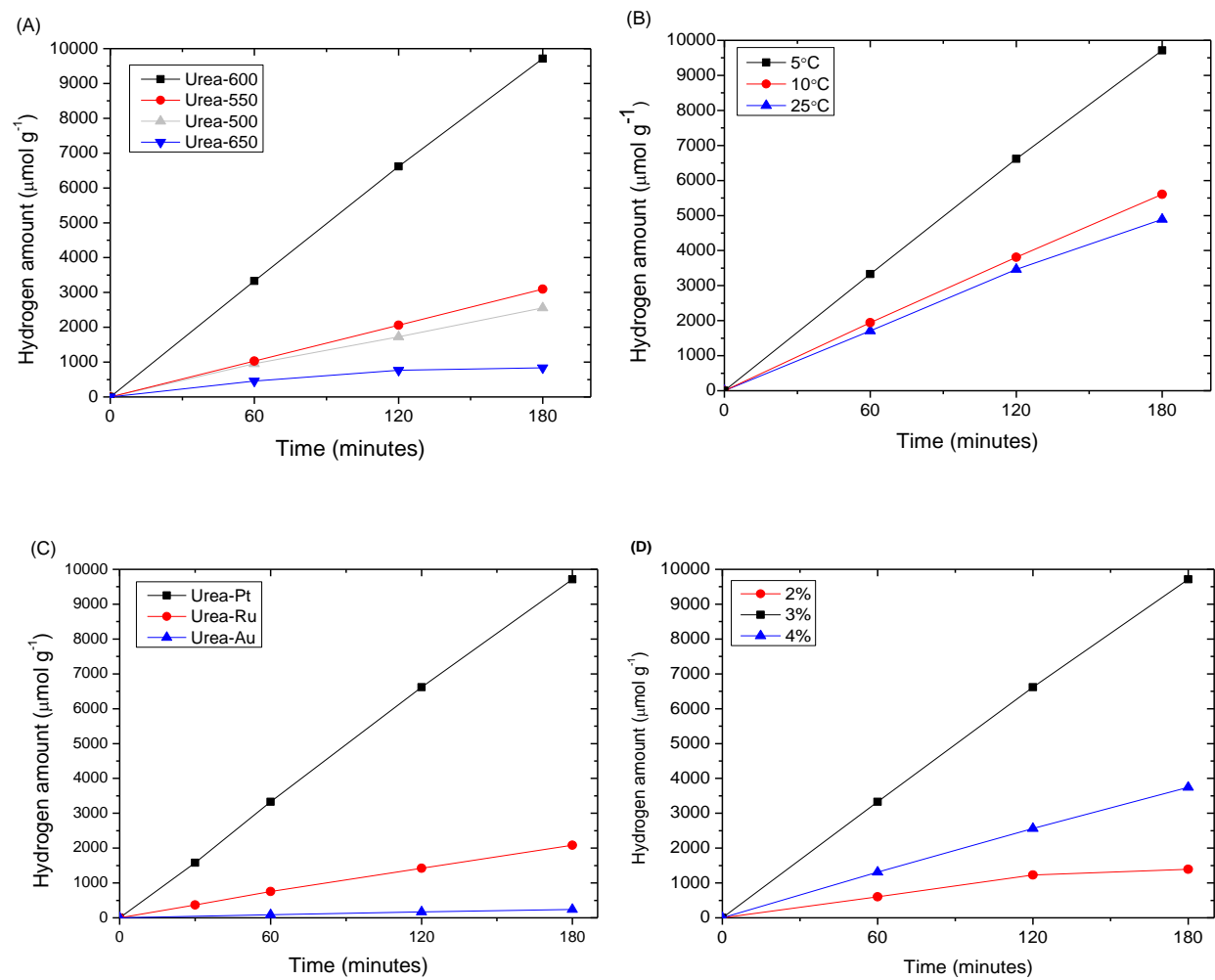

Figure 50. Hydrogen production with various synthesis parameters of urea derived $\mathrm{g}_{-} \mathrm{C}_{3} \mathrm{~N}_{4}(\lambda \geq 395 \mathrm{~nm}$, TEOA hole scavenger, $300 \mathrm{~W}$ Xe lamp). (A) Urea based $\mathrm{g}-\mathrm{C}_{3} \mathrm{~N}_{4}$ synthesized at different temperatures, $4 \mathrm{~h}$ hold time $\left({ }^{\circ} \mathrm{C}\right)$. (B) Urea synthesized at $600{ }^{\circ} \mathrm{C}$ using different ramping temperatures $\left({ }^{\circ} \mathrm{C} /\right.$ minute). (C) $\mathrm{g}-\mathrm{C}_{3} \mathrm{~N}_{4}$ (urea) with different cocatalysts, $3 \mathrm{wt} \%$. (D) Weight percent of $\mathrm{Pt}$ in comparison to photocatalyst (urea, $600{ }^{\circ} \mathrm{C}$ ).

X-ray photoelectron spectroscopy (XPS) studies were undertaken to accurately determine the specific bonding and structure of the samples. In all samples the typical C1s and N1s peaks are observed, similar to other reports ${ }^{235,237}$. A residual O1s peak is also noted, which is likely due to 
calcination in air. XPS spectra from the three samples (urea, thiourea, DCDA) are shown in Figure 51 (N1s XPS) and Figure 52 (C1s and O1s XPS spectra). The O1s level (Figure 52) is composed of two convoluted peaks at 532 and $534 \mathrm{eV}$, respectively, which are assigned to N-C-O (oxygen in the bulk) and adsorbed oxygen ${ }^{238}$, presumably due to air contamination from calcination.

The C1s spectra show bonding of C-C, C-N-C and a trace C-O located at $285.1,288.2$, and $289.2 \mathrm{eV}$, respectively. The spectra of N1s can be fitted to elucidate 4 separate signals, and provides a better idea of the bonding structure, since carbon spectra are susceptible to contamination. The N1s core levels at $398.7,399.7$ and $400.9 \mathrm{eV}$ correspond to $\mathrm{sp}^{2} \mathrm{C}-\mathrm{N}-\mathrm{C}, \mathrm{sp}^{3} \mathrm{H}-\mathrm{N}$ $[\mathrm{C}]_{3}$ and $\mathrm{C}-\mathrm{NH}_{\mathrm{x}}$ (amino functional groups) respectively ${ }^{238}$. The weak peak at $404.4 \mathrm{eV}$ can be attributed to terminal nitrate groups, charging effects, or $\pi$ excitations ${ }^{158,237}$. Hybridized $\mathbf{s p}^{3}$ nitrogen not only has three chemical bonds to carbon, but because of hybridization, can also possess bonded hydrogen perpendicular to the direction of the graphitic layer.

The atomic ratio of various nitrogen bonding configurations in all the three samples were calculated based on XPS area mapping $(3 \times 3)$, using a pointto-point distance of $0.7 \mathrm{~mm}$, in order to give an average, homogenized data set. Figure 53 and Table 10 shows the distinctive trend in bonding ratios versus activity between samples. The ratio of $\mathrm{sp}^{2} \mathrm{C}-\mathrm{N}-\mathrm{C}$ to the sum of $\mathrm{sp}^{3}$ $\mathrm{H}-\mathrm{N}-[\mathrm{C}]_{3}$ and $\mathrm{C}-\mathrm{NH}_{\mathrm{x}}$ bonds (where the latter represents the total amount of protons) is 2.83 in urea, 2.7 for thiourea and only 2.31 in DCDA. As it is part of the heptazine ring, linked by a double and a single bond to two opposing carbons, the $\mathrm{sp}^{2}$ bonded nitrogen is the principle participant that contributes to band gap absorption and therefore is an incredibly important part of the structure. Both hybridized $\mathrm{sp}^{3}$ nitrogen and surface functional amino groups $\left(\mathrm{C}-\mathrm{NH}_{\mathrm{x}}\right)$ are also key features when considering bulk and surface properties. Along with $\mathrm{C}-\mathrm{NH}_{\mathrm{x}}$ bonding, graphitic carbon nitride possesses a positively charged, slightly acidic surface, confirmed by zeta potential measurements (Figure 54). Elemental analysis (EA) further confirms the trend shown in Figure 53; as bulk $\mathrm{H}$ (at\%) increases, the HER

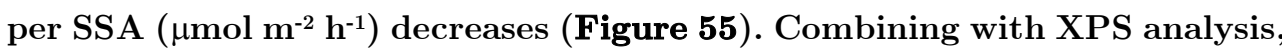
one can conclude that a lower proton concentration leads to a larger HER. 

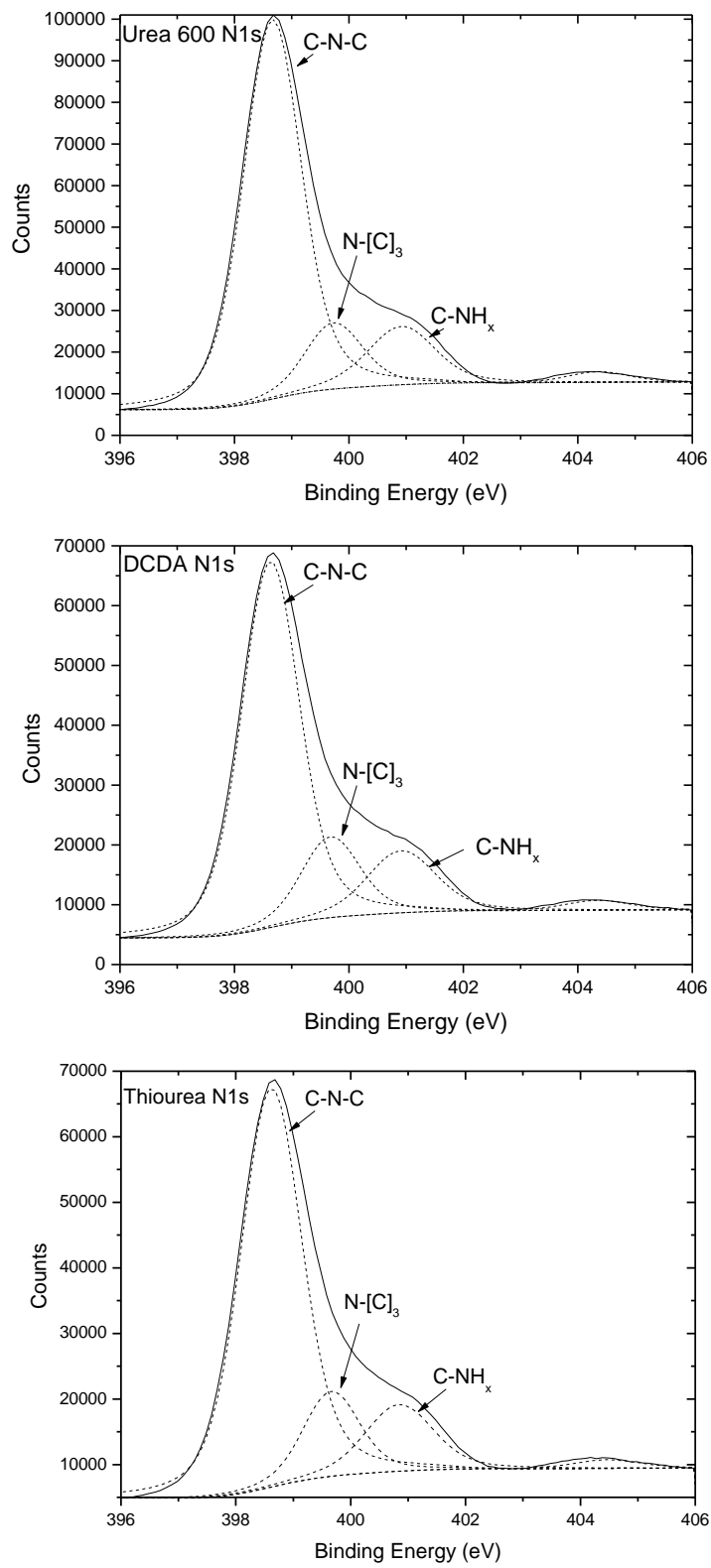

Figure 51. XPS spectra from $\mathrm{g}_{-} \mathrm{C}_{3} \mathrm{~N}_{4}$, for urea, DCDA and thiourea derived samples. 

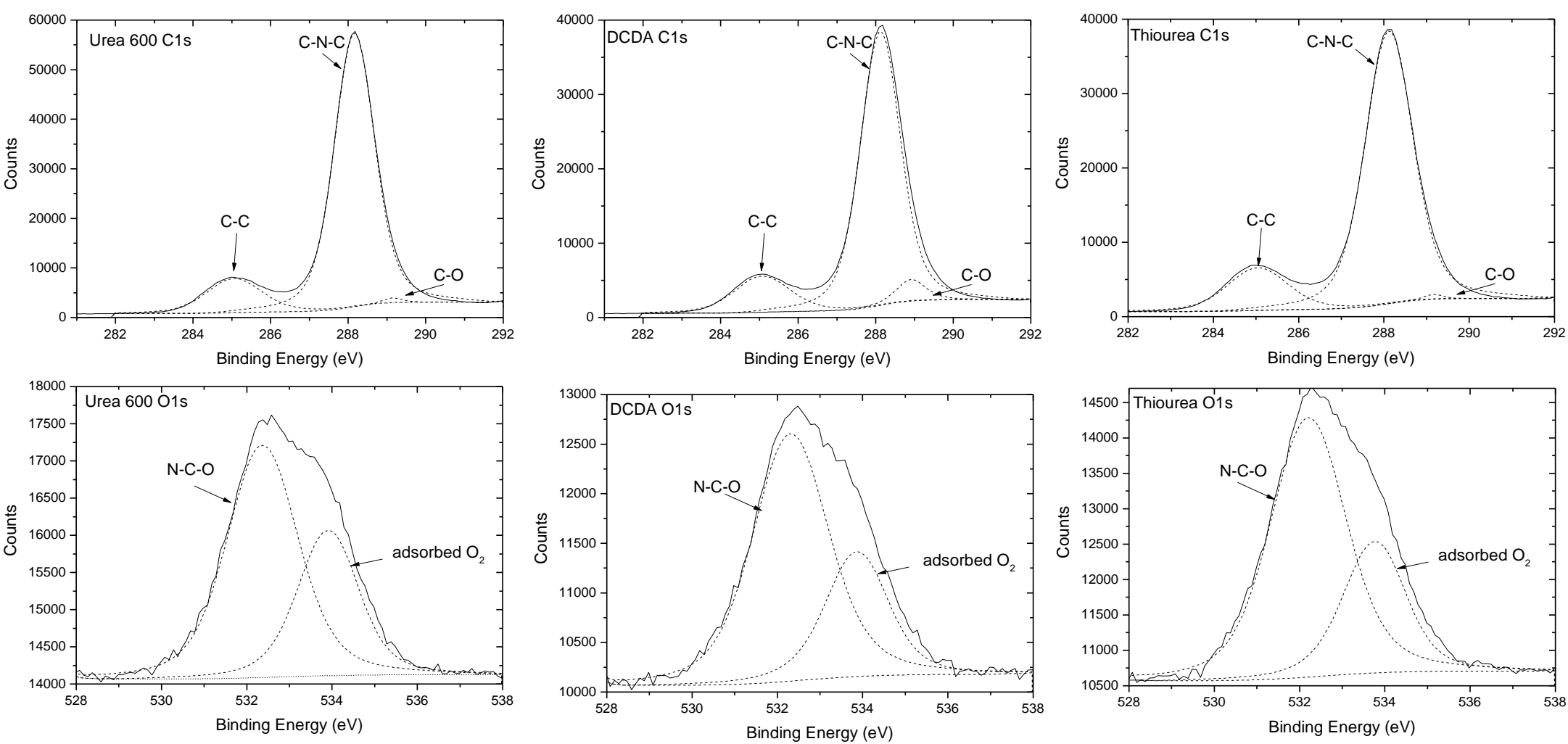

Figure 52. C1s and O1s XPS spectra of $\mathrm{g}-\mathrm{C}_{3} \mathrm{~N}_{4}$ synthesised from different precursors. 


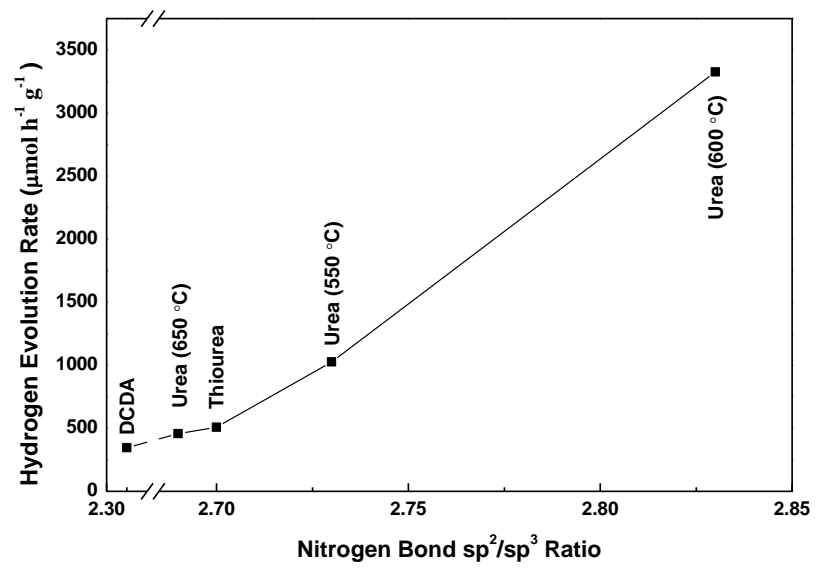

Figure 53. Ratios of bonds within the N1s core level peak in different samples and their comparisons to the HER under visible light $(\lambda \geq 395 \mathrm{~nm})$, indicating decreasing proton concentration dramatically increases photocatalytic activity.

Table 10. Ratios of bonds within the N1s core level peak in different samples and their comparisons to the hydrogen evolution activity.

\begin{tabular}{|c|c|c|}
\hline \multirow[b]{2}{*}{ Sample } & \multicolumn{2}{|r|}{ Ratio } \\
\hline & $\begin{array}{l}\mathrm{sp}^{2} \text { to sum of } \\
\mathrm{sp}^{3} \text { and } \mathrm{C}-\mathrm{NH}\end{array}$ & $\begin{array}{l}\text { Hydrogen evolution activity under } \\
\text { visible light (umol } h^{-1} \mathbf{g}^{-1} \text { ) }\end{array}$ \\
\hline Urea $\left(600{ }^{\circ} \mathbf{C}\right)$ & 2.83 & 3327 \\
\hline Urea $\left(550^{\circ} \mathbf{C}\right)$ & 2.73 & 1027 \\
\hline Thiourea & 2.70 & 508 \\
\hline Urea $\left(650^{\circ} \mathbf{C}\right)$ & 2.69 & 457 \\
\hline DCDA & 2.31 & 345 \\
\hline
\end{tabular}

Table 11. Percentage breakdown of different bonds within the N1s spectrum. 


\begin{tabular}{|c|c|c|c|c|}
\hline & N1s & \multicolumn{3}{|c|}{ Atomic \% of bond (sample calcined at } \\
\hline Bond & $\begin{array}{c}\text { Binding energy } \\
(\mathbf{e V})\end{array}$ & Urea & DCDA & Thiourea \\
\hline $\mathbf{C}-\mathrm{N}-\mathrm{C}\left(\mathbf{s p}^{2}\right)$ & 398.7 & 72.3 & 68.4 & 71.8 \\
\hline $\mathrm{N}-[\mathrm{C}]_{3}\left(\mathbf{s p}^{3}\right)$ & 399.7 & 12.6 & 15.6 & 13.6 \\
\hline $\mathbf{C}-N_{\mathbf{x}}$ & 400.9 & 13.0 & 14.0 & 13.0 \\
\hline $\mathrm{NO}_{2}$ terminal & 404.4 & 2.1 & 2.1 & 1.7 \\
\hline
\end{tabular}

Zeta potential measurements of the three samples show a similar trend to XPS at the operating $\mathrm{pH}(\mathbf{9 . 7 5})$; the urea derived sample has the smallest zeta potential (i.e. smallest level of surface protonation), followed by the thiourea-derived and ultimately the DCDA-derived sample. Thus there is an evident correlation herein between activity and surface protonation; the lower the positive charge on the surface, the higher the activity. The negative charge seen at the slipping plane clearly shows the actual surface is positive; thus in a solution, attracts $\mathrm{OH}^{-}$groups.

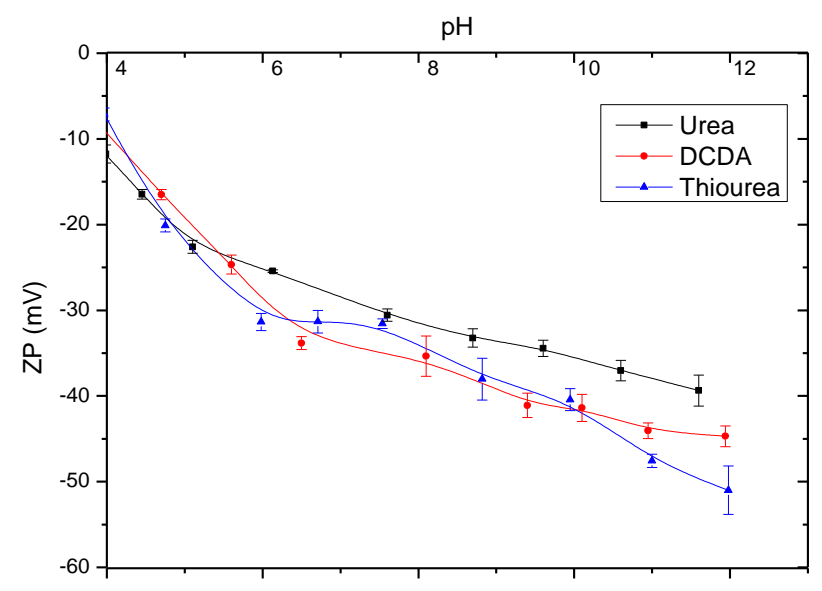

Figure 54. Zeta potential of $\mathrm{g}-\mathrm{C}_{3} \mathrm{~N}_{4}$ synthesised from different precursors $\left(600{ }^{\circ} \mathrm{C}\right)$. 


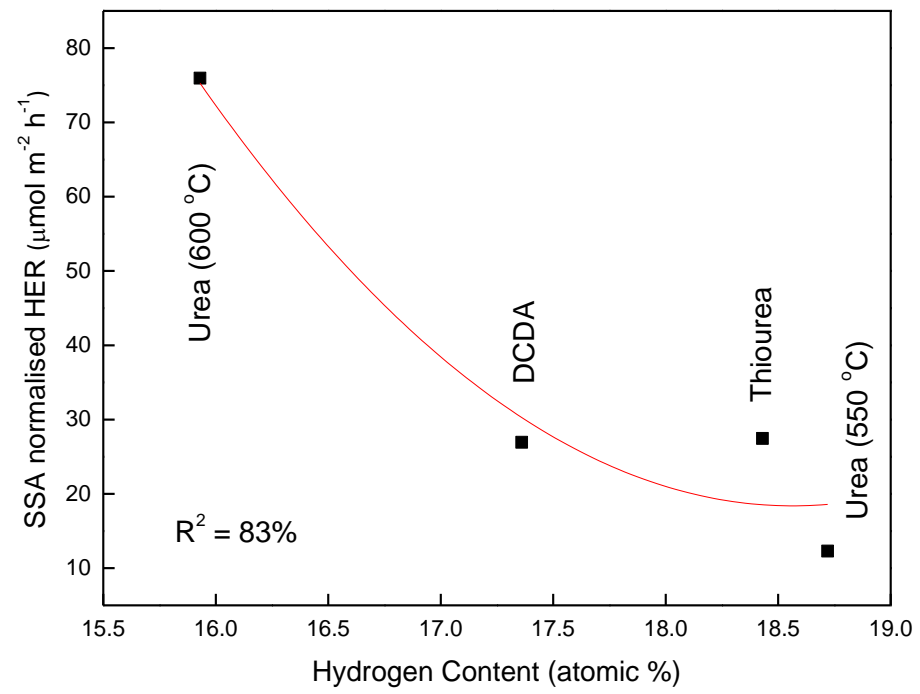

Figure 55. Correlation between specific surface area normalised hydrogen evolution rate and bulk hydrogen content measured by elemental analysis.

EA results showed that DCDA, thiourea and urea $\left(550,600 \& 650{ }^{\circ} \mathrm{C}\right)$ derived $\mathrm{g}_{-} \mathrm{C}_{3} \mathrm{~N}_{4}$ possessed $17.36,18.43,18.72,15.93,15.45$ atom\% of hydrogen respectively (Figure 55). Protons/hydrogen in $\mathrm{g}^{-} \mathrm{C}_{3} \mathrm{~N}_{4}$ is present at $\mathrm{sp}^{3}$ bound nitrogen sites $\left(\mathrm{H}-\mathrm{N}-[\mathrm{C}]_{3}\right)$, and as amino functional groups (C$\mathrm{NH}_{\mathrm{x}}$ ). Proton/hydrogen atomic\% represents bulk protonation status. It was found that the specific surface area $\left(\mathrm{SSA}, \mathrm{m}^{2} \mathrm{~g}^{-1}\right.$ ) normalised hydrogen

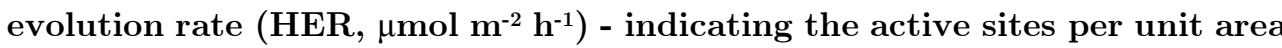
for hydrogen production - is in accordance with the measured hydrogen content. Note that $\mathrm{g}-\mathrm{C}_{3} \mathrm{~N}_{4}$ starts to decomposed above $600{ }^{\circ} \mathrm{C}$; urea $(650$ ${ }^{\circ} \mathrm{C}$ ) derived $\mathrm{g}-\mathrm{C}_{3} \mathrm{~N}_{4}$ is therefore not included in the above comparison. This phenomenon further confirms the relationship between hydrogen evolution and proton concentration.

The increase in protons (from both $\mathrm{sp}^{3}$ nitrogen, and $\mathrm{C}-\mathrm{NH}_{\mathrm{x}}$ ) most likely stems from the route of condensation, and even though all three samples were synthesized using identical conditions, the extent of polymerization varies due to different precursors, which is consistent with the trends shown in XRD and FTIR measurements (Figure 41 \& Figure 42). In particular, only urea-derived $\mathrm{g}-\mathrm{C}_{3} \mathrm{~N}_{4}$ loses hydrogen in the form of formaldehyde, due to the presence of oxygen in the precursor (TGA-DSC-MS; Figure $47 \&$ Figure 48). This trend is not just between different precursors, in fact, urea-derived $\mathrm{g}-\mathrm{C}_{3} \mathrm{~N}_{4}$ synthesized at different temperatures $\left(550,650{ }^{\circ} \mathrm{C}\right)$ also follow suit (Figure 53 \& Table 10); as protonation increases, activity 
decreases. Therefore, both the precursors and synthesis parameters can control the protonation and polymerisation, leading to varying activity. A small change in preparation method can have a huge impact on photocatalytic hydrogen production (such as from $550{ }^{\circ} \mathrm{C}$ to $600{ }^{\circ} \mathrm{C}$ and then to $650{ }^{\circ} \mathrm{C}$; Figure 50(a) and ramp rate changes Figure 50(b)). Previous studies on the protonation status of graphitic carbon nitride have shown that the absorption profile can be shifted by treating compound with $\mathrm{HCl}$, and thus protonating the extrinsic surface structure ${ }^{236}$. Therefore it is reasonable to assume that by altering the synthetic parameters, it is plausible that the intrinsic structure has changed during polymerisation, which could explain the change in absorption (Figure 43) and hydrogen evolution rate from water (Figure 49).

In order to futher determine why polymerisation and protonation status influences $\mathrm{H}_{2}$ production rates, protonation was modelled using DFT simulations with periodic supercells. TDDFT simulations were also performed on smaller cluster models in order to determine the effects of hydrogen on excited state properties.

The density of states (DOS) is shown in Figure 56(c). It can be clearly seen that the CBE of the protonated system is shifted down in energy (towards more positive values with respect to the normal hydrogen electrode (NHE)) by $0.34 \mathrm{eV}$. This significantly modifies electrochemical properties as it provides a lower overpotential for reduction reactions, also shown in the UV-Vis absorption spectra (Figure 43(c)). The reason behind the drop in the position of the CBE can be clearly seen in the site-decomposed DOS, Figure 57. The effects of protonation on excited state properties were also calculated. It was established that the lowest energy vibrationally stable structure is away from a planar configuration. The onset of structure involves strong distortions of all three heptazine rings optical absorption of protonated g- $\mathrm{C}_{3} \mathrm{~N}_{4}$ occurs at a lower energy (more positive with respect to the NHE) than for un-protonated $\mathrm{g}-\mathrm{C}_{3} \mathrm{~N}_{4}$. Indeed, two absorption peaks of the $\mathrm{C}_{18} \mathrm{~N}_{28} \mathrm{H}_{13}$ model occur at lower energies than the initial absorption peak of the $\mathrm{C}_{18} \mathrm{~N}_{28} \mathrm{H}_{12}$ model, in qualitative agreement with the DFT DOS in Figure 56. This verifies the DFT-based electronic structure analysis with TDDFT. 
(a)



(b)

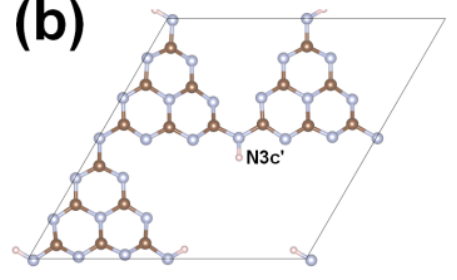

(c)

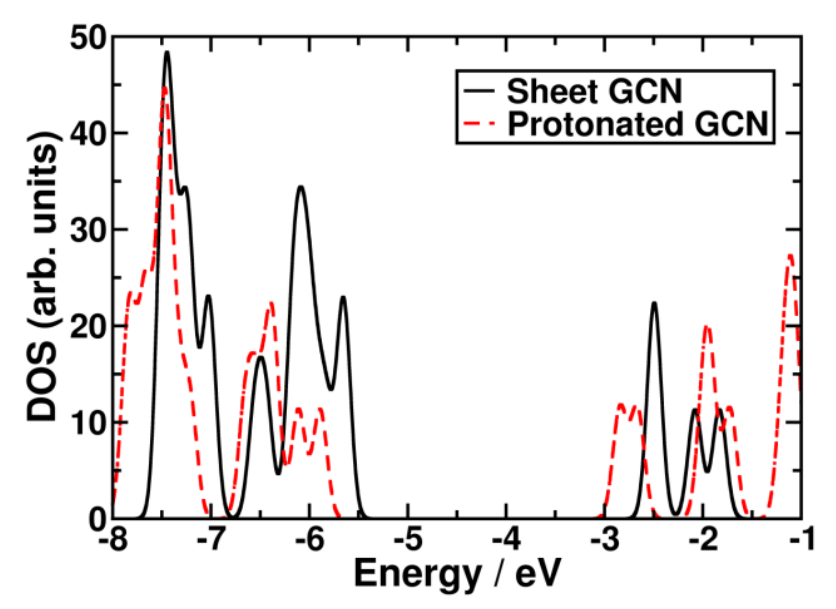

Figure 56. Geometric and electronic structure of single sheet $\mathrm{g}-\mathrm{C}_{3} \mathrm{~N}_{4}$. (a) Supercell model of sheet carbon nitride, (b) supercell model of protonated carbon nitride. Nitrogen is denoted by blue spheres, carbon brown spheres, and hydrogen light pink spheres. (c) Total density of states for sheet carbon nitride (black line) and protonated carbon nitride (red line). Energy is with respect to the zero of the sheet carbon nitride simulation. The DOS of the protonated carbon nitride has been shifted so that the corresponding zero points align.

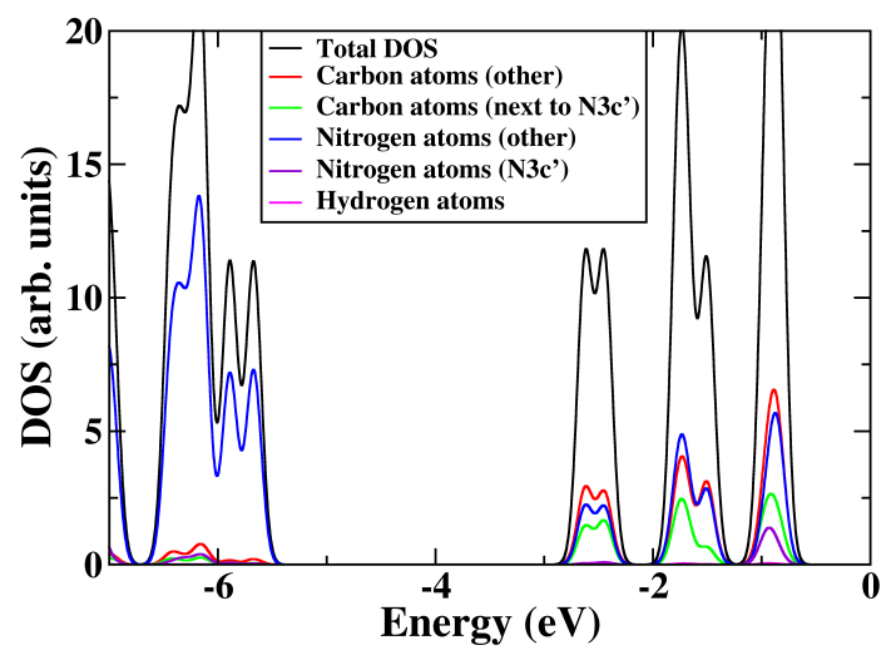


Figure 57. Total and site-decomposed DOS for protonated carbon nitride model. The strong contribution of the carbon atoms adjacent to the N3c' site at the CBE is apparent, as the six atoms contribute almost as much to the DOS as the other twelve carbon atoms in the supercell.

The distribution of the lowest energy exciton for both carbon nitride models was also plotted. The results are shown in Figure 58(b, c). For unprotonated $\mathrm{g}-\mathrm{C}_{3} \mathrm{~N}_{4}$ the exciton is distributed homogeneously over the cluster, with transitions from occupied $\mathrm{N}_{\mathrm{z}}$ orbitals to empty $\mathrm{C} \mathrm{p}_{\mathrm{z}}$ orbitals. For protonated $\mathrm{g}-\mathrm{C}_{3} \mathrm{~N}_{4}$, the exciton is more heterogeneous, with the photohole on the protonated-heptazine ring and the photoelectron distributed evenly on the other two heptazine rings. Although there is a better spatial separation between photohole and photoelectron, both charge carriers are more localized around the central N3 site, and thus are not as available to participate in the photochemical reactions. Moreover, this localisation will act to increase the exciton recombination rate, hindering the efficient utilisation of charge carriers.

\section{(a)}
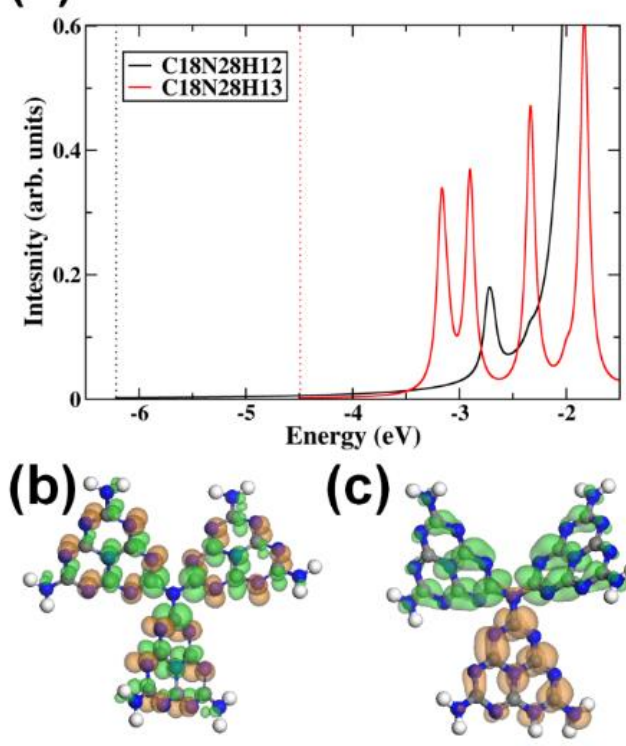

(c)

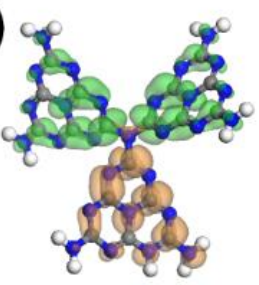

Figure 58. Excited state properties of bare (sheet) and protonated carbon nitride; (a) Optical properties of $\mathrm{C}_{18} \mathrm{~N}_{28} \mathrm{H}_{12}$ (black line) and $\mathrm{C}_{18} \mathrm{~N}_{28} \mathrm{H}_{13}$ (red line) clusters, the $\mathrm{x}$-axis is labeled with respect to the vacuum level $(\mathrm{E}=0)$ while dashed lines indicate the positions of the HOMO states of the two clusters, (b) lowest energy exciton of $\mathrm{C}_{18} \mathrm{~N}_{28} \mathrm{H}_{12}$ cluster, (c) lowest energy exciton of $\mathrm{C}_{18} \mathrm{~N}_{28} \mathrm{H}_{13}$ cluster. Orange isosurface indicates distribution of photohole upon photoexcitation, green isosurface indicates distribution of 
photoelectron upon photoexcitation. Isosurfaces were plotted at 0.005 $|\mathrm{e}| / \AA^{3}$. Blue spheres denote hydrogen atoms, grey spheres denote carbon atoms, white spheres denote hydrogen atoms.

A relatively small change in protonation has a two-pronged detrimental effect on the reduction ability of $\mathrm{g}-\mathrm{C}_{3} \mathrm{~N}_{4}$. Protonation significantly reduces the reduction potential and also localizes excitons around a central nitrogen N3 site, hindering migration to active sites. Here, protonation is essentially controlled through the degree of polymerisation, but also coupled with the degree of condensation. Graphitic carbon nitride, if "under-polymerised" (at low temperatures), has incomplete heptazine coupling, which results in excess hydrogen passivating N3c' nitrogen sites, hampering activity. If "over-polymerised" (at temperatures ca. $650{ }^{\circ} \mathrm{C}$ ), the structure of $\mathrm{g}^{-} \mathrm{C}_{3} \mathrm{~N}_{4}$ tends to overly condense into buckled multi-layered crystals of reduced surface area and hence reduced density of active sites, adversely effecting photocatalytic ability. Moreover, the buckling also distorts the $\mathbf{s p}^{2}$ planar geometry, leading to charge trapping states at nitrogen sites and hence reduced activity.

Table 12. Comparison of typical g- $\mathrm{C}_{3} \mathrm{~N}_{4}$ photocatalysts reported for hydrogen production and the corresponding quantum yields.

\begin{tabular}{|c|c|c|c|c|}
\hline Photocatalyst* & $\begin{array}{c}\text { Band } \\
\text { gap } \\
(\mathrm{eV})\end{array}$ & $\begin{array}{c}\text { HER rate } \\
\text { under visible } \\
\text { light }\left(\mu \mathrm{mol} \mathrm{hr}^{-}\right. \\
\left.{ }^{1} \mathrm{~g}^{-1}\right)^{* *}\end{array}$ & $\begin{array}{c}\text { IQY, \% } \\
\text { (band pass } \\
\text { filter, } \mathrm{nm} \text { ) }\end{array}$ & Reference \\
\hline $\begin{array}{c}\mathrm{g}-\mathrm{C}_{3} \mathbf{N}_{4} \\
\text { (cyanamide) }\end{array}$ & 2.7 & 106 & $\begin{array}{c}\text { ca. } 0.1(420- \\
460 \mathrm{~nm})\end{array}$ & 164 \\
\hline mpg- $\mathrm{C}_{3} \mathrm{~N}_{4}$ & 2.7 & 1490 & N/A & 162 \\
\hline g- $\mathrm{C}_{3} \mathrm{~N}_{4}$ (S-doped) & 2.85 & 800 & $\mathbf{N} / \mathbf{A}$ & 160 \\
\hline $\begin{array}{c}\text { g- } \mathrm{C}_{3} \mathrm{~N}_{4} \\
\text { nanosheets }\end{array}$ & 2.35 & 1860 & $3.7(420 \mathrm{~nm})$ & 165 \\
\hline $\begin{array}{c}\mathrm{g}-\mathrm{C}_{3} \mathrm{~N}_{4}(\mathrm{MCA}- \\
\mathrm{DMSO})\end{array}$ & 2.83 & $\mathrm{~N} / \mathrm{A}$ & $2.3(420 \mathrm{~nm})$ & 228 \\
\hline
\end{tabular}




\begin{tabular}{|c|c|c|c|c|}
\hline $\begin{array}{c}\text { Our } \mathbf{g}-\mathbf{C}_{3} \mathbf{N}_{4} \\
\text { (cyanamide) }\end{array}$ & 2.7 & 142 & N/A & $\begin{array}{c}\text { This } \\
\text { work }\end{array}$ \\
\hline $\begin{array}{c}\text { Our } \mathbf{g}-\mathbf{C}_{3} \mathbf{N}_{4} \\
\text { (MCA-DMSO) }\end{array}$ & 2.8 & 261 & $3.1(400 \mathrm{~nm})$ & $\begin{array}{c}\text { This } \\
\text { work }\end{array}$ \\
\hline $\begin{array}{c}\text { Our g- } \mathbf{C}_{3} \mathbf{N}_{4} \\
\text { (urea) }\end{array}$ & 2.85 & $\mathbf{3 3 2 7}$ & $\begin{array}{c}\mathbf{2 6 . 5}(\mathbf{4 0 0} \\
\mathbf{n m})\end{array}$ & $\begin{array}{c}\text { This } \\
\text { work }\end{array}$ \\
\hline
\end{tabular}

*All photocatalysts are loaded with $3 \mathrm{wt} \% \mathrm{Pt}$ co-catalysts except g- $\mathrm{C}_{3} \mathrm{~N}_{4}$ (Sdoped) which is mixed with $6 \mathrm{wt} \% \mathrm{Pt}$ co-catalyst. ${ }^{* *} \mathrm{HER}$ rates are measured either by using long pass $(\lambda \geq 420 \mathrm{~nm})$ or $(\lambda \geq 395 \mathrm{~nm})$ such as for our $\mathrm{g}-\mathrm{C}_{3} \mathrm{~N}_{4}$ (cyanamide) and our $\mathrm{g}-\mathrm{C}_{3} \mathrm{~N}_{4}$ (urea), therefore the small difference is due to the long pass filters used.

As mentioned in section 2.3.2, apart from a photocatalyst needing to be cheap and robust, it must exhibit a high quantum yield for hydrogen production from water under visible light if it is even to be considered commercially viable. Compounds that traditionally exhibit high efficiencies either suffer from instability (e.g. sulphides ${ }^{101,239}$ ) or are made of relatively expensive metals (e.g. GaAs-GaInP ${ }_{2}^{68}$ ). It is shown in Figure 59(a) the cheap and stable urea-derived $\mathrm{g}-\mathrm{C}_{3} \mathrm{~N}_{4}$ has a peak internal quantum yield of $28.4 \%$ at $365 \mathrm{~nm}$. Even under visible light irradiation at $\lambda=400 \mathrm{~nm}$, the quantum yield is $26.5 \%$, nearly an order of magnitude greater than the highest reported $\left(3.75 \%\right.$ at $420 \mathrm{~nm}^{[7 f]}$, obtained via liquid exfoliation). In order to assure the reliability of our measurement, we repeated a benchmark cyanamide derived $\mathrm{g}_{-} \mathrm{C}_{3} \mathrm{~N}_{4}$ as a reference which showed comparable activity (small difference is due to a $395 \mathrm{~nm}$ long pass filter used instead of a $420 \mathrm{~nm}$ filter).

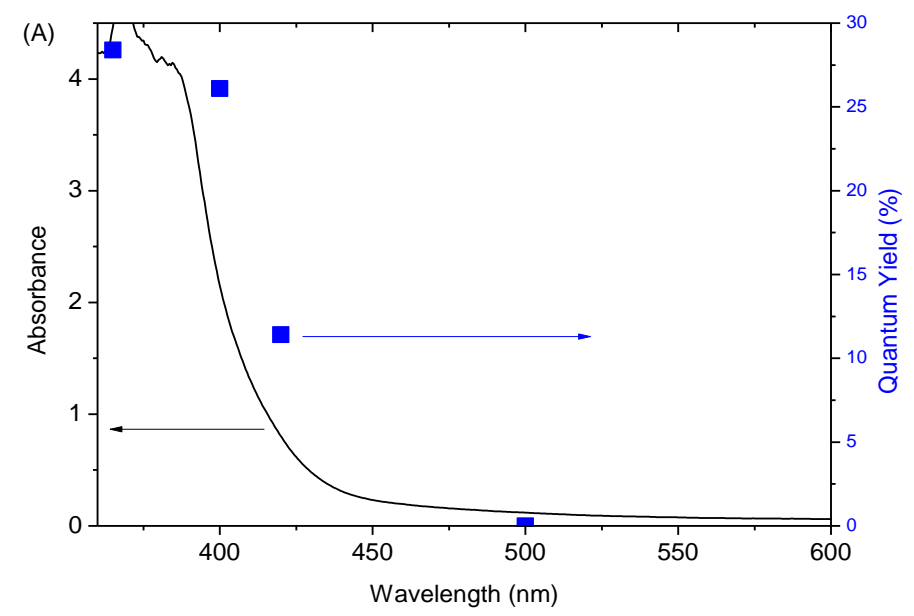




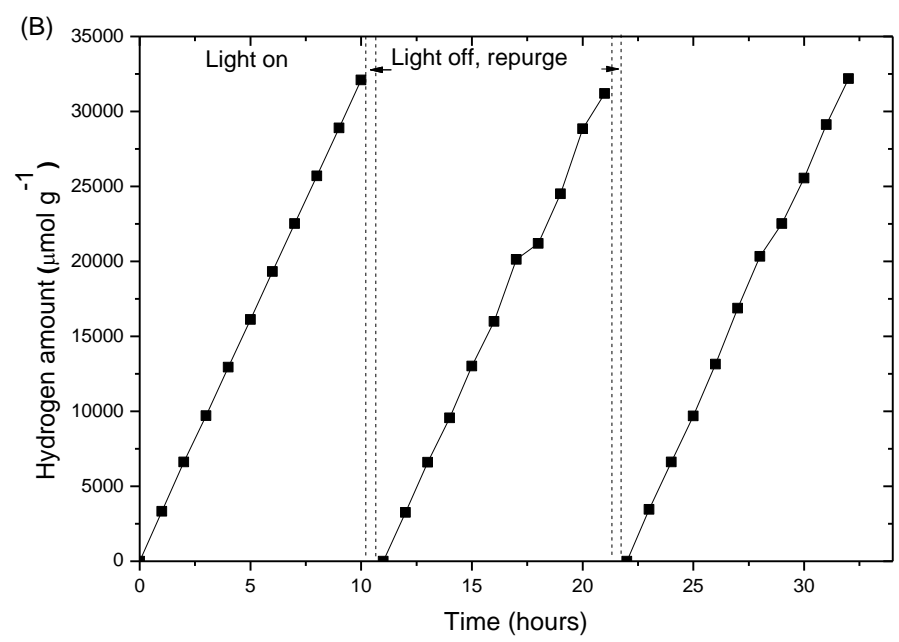

Figure 59. (a) Quantum yield of urea based $\mathrm{g}-\mathrm{C}_{3} \mathrm{~N}_{4}$; using band pass filters at specific wavelengths (black line represents absorbance, internal quantum yield is shown by blue points). (b) Stability test of the urea derived g- $\mathrm{C}_{3} \mathrm{~N}_{4}$ under visible light irradiation $(\lambda \geq 395 \mathrm{~nm})$.

As proposed previously, the huge enhancement in hydrogen evolution rate of our urea-derived sample $\left(3327.5 \mu \mathrm{mol} \mathrm{hr}{ }^{-1} \mathrm{~g}^{-1} \mathrm{vs} .142 .3 \mu \mathrm{mol} \mathrm{hr} \mathrm{h}^{-1} \mathrm{~g}^{-1}\right)$ can be attributed to a lower protonation status and condensation state. Very recently, a facile synthesis method for three-dimensional porous $\mathrm{g}^{-} \mathrm{C}_{3} \mathrm{~N}_{4}$ was introduced by using the aggregates of melamine and cyanuric acid (MCA) co-crystals in dimethyl sulfoxide (DMSO, sample denoted MCA_DMSO) as precursors ${ }^{228}$. It was reported the quantum yield of $\mathrm{g}-\mathrm{C}_{3} \mathrm{~N}_{4}$ MCA_DMSO at $\lambda=420 \mathrm{~nm}$ was $2.3 \%$ (Table 12 ), much higher than the melamine derived bulk $\mathrm{g}-\mathrm{C}_{3} \mathrm{~N}_{4}(0.26 \%)$ under the same conditions. This study was repeated, and as shown in Figure 60, very similar morphologies and optical properties compared with the literature were measured. A similar quantum yield of $3.1 \%$ at $\lambda=400 \mathrm{~nm}$ (Table 12) was obtained (difference is due to the wavelength of band-pass filter). Since MCA_DMSO is another oxygencontaining precursor, the rise in the quantum yield compared with a melamine sample further supports the proposed protonation mechanism. In addition, a theory as to why the optimised urea derived g- $\mathrm{C}_{3} \mathrm{~N}_{4}$ demonstrates a tenfold increase in IQY yield at $400 \mathrm{~nm}$, in comparison to $\mathrm{MCA}_{-}{ }^{-D M S O} \mathrm{~g}-\mathrm{C}_{3} \mathrm{~N}_{4}$, is because of the much higher oxygen concentration in the urea precursor, which helps to passivate protonation sites and polymerise g- $\mathrm{C}_{3} \mathrm{~N}_{4}$ without structural instability/buckling. 


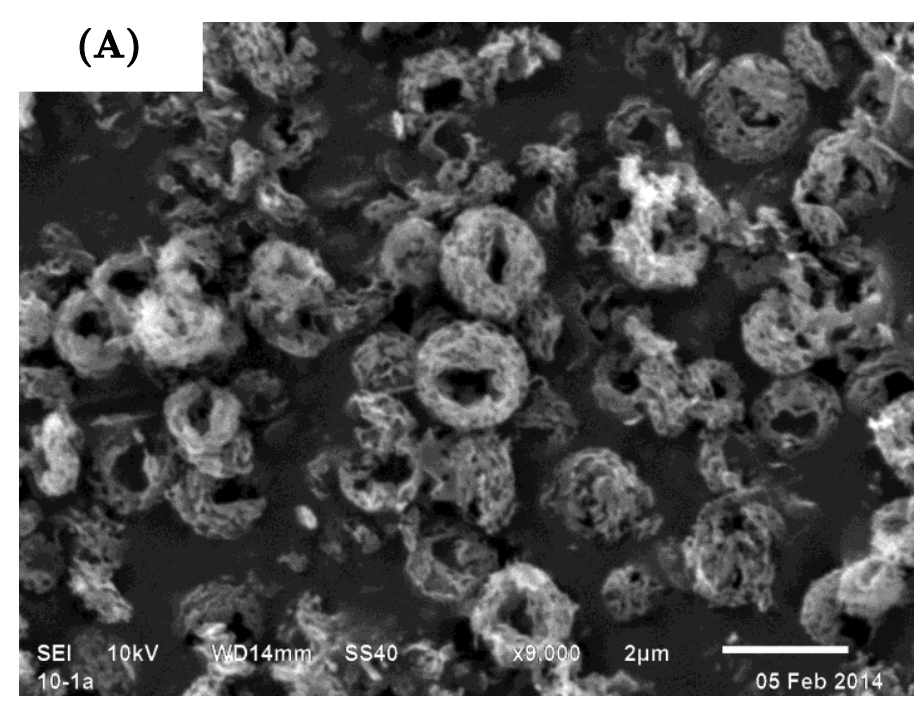

(B)

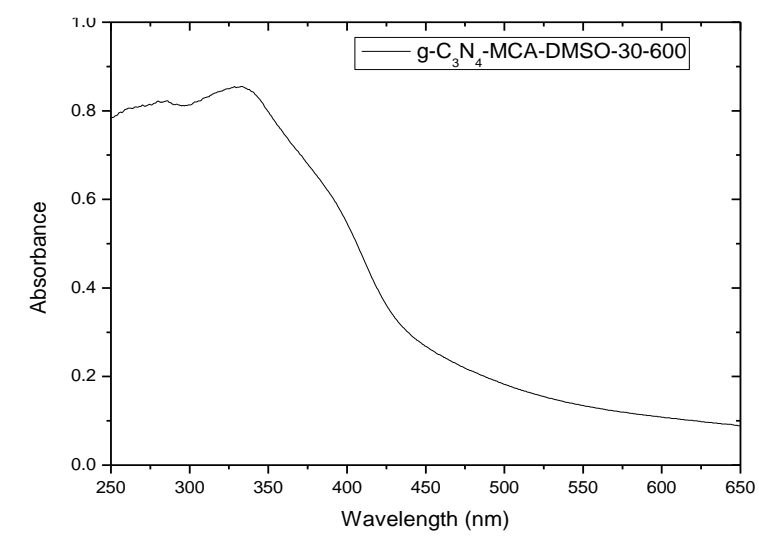

Figure 60. (A) SEM micrograph of g- $\mathrm{C}_{3} \mathrm{~N}_{4}$-MCA-DMSO-30-600. (B) UVVis absorbance spectra of $\mathrm{g}-\mathrm{C}_{3} \mathrm{~N}_{4}$-MCA-DMSO-30-600.

The stability of the optimised photocatalyst was also tested in an extended experiment, as illustrated in Figure 59(b). The high activity is reproducible and the material shows excellent stability within a 30 hour run. Attributes for a high quantum yield commonly include good absorption, efficient charge separation and rapid carrier transfer to the surface for redox reactions. Even though the overall band gap of urea based carbon nitride is larger than that of both DCDA and thiourea derived carbon nitrides, it produces more hydrogen, therefore the bulk absorption and band gap magnitude are not the determining factor in overall activity. Due to the fewer protons in $\mathrm{g}-\mathrm{C}_{3} \mathrm{~N}_{4}$ (urea), the bandgap and therefore the overpotential is larger, which also results in better separation of charge, and consequently causes better migration of charger carriers to active sites. The trend of the proton concentration and the trend of polymerisation for the sample set are 
in very good agreement with the corresponding activity, which contributes to charge transport. Therefore for the first time, it has been demonstrated that the protonation and degree of polymerisation determines hydrogen evolution rates from water, with surface area playing a minor role ${ }^{227}$.

\subsection{Conclusions}

To conclude, a novel strategy for synthesising graphitic carbon nitride has been demonstrated, by thermally decomposing urea at appropriate temperature (e.g $\left.600{ }^{\circ} \mathrm{C}\right)$, ramp rate $\left(5^{\circ} \mathrm{C}\right)$ and hold time (4 hours). Several different precursors were used, including urea, thiourea, and DCDA. XRD patterns exhibit varying peak intensities indicating the most crystallised/condensed sample is DCDA, and urea the least, as a more intense peak implies that there is more regular repetitions between graphitic layers. UV-Vis spectra shows the sample synthesised from thiourea shows the largest absorption, with an edge around $455 \mathrm{~nm}$. The DCDA derived sample absorbs up to ca. $450 \mathrm{~nm}$, whilst the sample fabricated using urea exhibited a visible blue shift, absorbing light up to approximately $420 \mathrm{~nm}$. All the samples have a small absorption tail, due to some sub band gap states from $n-\pi^{*}$ transitions. TGA-DSC-MS data shows that as urea based $\mathrm{g}-\mathrm{C}_{3} \mathrm{~N}_{4}$ is thermally decomposed, $\mathrm{CO}_{2}$ is given off in large amounts, and up to the polymerisation point of heptazine units (over 500 $\left.{ }^{\circ} \mathrm{C}\right)$. The presence of $\mathrm{CO}_{2}$ is crucial for the formation of the largest pores and the highest surface area - and is not present in either the decomposition of thiourea or DCDA. Furthermore, formaldehyde is also given off throughout decomposition, and continually removes hydrogen from the structure, which helps to promote polymerisation.

The internal quantum yield in the visible region by the optimised sample is $26.5 \%$, nearly an order of magnitude higher than reported previously. Under full arc irradiation, the optimized $\mathrm{g}-\mathrm{C}_{3} \mathrm{~N}_{4}$ can be tailored to produce ca. $20,000 \mu \mathrm{mol} \mathrm{g}{ }^{-1}$ of hydrogen per hour from water. The optimized $\mathrm{g}-\mathrm{C}_{3} \mathrm{~N}_{4}$ photocatalyst is very stable, exhibiting a near linear profile of $\mathrm{H}_{2}$ production from water for 30 hours and resulting in a TON of over 641 in terms of $\mathrm{Pt}$ cocatalyst after a 6 hour test under 300W Xe lamp irradiation. Data from XPS, FTIR, zeta potential and XRD show that both protonation status and degree of polymerisation can influence the $\mathrm{g}-\mathrm{C}_{3} \mathrm{~N}_{4}$ hydrogen evolution rate. 
Specifically, from the N1s core XPS data, we observe the ratio of $\mathrm{sp}^{2} \mathrm{C}-\mathrm{N}$ $\mathrm{C}$ to the sum of $\mathrm{sp}^{3} \mathrm{H}-\mathrm{N}-[\mathrm{C}]_{3}$ and $\mathrm{C}-\mathrm{NH}_{\mathrm{x}}$ bonds (where the latter represents the total amount of protons) is 2.83 in urea, 2.7 for thiourea and only 2.31 in DCDA. As it is part of the heptazine ring, linked by a double and a single bond to two opposing carbons, the $\mathrm{sp}^{2}$ bonded nitrogen is the principle participant that contributes to band gap absorption and therefore is an incredibly important part of the structure. Both hybridized $\mathbf{s p}^{3}$ nitrogen and surface functional amino groups $\left(\mathrm{C}-\mathrm{NH}_{\mathrm{x}}\right)$ are also key features when considering bulk and surface properties. Along with $\mathrm{C}-\mathrm{NH}_{\mathrm{x}}$ bonding, graphitic carbon nitride possesses a positively charged, slightly acidic surface, confirmed by zeta potential measurements. Elemental analysis (EA) further confirms the trend; as bulk $\mathrm{H}$ (at\%) increases, the HER per SSA $\left(\mu \mathrm{mol} \mathrm{m} \mathrm{m}^{-2} \mathrm{~h}^{-1}\right)$ decreases. Combining with XPS analysis, one can conclude that a lower proton concentration leads to a larger HER.

Two different lines of computational evidence (DFT and TDDFT) have shown that the larger HER is a direct result of a decrease in surface protonation status. This is because of a shift in the position of the conduction band edge occurs, thus increasing the overpotential for reduction reactions at the surface. A larger overpotential allows reduction reactions to proceed more readily, despite increasing the band gap. Furthermore, there is a significant shift in the CBE position even for limited proton concentrations that are less than experimental values $(6.5 \%$ atomic $H$ for DFT, and $1.6 \%$ atomic $H$ for TDDFT, versus $15.5 \%$ atomic $H$ evidenced by EA). Excess protonation also localizes photoelectrons at nonactive N3 redox sites, effectively rendering the photoexcited charge useless.

The evidence contained in this chapter suggests that not only does urea based $\mathrm{g}-\mathrm{C}_{3} \mathrm{~N}_{4}$ exhibit a much larger efficiency in comparison to other samples, but also that this is a direct result of the band structure and surface chemistry. Therefore considering this enhancement and the energetic requirements of Z-Scheme systems, the next chapter will focus on the incorporation of $\mathrm{g}^{-} \mathrm{C}_{3} \mathrm{~N}_{4}$ into a Z-Scheme system, a never before explored experiment due to the previously reported low quantum yields of carbon nitride at visible wavelengths. 


\section{Novel Z-Scheme overall water splitting systems}

Following the success of an extremely efficient photocatalyst for both $\mathrm{O}_{2}$ and $\mathrm{H}_{2}$ evolution under visible light, the research focus naturally moved to a demonstration system for pure water splitting by using urea derived g$\mathrm{C}_{3} \mathrm{~N}_{4}$ and tetrahedral $\mathrm{Ag}_{3} \mathrm{PO}_{4}$ crystals. It was found $\mathrm{Ag}_{3} \mathrm{PO}_{4}$ was also not able to function as an oxygen evolving photocatalyst in $\mathrm{Z}$-scheme water splitting systems due to the incompatibility between photocatalyst and synthetic conditions. $\mathrm{Ag}_{3} \mathrm{PO}_{4}$ dissolves into the solution at the $\mathrm{pH}$ range ( $\leq$ 3.5) required for $\mathrm{Fe}^{2+/ 3+}$ to be an effective redox mediator, and also partially reacts with $\mathrm{H}_{2} \mathrm{SO}_{4}$ to form $\mathrm{Ag}_{2} \mathrm{SO}_{4}-\mathrm{Ag}_{3} \mathrm{PO}_{4}$ as evidenced by XRD data. When $\mathrm{NaI}$ is used as a redox mediator, TEM and EDX results show that $\mathrm{Ag}_{3} \mathrm{PO}_{4}$ reacts with the iodide anion to form AgI, visibly changing the colour of the solution before illumination and also demonstrating no activity for overall water splitting.

Urea derived $\mathrm{g}-\mathrm{C}_{3} \mathrm{~N}_{4}$ is shown herein to actively participate in novel Zscheme water splitting systems as a hydrogen evolving photocatalyst. Due to the robustness and favourable band position, g- $\mathrm{C}_{3} \mathrm{~N}_{4}$ can be coupled with either $\mathrm{I}^{-} / \mathrm{IO}_{3}{ }^{-}$or $\mathrm{Fe}^{2+/ 3+}$ redox mediators, at any $\mathrm{pH}$, and with Pt-loaded $\mathrm{WO}_{3}$ or $\mathrm{BiVO}_{4}$ respectively. The highest water splitting rates were achieved using a g- $\mathrm{C}_{3} \mathrm{~N}_{4}-\mathrm{NaI}-\mathrm{WO}_{3}$ system, peaking at 36 and $18 \mu \mathrm{mol} \mathrm{h}^{-1} \mathrm{~g}^{-1}$ of hydrogen and oxygen respectively. The largest $\mathrm{STH} \%$ was recorded at $0.1 \%$.

The redox sensitivity of the $\mathrm{WO}_{3}\left(\mathrm{I} / \mathrm{IO}_{3}{ }^{-}\right)$water splitting system was demonstrated by testing $\mathrm{WO}_{3}$ platelets, which have a smaller band gap than their commercial counterparts. The narrowing of the band gap, a result of morphological changes, was shown to have a dramatic negative effect upon the system; no water splitting was observed. Carbon nitride synthesized using precursors such as DCDA or thiourea do not give detectable activity for any Z-scheme water splitting reaction. It is postulated that due to the low efficiency of these carbon nitrides in comparison to urea-derived $\mathrm{g}-\mathrm{C}_{3} \mathrm{~N}_{4}$ for hydrogen evolution (as described in chapter 5), overall water splitting rates are undetectable. 


\subsection{Introduction}

A single photocatalyst for overall water splitting has been explored for several decades but met with little success ${ }^{133}$. Nature splits water into $\mathrm{O}_{2}$ and the $\mathrm{H}_{2}$ equivalent species by a double excitation process including PSII and PSI, instead of a single excitation, in which the two half reactions are spatially separated and take place in PSII and PSI, Figure $\mathbf{1 2}^{240}$. This overcomes the main problems of a singular photocatalytic water splitting system both kinetically and thermodynamically, as well described in previous reviews ${ }^{171}$. Inspired by natural photosynthesis, Bard proposed an analogy composed of two inorganic semiconductor photocatalysts in 1979 and recently there have been some productive systems based upon either Rh doped $\mathrm{SrTiO}_{3}$ or $\mathrm{TaON}^{169,170}$.

The ideal artificial double excitation process is illustrated in Figure 12. Briefly, a combination of two semiconductor photocatalysts, in which each photocatalyst is responsible for one half reaction and a soluble mediator helps electron transfer between the two photocatalysts so that an ideal cycle can be completed. A mediator is vital because it dramatically inhibits the fast unfavourable recombination of charge, analogous to the electron transport chain between PSII and PSI. Given such advantages of a double excitation process, there are many researchers working on either two photocatalysts each of which favours either $\mathrm{H}_{2}$ or $\mathrm{O}_{2}$ production, or a new mediator to efficiently transfer the charges between two photocatalysts. Thus a nature-inspired double excitation system composed of abundant elements and working efficiently for overall water splitting is highly soughtafter.

\subsection{Methodology}

\subsubsection{Photocatalytic analysis}

All reactions were carried out in a custom $\mathrm{Pyrex}^{\circledR}$ batch reactor cell, which was thoroughly purged with argon prior to radiation. Gas concentration analysis was performed using a GC (Varian 430-GC, TCD, argon carrier gas $99.999 \%$ ). Platinum, acting as a cocatalyst, was deposited onto g- $\mathrm{C}_{3} \mathrm{~N}_{4}$ using an in situ photodeposition method. Stock solutions of deionised water and precursors (e.g. Pt: $\mathrm{H}_{2} \mathrm{PtCl}_{6} \cdot\left(\mathrm{H}_{2} \mathrm{O}\right)_{6}$ ) were made beforehand, and simply added according to the required weight of metal (various wt\% was tested previously, $3 \mathrm{wt} \%$ is optimum). The reactor was sealed, purged with Ar gas 
for 1 hour, and then irradiated for 1 hour under full arc irradiation using a 300 W Xe lamp (TrusTech PLS-SXE 300/300UV). After one hour, a measurement was taken to determine if hydrogen had been produced, and thus, if photodeposition had occurred correctly. The photocatalyst was then collected, washed three times, and dried overnight.

Platinum was deposited onto $\mathrm{WO}_{3}$ using an impregnation method. $\mathrm{WO}_{3}$ powder was put in an aqueous solution of $\mathrm{H}_{2} \mathrm{PtCl}_{6} \cdot\left(\mathrm{H}_{2} \mathrm{O}\right)_{6}$, and heated until all water had evaporated. The powder was then heated at $500{ }^{\circ} \mathrm{C}$ for 1 hour. After appropriate cocatalysts had been deposited, photocatalysts were immersed in an aqueous redox mediator solution, with different concentrations. The system was sealed, purged with argon gas for 1 hour to remove all air in solution and headspace. A baseline measurement was taken, and then the reactor was irradiated with a $300 \mathrm{~W}$ Xe lamp (TrusTech PLS-SXE 300/300UV). A $395 \mathrm{~nm}$ long pass filter was used to remove UV radiation (Comar Optics) where necessary.

Light intensity measured at 5 different points to obtain an average intensity. The solar to hydrogen efficiency was then calculated using the following formula; STH\% $=$ (Gibbs free energy of water $\times$ hydrogen production rate / energy density of incoming irradiation $\times$ area of light beam) $\times 100$.

\subsubsection{Synthesis techniques}

Highly efficient graphitic carbon nitride was synthesised using urea (SigmaAldrich $99.9 \%$ ). In a typical run, urea was placed in a lidded high quality alumina crucible, then put inside a muffle furnace and heated to $600{ }^{\circ} \mathrm{C}$ for several hours $\left(5{ }^{\circ} \mathrm{C} \mathrm{min}^{-1}\right.$ ramp rate). The whole procedure was then carefully optimised to give the highest activity for $\mathrm{H}_{2}$ production from water, as previously described in chapter 5 . Conventional photoactive monoclinic $\mathrm{WO}_{3}$ was purchased directly from a supplier (Sigma-Aldrich) and further ground into a fine powder. $\mathrm{BiVO}_{4}$ was synthesised using a method described by Kudo et $\mathrm{al}^{17}$. Monoclinic $\mathrm{WO}_{3}$ platelets were synthesised using a facile recipe tailored in house, based on a classical fabrication route to synthesise non-faceted monoclinic $\mathrm{WO}_{3}{ }^{241}$. The classical route uses $\mathrm{CaWO}_{4}$ as the precursor, which is not soluble in water. Here $\mathrm{Na}_{2} \mathrm{WO}_{4} \cdot 2 \mathrm{H}_{2} \mathrm{O}$ is dissolved (1g, Alfa Aesar $99.8 \%$ ) in $50 \mathrm{~cm}^{3} \mathrm{DI}$ water, after which $5 \mathrm{M} \mathrm{HCl}$ was added dropwise until a green $\mathrm{H}_{2} \mathrm{WO}_{4}$ precipitate 
appeared. This was then stirred for $\mathbf{2 4}$ hours, washed with DI water, and filtered. The precipitate was then dried overnight and subsequently placed into a lidded alumina crucible, and calcined in a muffle furnace at $500{ }^{\circ} \mathrm{C}$ for 6 hours, with a ramp rate of $10{ }^{\circ} \mathrm{C} \mathrm{min}{ }^{-1}$. The $\mathrm{WO}_{3}$ platelets were then ground into a fine powder. 


\subsection{Results and discussion}

6.3.1 $\mathrm{Ag}_{3} \mathrm{PO}_{4}$ based $\mathrm{Z}$-scheme water splitting systems

Attempts were made to utilise $\mathrm{Ag}_{3} \mathrm{PO}_{4}$ in Z-Scheme system, coupled with g- $\mathrm{C}_{3} \mathrm{~N}_{4}$. Tetrahedral silver phosphate, synthesised using Method ' $\mathrm{D}$ ' in chapter 4 immersed first (before $\mathrm{g}-\mathrm{C}_{3} \mathrm{~N}_{4}$ ) in an aqueous $2 \mathrm{mM} \mathrm{Fe}^{2+/ 3+}$ (starting with $\mathrm{FeCl}_{3}$ ) redox mediator solution. $\mathrm{H}_{2} \mathrm{SO}_{4}$ was used to adjust the pH to 3, at which point $\mathrm{Ag}_{3} \mathrm{PO}_{4}$ completely dissolved into the solution, and thus the experiment was forced to stop. At pH 4, which is essentially the upper $\mathrm{pH}$ limit at which $\mathrm{Fe}^{2+/ 3+}$ does not undergo hydrolysis, $\mathrm{Ag}_{3} \mathrm{PO}_{4}$ appeared stable in the solution. However, a slight discolouration of the suspension was cause for concern. The particles were removed from the reactor, washed, and centrifuged 3 times. After which, XRD results (Figure 61) confirm that $\mathrm{Ag}_{3} \mathrm{PO}_{4}$ indeed did react with $\mathrm{H}_{2} \mathrm{SO}_{4}$ to form an $\mathrm{Ag}_{3} \mathrm{PO}_{4^{-}}$ $\mathrm{Ag}_{2} \mathrm{SO}_{4}$ intermediate. The powder was dispersed and tested alongside g$\mathrm{C}_{3} \mathrm{~N}_{4}$, in a $2 \mathrm{mM} \mathrm{FeCl}_{3}$ solution; at which point water splitting did not occur. Therefore it was concluded $\mathrm{Ag}_{3} \mathrm{PO}_{4}$ was not suitable for a water splitting reaction in a $\mathrm{Fe}^{2+/ 3+}$ mediator.

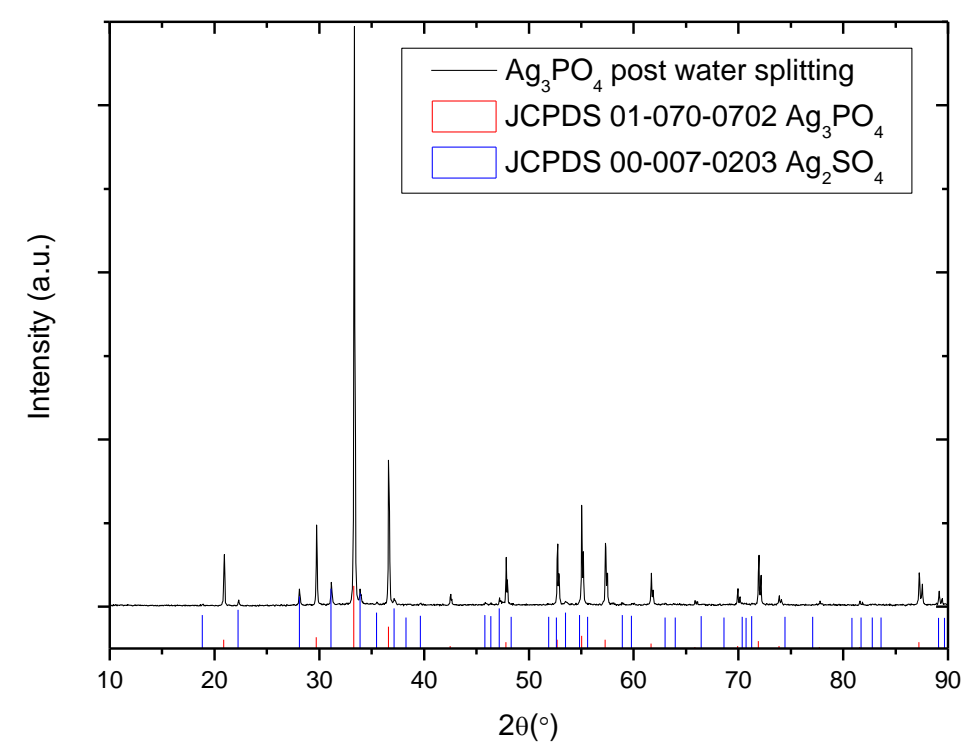

Figure 61. Powder $\mathrm{X}$-ray diffraction data post water splitting in $\mathrm{g}-\mathrm{C}_{3} \mathrm{~N}_{4}-$ $\mathrm{Fe}^{2+/ 3+}-\mathrm{Ag}_{3} \mathrm{PO}_{4}$ system. Both main phase silver phosphate and sub phase silver sulphate are indicated by red and blue bars respectively.

$\mathrm{Ag}_{3} \mathrm{PO}_{4}$ was subsequently tested in system where $\mathrm{NaI}\left(\mathrm{I}^{-} / \mathrm{IO}_{3}^{-}\right)$acted as the redox mediator, and $\mathrm{g}-\mathrm{C}_{3} \mathrm{~N}_{4}$ was the hydrogen production photocatalyst. A 
$5 \mathrm{mM} \mathrm{NaI}$ aqueous solution prepared, and tetrahedral $\mathrm{Ag}_{3} \mathrm{PO}_{4}$ immersed in the electrolyte. The $\mathrm{pH}$ was monitored, and increased from 8.3, to 10.5; at which point the colloid began to change colour from bright yellow, to a light grey/brown. After attempting to collect the spent $\mathrm{Ag}_{3} \mathrm{PO}_{4}$, only a tiny amount was recoverable - the remaining dissolved in the solution. The recovered powder was too little in volume for XRD, so TEM-EDX was performed in order to ascertain the powders nature.

Figure 62 (A) shows a small crystalline region of AgI, comprising of two visible atomic spacings, 0.27 and $0.37 \mathrm{~nm}$, corresponding to the (002) and (101) crystal diffraction planes of wurtzite AgI (P63mc, JCPDS 78-1614). The equivalent FFT (Figure 62(B)) also shows 'diffraction' spots at the same inverse distances (see Table 13 for conversion).
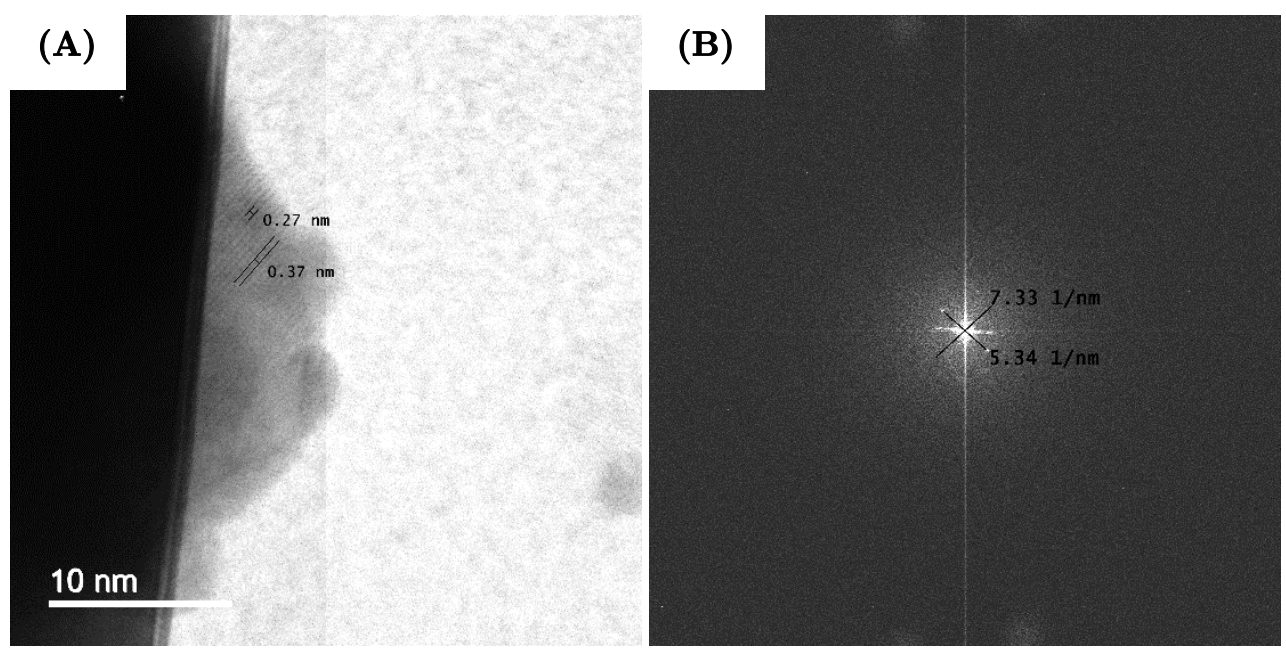

Figure 62. (A) TEM micrographs of $\mathrm{Ag}_{3} \mathrm{PO}_{4}$ with $\mathrm{AgI}$ layer post water splitting, in $\mathrm{g}-\mathrm{C}_{3} \mathrm{~N}_{4}-\mathrm{I}^{-} / \mathrm{IO}_{3}{ }^{-}-\mathrm{Ag}_{3} \mathrm{PO}_{4}$ system. Atomic spaces indicated by parallel lines. (B) Corresponding FFT of micrograph, diameter of diffraction spots are indicated. 
Table 13. AgI FFT diameter relation to a real distance.

\begin{tabular}{|c|c|c|c|}
\hline \multicolumn{4}{|c|}{ AgI } \\
\hline $\begin{array}{c}\text { FFT diameter } \\
(1 / \mathrm{nm})\end{array}$ & $\begin{array}{c}\text { FFT radius } \\
(1 / \mathrm{nm})\end{array}$ & $\begin{array}{c}\text { Real distance } \\
(\mathrm{nm})\end{array}$ & $\begin{array}{c}\text { Real distance } \\
(\AA)\end{array}$ \\
\hline 7.32 & 3.66 & & 2.73 \\
\hline 5.33 & 2.67 & 0.27 & 3.75 \\
\hline
\end{tabular}

Figure 63. (A) Shows the atomic spacing of regular $\mathrm{Ag}_{3} \mathrm{PO}_{4}$ on a TEM micrograph; $0.43 \mathrm{~nm}$ corresponding to the (110) crystal plane. Figure 63 (B) is the equivalent FFT of the TEM micrograph, which shows the 'diffraction' spots of the (110) plane. The conversion of inverse length to real space length is shown in Table 14.

\section{(A)}

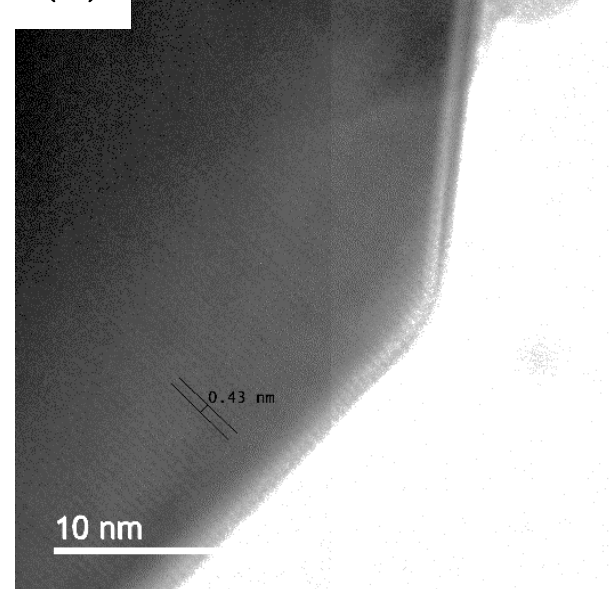

\section{(B)}

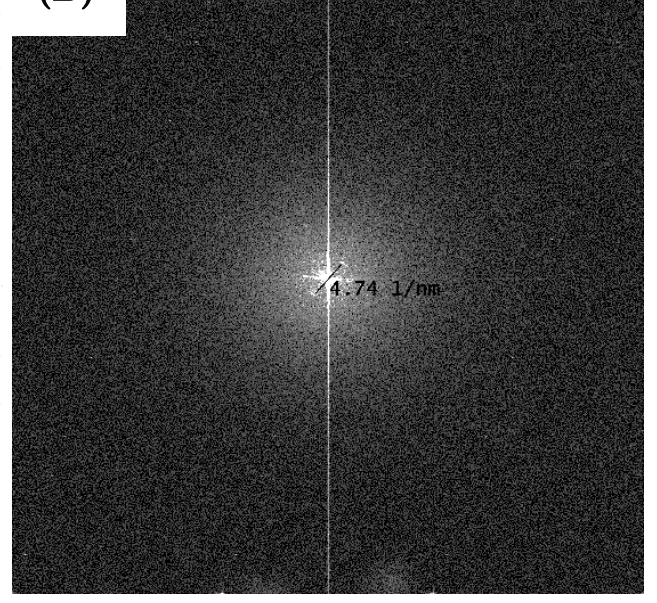

Figure 63. (A) TEM micrograph of an $\mathrm{Ag}_{3} \mathrm{PO}_{4}$ crystal post water splitting, in $\mathrm{g}_{-} \mathrm{C}_{3} \mathrm{~N}_{4}-\mathrm{I}-/ \mathrm{IO}_{3}{ }^{-}-\mathrm{Ag}_{3} \mathrm{PO}_{4}$ system. Atomic plane distance indicated by parallel lines. (B) Corresponding FFT of micrograph, diameter of diffraction spot is indicated. 
Table 14. $\mathrm{Ag}_{3} \mathrm{PO}_{4}$ FFT diameter relation to a real distance.

\begin{tabular}{|c|c|c|c|}
\hline \multicolumn{4}{|c|}{$\mathrm{Ag}_{3} \mathrm{PO}_{4}$} \\
\hline $\begin{array}{c}\text { FFT diameter } \\
\left(\mathrm{nm}^{-1}\right)\end{array}$ & $\begin{array}{c}\text { FFT radius } \\
\left(\mathrm{nm}^{-1}\right)\end{array}$ & $\begin{array}{c}\text { Real distance } \\
(\mathrm{nm})\end{array}$ & $\begin{array}{c}\text { Real distance } \\
(\AA)\end{array}$ \\
\hline 4.700 & 2.350 & 0.426 & 4.255 \\
\hline
\end{tabular}

Figure 64 (A) displays the full elemental spectrum of a $\mathrm{Ag}_{3} \mathrm{PO}_{4}$ - $\mathrm{AgI}$ sample, and Figure 64 (B) illustrates the distribution of elemental Iodine across the sample. From Figure 62, Figure 63, and Figure 64 it is apparent that the AgI layers are both in clumps and well distributed across the $\mathbf{A g}_{3} \mathrm{PO}_{4}$ surface. Whilst not detrimental to the overall structure of $\mathrm{Ag}_{3} \mathrm{PO}_{4}$ particles, AgI layers form quickly, and then are in turn partially reduced (to change from a yellow, to light greg hue) to elemental silver, a process which stops $\mathrm{Ag}_{3} \mathrm{PO}_{4}$ from absorbing light, and halts photocatalytic activity. The ionexchange of phosphate ions into the solution with iodine ions could also be the reason that the $\mathrm{pH}$ increase towards more basic values and increases the solubility of $\mathrm{Ag}_{3} \mathrm{PO}_{4}$, which could be why very little is recoverable.

Ignoring copper and other elements denoted as artefacts $(0 \%$ weight but show up in the spectrum regardless), Figure 64 (C) shows that by weight, there is a significant amount of iodine in the sample. As iodine has a large atomic weight, this value (ca. 12\%) in terms of molar amount is obviously skewed, and would be much lower (ca. 4\%).
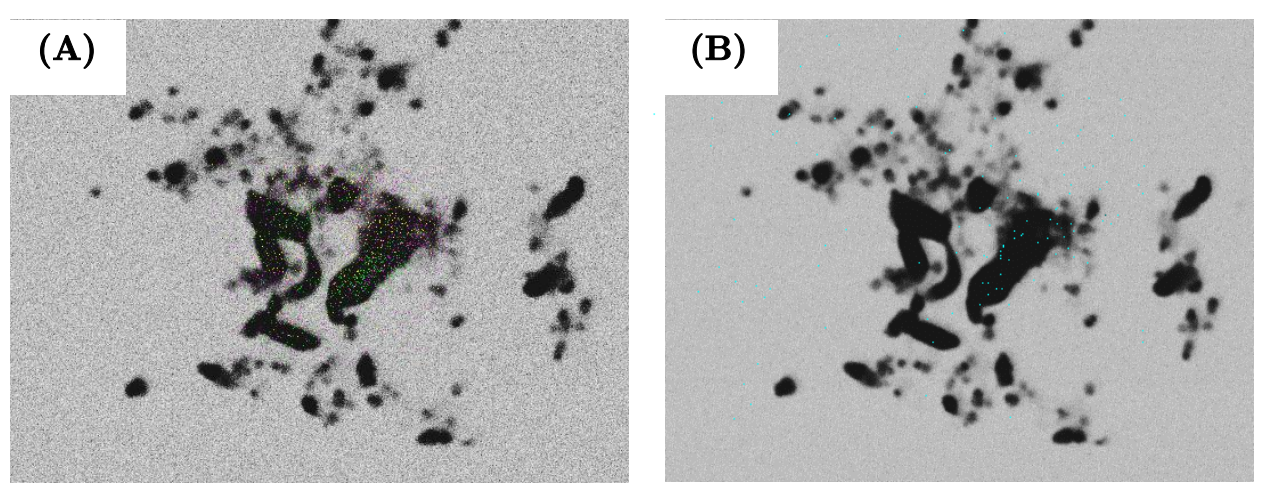


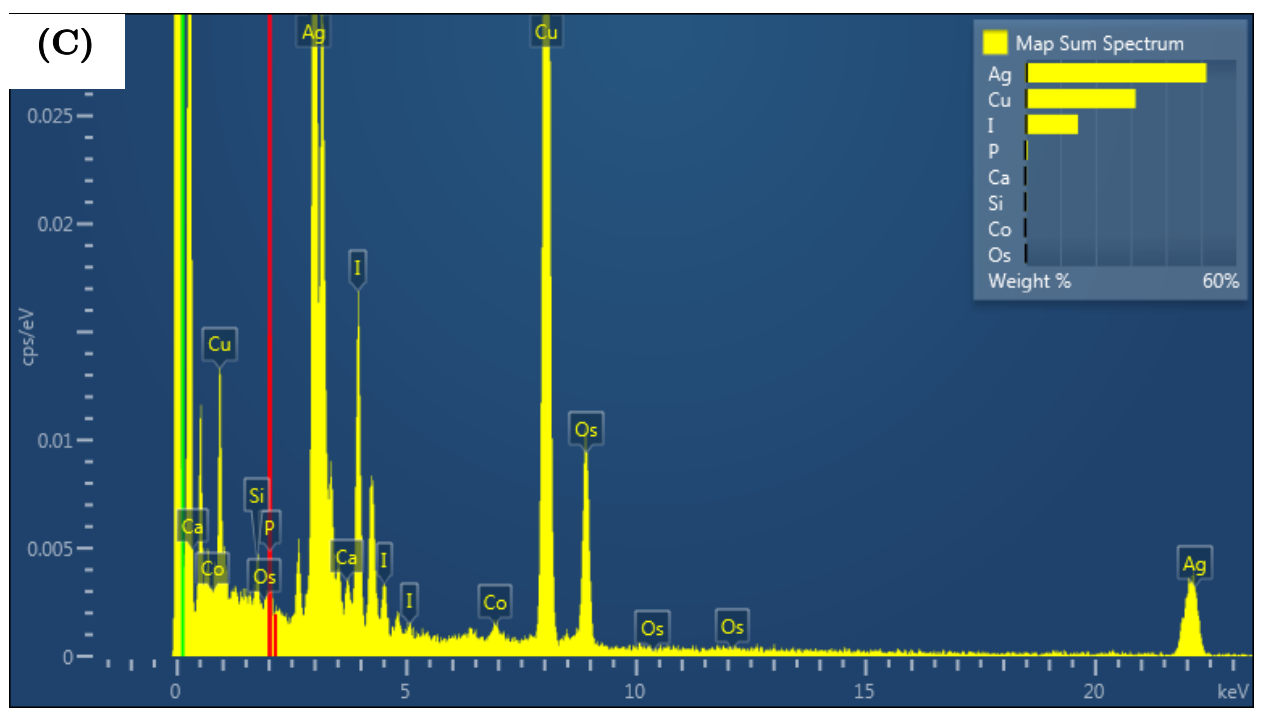

Figure 64. TEM micrographs (low res mode) combined with EDX of $\mathrm{Ag}_{3} \mathrm{PO}_{4}$-AgI post water splitting in $\mathrm{g}-\mathrm{C}_{3} \mathrm{~N}_{4}-\mathrm{I}^{-} / \mathrm{IO}_{3}{ }^{-}-\mathrm{Ag}_{3} \mathrm{PO}_{4}$ system. (A) Full spectrum mapping of TEM-EDX micrograph $(\mathrm{Ag}=$ green, $\mathrm{O}=$ pink, $\mathbf{P}=$ yellow, $\mathbf{I}=$ blue). (B) Iodine mapping of TEM-EDX micrograph. (C) EDX spectrum of corresponding TEM micrograph area $(5 \times 5 \mu \mathrm{m})$, inset denotes weight \% of map sum spectrum. Note: $\mathrm{Cu}$ signal is from the copper/holey carbon grid used as a support, and other heavy elements such as Ca, Si, Co, Os (all 0 wt\%) are artefacts.

6.3.2 Graphitic carbon nitride based Z-scheme water splitting systems

Figure 65(a) shows the XRD data of synthesized $\mathrm{g}-\mathrm{C}_{3} \mathrm{~N}_{4}, \mathrm{BiVO}_{4}$, and $\mathrm{WO}_{3}$. Urea derived $\mathrm{g}-\mathrm{C}_{3} \mathrm{~N}_{4}$ exhibits the usual weak diffraction peaks at $13.0^{\circ}$ (d $=0.681 \mathrm{~nm})$ and $27.4^{\circ}(\mathrm{d}=0.326 \mathrm{~nm})$, corresponding to the approximate dimension of the tri-s-triazine (heptazine) unit, and the distance between graphitic layers respectively ${ }^{151}$. The phase and purity of the synthesised $\mathrm{BiVO}_{4}$ was found to be consistent with the parent literature; pure phase monoclinic point group, space group I2/a ${ }^{17}$. Commercial $\mathrm{WO}_{3}$ was found to be phase identical to photocatalytically active compounds mentioned in the literature; pure phase monoclinic point group, space group P21/n ${ }^{173,242}$. Figure 66 shows the SEM and TEM micrographs of these semiconductors. $\mathrm{WO}_{3}$ particles are large agglomerates (ca. $10 \mu \mathrm{m}$ ), consisting of smaller particles ca. $1 \mu \mathrm{m}$ (Figure 66(A) and (B)). $\mathrm{BiVO}_{4}$ particles range from 100 $\mathrm{nm}$ upwards to $0.5 \mu \mathrm{m}$, in dendritic agglomerates (Figure 66(C)). Graphitic 
carbon nitride is a porous sheet-like compound, with has no fixed particle size (Figure 66(D) and (E)). UV-Vis spectra in Figure 65 (B) shows all materials have visible light absorption, increasing in the order: g- $\mathrm{C}_{3} \mathrm{~N}_{4}$, $\mathrm{WO}_{3}$, and $\mathrm{BiVO}_{4}$; in agreement with the previous reports and signifying the different systems have suitable band gaps for half-reactions ${ }^{178,243}$.
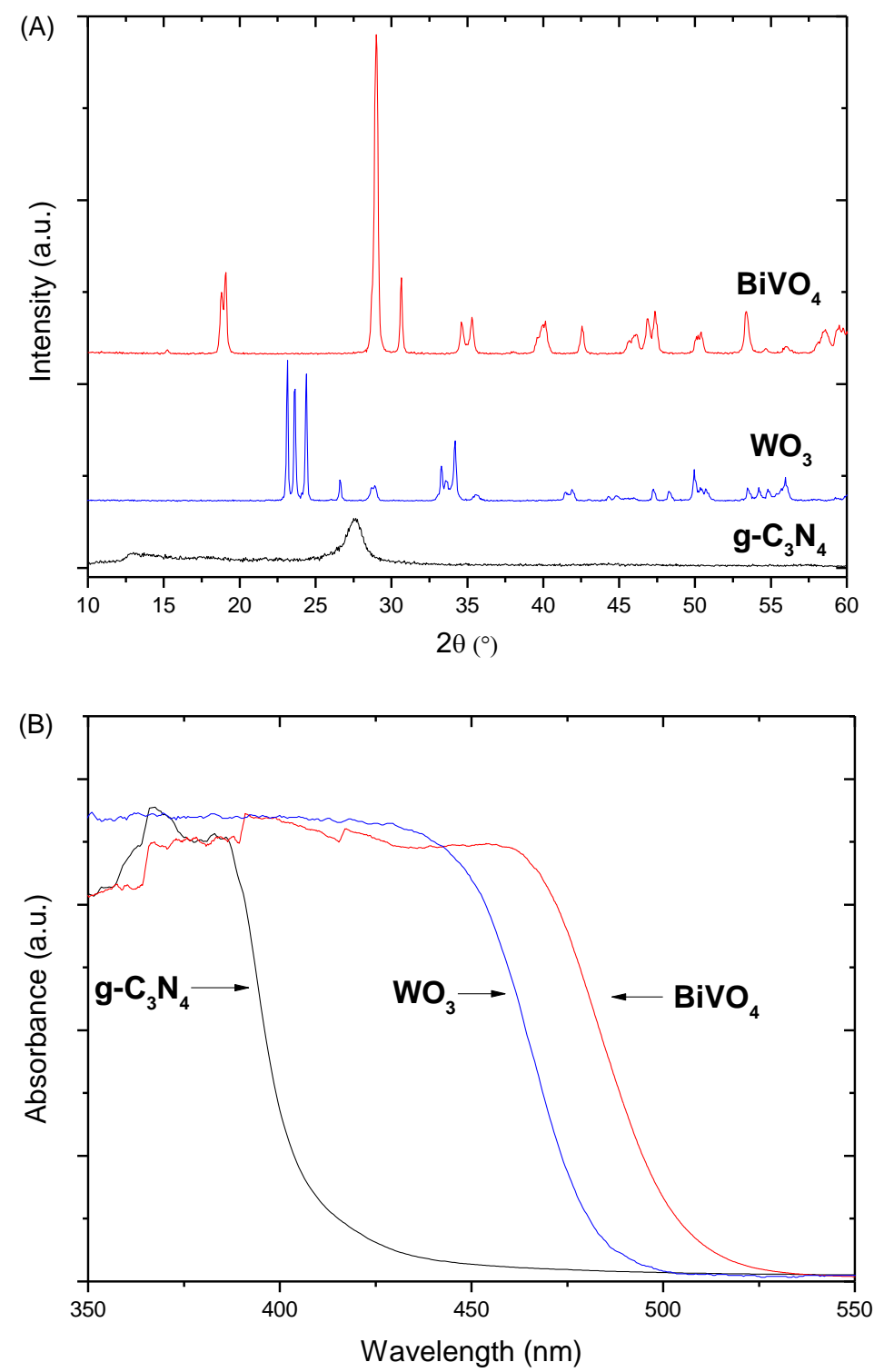

Figure 65. (A) Powder XRD pattern and (B) UV-Vis absorbance spectra of $\mathrm{g}_{-} \mathrm{C}_{3} \mathrm{~N}_{4}, \mathrm{WO}_{3}$ and $\mathrm{BiVO}_{4}$ compounds prior to water splitting reactions 

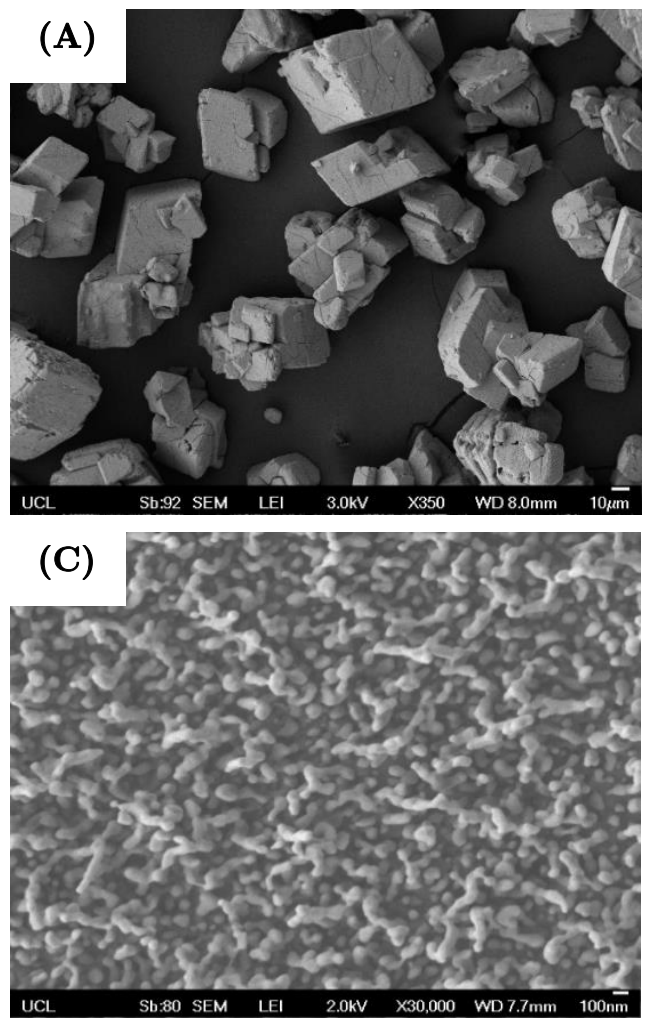

(E)

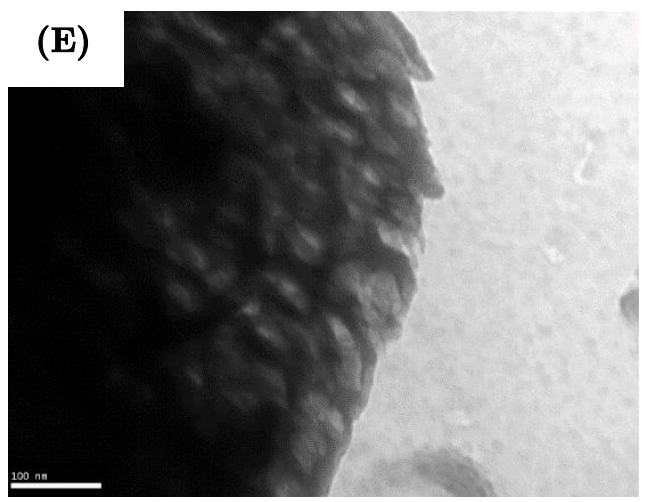



(D)



Figure 66. SEM and TEM micrographs of different photocatalyst. (A) \& (B): Commercial $\mathrm{WO}_{3}$ crystals. (C) Synthesized BiVO ${ }_{4}$ crystals. (D) \& (E) Synthesized g- $\mathrm{C}_{3} \mathrm{~N}_{4}$ sheets.

Initially, Pt-g- $\mathrm{C}_{3} \mathrm{~N}_{4}$ was tested for water splitting in the absence of redox mediators as shown in Table 15(run 1), to which there was no water splitting activity. Pt-loaded $\mathrm{g}-\mathrm{C}_{3} \mathrm{~N}_{4}$ (via photodeposition) is shown to be active for both oxidation of $\mathrm{NaI}\left(\mathrm{I}^{-}\right.$, run 2) and $\mathrm{FeCl}_{2}\left(\mathrm{Fe}^{2+}\right.$, run 3 ) and hydrogen is produced more readily and the relevant chemical reactions were mentioned in section 2.3.3. No oxygen evolution is observed. Then, Pt-g- 
$\mathrm{C}_{3} \mathrm{~N}_{4}$ was tested for water splitting using only redox mediators which can be oxidized. When coupled with a redox mediator, and in combination with either Pt-loaded $\mathrm{WO}_{3}$ (via impregnation, run 4) or $\mathrm{BiVO}_{4}$, pure water splitting is observed.

Table 15. Overall water splitting under full arc irradiation (300 W Xe lamp) using different redox mediated systems

\begin{tabular}{|c|c|c|c|c|c|c|c|}
\hline \multirow[b]{2}{*}{ Run } & \multirow[b]{2}{*}{$\begin{array}{c}\mathbf{H}_{2} \\
\text { photocatalyst } \\
\text { (all } 3 \text { wt\% Pt) }\end{array}$} & \multirow[b]{2}{*}{$\begin{array}{c}\mathrm{O}_{2} \\
\text { photocatalyst }\end{array}$} & \multirow[b]{2}{*}{$\begin{array}{l}\text { weight } \\
\text { ratio } \\
\text { (g:g) }\end{array}$} & \multirow[b]{2}{*}{ pH } & \multirow[b]{2}{*}{$\begin{array}{l}\text { Redox } \\
\text { mediator }\end{array}$} & \multicolumn{2}{|c|}{$\begin{array}{l}\text { Initial gas } \\
\text { evolution } \\
\text { rate }(\mu \mathrm{mol} \mathrm{h} \\
\left.{ }_{1} \mathrm{~g}^{-1}\right)\end{array}$} \\
\hline & & & & & & $\mathbf{H}_{2}$ & $\mathbf{O}_{2}$ \\
\hline 1 & $\mathrm{~g}-\mathrm{C}_{3} \mathrm{~N}_{4}$ & - & - & 7 & - & 0 & - \\
\hline 2 & $\mathrm{~g}-\mathrm{C}_{3} \mathrm{~N}_{4}$ & - & - & 8.3 & $\mathrm{NaI}(5 \mathrm{mM})$ & 5 & - \\
\hline 3 & $\mathrm{~g}-\mathrm{C}_{3} \mathrm{~N}_{4}$ & - & - & 3 & $\underset{\mathrm{mM})}{\mathrm{FeCl}_{2}}(2$ & 4 & - \\
\hline 4 & - & $\mathrm{BiVO}_{4}$ & - & 3 & $\begin{array}{c}\mathrm{FeCl}_{3}(2 \\
\mathrm{mM})\end{array}$ & - & 90 \\
\hline 5 & - & $\mathrm{WO}_{3} \underset{\mathrm{Pt})}{(0.5} \mathrm{wt} \%$ & - & 7 & $\underset{\text { mM) }}{\mathrm{NaIO}_{3}(5}$ & - & 35 \\
\hline 6 & $\mathrm{~g}-\mathrm{C}_{3} \mathrm{~N}_{4}$ & $\mathrm{BiVO}_{4}$ & $1: 1$ & 3 & $\underset{\mathrm{mM})}{\mathrm{FeCl}_{2}}(2$ & 15 & 8 \\
\hline 7 & $\mathrm{~g}-\mathrm{C}_{3} \mathrm{~N}_{4}$ & $\mathrm{BiVO}_{4}$ & $1: 1$ & 3 & $\underset{\mathrm{mM})}{\mathrm{FeCl}_{3}(2}$ & - & 3 \\
\hline 8 & $\mathrm{~g}-\mathrm{C}_{3} \mathrm{~N}_{4}$ & $\mathrm{WO}_{3} \underset{\mathrm{Pt})}{(0.5} \mathrm{wt} \%$ & $1: 1$ & 8.3 & $\mathrm{NaI}(5 \mathrm{mM})$ & 74 & 37 \\
\hline
\end{tabular}

Water splitting over Pt-g- $\mathrm{C}_{3} \mathrm{~N}_{4} / \mathrm{BiVO}_{4}$ in an aqueous $\mathrm{Fe}^{2+/ 3+}$ solution was tested with different starting oxidation states of the redox precursors; $\mathrm{FeCl}_{2}$ (run 6) and $\mathrm{FeCl}_{3}$ (run 7). Starting with $\mathrm{Fe}^{2+}$ species, pure water splitting under full arc commences instantly, and after an initial 1 hour of equilibration, continues steadily. However, if the starting species is $\mathrm{Fe}^{3+}$, the overall water splitting reaction does not occur instantly apart from only $\mathrm{O}_{2}$ evolution. After 15 hours, and re-purging for 2 hours, water splitting occurs due to sufficient $\mathrm{Fe}^{2+}$ existed in the system produced by the previous 15 hour photoreduction process, indicating this side of the reaction proceeds much slower (reduction of $\mathrm{Fe}^{3+}$ by $\mathrm{CB}$ electrons in both $\mathrm{g}_{-} \mathrm{C}_{3} \mathrm{~N}_{4}$ and $\mathrm{BiVO}_{4}$ ). This also indicates that $\mathrm{Fe}^{3+}$ can be regenerated in the present system. As shown in Figure 67 experimental optimization procedures were performed, 
which involved changing $\mathrm{pH}$, photocatalyst amount, and $\mathrm{FeCl}_{2}$ concentration.

It was found that by varying the concentration of $\mathrm{FeCl}_{2}$ in the aqueous solution, water splitting rates can be optimized. At $0.5 \mathrm{mM} \mathrm{FeCl}_{2}$, the water splitting rates are 3.5 and $7.1 \mu \mathrm{mol} \mathrm{h}^{-1} \mathrm{~g}^{-1}$ for oxygen and hydrogen respectively. Increasing the concentration to $2 \mathrm{mM}$ shows that the initial water splitting rate double to 7 and $13.5 \mu \mathrm{mol} \mathrm{h} \mathrm{h}^{-1} \mathrm{~g}^{-1}$ for oxygen and hydrogen respectively. Increasing the concentration to $4 \mathrm{mM} \mathrm{FeCl}_{2}$ then decreases water splitting rates to 4.5 and $9 \mu \mathrm{mol} \mathrm{h}^{-1} \mathrm{~g}^{-1}$ for oxygen and hydrogen respectively. Although no definitive theory has been published to explain this phenomenon ${ }^{181}$, all further optimization procedures were conducted using $2 \mathrm{mM} \mathrm{FeCl} 2$ as it the most efficient concentration for overall water splitting.

The mass ratio between photocatalysts is also crucial to the activity, reaching a maximum at a ratio of $2: 1 \quad\left(\mathrm{~g}-\mathrm{C}_{3} \mathrm{~N}_{4}: \mathrm{BiVO}_{4}\right)$, however, the hydrogen and oxygen evolution rates deviate from stoichiometry, presumably to the larger surface area of $\mathrm{g}-\mathrm{C}_{3} \mathrm{~N}_{4}$ compared to $\mathrm{BiVO}_{4}(43.8$ $\mathrm{m}^{2} \mathrm{~g}^{-1}$ vs. $3.9 \mathrm{~m}^{2} \mathrm{~g}^{-1}$ respectively) and better dispersion in a solution. At 1:1, there is stoichiometry between oxygen and hydrogen, and the rates peak at 7 and $15 \mu \mathrm{mol} \mathrm{h} \mathrm{h}^{-1} \mathrm{~g}^{-1}$ respectively.

Since the $\mathrm{pH}$ of $\mathrm{Fe}^{2+/ 3+}$ systems have previously reported to be sensitive, due to iron ion hydrolysis, we can see an expected optimum at pH 2.4 $3^{243}$. Post water splitting XRD data (Figure 68), however, shows that BiOCl is formed as a sub-phase during the reaction at $\mathrm{pH} 2.4$, whilst at $\mathrm{pH} 3$, $\mathrm{BiVO}_{4}$ did not suffer any degradation. Therefore further testing of the g$\mathrm{C}_{3} \mathrm{~N}_{4}(3 \mathrm{wt} \% \mathrm{Pt})-\mathrm{BiVO}_{4}$ system was conducted at $\mathrm{pH} 3$. 

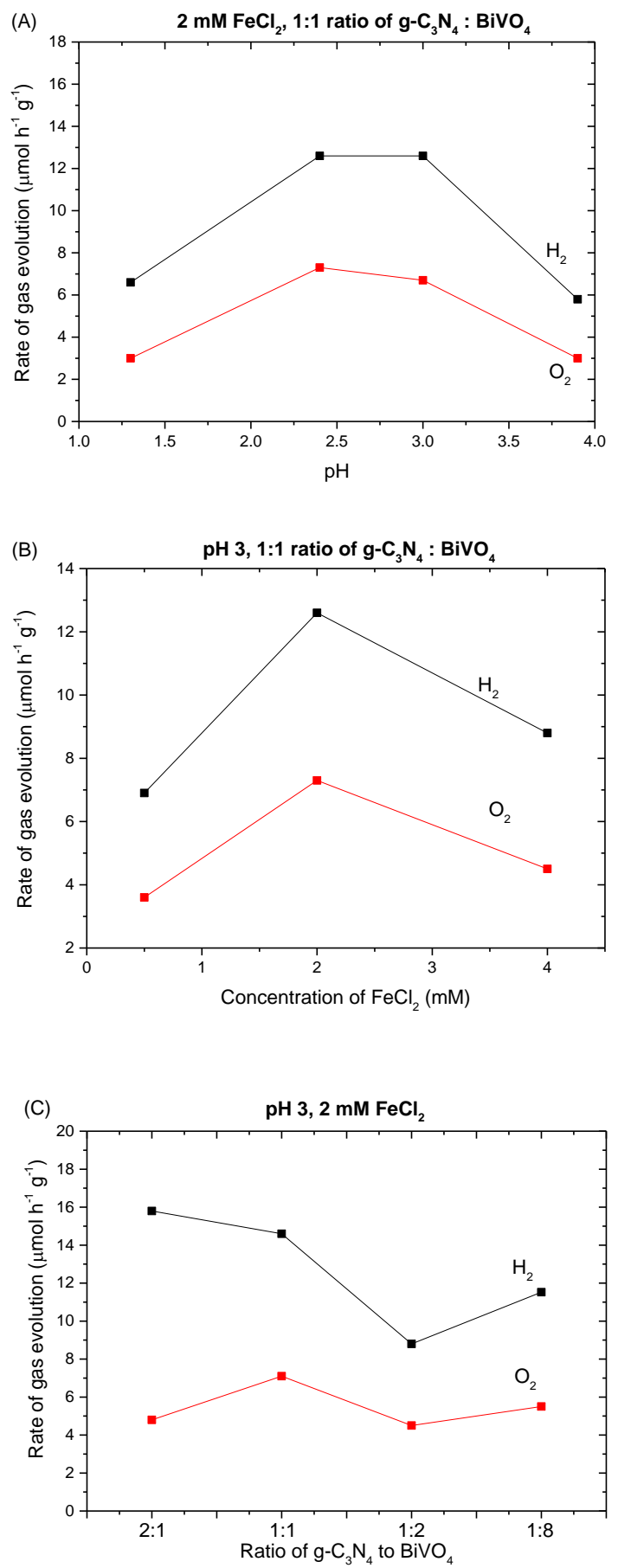

Figure 67. Optimisation of water splitting rates using a g- $\mathrm{C}_{3} \mathrm{~N}_{4}-\mathrm{BiVO}_{4} \mathrm{Z}-$ scheme system with $\mathrm{FeCl}_{2}$ as a redox mediator. (A) $\mathrm{pH}$ modification, (B) differing concentration of $\mathrm{FeCl}_{2}$, (C) alternating the ratio of $\mathrm{g}^{-} \mathrm{C}_{3} \mathrm{~N}_{4}$ to $\mathrm{BiVO}_{4}$. 


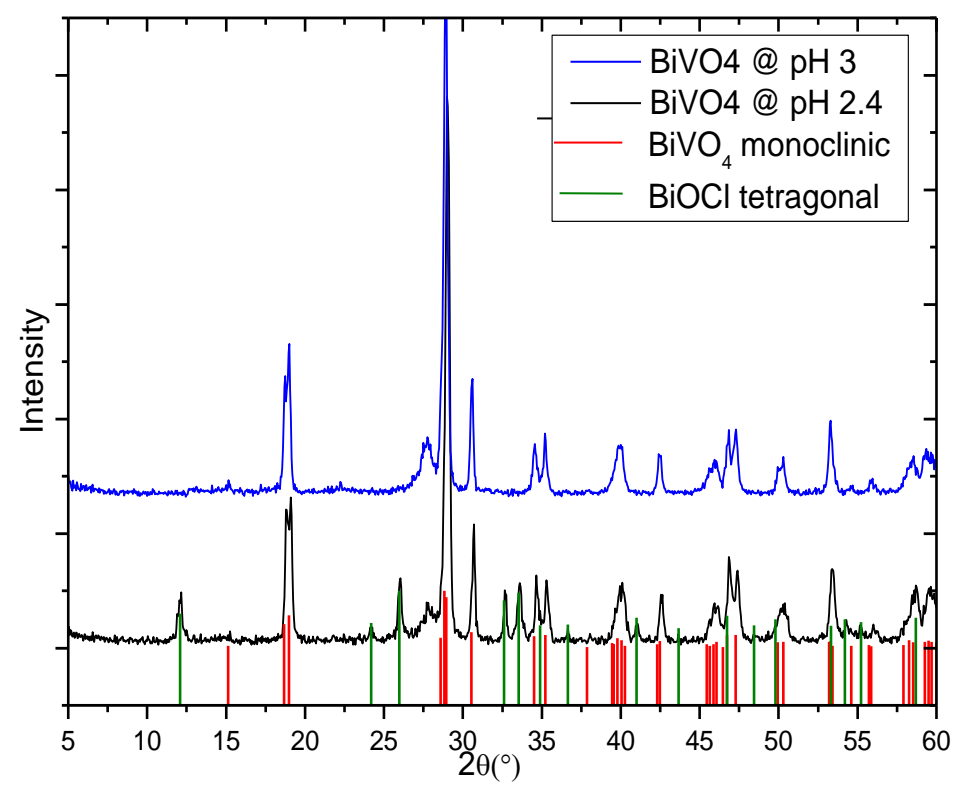

Figure 68. Post water splitting $\mathrm{XRD}$ data of $\mathrm{g}-\mathrm{C}_{3} \mathrm{~N}_{4} / / \mathrm{BiVO}_{4}$ system at different $\mathrm{pH}$ levels. Tetragonal phase BiOCl, JCPDS 00-006-0249, monoclinic phase $\mathrm{BiVO}_{4}$, JCPDS 00-044-0081.

As shown in Figure 69 (A) and (B), under full arc and visible light, both $\mathrm{H}_{2}$ and $\mathrm{O}_{2}$ evolve linearly after an initial 1 hour period of equilibration, for the next 7 hours with a ratio of ca. $2: 1\left(\mathrm{H}_{2}\right.$ and $\mathrm{O}_{2}: 15$ and $8 \mu \mathrm{mol} \mathrm{h} \mathrm{h}^{-1} \mathrm{~g}^{-1}$. Under visible irradiation (a cut-off filter of $395 \mathrm{~nm}$ was used), an average of 6 and $3 \mu \mathrm{mol} \mathrm{h} \mathrm{h}^{-1} \mathrm{~g}^{-1}$ of $\mathrm{H}_{2}$ and $\mathrm{O}_{2}$ has been observed under optimized conditions. The water splitting rate under full arc irradiation is approximately 2.5 times larger than that of the system exposed to visible light $(\lambda>395 \mathrm{~nm})$. However, when considering that the visible light wavelength range of the $\mathrm{Z}$-scheme system is limited by the semiconductor with the largest band gap (i.e. smallest absorption window), g- $\mathrm{C}_{3} \mathrm{~N}_{4}$, the wavelength range is comparatively small; $395<\lambda<415 \mathrm{~nm}$ of the visible light system, and $300<\lambda<415 \mathrm{~nm}$ for the full arc system. Therefore, the visible light system is actually reasonably efficient considering the wavelength range is over 5 times smaller, which affects both energy per photon, and total photon flux. 

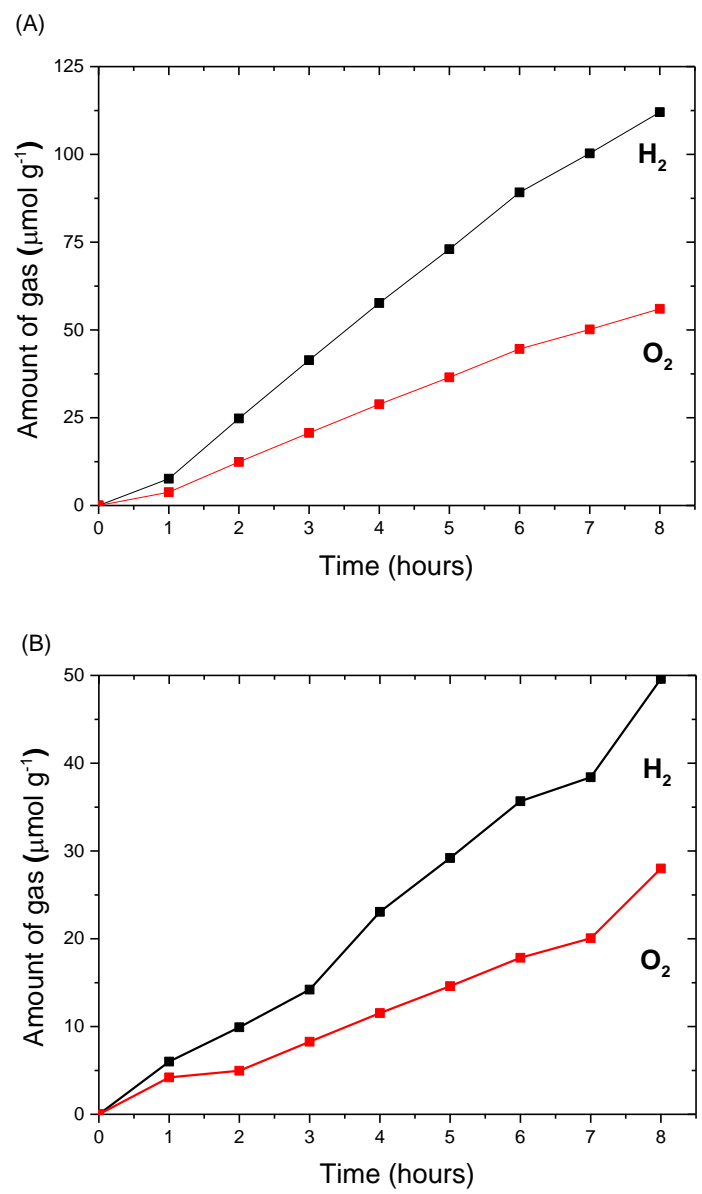

Figure 69. Stoichiometric water splitting (300 W Xe lamp source): (A) g$\mathrm{C}_{3} \mathrm{~N}_{4}(3 \mathrm{wt} \% \mathrm{Pt})-\mathrm{FeCl}_{2}-\mathrm{BiVO}_{4}$ under full arc irradiation, $\mathrm{pH}$ 3, 1:1 photocatalyst weight ratio, $2 \mathrm{mM} \mathrm{FeCl}_{2}$. (B) $\mathrm{g}-\mathrm{C}_{3} \mathrm{~N}_{4}(3 \mathrm{wt} \% \mathrm{Pt})-\mathrm{FeCl}_{2}-$

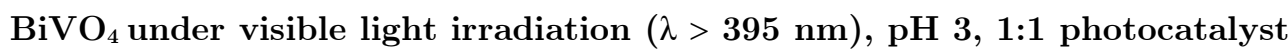
weight ratio, $2 \mathrm{mM} \mathrm{FeCl}_{2}$.

Pt-loaded $\mathrm{g}_{-} \mathrm{C}_{3} \mathrm{~N}_{4}$ was also tested for water splitting when using $\mathrm{NaI}$ as a redox mediator. $\mathrm{Pt}_{-} \mathrm{WO}_{3}(0.5 \% \text { as stated in many reports })^{181}$ was used as a relatively robust photocatalyst $\left(\mathrm{pH}\right.$ dependent) for the reduction of $\mathrm{IO}_{3}$ species produced as a result of $\mathrm{I}^{-}$oxidation by $\mathrm{Pt}-\mathrm{g}-\mathrm{C}_{3} \mathrm{~N}_{4} \cdot{ }^{180}$ Again, the weight ratio of photocatalysts, $\mathrm{pH}$, and mediator concentration were carefully tailored to increase gas evolution rates from water splitting (Figure 70). Whilst $\mathrm{Fe}^{2+/ 3+}$ redox systems are required to operate in acidic conditions, $\mathrm{NaI} / \mathrm{IO}_{3}{ }^{-}$redox systems have been reported to be stable in a $\mathrm{pH}$ range of $7-10$, with $\mathrm{pH} 9$ being optimal for titania based systems ${ }^{178}$. 
However, $\mathrm{WO}_{3}$ is unstable in alkaline media around $\mathrm{pH}$ 9, and forms an oxyanionic tungstate compound or the more likely hydrogentungstate ion ${ }^{244}$ (see Equation 36).

Equation 36

$$
\mathrm{WO}_{3}+\mathrm{OH}^{-} \rightarrow\left[\mathrm{HWO}_{4}\right]^{-}
$$

It is therefore not surprising that the Pt-g- $\mathrm{C}_{3} \mathrm{~N}_{4}-\mathrm{Pt}-\mathrm{WO}_{3}$ system is most efficient at $\mathrm{pH} 8.3$ when using a $\mathrm{NaI} / \mathrm{IO}_{3}{ }^{-}$redox couple (Table 15, run 8). $\mathrm{Pt}-\mathrm{g}-\mathrm{C}_{3} \mathrm{~N}_{4}-\mathrm{Pt}-\mathrm{WO}_{3}$ was not tested in the presence of $\mathrm{Fe}^{2+/ 3+}$ redox simply because $\mathrm{WO}_{3}$ is unstable in acidic conditions $(\mathrm{pH} \leq 3)^{244}$, and undergoes protonation according to:

Equation 37.

$$
W O_{3}+H^{+} \rightarrow H_{y} W O_{x}
$$

The undesirable and ineffective $I_{3}{ }^{-}$anion (Equation 37.), which has shown previously to be detrimental to $\mathrm{I}^{-} / \mathrm{IO}_{3}^{-}$based $\mathrm{Z}$-scheme systems, ceases to be produced in alkaline media ${ }^{178,245}$.

The build-up of the $I_{3}$ ion in acidic media not only negates electron donation from the photocatalyst, but the ion also absorbs light (up to 350 $\mathrm{nm}$ ), hindering photon absorption by the photocatalyst slightly.

Equation 38.

$$
3 I^{-}+2 h^{+} \rightarrow I_{3}^{-}
$$

Alternatively, in neutral and alkaline condition, $\mathrm{I}^{-}$is oxidised to $\mathrm{IO}_{3}{ }^{-}$

Equation 30.

$$
I^{-}+3 \mathrm{H}_{2} \mathrm{O}+6 h^{+} \rightarrow \mathrm{IO}_{3}^{-}+6 \mathrm{H}^{+}(\text {neutral } \mathrm{pH})
$$

Equation 39.

$$
I^{-}+6 \mathrm{OH}^{-}+6 h^{+} \rightarrow \mathrm{IO}_{3}^{-}+3 \mathrm{H}_{2} \mathrm{O}(\text { basic } \mathrm{pH})
$$

Production of $I_{3}$-ions is also hypothesised to be suppressed by the following reaction whereby the photocatalyst is essentially inert ${ }^{178}$ :

Equation 40. 


$$
3 \mathrm{I}_{3}^{-}+6 \mathrm{OH}^{-} \rightarrow 8 \mathrm{I}^{-}+\mathrm{IO}_{3}^{-}+3 \mathrm{H}_{2} \mathrm{O}
$$

Therefore the $\mathrm{pH}$ of this system is particularly important for successful reaction kinetics; $\mathrm{pH} 8.3$ is essentially a point where $\mathrm{WO}_{3}$ is stable, and the production of $\mathrm{I}_{3}{ }^{-}$ions are suppressed, so that water splitting can occur relatively efficiently.
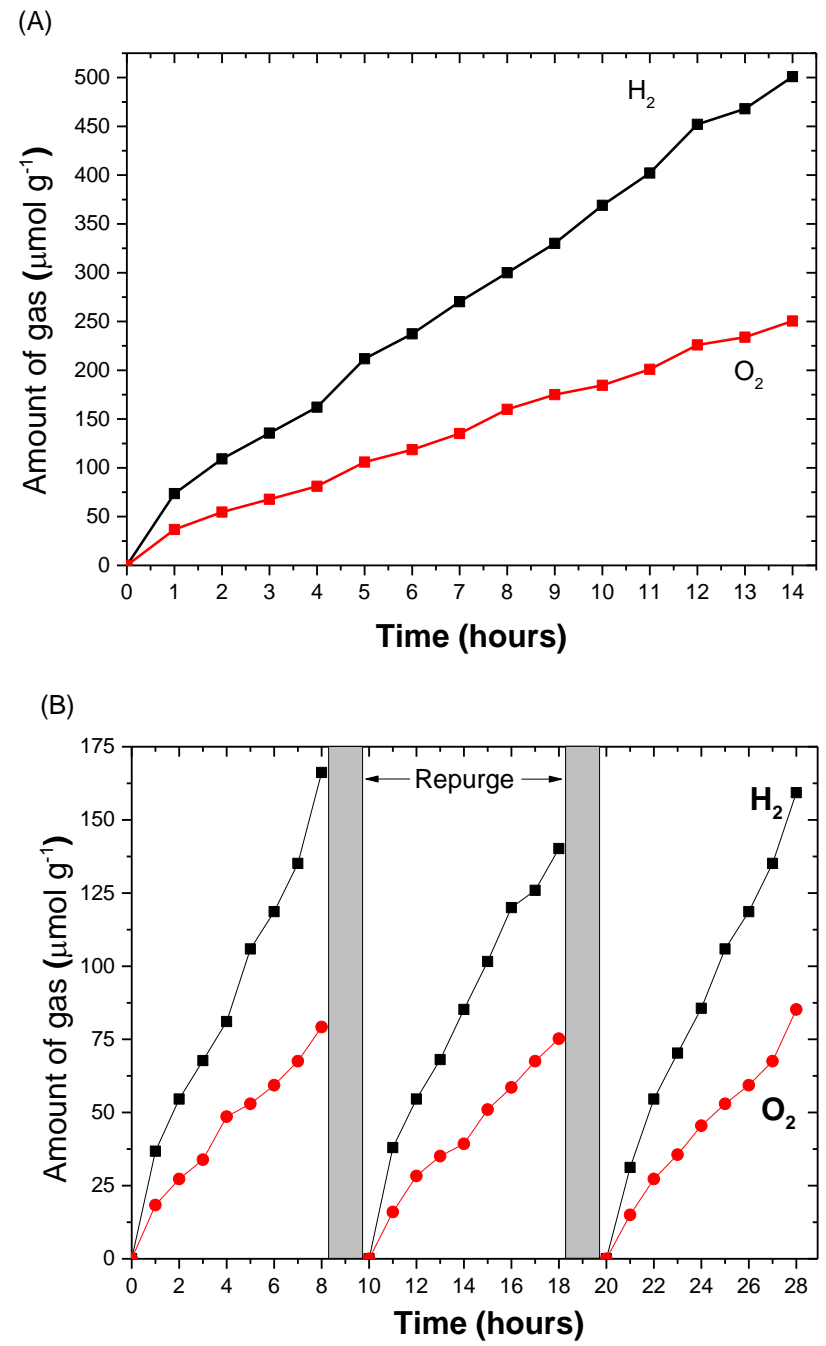

Figure 70. Water splitting using (A) $\mathrm{g}-\mathrm{C}_{3} \mathrm{~N}_{4}(3 \mathrm{wt} \% \mathrm{Pt})-\mathrm{NaI}-\mathrm{WO}_{3}(0.5$ wt\% $\mathrm{Pt}$ ) under full arc irradiation, $\mathrm{pH} 8.3,1: 1$ photocatalyst weight ratio, $5 \mathrm{mM} \mathrm{NaI}$ and, (B) $\mathrm{g}-\mathrm{C}_{3} \mathrm{~N}_{4}(3 \mathrm{wt} \% \mathrm{Pt})-\mathrm{NaI}-\mathrm{WO}_{3}(0.5 \mathrm{wt} \% \mathrm{Pt})$ under visible light irradiation $(\lambda>395 \mathrm{~nm}), \mathrm{pH} 8.3,1: 1$ photocatalyst weight ratio, $5 \mathrm{mM}$ NaI. 
The weight ratio of the two photocatalysts was varied and it was found that similar to the above $\mathrm{g}-\mathrm{C}_{3} \mathrm{~N}_{4}-\mathrm{BiVO}_{4}$ system, this ratio influences the stoichiometry of $\mathrm{H}_{2}$ and $\mathrm{O}_{2}$ production. The optimum weight ratio is 1:1. At 1:1 weight ratio, stable water splitting rates are observed. Averaging over 14 hours, 36 and $18 \mu \mathrm{mol} \mathrm{h}^{-1} \mathrm{~g}^{-1}$ of $\mathrm{H}_{2}$ and $\mathrm{O}_{2}$ are produced, and linearly increase under full arc, shown in Figure 70(A). Under visible irradiation $(\lambda$ $>395 \mathrm{~nm}$ ), an average of 21.2 and $11.0 \mu \mathrm{mol} \mathrm{h} \mathrm{h}^{-1} \mathrm{~g}^{-1}$ of $\mathrm{H}_{2}$ and $\mathrm{O}_{2}$ evolve and the activity is very stable, as indicated by three cycles in Figure 70(B). If the ratio is raised beyond this, water splitting rates decrease, presumably because of an imbalance in light absorption between respective photocatalysts, likely due to a difference in refractive index between the two materials which thus affects the number of photons scattered/reflected. For example, when using $0.3 \mathrm{~g}$ of $\mathrm{Pt}-\mathrm{g}-\mathrm{C}_{3} \mathrm{~N}_{4}$, the hydrogen production rate is initially higher, yet eventually drops off; this is attributed to $\mathrm{Pt}-\mathrm{WO}_{3}$ not absorbing the equivalent photon flux as Pt-g- $\mathrm{C}_{3} \mathrm{~N}_{4}$ (Figure 71). Figure 72 illustrates the unchanged crystal structure of the system post 24 hours water splitting; demonstrating the two organic semiconductor-based systems are extremely stable for pure water splitting.

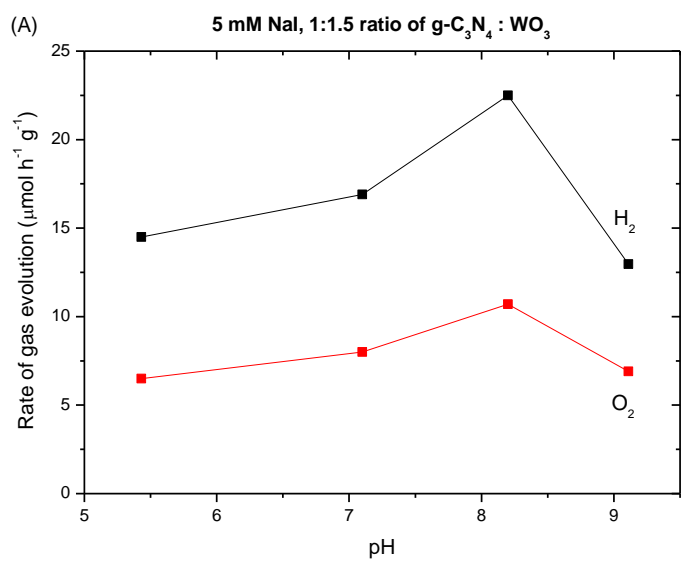



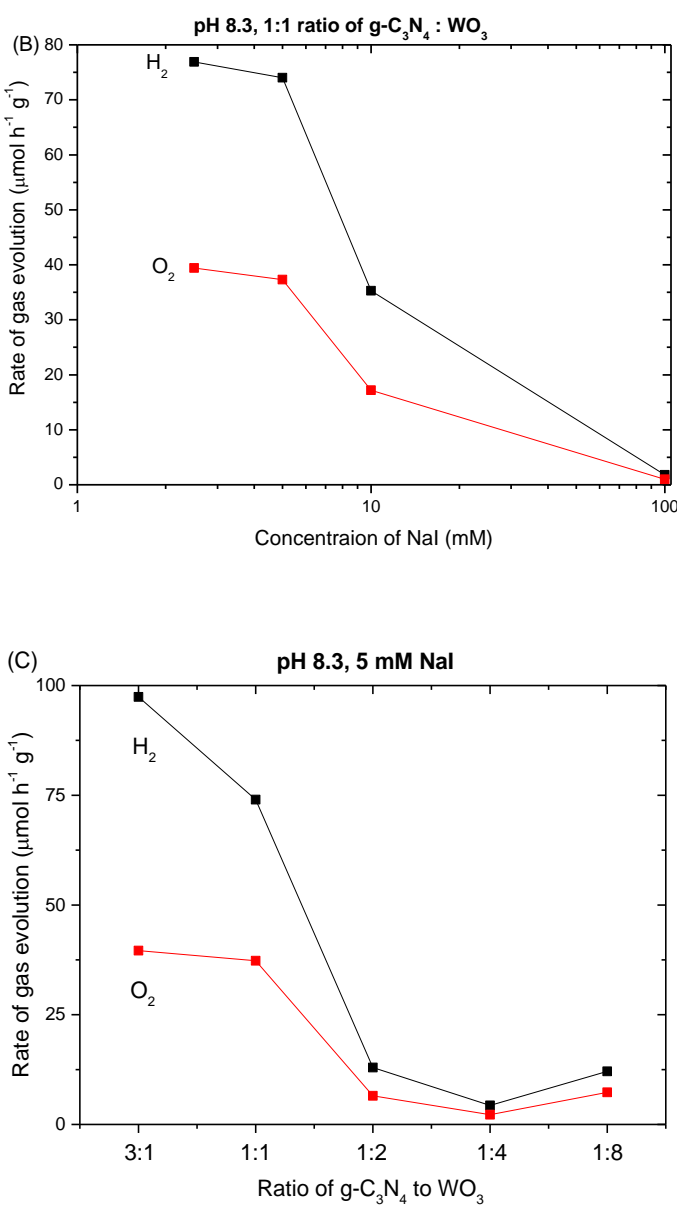

Figure 71. Optimisation of $\mathrm{g}-\mathrm{C}_{3} \mathrm{~N}_{4}-\mathrm{WO}_{3} \mathrm{Z}$-scheme system with $\mathrm{NaI}$ as a redox mediator. (A) $\mathrm{pH}$ modification, (B) differing concentration of NaI, (C) alternating the ratio of $\mathrm{g}-\mathrm{C}_{3} \mathrm{~N}_{4}$ to $\mathrm{WO}_{3}$ 


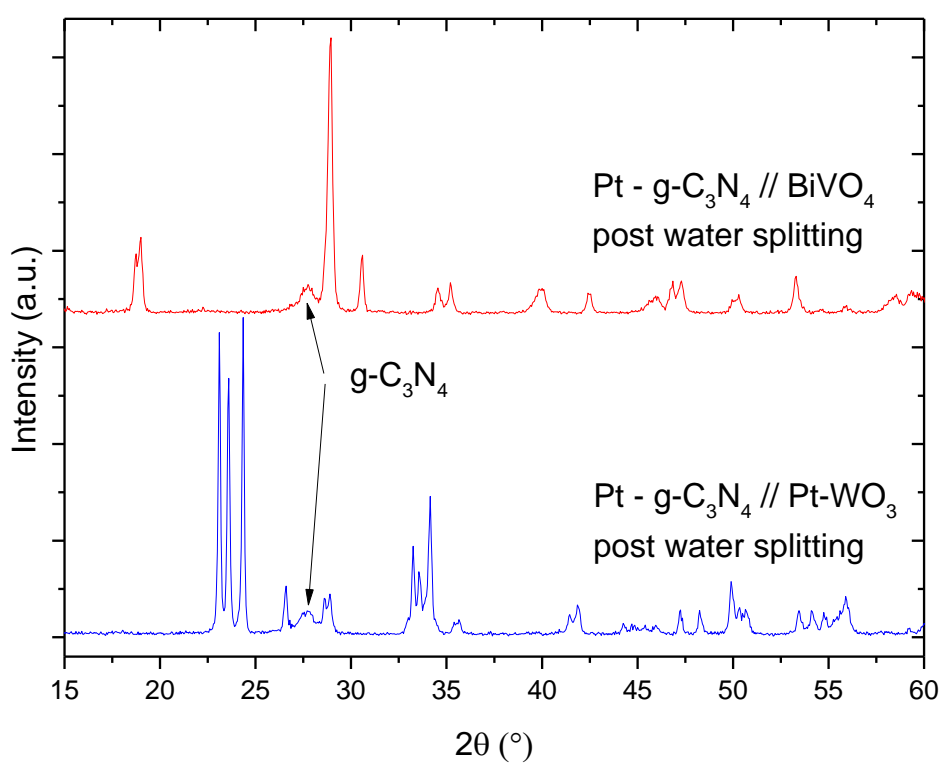

Figure 72. Post XRD diffraction patterns from two different $g-\mathrm{C}_{3} \mathrm{~N}_{4}$ based water splitting systems.

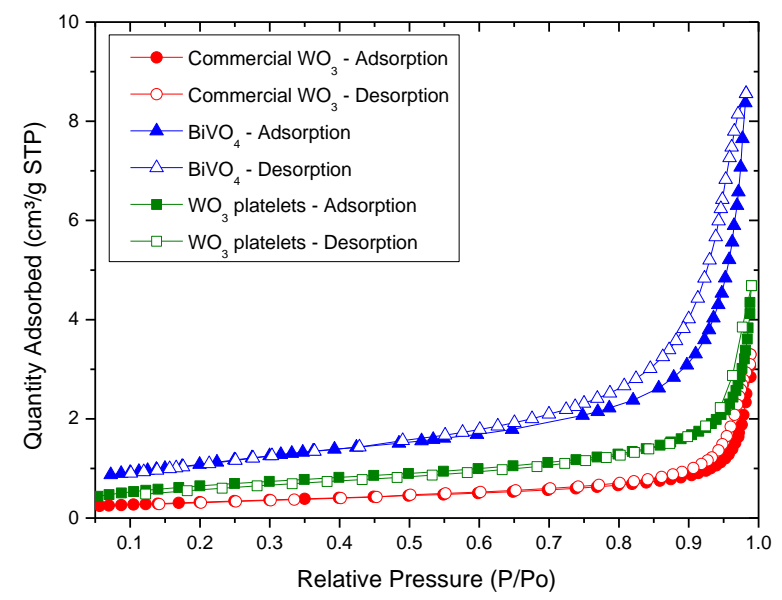

Figure 73. BET isotherms of Commercial $\mathrm{WO}_{3}\left(\mathrm{SSA}=1.15 \mathrm{~m}^{2} \mathrm{~g}^{-1}\right), \mathrm{BiVO}_{4}$ $\left(\mathrm{SSA}=3.91 \mathrm{~m}^{2} \mathrm{~g}^{-1}\right)$, and $\mathrm{WO}_{3}$ platelets $\left(2.45 \mathrm{~m}^{2} \mathrm{~g}^{-1}\right)$.

By far the most efficient system in this study was $\mathrm{g}-\mathrm{C}_{3} \mathrm{~N}_{4}-\mathrm{NaI}-\mathrm{WO}_{3}$, and the solar-to-hydrogen energy conversion efficiency was calculated (STH\%, Equation 10). Using a $150 \mathrm{~W}$ Xe lamp equipped with an AM 1.5 global filter, $100 \mathrm{~mW} \mathrm{~cm}^{-2}$, the current solar energy conversion efficiency is $0.1 \%$.

Despite participating in the most active system for overall water splitting using $\mathrm{g}-\mathrm{C}_{3} \mathrm{~N}_{4}$, it was postulated that the non-optimal morphology and surface area of $\mathrm{WO}_{3}$ (Figure 66 (A) and (B), and Figure 73), could be 
optimised in order to enhance the efficiency. $\mathrm{WO}_{3}$ micro/nanoplates with preferential $\{001\}$ facets were synthesised, phase identical to that of commercial monoclinic $\mathrm{WO}_{3}$ (JCPDS 01-083-0951). As seen in Figure 74, the XRD pattern of the two compounds matches in terms of peak position, indicating the same monoclinic phase. However, upon closer inspection, we see that the intensity ratio of the $(002),(020)$ and (200) peaks at $23.15^{\circ}$, $23.6^{\circ}$ and $24.4^{\circ} 2 \theta$ respectively, vary between samples in comparison to the standard commercial $\mathrm{WO}_{3}$ pattern. The (002) Bragg peak is much more pronounced in comparison to both (020) and (200) peaks on the assynthesised platelet $\mathrm{WO}_{3}$. As shown in Table 16, the intensity ratio of $(002) /(020)$ is 2.50 for platelet $\mathrm{WO}_{3}$, whereas it is only 1.26 for commercial, non-faceted $\mathrm{WO}_{3}$. Similarly the ratio of $(002) /(200)$ for platelet $\mathrm{WO}_{3}$ is 2.20 , and only 1.11 for commercial $\mathrm{WO}_{3}$. This preferential crystal orientation of the (002) plane is evident from SEM micrographs, which demonstrate the plate-like nature (Figure 75). 



Figure 74. Comparison of XRD diffraction patterns of $\mathrm{WO}_{3}$ commercial crystals and as synthesised $\mathrm{WO}_{3}$ platelets. 
Table 16. Intensity ratios of $\mathrm{WO}_{3}$ PXRD Bragg peaks

\begin{tabular}{|c|c|c|c|c|c|}
\hline \multirow[b]{2}{*}{ Sample } & \multicolumn{5}{|c|}{ Peak intensity (20, Reflection plane) } \\
\hline & $21.3^{\circ},(002)$ & $23.6^{\circ},(020)$ & $\begin{array}{c}24.35^{\circ} \\
(200)\end{array}$ & $47.3^{\circ},(004)$ & $48.3^{\circ},(040)$ \\
\hline standard & 3839 & 3779 & 3790 & 417 & 426 \\
\hline $\begin{array}{c}\mathrm{WO}_{3} \\
\text { commercial }\end{array}$ & 1535 & 1223 & 1387 & 133 & 110 \\
\hline $\begin{array}{c}\mathrm{WO}_{3} \\
\text { platelets }\end{array}$ & 2489 & 996 & 1129 & 210 & 91 \\
\hline & $(002) /(002)$ & $(002) /(020)$ & $(002) /(200)$ & $(004) /(004)$ & $(004) /(040)$ \\
\hline $\begin{array}{c}\text { Ratio } \\
\text { Standard }\end{array}$ & 1.00 & 1.02 & 1.01 & 1.00 & 0.98 \\
\hline $\begin{array}{l}\text { Ratio } \mathrm{WO}_{3} \\
\text { commercial }\end{array}$ & 1.00 & 1.26 & 1.11 & 1.00 & 1.21 \\
\hline $\begin{array}{c}\text { Ratio } \mathrm{WO}_{3} \\
\text { platelets }\end{array}$ & 1.00 & 2.50 & 2.20 & 1.00 & 2.31 \\
\hline
\end{tabular}
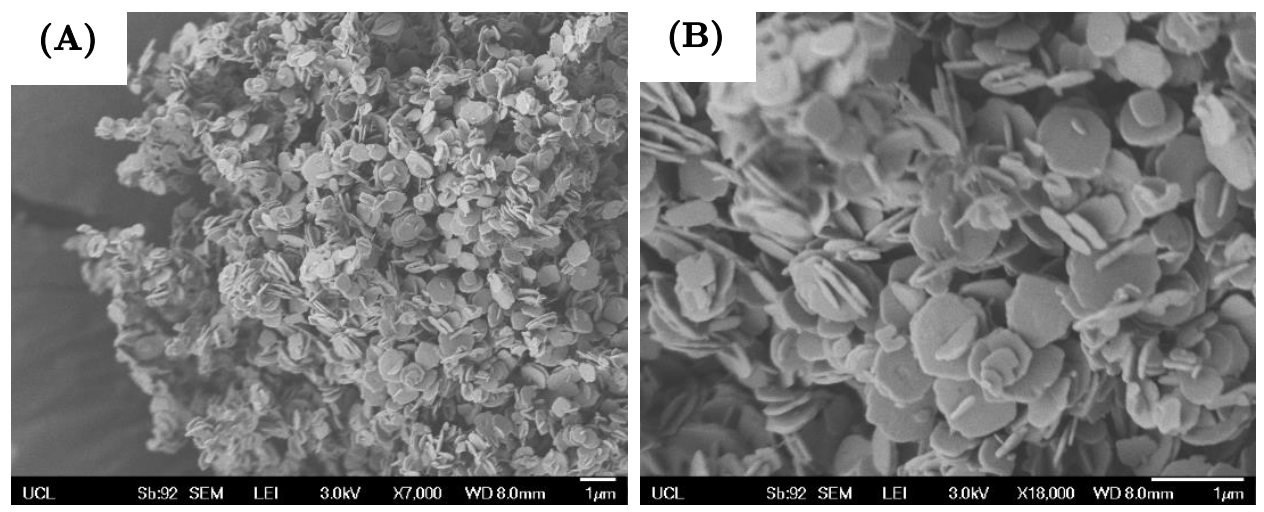

Figure 75. Low (A) and high (B) SEM micrographs of platelet $\mathrm{WO}_{3}$

$0.5 \mathrm{wt} \%$ platinum was deposited as a cocatalyst on the surface, and the platelet $\mathrm{WO}_{3}$ was tested in water splitting system in combination with Ptg- $\mathrm{C}_{3} \mathrm{~N}_{4}$ and $5 \mathrm{mM} \mathrm{NaI}$ acting as a redox mediator. No hydrogen or oxygen was evolved during a 12 hour period; water splitting did not occur. This is surprising, however, a hypothesis could be made when considering evidence from absorption spectra and Tauc plots of the two variants of $\mathrm{WO}_{3}($ Figure 
76). Due to the platelet $\mathrm{WO}_{3}$ particles increased absorption, presumably from the exposing terminated/faceted surface, and smaller indirect band $\operatorname{gap}^{246}(2.48 \mathrm{eV}$ in comparison to $2.62 \mathrm{eV}$, Figure $76(\mathrm{~B})$ and (D)), the conduction band edge could be pushed to more positive potentials by $\sim 0.2$ $\mathrm{eV}$. When considering the energetic requirements needed to reduce $\mathrm{IO}_{3}{ }^{-}$to $\mathrm{I}^{-}$(redox potential of $\mathrm{I}^{-} / \mathrm{IO}_{3^{-}}=+0.67 \mathrm{eV}^{174}$, at $\mathrm{pH}$ 7) and subsequently liberate oxygen in a water splitting system, it is conceivable that this conduction band edge shift (from $\mathrm{CBE}$ of commercial $\mathrm{WO}_{3}+0.6 \mathrm{eV}^{247}$ to that of platelet sample $+0.8 \mathrm{eV}$ at $\mathrm{pH} 7$ ) has negated the ability of $\mathrm{WO}_{3}$ to reduce the redox mediator, $\mathrm{IO}_{3}$, and thus electron hole recombination dominates within the photocatalyst, disabling the production of oxygen from water. The Tauc plot in Figure 76 (C) also show that the $\mathrm{WO}_{3}$ platelets might possess a direct transition of $2.35 \mathrm{eV}$, which might also explain why there is no activity - as the band gap has many sub-band gap states which might act as electron traps and enforce charge recombination.

The change in band gap between the two $\mathrm{WO}_{3}$ samples directly indicates how sensitive the redox mediator-photocatalyst coupling is; a small change in band gap can have a dramatic effect on the effectiveness of the overall water splitting system.

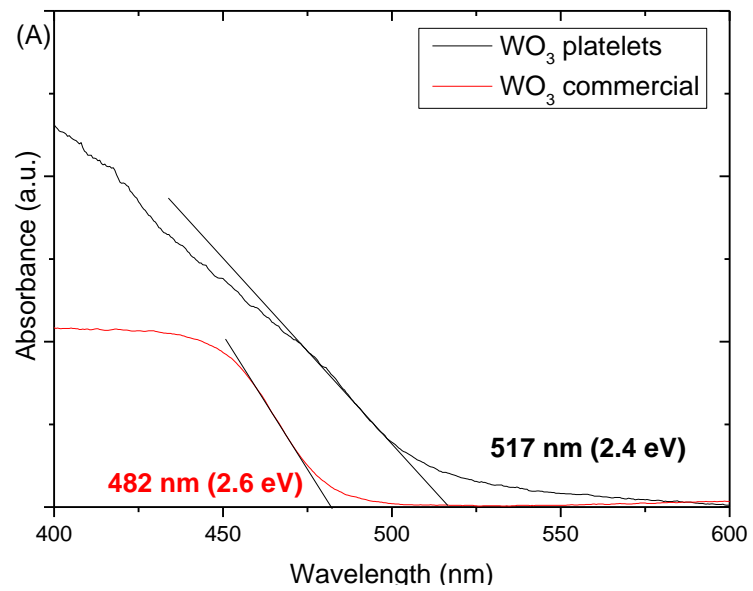



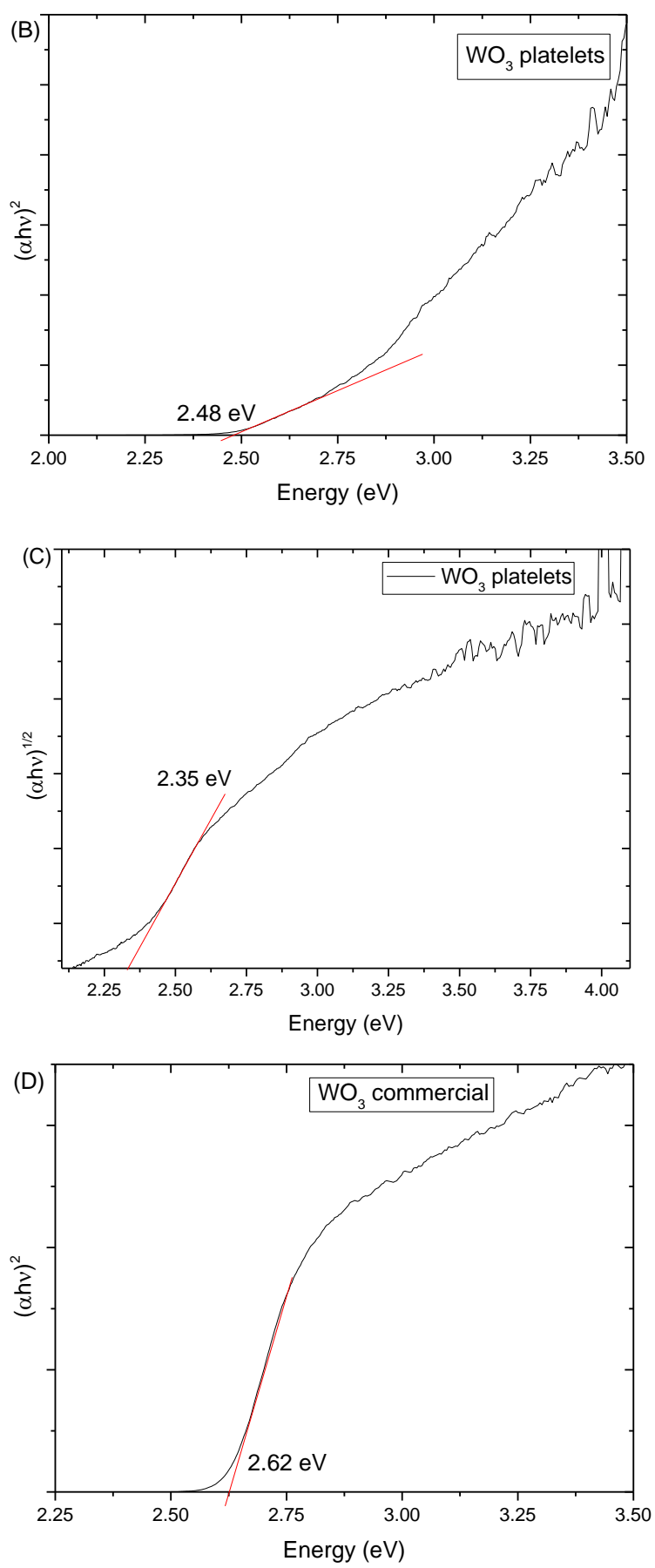


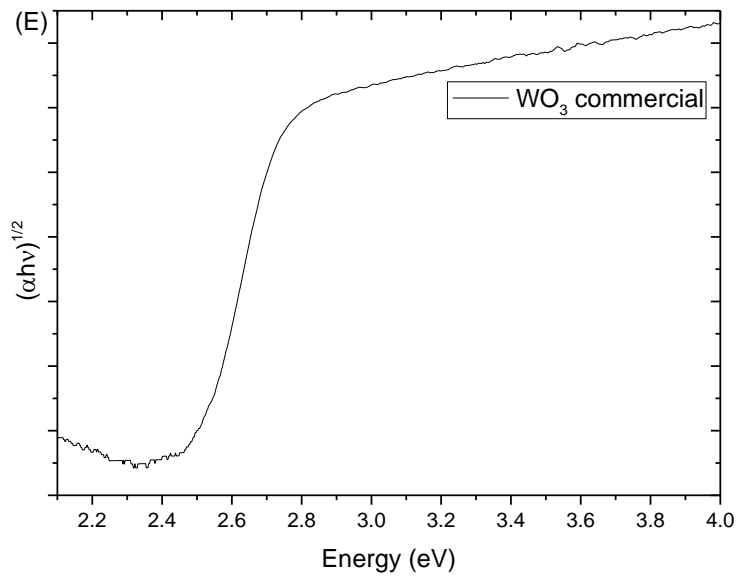

Figure 76. (A) UV-Vis absorption spectra of commercial $\mathrm{WO}_{3}$, and platelet $\mathrm{WO}_{3}$ particles, Tauc plots of; $\mathrm{WO}_{3}$ platelets ( $\mathrm{B} \& \mathrm{C}$ ), and $\mathrm{WO}_{3}$ commercial particles (C \& D). 


\subsection{Conclusions}

To summarise, two novel $\mathrm{g}-\mathrm{C}_{3} \mathrm{~N}_{4}$ based systems for pure water splitting under both UV and visible light irradiation have been demonstrated. The most efficient system is composed of $\mathrm{g}-\mathrm{C}_{3} \mathrm{~N}_{4}$ and $\mathrm{WO}_{3}$ using $\mathrm{I}^{-} / \mathrm{IO}_{3}^{-}$as a soluble redox mediator, which yields 36 and $18 \mu \mathrm{mol} \mathrm{h}^{-1} \mathrm{~g}^{-1}$ of hydrogen and oxygen respectively. The stability of the system is evidenced in the linear gas evolution over 28 hours. This leads to a $\mathrm{STH} \%$ of ca. $0.1 \%$. Both $\mathrm{BiVO}_{4}$ $\left(\mathrm{Fe}^{2+/ 3+}\right)$ and $\mathrm{WO}_{3}\left(\mathrm{I} / \mathrm{IO}_{3}{ }^{-}\right)$based systems were shown to be heavily dependent on $\mathrm{pH}$, weight ratio between photocatalysts, and redox mediator concentration. The energetic sensitivity of the $\mathrm{WO}_{3}\left(\mathrm{I} / \mathrm{IO}_{3}^{-}\right)$system was demonstrated by testing $\mathrm{WO}_{3}$ platelets, which have a smaller band gap than their commercial counterparts, and this was shown to have a dramatic negative effect upon the system - no water splitting was observed. However, the findings pave the way for more systems to be created that are based on urea-derived $\mathrm{g}-\mathrm{C}_{3} \mathrm{~N}_{4}$, provided the band positioning of the semiconductor is carefully controlled.

Carbon nitride synthesized using other precursors such as dicyandiamide or thiourea was also tested for water splitting using both $\mathrm{NaI}-\mathrm{WO}_{3}$ and $\mathrm{FeCl}_{2}-\mathrm{BiVO}_{4}$ systems, yet do not give detectable activity, conceivably due to the low efficiency of these carbon nitrides in comparison to urea-derived g- $\mathrm{C}_{3} \mathrm{~N}_{4}$. Low efficiency as a result of a smaller driving force/band gap could lead to an imbalance in redox mediation species, or become comparative to competitive side reactions ( $\mathrm{I}^{-}$oxidation to $\mathrm{I}_{3}{ }^{-}$instead of $\mathrm{IO}_{3}{ }^{-}$) which would eventually halt the water splitting reaction. It is well documented that redox mediator ions such as $\mathrm{I} / \mathrm{IO}_{3}{ }^{-}$and $\mathrm{Fe}^{2+/ 3+}$ are particularly sensitive when adsorbing to surfaces, as explored by Abe et al, and Ohno et al ${ }^{178,248}$. The studies show that the $\mathrm{IO}_{3}^{-}$ion preferentially adsorbs on to a rutile surface rather than anatase, and is effectively reduced, meanwhile water is effectively oxidised by rutile $\mathrm{TiO}_{2}$. Considering anatase and rutile have different isoelectric points(anatase ca. 5 , rutile $<3)^{249}$, and therefore surface charge, it is possible the reason behind the selective absorption is due to zeta potential at the surface. The same premise could apply with g- $\mathrm{C}_{3} \mathrm{~N}_{4}$, since the degree of protonation or surface acidity varies from sample to sample. In which case, the conjecture would be that $\mathrm{I}^{-}$ions selectively bind to urea based $\mathrm{g}-\mathrm{C}_{3} \mathrm{~N}_{4}$ due to the relatively small proton concentration, and it is more difficult to bind to DCDA or thiourea based carbon nitride as the potential is larger. 
It was found that $\mathrm{Ag}_{3} \mathrm{PO}_{4}$ was not able to participate in a $\mathrm{Z}$-scheme system whereby $\mathrm{FeCl}_{3}$ or $\mathrm{NaI}$ are the redox precursors, due to interaction with the mediator, and unsuitable $\mathrm{pH}$ ranges. 


\section{Overall conclusions}

A wide variety of literature was studied in chapter 2 in order to identify the benchmark materials developed and more importantly the barrier which limits the solar to $\mathbf{H}_{2}$ evolution efficiency. Novel strategies were then attempted in order to tackle the difficulties experienced in the field.

The first publication on $\mathrm{Ag}_{3} \mathrm{PO}_{4}$ by $\mathrm{Yi}$ et al displayed great promise, demonstrating a relatively good quantum yield for a novel photocatalyst, but the efficiency was not high enough, in particular at long wavelength (e.g. $500 \mathrm{~nm}$ ) for the photooxidation of water ${ }^{7}$. It was unclear as to what would further augment or diminish the activity, and thus was selected to be studied for water photooxidation. In parallel, graphitic carbon nitride was also thought to be promising as a visible light hydrogen production photocatalyst. Despite the relatively low quantum yield reported in the first instance, the compound is very stable chemically, and also is incredibly cheap to synthesise. Considering these arguments, it was decided that the understanding and modification of $\mathrm{g}-\mathrm{C}_{3} \mathrm{~N}_{4}$ would be pursued. Z-scheme photocatalytic systems have been reported, yet for only a relatively small number of photocatalysts. It was later postulated that either $\mathrm{g}-\mathrm{C}_{3} \mathrm{~N}_{4}$ or $\mathrm{Ag}_{3} \mathrm{PO}_{4}$ could potentially be integrated into a $\mathrm{Z}$-scheme system, based on their band structure and good photocatalytic activity.

Firstly the development of a reliable water splitting test station was described; a combination of both a custom designed, gas tight, borosilicate reactor, and the integration of this with a gas chromatograph. The gas chromatography unit was calibrated to accurately determine unknown quantities of hydrogen and oxygen, down to the nanomole scale, and with a small sampling error of $0.52 \%$ and $0.97 \%$ for hydrogen and oxygen respectively.

Then the focus of the thesis is directed towards the development of efficient water photooxidation using a $\mathrm{Ag}_{3} \mathrm{PO}_{4}$ photocatalyst. A method was found whereby to roughly spherical silver phosphate exhibited a photooxidative ability analogous to that published by $\mathrm{Yi}$ et al, using ethanol, $\mathrm{AgNO}_{3}$, and $\mathrm{Na}_{2} \mathrm{HPO}_{4}$. Upon experimentation with phosphate precursors, it was found that $\mathrm{H}_{3} \mathrm{PO}_{4}$ and the relative concentration could slow the growth of $\mathrm{Ag}_{3} \mathrm{PO}_{4}$ crystals, and retain low index facets, resulting in the production of novel $\{111\}$ terminated tetrahedral $\mathrm{Ag}_{3} \mathrm{PO}_{4}$ crystals. $\mathrm{By}$ changing the concentration of $\mathrm{H}_{3} \mathrm{PO}_{4}$, and thus limiting the kinetics of growth, it is possible to control the growth of $\mathrm{Ag}_{3} \mathrm{PO}_{4}$ crystals from tetrapods to tetrahedrons. $\mathrm{Ag}_{3} \mathrm{PO}_{4}$ tetrahedrons demonstrate considerably higher 
oxygen evolution activity in comparison to roughly spherical particles, and other low index facets such as $\{100\}$ and $\{100\}$. DFT calculations were used to model the low index surfaces' surface energy, and the hole mass of $\mathrm{Ag}_{3} \mathrm{PO}_{4}$ in different directions. It is concluded that a combination of highest surface energy and lowest hole mass on $\mathrm{Ag}_{3} \mathrm{PO}_{4}\{111\}$ surfaces results in an extremely high oxygen evolution from water, with an internal quantum yield of nearly unity at $400 \mathrm{~nm}$ and $80 \%$ at $500 \mathrm{~nm}$.

The thesis then investigates $\mathrm{g}-\mathrm{C}_{3} \mathrm{~N}_{4}$ as a hydrogen producing photocatalyst. Graphitic carbon nitride was synthesised via thermal decomposition, using 4 different precursors, at various calcination temperatures, calcination ramp rates, and varying cocatalyst element and weighting. A new synthesis method for the production of urea derived graphitic carbon nitride was developed. The most active sample was synthesised at $600{ }^{\circ} \mathrm{C}$, at $5^{\circ} \mathrm{C} /$ minute, and by using $3 \mathrm{wt} \%$ platinum as a cocatalyst. This sample evolved hydrogen from water in the presence of TEOA at $3327.5 \mu \mathrm{mol} \mathrm{hr}{ }^{-1}$ $\mathrm{g}^{-1}$ under visible light $(\lambda>395 \mathrm{~nm})$, and ca. $20,000 \mu \mathrm{mol} \mathrm{g}^{-1}$ under full arc irradiation. The internal quantum yield in the visible region is $26.5 \%$, nearly ten times larger than previous literature examples, or the sample synthesised using MCA/DMSO. In order to determine the reason for the high reactivity, in comparison to other examples, a series of characterisation methods were employed. TGA-DSC-MS was used to monitor the formation mechanism of $\mathrm{g}-\mathrm{C}_{3} \mathrm{~N}_{4}$ derived from both urea and thiourea - which was found to be drastically different during the primordial stages of synthesis in comparison to cyanamide ${ }^{151}$ and thiourea. In the case of urea derived g$\mathrm{C}_{3} \mathrm{~N}_{4}$, it is shown that the high surface area and porosity is created by the continuous loss of $\mathrm{CO}_{2}$, a result not seen before. Formaldehyde $\left(\mathrm{CH}_{2} \mathrm{O}\right)$ is also continually produced throughout the decomposition of urea to g- $\mathrm{C}_{3} \mathrm{~N}_{4}$, a process which is proposed to reduce the percentage of hydrogen/protons in carbon nitride. Only trace amounts of $\mathrm{CS}_{2}$ and $\mathrm{CH}_{4} \mathrm{~N}_{2}$ remain at the point of polymerisation $\left(500{ }^{\circ} \mathrm{C}\right)$ in the thermal condensation of thiourea derived g- $\mathrm{C}_{3} \mathrm{~N}_{4}$, therefore at the point of polymerisation, the compound is not losing extra hydrogen/protons from fragmented products.

Using the N1s core level data obtained from XPS, it is shown that across all synthesised samples, there is an inversely proportional relationship between hydrogen/proton content in the photocatalyst and hydrogen production rate achieved by the photocatalyst. A larger hydrogen/proton content leads to a lower hydrogen evolution rate. This is also confirmed by Elemental Analysis, which although is not specific as to where the hydrogen 
is located, the trend is the same. It has been shown in previous studies that a less polymeric carbon nitride intrinsically has more hydrogen/protons due to breakages. The trend in XRD data shows that a greater level of condensation, and thus a less polymerised $\mathrm{g}-\mathrm{C}_{3} \mathrm{~N}_{4}$, leads to a less active photocatalyst.

DFT calculations were used to show that increasing the hydrogen/proton content causes a positive shift in the conduction band edge (with respect to NHE), therefore reducing the driving force for reduction reactions. Furthermore, TDDFT calculations demonstrate that excess protonation causes excited photoelectrons to become localised at non-active redox sites, which is further detrimental to the photocatalytic ability since these charge carriers cannot participate in water splitting reactions.

Due to the progress made in the previous two chapters, the feasibility of the integration of either $\mathrm{Ag}_{3} \mathrm{PO}_{4}$ or $\mathrm{g}_{-} \mathrm{C}_{3} \mathrm{~N}_{4}$ into a $\mathrm{Z}$-Scheme for water splitting was then assessed. $\mathrm{Ag}_{3} \mathrm{PO}_{4}$ was found to not function as an oxygen evolving photocatalyst in Z-scheme water splitting systems due to the incompatibility between photocatalyst and synthetic conditions. $\mathrm{Ag}_{3} \mathrm{PO}_{4}$ dissolves into the solution at the $\mathrm{pH}$ range $(\leq 3.5)$ required for $\mathrm{Fe}^{2+/ 3+}$ to be an effective redox mediator, and also reacts with $\mathrm{H}_{2} \mathrm{SO}_{4}$ at $\mathrm{pH} 4$ to form an $\mathrm{Ag}_{2} \mathrm{SO}_{4}-\mathrm{Ag}_{3} \mathrm{PO}_{4}$ mixed phase compound (evidenced by XRD) which shows no activity for water splitting. When $\mathrm{NaI}$ is used, $\mathrm{Ag}_{3} \mathrm{PO}_{4}$ reacts with the iodide anion to form AgI as seen in TEM and EDX, visibly changing the colour of the solution and also demonstrating no activity for overall water splitting.

Urea derived $\mathrm{g}-\mathrm{C}_{3} \mathrm{~N}_{4}$ is shown to actively participate in novel Z-Scheme water splitting systems as a hydrogen evolving photocatalyst. Due to the robustness and favourable band position, $\mathrm{g}-\mathrm{C}_{3} \mathrm{~N}_{4}$ can be coupled with either $\mathrm{I} / \mathrm{IO}_{3}{ }^{-}$or $\mathrm{Fe}^{2+/ 3+}$ redox mediators, at any $\mathrm{pH}$, and with Pt-loaded $\mathrm{WO}_{3}$ or $\mathrm{BiVO}_{4}$ respectively. The highest water splitting rates were achieved using a g- $\mathrm{C}_{3} \mathrm{~N}_{4}-\mathrm{NaI}-\mathrm{WO}_{3}$ system, peaking at 36 and $18 \mu \mathrm{mol} \mathrm{h}^{-1} \mathrm{~g}^{-1}$ of hydrogen and oxygen respectively. The largest $\mathrm{STH} \%$ was recorded at $0.1 \%$ under AM 1.5 illumination. This is far from the commercial target of $10 \%$ for overall water splitting systems, yet for a novel system, can be built on and improved. Carbon nitride fabricated by thermal decomposition of dicyandiamide or thiourea do not give detectable activity for any Z-scheme water splitting reaction, possibly due to the low efficiency of these carbon nitrides in comparison to urea-derived $\mathrm{g}-\mathrm{C}_{3} \mathrm{~N}_{4}{ }^{227}$. Low efficiency as a result of a smaller driving force/higher degree of protonation could lead to an 
imbalance in redox mediation species, or become comparative to competitive side reactions ( $\mathrm{I}^{-}$oxidation to $\mathrm{I}_{3}{ }^{-}$instead of $\mathrm{IO}_{3}{ }^{-}$) which would eventually halt the water splitting reaction. It may also be possible that $\mathbf{I}^{-}$ ions could selectively bind to urea based $\mathrm{g}-\mathrm{C}_{3} \mathrm{~N}_{4}$ due to the relatively small proton concentration, and it is more difficult to bind to DCDA or thiourea based carbon nitride as the surface zeta potential is larger. 


\section{Future Work}

The thesis achieved the main goals set out, by adding novel scientific understanding to the nature of the activity demonstrated by some of the newest photocatalysts. Despite these successes, and taking into account the originality of the photocatalysts investigated, the research should also be viewed as a platform to be built upon, and therefore there are many possible follow up investigations that could be pursued in order to meet the $10 \%$ solar to $\mathrm{H}_{2}$ conversion efficiency using a robust system.

Chapter 4 detailed a facile method to control the exposing facet of $\mathrm{Ag}_{3} \mathrm{PO}_{4}$ and the influence on the activity. For any industrial application, $\mathrm{Ag}^{+}$cannot be used as an electron scavenger as metallic silver eventually poisons the surface, it is used here as a method to demonstrate a proof-of-concept in terms of potential maximum efficiency. Therefore, a sizable project would be to attempt to grow $\mathrm{Ag}_{3} \mathrm{PO}_{4}$ tetrahedrons onto a thin film, for use in a PEC cell. The contact between conducting substrate and photocatalyst would have to be exceptionally good to shuttle electrons around a circuit, otherwise as shown by $\mathrm{Yi}$ et al, in the absence of a suitable voltage, $\mathrm{Ag}_{3} \mathrm{PO}_{4}$ undergoes photocorrosion. Similarly, photocorrosion occurs over $\mathrm{Ag}_{3} \mathrm{PO}_{4}$ in the absence of soluble electron scavengers. It might also be possible to cover $\mathrm{Ag}_{3} \mathrm{PO}_{4}$ in a stable protection layer which is transparent to visible light, in order to act as an electron scavenger and prevent photocorrosion. Possibilities for cutting edge protection layers include; iron ferrihydrite (very high electronegativity), $\mathrm{TiO}_{2}$ (robust over-layer), $\mathrm{ZnO} \mathrm{Al}$ or silica $^{250,251,252}$.

In chapter 5, it was shown that by controlling the protonation status of g$\mathrm{C}_{3} \mathrm{~N}_{4}$, it is possible to increase the quantum efficiency up to $26 \%$ under visible light. Previous studies have shown surface area to have an impact of sorts on the HER, however in comparison to altering the proton content, the increase in IQY is minimal. Investigations by Transient Absorption Spectroscopy (TAS) have previously provided understanding as to rate limiting steps in other photocatalysts such as $\mathrm{TiO}_{2}$ and $\mathrm{Fe}_{2} \mathrm{O}_{3}{ }^{30,42,215}$. This same technique could be applied to carbon nitride to examine the kinetics of photogenerated charge carriers as a function of incident wavelength, which would illustrate a way to further improve the efficiency for the photocatalyst, e.g. by tailoring porosity and surface acidity.

Z-Scheme water splitting systems were studied in chapter 6 , and $\mathrm{g}^{-} \mathrm{C}_{3} \mathrm{~N}_{4}$ was shown to be a functional photocatalyst for both oxidation of $\mathrm{Fe}^{2+}$ or $\mathrm{I}$ . Despite the high quantum efficiency demonstrated in chapter 5 , in 
sacrificial systems, the STH\% of the best Z-scheme system was low, at $0.1 \%$. Therefore, a further study would be required to probe this novel system. One route would be to experiment with alternative redox mediators, yet this would prove difficult as there are only a handful of mediators which are suitable for Z-Scheme water splitting. As mentioned, it is unknown whether the absorption of iodide or ferrous ions is surface-selective. Hence, another path to investigate would be an ion study on $\mathrm{g}-\mathrm{C}_{3} \mathrm{~N}_{4}$ synthesised using various precursors, possibly monitoring ion absorption using UV-Vis spectroscopy. Alternatively, it would also be possible to replace $\mathrm{WO}_{3}$ with a photocatalyst which was more active in terms of oxygen evolution from water. The downside to this approach though, is searching for a photocatalyst which not only has visible light absorption and demonstrates a large OER, but also has a band gap which straddles the reduction potential of $\mathrm{IO}_{3}-$, and the redox potential of water, is indeed very difficult, but not impossible. It would also be worthy exploring the incorporation of other redox mediators into the overall water splitting systems, such as $\mathrm{NaNO}_{3}$ or chromate complexes. 


\section{Bibliography}

1 Oberthür, S. \& Ott, H. E. The Kyoto Protocol: international climate policy for the 21st century. (Springer, 1999).

2 Woodhouse, M. \& Parkinson, B. A. Combinatorial approaches for the identification and optimization of oxide semiconductors for efficient solar photoelectrolysis. Chemical Society Reviews 38, 197210, (2009).

3 Hoffmann, M. R., Martin, S. T., Choi, W. Y. \& Bahnemann, D. W. Environmental Applications of Semiconductor Photocatalysis. Chem Rev 95, 69-96, (1995).

4 Sigfusson, T. I. Pathways to hydrogen as an energy carrier Philosophical Transactions of the Royal Society A: Mathematical, Physical and Engineering Sciences 365, 1025-1042, (2007).

5 Somorjai, G. A. \& Turner, J. E. Catalyzed photodissociation of water - The first step in inorganic photosynthesis? Naturwissenschaften 71, 575-577, (1984).

6 Fujishima, A., Honda, K. \& Kohayakawa, K. Electrochemical Photolysis of Water at a Semiconductor Electrode. Nature 238, (1972).

$7 \quad$ Yi, Z. et al. An orthophosphate semiconductor with photooxidation properties under visible-light irradiation. Nature Materials 9, 559$564,(2010)$.

8 Sayama, K. et al. Photoelectrochemical Decomposition of Water into $\mathrm{H}_{2}$ and $\mathrm{O}_{2}$ on Porous $\mathrm{BiVO}_{4}$ Thin-Film Electrodes under Visible Light and Significant Effect of Ag Ion Treatment. The Journal of Physical Chemistry B 110, 11352-11360, (2006).

9 Maeda, K. et al. Photocatalytic Overall Water Splitting Promoted by Two Different Cocatalysts for Hydrogen and Oxygen Evolution under Visible Light. Angewandte Chemie-International Edition 49, 4096-4099, (2010).

10 Bard, A. J. \& Fox, M. A. Artificial Photosynthesis: Solar Splitting of Water to Hydrogen and Oxygen. Accounts of Chemical Research 28, 141-145, (1995)

11 Serpone, N. \& Pelizzetti, E. Photocatalysis: Fundamentals and Applications. (Wiley, Chichester, 1989).

12 Erbs, W., Desilvestro, J., Borgarello, E. \& Graetzel, M. Visiblelight-induced oxygen generation from aqueous dispersions of tungsten (VI) oxide. The Journal of Physical Chemistry 88, 40014006, (1984).

13 Nozik, A. J. \& Memming, R. Physical Chemistry of SemiconductorLiquid Interfaces. The Journal of Physical Chemistry 100, 1306113078, (1996).

14 Reber, J. F. \& Rusek, M. Photochemical hydrogen production with platinized suspensions of cadmium sulfide and cadmium zinc sulfide modified by silver sulfide. The Journal of Physical Chemistry 90, 824-834, (1986).

Murphy, A. B. et al. Efficiency of solar water splitting using semiconductor electrodes. International Journal of Hydrogen Energy 31, 1999-2017, (2006).

16 Gerischer, H. Electrochemical photo and solar cells principles and some experiments. Journal of Electroanalytical Chemistry and Interfacial Electrochemistry 58, 263-274, (1975).

Kudo, A., Omori, K. \& Kato, H. A novel aqueous process for preparation of crystal form-controlled and highly crystalline $\mathrm{BiVO}_{4}$ powder from layered vanadates at room temperature and its photocatalytic and photophysical properties. Journal of the American Chemical Society 121, 11459-11467, (1999). 

Visible-Light-Driven Overall Water Splitting. Journal of the American Chemical Society 127, 8286-8287, (2005).

19 Bard, A. J., Memming, R. \& Miller, B. Terminology in semiconductor electrochemistry and photoelectochemical conversion - Recommendations Pure and applied chemistry 63, 569-596, (1991). Zhang, W., Tang, J. \& Ye, J. Structural, photocatalytic, and photophysical properties of perovskite $\mathrm{MSnO}_{3} \quad(\mathrm{M}=\mathrm{Ca}, \mathrm{Sr}$, and Ba) photocatalysts. Journal of Materials Research 22, 1859-1871, (2007).

21 Bisquert, J. Physical electrochemistry of nanostructured devices. Physical Chemistry Chemical Physics 10, 49-72, (2008).

Grey, I. E. Electrochemical Water Splitting - A review of published research. CSIRO Minerals: 2004.

23 Kudo, A. \& Miseki, Y. Heterogeneous photocatalyst materials for water splitting. Chemical Society Reviews 38, 253-278, (2009).

Park, H. G. \& Holt, J. K. Recent advances in nanoelectrode architecture for photochemical hydrogen production. Energy \& Environmental Science 3, 1028-1036, (2010).

Rajeshwar, K. Hydrogen generation at irradiated oxide semiconductor-solution interfaces. Journal of Applied Electrochemistry 37, 765-787, (2007).

Jing, D. et al. Efficient solar hydrogen production by photocatalytic water splitting: From fundamental study to pilot demonstration. International Journal of Hydrogen Energy 35, 7087-7097, (2010). Varghese, O. K. \& Grimes, C. A. Appropriate strategies for determining the photoconversion efficiency of water photoelectrolysis cells: A review with examples using titania nanotube array photoanodes. Solar Energy Materials and Solar Cells 92, 374-384, (2008).

Archer, M. D. Nanostructured and photoelectrochemical systems for solar photon conversion : Series on photoconversion of solar energy. (2008).

Vayssieres, L. On Solar Hydrogen and Nanotechnology. (John Wiley \& Sons, 2009).

30 Pendlebury, S. R. et al. Dynamics of photogenerated holes in nanocrystalline $\alpha-\mathrm{Fe}_{2} \mathrm{O}_{3}$ electrodes for water oxidation probed by transient absorption spectroscopy. Chemical Communications 47, (2011)

31 Yu, J. \& Kudo, A. Effects of Structural Variation on the Photocatalytic Performance of Hydrothermally Synthesized BiVO Advanced Functional Materials 16, 2163-2169, (2006).

Parida, K. M., Reddy, K. H., Martha, S., Das, D. P. \& Biswal, N. Fabrication of nanocrystalline $\mathrm{LaFeO}_{3}$ : An efficient sol-gel autocombustion assisted visible light responsive photocatalyst for water decomposition. International Journal of Hydrogen Energy 35 , 12161-12168, (2010).

33 Allen, L. C. Electronegativity is the average one-electron energy of the valence-shell electrons in ground-state free atoms. Journal of the American Chemical Society 111, 9003-9014, (1989).

34 Mills, A. \& Valenzuela, M. A. Photo-oxidation of water sensitized by $\mathrm{TiO}_{2}$ and $\mathrm{WO}_{3}$ in presence of different electron acceptors. Rev Mex Fis 50, 287-296, (2004).

35 Mills, A. \& Valenzuela, M. A. The photo-oxidation of water by sodium persulfate, and other electron acceptors, sensitised by $\mathrm{TiO}_{2}$. Journal of Photochemistry and Photobiology A: Chemistry 165, 2534, (2004).

36 Bamwenda, G. R., Tsubota, S., Nakamura, T. \& Haruta, M. Photoassisted hydrogen production from a water-ethanol solution: a comparison of activities of $\mathrm{Au}-\mathrm{TiO}_{2}$ and $\mathrm{Pt}-\mathrm{TiO}_{2}$. Journal of Photochemistry and Photobiology A: Chemistry 89, 177-189, (1995). 
Li Puma, G. Modeling of Thin-Film Slurry Photocatalytic Reactors Affected by Radiation Scattering. Environ Sci Technol 37, 57835791, (2003).

38 Serpone, N., Terzian, R., Lawless, D., Kennepohl, P. \& Sauvé, G. On the usage of turnover numbers and quantum yields in heterogeneous photocatalysis. Journal of Photochemistry and Photobiology A: Chemistry 73, 11-16, (1993).

Weber, M. F. \& Dignam, M. J. Efficiency of splitting water with semiconducting photoelectrodes. Journal of The Electrochemical Society 131, 1258-1265, (1984).

40 Bolton, J. R., Haught, A. F. \& Ross, R. T. Photochemical energy storage: an analysis of limits. Photochemical Conversion and Storage of Solar Energy, 297-330, (1981).

41 Bolton, J. R., Strickler, S. J. \& Connolly, J. S. Limiting and realizable efficiencies of solar photolysis of water. Nature 316, 495500, (1985).

42 Tang, J., Cowan, A. J., Durrant, J. R. \& Klug, D. R. Mechanism of $\mathrm{O}_{2}$ Production from Water Splitting: Nature of Charge Carriers in Nitrogen Doped Nanocrystalline $\mathrm{TiO}_{2}$ Films and Factors Limiting $\mathrm{O}_{2}$ Production. The Journal of Physical Chemistry $C$ 115, 31433150, (2011).

43 Tauc, J. Optical properties and electronic structure of amorphous Ge and Si. Materials Research Bulletin 3, 37-46, (1968).

44 Tsunekawa, S., Sahara, R., Kawazoe, Y. \& Kasuya, A. Origin of the blue shift in ultraviolet absorption spectra of nanocrystalline $\mathrm{CeO}_{[2-}$ x] particles. 233, (2000).

45 Kadoya, K., Matsunaga, N. \& Nagashima, A. Viscosity and thermal conductivity of dry air in the gaseous phase. Journal of physical and chemical reference data 14, 947-970, (1985).

46 Bragg, W. L. The Crystalline State: Volume I. (New York: The Macmillan Company, 1934).

47 Hart, M. X-ray diffraction by L. V. Azaroff, R. Kaplow, N. Kato, R. J. Weiss, A. J. C. Wilson and R. A. Young. Acta Crystallographica Section $A$ 31, 878, (1975).

48 Bueno-Ferrer, C., Parres-Esclapez, S., Lozano-Castelló, D. \& BuenoLópez, A. Relationship between surface area and crystal size of pure and doped cerium oxides. Journal of Rare Earths 28, 647-653, (2010).

49 Egerton, R. F. Physical principles of electron microscopy: an introduction to TEM, SEM, and AEM. (Springer Science+Business Media, 2005).

50 Brunauer, S., Emmett, P. H. \& Teller, E. Adsorption of Gases in Multimolecular Layers. Journal of the American Chemical Society 60, 309-319, (1938).

51 Hanaor, D., Michelazzi, M., Leonelli, C. \& Sorrell, C. C. The effects of carboxylic acids on the aqueous dispersion and electrophoretic deposition of $\mathrm{ZrO}_{2}$. Journal of the European Ceramic Society 32 , 235-244, (2012).

52 Mirabella, F. M. Internal reflection spectroscopy: Theory and applications. Vol. 15 (CRC Press, 1992).

53 White, R. Chromatography/Fourier Transform Infrared Spectroscopy and its Applications. (Taylor \& Francis, 1989).

54 Greener, J., Abbasi, B. \& Kumacheva, E. Attenuated total reflection Fourier transform infrared spectroscopy for on-chip monitoring of solute concentrations. Lab on a Chip 10, 1561-1566, (2010).

55 Smith, E. \& Dent, G. Modern Raman spectroscopy: a practical approach. (John Wiley \& Sons, 2005).

56 Bernath, P. F. Spectra of atoms and molecules. (Oxford University Press, 2005). 
Hollander, J. M. \& Jolly, W. L. X-ray photoelectron spectroscopy. Accounts of Chemical Research 3, 193-200, (1970). Wagner, C. \& Muilenberg, G. Handbook of X-ray photoelectron spectroscopy. (Perkin-Elmer, 1979).

Moulder, J. F., Stickle, W. F., Sobol, P. E. \& Bomben, K. D. Handbook of $X$-ray photoelectron spectroscopy: a reference book of standard spectra for identification and interpretation of. (PerkinElmer: Boca Raton, FL, 1992).

60 Asahi, R., Morikawa, T., Ohwaki, T., Aoki, K. \& Taga, Y. VisibleLight Photocatalysis in Nitrogen-Doped Titanium Oxides. Science 293, 269-271, (2001).

61 Wang, J. et al. Origin of Photocatalytic Activity of Nitrogen-Doped $\mathrm{TiO}_{2}$ Nanobelts. Journal of the American Chemical Society 131, 12290-12297, (2009).

62 Ishikawa, Y., Matsumoto, Y., Nishida, Y., Taniguchi, S. \& Watanabe, J. Surface treatment of silicon carbide using $\mathrm{TiO}_{2}$ (IV) photocatalyst. Journal of the American Chemical Society 125, 65586562, (2003).

63 Kawasaki, S. et al. Epitaxial Rh-doped $\mathrm{SrTiO}_{3}$ thin film photocathode for water splitting under visible light irradiation. Appl Phys Lett 101, doi:http://dx.doi.org/10.1063/1061.4738371, (2012). Holmes, F. L. Elementary analysis and the origins of physiological chemistry. Isis, 50-81, (1963).

65 Pavia, D., Lampman, G., Kriz, G. \& Vyvyan, J. Introduction to spectroscopy. (Cengage Learning, 2008).

66 Li, K., Martin, D. J. \& Tang, J. Conversion of Solar Energy to Fuels by Inorganic Heterogeneous Systems. Chinese Journal of Catalysis 32, 879-890, (2011).

D. \& Luan, J. Development of Visible Light-Responsive Sensitized Photocatalysts. International Journal of Photoenergy 2012, 13, (2012).

68 Khaselev, O. \& Turner, J. A. A monolithic photovoltaicphotoelectrochemical device for hydrogen production via water splitting. Science 280, 425-427, (1998).

69 Yamaguti, K. \& Sato, S. Pressure dependence of the rate and stoichiometry of water photolysis over platinized $\mathrm{TiO}_{2}$ (anatase and rutile) catalysts. The Journal of Physical Chemistry 89, 5510-5513, (1985).

70 Selli, E. et al. A photocatalytic water splitting device for separate hydrogen and oxygen evolution. Chemical Communications, 50225024, (2007).

71 Duonghong, D. Dynamics of light-induced water cleavage in colloidal systems. Journal of the American Chemical Society 103 , 4685, (1981).

72 Sato, S. \& White, J. M. Photoassisted hydrogen production from titania and water. The Journal of Physical Chemistry 85, 592-594, (1981).

73 Fu, N., Wu, Y., Jin, Z. \& Lu, G. Structural-Dependent photoactivities of $\mathrm{TiO}_{2}$ nanoribbon for Visible-Light-Induced $\mathrm{H}_{2}$ Evolution: The roles of nanocavities and alternate structures. Langmuir 26, 447-455, (2010).

74 Li, Y., Xie, Y., Peng, S., Lu, G. \& Li, S. Photocatalytic hydrogen generation in the presence of chloroacetic acids over $\mathrm{Pt} / \mathrm{TiO}_{2}$. Chemosphere 63, 1312-1318, (2006).

75 Domen, K. Photocatalytic decomposition of water into hydrogen and oxygen over nickel (II) oxide-strontium titanate $\left(\mathrm{SrTiO}_{3}\right)$ powder. Journal of physical chemistry 90, 292, (1986).

Domen, K., Kudo, A. \& Onishi, T. Mechanism of photocatalytic decomposition of water into $\mathrm{H}_{2}$ and $\mathrm{O}_{2}$ over $\mathrm{NiO}-\mathrm{SrTiO}_{3}$. Journal of Catalysis 102, 92-98, (1986). 

decomposition of liquid water on a $\mathrm{NiO}_{-} \mathrm{SrTiO}_{3}$ catalyst. Chemical Physics Letters 92, 433-434, (1982).

78 Kudo, A. et al. Nickel-loaded $\mathrm{K}_{4} \mathrm{Nb}_{6} \mathrm{O}_{17}$ photocatalyst in the decomposition of $\mathrm{H}_{2} \mathrm{O}$ into $\mathrm{H}_{2}$ and $\mathrm{O}_{2}$ : Structure and reaction mechanism. Journal of Catalysis 120, 337-352, (1989).

79 Kudo, A. et al. Photocatalytic decomposition of water over NiO$\mathrm{K}_{4} \mathrm{Nb}_{6} \mathrm{O}_{17}$ catalyst. Journal of Catalysis 111, 67-76, (1988).

80 Ji, S. M. \& Min, J. Photocatalytic hydrogen production from water methanol mixtures using $\mathrm{N}$-doped $\mathrm{Sr}_{2} \mathrm{Nb}_{2} \mathrm{O}_{7}$ under visible light irradiation: effects of catalyst structure. Physical chemistry chemical physics 7, 1315, (2005).

81 Chang, S. \& Doong, R. The Effect of Chemical States of Dopants on the Microstructures and Band Gaps of Metal-Doped $\mathrm{ZrO}_{2}$ Thin Films at Different Temperatures. The Journal of Physical Chemistry $B$ 108, 18098-18103, (2004).

82 Sayama, K. \& Arakawa, H. Photocatalytic decomposition of water and photocatalytic reduction of carbon dioxide over zirconia catalyst. The Journal of Physical Chemistry 97, 531-533, (1993).

Jiang, L., Wang, Q., Li, C., Yuan, J. \& Shangguan, W. ZrW ${ }_{2} \mathrm{O}_{8}$ photocatalyst and its visible-light sensitization via sulfur anion doping for water splitting. International Journal of Hydrogen Energy 35, 7043-7050, (2010).

84 Sayama, K. Photocatalytic water splitting on nickel intercalated $\mathrm{A}_{4} \mathrm{Ta}_{\mathrm{x}} \mathrm{Nb}_{6-\mathrm{x}} \mathrm{O}_{17}(\mathrm{~A}=\mathrm{K}, \mathrm{Rb})$. Catalysis today 28, 175, (1996).

85 Kato, H. New tantalate photocatalysts for water decomposition into $\mathrm{H}_{2}$ and $\mathrm{O}_{2}$. Chemical Physics Letters 295, 487, (1998).

86 Kato, $\mathrm{H}$. Water splitting into $\mathrm{H}_{2}$ and $\mathrm{O}_{2}$ on alkali tantalate photocatalysts $\mathrm{ATaO}_{3}(\mathrm{~A}=\mathrm{Li}, \mathrm{Na}$, and $\mathrm{K})$. The Journal of Physical Chemistry $B$ 105, 4285, (2001).

87 Kato, H., Asakura, K. \& Kudo, A. Highly Efficient Water Splitting into $\mathrm{H}_{2}$ and $\mathrm{O}_{2}$ over Lanthanum-Doped $\mathrm{NaTaO}_{3}$ Photocatalysts with High Crystallinity and Surface Nanostructure. Journal of the American Chemical Society 125, 3082-3089, (2003).

88 Mitsui, C., Nishiguchi, H., Fukamachi, K., Ishihara, T. \& Takita, Y. Photocatalytic Decomposition of Pure Water over NiO Supported on $\mathrm{KTa}(\mathrm{M}) \mathrm{O}_{3}\left(\mathrm{M}=\mathrm{Ti}^{4+}, \mathrm{Hf}^{4+}, \mathrm{Zr}^{4+}\right)$ Perovskite Oxide Chemistry Letters 28, 1327-1328, (1999).

89 Yoshino, M. Polymerizable Complex Synthesis of Pure $\mathrm{Sr}_{2} \mathrm{Nb}_{\mathrm{x}} \mathrm{Ta}_{2}$ ${ }_{x} \mathrm{O}_{7}$ Solid Solutions with High Photocatalytic Activities for Water Decomposition into $\mathrm{H}_{2}$ and $\mathrm{O}_{2}$. Chemistry of materials 14, 3369, (2002).

90 Domen, K. et al. Photodecomposition of water and hydrogen evolution from aqueous methanol solution over novel niobate photocatalysts. Journal of the Chemical Society, Chemical Communications, 356-357, (1986).

91 Wiegelm, Emond, M. H. J., Stobbe, E. R. \& Blasse, G. Luminescence of Alkali Tantalates and Niobates. Journal of Physics and Chemistry of Solids 55, 773-778, (1994).

92 Sato, J., Kobayashi, H. \& Inoue, Y. Photocatalytic Activity for Water Decomposition of Indates with Octahedrally Coordinated d10 Configuration. II. Roles of Geometric and Electronic Structures. The Journal of Physical Chemistry B 107, 7970-7975, (2003).

93 Sato, J. Photocatalytic activities for water decomposition of $\mathrm{RuO}_{2^{-}}$ loaded $\mathrm{AInO}_{2}(\mathrm{~A}=\mathrm{Li}, \mathrm{Na})$ with d10 configuration. Journal of photochemistry and photobiology. A, Chemistry 158, 139, (2003).

94 Zhang, W. F., Tang, J. \& Ye, J. Photoluminescence and photocatalytic properties of $\mathrm{SrSnO}_{3}$ perovskite. Chemical Physics Letters 418, 174-178, (2006). 
Chen, D. \& Ye, J. $\mathrm{SrSnO}_{3}$ Nanostructures: Synthesis, Characterization, and Photocatalytic Properties. Chemistry of materials 19, 4585-4591, (2007).

96 Sato, J., Saito, N., Nishiyama, H. \& Inoue, Y. Photocatalytic water decomposition by $\mathrm{RuO}_{2}$-loaded antimonates, $\mathrm{M}_{2} \mathrm{Sb}_{2} \mathrm{O}_{7}(\mathrm{M}=\mathrm{Ca}, \mathrm{Sr})$, $\mathrm{CaSb}_{2} \mathrm{O}_{6}$ and $\mathrm{NaSbO}_{3}$, with d10 configuration. Journal of Photochemistry and Photobiology A: Chemistry 148, 85-89, (2002). Yanagida, T. Photocatalytic Decomposition of $\mathrm{H}_{2} \mathrm{O}$ into $\mathrm{H}_{2}$ and $\mathrm{O}_{2}$ over $\mathrm{Ga}_{2} \mathrm{O}_{3}$ Loaded with $\mathrm{NiO}$. Chemistry Letters 33, 726, (2004).

98 Sakata, Y. Effect of Metal Ion Addition in a Ni Supported $\mathrm{Ga}_{2} \mathrm{O}_{3}$ Photocatalyst on the Photocatalytic Overall Splitting of $\mathrm{H}_{2} \mathrm{O}$. Catalysis letters 125, 22, (2008).

99 Ikarashi, K. Photocatalysis for Water Decomposition by $\mathrm{RuO}_{2^{-}}$ Dispersed $\mathrm{ZnGa}_{2} \mathrm{O}_{4}$ with d10 Configuration. The journal of physical chemistry. B 106, 9048, (2002).

100 Yanagida, S. Photocatalytic hydrogen evolution from water using zinc sulfide and sacrificial electron donors. Chemistry Letters 11, 1069, (1982).

101 Reber, J. F. \& Meier, K. Photochemical production of hydrogen with zinc sulfide suspensions. The Journal of Physical Chemistry 88 , 5903-5913, (1984).

102 Kobayakawa, K. Photocatalytic activity of $\mathrm{CuInS}_{2}$ and $\mathrm{CuIn}_{5} \mathrm{~S}_{8}$. Electrochimica Acta 37, 465, (1992).

103 Sato, J. et al. $\mathrm{RuO}_{2}$-loaded beta- $\mathrm{Ge}_{3} \mathrm{~N}_{4}$ as a non-oxide photocatalyst for overall water splitting. Journal of the American Chemical Society 127, 4150-4151, (2005).

104 Lee, Y. G. et al. Effect of high-pressure ammonia treatment on the activity of $\mathrm{Ge}_{3} \mathrm{~N}_{4}$ photocatalyst for overall water splitting. $J$ Phys Chem B 110, 17563-17569, (2006).

105 Maeda, K., Saito, N., Inoue, Y. \& Domen, K. Dependence of activity and stability of germanium nitride powder for photocatalytic overall water splitting on structural properties. Chemistry of materials 19, 4092-4097, (2007).

106 Maeda, K., Teramura, K., Saito, N., Inoue, Y. \& Domen, K. Photocatalytic overall water splitting on gallium nitride powder. $B$ Chem Soc Jpn 80, 1004-1010, (2007).

107 Arai, N. et al. Overall water splitting by $\mathrm{RuO}_{2}$-dispersed divalention-doped GaN photocatalysts with d(10) electronic configuration. Chemistry Letters 35, 796-797, (2006).

108 Wei, S. H. \& Zunger, A. Role of metal d states in II-VI semiconductors. Physical Review B 37, 8958, (1988).

109 Kawai, T. \& Sakata, T. Photocatalytic hydrogen production from liquid methanol and water. Journal of the Chemical Society, Chemical Communications, 694-695, (1980).

110 Kawai, T. \& Sakata, T. Conversion of carbohydrate into hydrogen fuel by a photocatalytic process. (1980).

111 Sakata, T. \& Kawai, T. Heterogeneous photocatalytic production of hydrogen and methane from ethanol and water. Chemical Physics Letters 80, 341-344, (1981).

112 Borgarello, E., Kiwi, J., Graetzel, M., Pelizzetti, E. \& Visca, M. Visible light induced water cleavage in colloidal solutions of chromium-doped titanium dioxide particles. Journal of the American Chemical Society 104, 2996-3002, (1982).

113 Chen, X. \& Mao, S. S. Titanium Dioxide Nanomaterials: Synthesis, Properties, Modifications, and Applications. Chem Rev 107, 28912959, (2007).

$114 \mathrm{Ji}, \mathrm{P}$. et al. Recent advances in visible light-responsive titanium oxide-based photocatalysts. Res Chem Intermediat 36, 327-347, (2010).

115 Leung, D. Y. C. et al. Hydrogen Production over Titania-Based Photocatalysts. ChemSusChem 3, 681-694, (2010). 
116 Anpo, M. et al. The design and development of second-generation titanium oxide photocatalysts able to operate under visible light irradiation by applying a metal ion-implantation method. Res Chem Intermediat 27, 459-467, (2001).

117 Choi, W., Termin, A. \& Hoffmann, M. R. The Role of Metal Ion Dopants in Quantum-Sized $\mathrm{TiO}_{2}$ : Correlation between Photoreactivity and Charge Carrier Recombination Dynamics. The Journal of Physical Chemistry 98, 13669-13679, (1994).

118 Rengaraj, S. \& Li, X. Enhanced photocatalytic activity of $\mathrm{TiO}_{2}$ by doping with $\mathrm{Ag}$ for degradation of 2, 4, 6-trichlorophenol in aqueous suspension. Journal of Molecular Catalysis A: Chemical 243, 60-67, (2006).

119 Kim, S., Hwang, S.-J. \& Choi, W. Visible Light Active PlatinumIon-Doped $\mathrm{TiO}_{2}$ Photocatalyst. The Journal of Physical Chemistry $B$ 109, 24260-24267, (2005).

120 Umebayashi, T., Yamaki, T., Itoh, H. \& Asai, K. Band gap narrowing of titanium dioxide by sulfur doping. Appl Phys Lett 81, 454-456, (2002).

121 Choi, Y., Umebayashi, T. \& Yoshikawa, M. Fabrication and characterization of C-doped anatase $\mathrm{TiO}_{2}$ photocatalysts. Journal of Materials Science 39, 1837-1839, (2004).

122 Reyes-Garcia, E. A., Sun, Y. \& Raftery, D. Solid-state characterization of the nuclear and electronic environments in a boron-fluoride co-doped $\mathrm{TiO}_{2}$ visible-light photocatalyst. The Journal of Physical Chemistry C 111, 17146-17154, (2007).

123 Konta, R., Ishii, T., Kato, H. \& Kudo, A. Photocatalytic activities of noble metal ion doped $\mathrm{SrTiO}_{3}$ under visible light irradiation. The Journal of Physical Chemistry B 108, 8992-8995, (2004).

124 Nishimoto, S., Matsuda, M. \& Miyake, M. Photocatalytic activities of Rh-doped $\mathrm{CaTiO}_{3}$ under visible light irradiation. Chemistry Letters 35, 308-309, (2006).

125 Okazaki, Y., Mishima, T., Nishimoto, S., Matsuda, M. \& Miyake, M. Photocatalytic activity of $\mathrm{Ca}_{3} \mathrm{Ti}_{2} \mathrm{O}_{7}$ layered-perovskite doped with $\mathrm{Rh}$ under visible light irradiation. Mater Lett 62, 3337-3340, (2008).

126 Zou, Z., Ye, J., Sayama, K. \& Arakawa, H. Direct splitting of water under visible light irradiation with an oxide semiconductor photocatalyst. Nature 414, 625-627, (2001).

127 Iwase, A., Saito, K. \& Kudo, A. Sensitization of $\mathrm{NaMO}_{3}(\mathrm{M}: \mathrm{Nb}$ and Ta) photocatalysts with wide band gaps to visible light by Ir doping. $B$ Chem Soc Jpn 82, 514-518, (2009).

128 Hara, M. TaON and $\mathrm{Ta}_{3} \mathrm{~N}_{5}$ as new visible light driven photocatalysts. Catalysis today 78, 555, (2003).

129 Hara, M., Nunoshige, J., Takata, T., Kondo, J. N. \& Domen, K. Unusual enhancement of $\mathrm{H}_{2}$ evolution by $\mathrm{Ru}$ on $\mathrm{TaON}$ photocatalyst under visible light irradiation. Chemical Communications, 30003001, (2003).

130 Liu, M. et al. Water reduction and oxidation on $\mathrm{Pt}-\mathrm{Ru} / \mathrm{Y}_{2} \mathrm{Ta}_{2} \mathrm{O}_{5} \mathrm{~N}_{2}$ catalyst under visible light irradiation. Chemical Communications, 2192-2193, (2004).

131 Yamasita, D., Takata, T., Hara, M., Kondo, J. N. \& Domen, K. Recent progress of visible-light-driven heterogeneous photocatalysts for overall water splitting. Solid state ionics 172, 591-595, (2004).

132 Maeda, K., Teramura, K. \& Domen, K. Effect of post-calcination on photocatalytic activity of $\left(\mathrm{Ga}_{1-\mathrm{x}} \mathbf{Z n}_{\mathrm{x}}\right)\left(\mathbf{N}_{1-\mathrm{x}} \mathrm{O}_{\mathrm{x}}\right)$ solid solution for overall water splitting under visible light. Journal of Catalysis 254, 198-204, (2008).

133 Maeda, K. et al. Photocatalyst releasing hydrogen from water. Nature 440, 295-295, (2006). 
134 Tang, J. Correlation of crystal structures and electronic structures and photocatalytic properties of the $\mathrm{W}$-containing oxides. Journal of materials chemistry 15, 4246, (2005).

135 Tang, J. Photophysical and photocatalytic properties of $\mathrm{AgInW}_{2} \mathrm{O}_{8}$. the Journal of physical Chemistry $B$ 107, 14265, (2003).

136 Kudo, A. Photocatalytic $\mathrm{O}_{2}$ evolution under visible light irradiation on $\mathrm{BiVO}_{4}$ in aqueous $\mathrm{AgNO}_{3}$ solution. Catalysis letters 53, 229, (1998).

137 Darwent, J. R. $\mathbf{H}_{2}$ production photosensitized by aqueous semiconductor dispersions. Journal of the Chemical Society, Faraday Transactions 2: Molecular and Chemical Physics 77, 17031709, (1981).

138 Darwent, J. R. \& Porter, G. Photochemical hydrogen production using cadmium sulphide suspensions in aerated water. Journal of the Chemical Society, Chemical Communications, 145-146, (1981).

139 Yan, H. et al. Visible-light-driven hydrogen production with extremely high quantum efficiency on Pt-PdS/CdS photocatalyst. Journal of Catalysis 266, 165-168, (2009).

140 Ma, G. et al. Direct splitting of $\mathrm{H}_{2} \mathrm{~S}$ into $\mathrm{H}_{2}$ and $\mathrm{S}$ on CdS-based photocatalyst under visible light irradiation. Journal of Catalysis 260, 134-140, (2008).

141 Tabata, M. et al. Photocatalytic hydrogen evolution from water using copper gallium sulfide under visible-light irradiation. The Journal of Physical Chemistry C 114, 11215-11220, (2010).

142 Zong, X. et al. Enhancement of photocatalytic $\mathrm{H}_{2}$ evolution on CdS by loading $\mathrm{MoS}_{2}$ as cocatalyst under visible light irradiation. Journal of the American Chemical Society 130, 7176-7177, (2008).

143 Kalyanasundaram, K., Borgarello, E., Duonghong, D. \& Grätzel, M. Cleavage of Water by Visible-Light Irradiation of Colloidal CdS Solutions; Inhibition of Photocorrosion by $\mathrm{RuO}_{2}$. Angewandte Chemie International Edition in English 20, 987-988, (1981).

144 Youn, H. C., Baral, S. \& Fendler, J. H. Dihexadecyl Phosphate, Vesicle-Stabilized and Insitu Generated Mixed CdS and ZnS Semiconductor Particles - Preparation and Utilization for Photosensitized Charge Separation and Hydrogen Generation. Journal of physical chemistry 92, 6320-6327, (1988).

145 Sabate, J., Cerveramarch, S., Simarro, R. \& Gimenez, J. A Comparative-Study of Semiconductor Photocatalysts for HydrogenProduction by Visible-Light Using Different Sacrificial Substrates in Aqueous-Media. International Journal of Hydrogen Energy 15, 115-124, (1990).

146 Xing, C. J., Zhang, Y. J., Yan, W. \& Guo, L. J. And structurecontrolled solid solution of $\mathrm{Cd}_{1-\mathrm{x}} \mathrm{Zn}_{\mathrm{x}} \mathrm{S}$ photocatalyst for hydrogen production by water splitting. International Journal of Hydrogen Energy 31, 2018-2024, (2006).

147 Rufus, I. B., Viswanathan, B., Ramakrishnan, V. \& Kuriacose, J. C. Cadmium-Sulfide with Iridium Sulfide and Platinum Sulfide Deposits as a Photocatalyst for the Decomposition of Aqueous Sulfide. J Photoch Photobio A 91, 63-66, (1995).

148 Umezawa, N., Shuxin, O. \& Ye, J. Theoretical study of high photocatalytic performance of $\mathrm{Ag}_{3} \mathrm{PO}_{4}$. Physical Review B 83, 035202, (2011).

149 Bi, Y., Ouyang, S., Umezawa, N., Cao, J. \& Ye, J. Facet Effect of Single-Crystalline $\mathrm{Ag}_{3} \mathrm{PO}_{4}$ Sub-microcrystals on Photocatalytic Properties. Journal of the American Chemical Society 133, 64906492, (2011).

150 Bi, Y., Ouyang, S., Cao, J. \& Ye, J. Facile synthesis of rhombic dodecahedral $\mathrm{AgX} / \mathrm{Ag}_{3} \mathrm{PO}_{4}(\mathrm{X}=\mathrm{Cl}, \mathrm{Br}, \mathrm{I})$ heterocrystals with enhanced photocatalytic properties and stabilities. Physical Chemistry Chemical Physics 13, 10071-10075, (2011). 
151 Wang, X. et al. A metal-free polymeric photocatalyst for hydrogen production from water under visible light. Nature Materials 8, 7680, (2008).

152 Liebig, J. Ueber Einige Stickstoff-Verbindungen. Ann. Pharm. 10, 1-47, (1834).

153 Franklin, E. C. The ammono carbonic acids. Journal of the American Chemical Society 44, 486-509, (1922).

154 Pauling, L. \& Sturdivant, J. H. The Structure of Cyameluric Acid, Hydromelonic Acid and Related Substances. Proceedings of the National Academy of Sciences 23, 615-620, (1937).

155 Lotsch, B. V. et al. Unmasking Melon by a Complementary Approach Employing Electron Diffraction, Solid-State NMR Spectroscopy, and Theoretical Calculations-Structural Characterization of a Carbon Nitride Polymer. Chemistry - A European Journal 13, 4969-4980, (2007).

156 Kroke, E. \& Schwarz, M. Novel group 14 nitrides. Coordination Chemistry Reviews 248, 493-532, (2004).

157 Kroke, E. et al. Tri-s-triazine derivatives. Part I. From trichloro-tris-triazine to graphitic $\mathrm{C}_{3} \mathrm{~N}_{4}$ structures. New Journal of Chemistry 26, 508-512, (2002).

158 Dong, F., Sun, Y., Wu, L., Fu, M. \& Wu, Z. Facile transformation of low cost thiourea into nitrogen-rich graphitic carbon nitride nanocatalyst with high visible light photocatalytic performance. Catalysis Science \& Technology 2, 1332-1335, (2012).

159 Dong, F. et al. Efficient synthesis of polymeric $\mathrm{g}-\mathrm{C}_{3} \mathrm{~N}_{4}$ layered materials as novel efficient visible light driven photocatalysts. Journal of Materials Chemistry 21, 15171-15174, (2011).

160 Yue, B., Li, Q., Iwai, H., Kako, T. \& Ye, J. Hydrogen production using zinc-doped carbon nitride catalyst irradiated with visible light. Science and Technology of Advanced Materials 12, 034401, (2011).

161 Jorge, A. B. et al. $\mathrm{H}_{2}$ and $\mathrm{O}_{2}$ Evolution from Water Half-Splitting Reactions by Graphitic Carbon Nitride Materials. The Journal of Physical Chemistry C 117, 7178-7185, (2013).

162 Ge, L., Han, C., Xiao, X., Guo, L. \& Li, Y. Enhanced visible light photocatalytic hydrogen evolution of sulfur-doped polymeric $\mathrm{g}-\mathrm{C}_{3} \mathrm{~N}_{4}$ photocatalysts. Materials Research Bulletin.

163 Schwinghammer, K. et al. Triazine-based Carbon Nitrides for Visible-Light-Driven Hydrogen Evolution. Angewandte Chemie International Edition 52, 2435-2439, (2013).

164 Xiang, Q., Yu, J. \& Jaroniec, M. Preparation and Enhanced VisibleLight Photocatalytic $\mathbf{H}_{2}$-Production Activity of Graphene $/ \mathbf{C}_{3} \mathbf{N}_{4}$ Composites. The Journal of Physical Chemistry C 115, 7355-7363, (2011).

165 Wang, X. et al. Polymer semiconductors for artificial photosynthesis: hydrogen evolution by mesoporous graphitic carbon nitride with visible light. Journal of the American Chemical Society 131, 1680-1681, (2009).

166 Xu, J., Li, Y., Peng, S., Lu, G. \& Li, S. Eosin Y-sensitized graphitic carbon nitride fabricated by heating urea for visible light photocatalytic hydrogen evolution: the effect of the pyrolysis temperature of urea. Physical Chemistry Chemical Physics 15, 76577665, (2013).

167 Wang, Y., Zhang, J., Wang, X., Antonietti, M. \& Li, H. Boron-and Fluorine-Containing Mesoporous Carbon Nitride Polymers: MetalFree Catalysts for Cyclohexane Oxidation. Angewandte Chemie International Edition 49, 3356-3359, (2010).

168 Liu, G. et al. Unique electronic structure induced high photoreactivity of sulfur-doped graphitic $\mathrm{C}_{3} \mathrm{~N}_{4}$. Journal of the American Chemical Society 132, 11642-11648, (2010). 
Bard, A. J. Photoelectrochemistry and heterogeneous photocatalysis at semiconductors. Journal of Photochemistry 10, 59-75, (1979).

170 Kato, H., Hori, M., Konta, R., Shimodaira, Y. \& Kudo, A. Construction of Z-scheme Type Heterogeneous Photocatalysis Systems for Water Splitting into $\mathrm{H}_{2}$ and $\mathrm{O}_{2}$ under Visible Light Irradiation. Chemistry Letters 33, 1348-1349, (2004).

171 Bowker, M. Sustainable hydrogen production by the application of ambient temperature photocatalysis. Green Chemistry 13, 22352246, (2011).

172 Martin, D. J., Reardon, P. J. T., Handoko, A. D. \& Tang, J. Visible light-driven pure water splitting by a nature-inspired organic semiconductor system. Journal of the American Chemical Society (In review), (2014)

173 Darwent, J. R. \& Mills, A. Photo-oxidation of water sensitized by $\mathrm{WO}_{3}$ powder. Journal of the Chemical Society, Faraday Transactions 2: Molecular and Chemical Physics 78, 359-367, (1982).

174 Abe, R., Sayama, K., Domen, K. \& Arakawa, H. A new type of water splitting system composed of two different $\mathrm{TiO}_{2}$ photocatalysts (anatase, rutile) and a $\mathrm{IO}_{3}{ }^{-} / \mathrm{I}^{-}$shuttle redox mediator. Chemical Physics Letters 344, 339-344, (2001).

175 Kim, Y. I., Salim, S., Huq, M. J. \& Mallouk, T. E. Visible-light photolysis of hydrogen iodide using sensitized layered semiconductor particles. Journal of the American Chemical Society 113, 9561-9563, (1991).

176 Sayama, K., Mukasa, K., Abe, R., Abe, Y. \& Arakawa, H. Stoichiometric water splitting into $\mathrm{H}_{2}$ and $\mathrm{O}_{2}$ using a mixture of two different photocatalysts and an $\mathrm{IO}_{3}^{-} / \mathrm{I}^{-}$shuttle redox mediator under visible light irradiation. Chemical Communications, 24162417, (2001).

177 Ohno, T., Haga, D., Fujihara, K., Kaizaki, K. \& Matsumura, M. Unique effects of iron (III) ions on photocatalytic and photoelectrochemical properties of titanium dioxide. The Journal of Physical Chemistry B 101, 6415-6419, (1997).

178 Abe, R., Sayama, K. \& Sugihara, H. Development of New Photocatalytic Water Splitting into $\mathrm{H}_{2}$ and $\mathrm{O}_{2}$ using Two Different Semiconductor Photocatalysts and a Shuttle Redox Mediator $\mathrm{IO}_{3}$ /.-. The Journal of Physical Chemistry B 109, 16052-16061, (2005).

179 Tabata, M. et al. Modified $\mathrm{Ta}_{3} \mathrm{~N}_{5}$ powder as a photocatalyst for $\mathrm{O}_{2}$ evolution in a two-step water splitting system with an iodate/iodide shuttle redox mediator under visible light. Langmuir 26, 9161-9165, (2010).

180 Maeda, K., Higashi, M., Lu, D., Abe, R. \& Domen, K. Efficient nonsacrificial water splitting through two-step photoexcitation by visible light using a modified oxynitride as a hydrogen evolution photocatalyst. Journal of the American Chemical Society 132, 5858$5868,(2010)$.

181 Maeda, K. Z-Scheme Water Splitting Using Two Different Semiconductor Photocatalysts. ACS Catalysis 3, 1486-1503, (2013).

182 Sayama, K. et al. Photocatalytic decomposition of water into $\mathrm{H}_{2}$ and $\mathrm{O}_{2}$ by a two-step photoexcitation reaction using a $\mathrm{WO}_{3}$ suspension catalyst and an $\mathrm{Fe}^{3+} / \mathrm{Fe}^{2+}$ redox system. Chemical Physics Letters 277, 387-391, (1997).

183 Hara, S. \& Irie, H. Band structure controls of $\mathrm{SrTiO}_{3}$ towards twostep overall water splitting. Applied Catalysis B: Environmental 115, 330-335, (2012).

184 Hara, S. et al. Hydrogen and oxygen evolution photocatalysts synthesized from strontium titanate by controlled doping and their performance in two-step overall water splitting under visible light. The Journal of Physical Chemistry C 116, 17458-17463, (2012). 
185 Sayama, K., Abe, R., Arakawa, H. \& Sugihara, H. Decomposition of water into $\mathrm{H}_{2}$ and $\mathrm{O}_{2}$ by a two-step photoexcitation reaction over a $\mathrm{Pt}-\mathrm{TiO}_{2}$ photocatalyst in $\mathrm{NaNO}_{2}$ and $\mathrm{Na}_{2} \mathrm{CO}_{3}$ aqueous solution. Catalysis Communications 7, 96-99, (2006).

186 Sasaki, Y., Iwase, A., Kato, H. \& Kudo, A. The effect of co-catalyst for Z-scheme photocatalysis systems with an $\mathbf{F e}^{3+} / \mathbf{F e}^{2+}$ electron mediator on overall water splitting under visible light irradiation. Journal of Catalysis 259, 133-137, (2008).

187 Sasaki, Y., Nemoto, H., Saito, K. \& Kudo, A. Solar water splitting using powdered photocatalysts driven by Z-schematic interparticle electron transfer without an electron mediator. The Journal of Physical Chemistry C 113, 17536-17542, (2009).

188 Iwase, A., Ng, Y. H., Ishiguro, Y., Kudo, A. \& Amal, R. Reduced graphene oxide as a solid-state electron mediator in Z-scheme photocatalytic water splitting under visible light. Journal of the American Chemical Society 133, 11054-11057, (2011).

189 Abe, R., Takata, T., Sugihara, H. \& Domen, K. Photocatalytic overall water splitting under visible light by $\mathrm{TaON}$ and $\mathrm{WO}_{3}$ with an $\mathrm{IO}_{3}^{-} / \mathrm{I}^{-}$shuttle redox mediator. Chemical Communications, 3829$3831,(2005)$.

190 Abe, R., Higashi, M. \& Domen, K. Overall Water Splitting under Visible Light through a Two-Step Photoexcitation between TaON and $\mathrm{WO}_{3}$ in the Presence of an Iodate-Iodide Shuttle Redox Mediator. ChemSusChem 4, 228-237, (2011).

191 Higashi, M., Abe, R., Ishikawa, A., Takata, T. \& Ohtani, B. Zscheme Overall Water Splitting on Modified-TaON Photocatalysts under Visible Light (500 nm). Chemistry Letters 37, 138-139, (2008).

192 Maeda, K. et al. Surface modification of $\mathrm{TaON}$ with monoclinic $\mathrm{ZrO}_{2}$ to produce a composite photocatalyst with enhanced hydrogen evolution activity under visible light. B Chem Soc Jpn 81, 927-937, (2008).

193 Maeda, K., Abe, R. \& Domen, K. Role and function of ruthenium species as promoters with TaON-based photocatalysts for oxygen evolution in two-step water splitting under visible light. The Journal of Physical Chemistry C 115, 3057-3064, (2011).

194 Hodes, G., Albu-Yaron, A., Decker, F. \& Motisuke, P. Threedimensional quantum-size effect in chemically deposited cadmium selenide films. Physical Review $B$ 36, 4215, (1987).

195 Furukawa, S. \& Miyasato, T. Quantum size effects on the optical band gap of microcrystalline Si:H. Physical Review $B$ 38, 5726, (1988).

196 Saremi-Yarahmadi, S., Vaidhyanathan, B. \& Wijayantha, K. G. U. Microwave-assisted low temperature fabrication of nanostructured alpha- $\mathrm{Fe}_{2} \mathrm{O}_{3}$ electrodes for solar-driven hydrogen generation. International Journal of Hydrogen Energy 35, 10155-10165, (2010).

197 Ziegler, J., Merkulov, A., Grabolle, M., Resch-Genger, U. \& Nann, T. High-quality ZnS shells for CdSe nanoparticles: Rapid microwave synthesis. Langmuir 23, 7751-7759, (2007).

198 Wang, X., Zhuang, J., Peng, Q. \& Li, Y. D. A general strategy for nanocrystal synthesis. Nature 437, 121-124, (2005).

199 Song, H., Cai, P., Huabing, Y. \& Yan, C. Hydrothermal synthesis of flaky crystallized $\mathrm{La}_{2} \mathrm{Ti}_{2} \mathrm{O}_{7}$ for producing hydrogen from photocatalytic water splitting. Catalysis letters 113, 54-58, (2007).

200 Haynes, C. L., McFarland, A. D. \& Duyne, R. P. V. SurfaceEnhanced Raman Spectroscopy. Analytical Chemistry 77, 338 A346 A, (2005).

201 Wiley, B. J. et al. Synthesis and Electrical Characterization of Silver Nanobeams. Nano Letters 6, 2273-2278, (2006). 
202 Aslam, M., Bhobe, R., Alem, N., Donthu, S. \& Dravid, V. P. Controlled large-scale synthesis and magnetic properties of singlecrystal cobalt nanorods. J A ppl Phys 98, 1-8, (2005).

203 Somorjai, G. A. \& Blakely, D. W. Mechanism of catalysis of hydrocarbon reactions by platinum surfaces. Nature 258, 580-583, (1975).

204 Yang, H. G. et al. Anatase $\mathrm{TiO}_{2}$ single crystals with a large percentage of reactive facets. Nature 453, 638-641, (2008).

205 Liu, G. et al. Enhanced photoactivity of oxygen-deficient anatase $\mathrm{TiO}_{2}$ sheets with dominant $\{001\}$ facets. The Journal of Physical Chemistry $C$ 113, 21784-21788, (2009).

206 Han, X., Kuang, Q., Jin, M., Xie, Z. \& Zheng, L. Synthesis of titania nanosheets with a high percentage of exposed (001) facets and related photocatalytic properties. Journal of the American Chemical Society 131, 3152-3153, (2009).

207 Xia, Y. N., Xiong, Y. J., Lim, B. \& Skrabalak, S. E. ShapeControlled Synthesis of Metal Nanocrystals: Simple Chemistry Meets Complex Physics? Angewandte Chemie-International Edition 48, 60-103, (2009).

208 Xi, G. C. \& Ye, J. H. Synthesis of bismuth vanadate nanoplates with exposed $\{001\}$ facets and enhanced visible-light photocatalytic properties. Chemical Communications 46, 1893-1895, (2010).

209 Chen, X., Shen, S., Guo, L. \& Mao, S. S. Semiconductor-based photocatalytic hydrogen generation. Chem Rev 110, 6503-6570, (2010).

210 Cabrera, M. I., Alfano, O. M. \& Cassano, A. E. Novel Reactor for Photocatalytic Kinetic Studies. Industrial \& Engineering Chemistry Research 33, 3031-3042, (1994).

211 Tsederberg, N. V. \& Cess, R. D. Thermal conductivity of gases and liquids. (MIT Press Cambridge, Massachusetts, 1965).

212 Seber, G. A. \& Lee, A. J. Linear regression analysis. Vol. 936 (John Wiley \& Sons, 2012).

213 Martin, D. J., Umezawa, N., Chen, X., Ye, J. \& Tang, J. Facet engineered $\mathrm{Ag}_{3} \mathrm{PO}_{4}$ for efficient water photooxidation. Energy \& Environmental Science 6, 3380-3386, (2013).

214 Reece, S. Y. et al. Wireless Solar Water Splitting Using SiliconBased Semiconductors and Earth-Abundant Catalysts. Science 334, 645-648, (2011).

215 Tang, J., Durrant, J. R. \& Klug, D. R. Mechanism of Photocatalytic Water Splitting in $\mathrm{TiO}_{2}$. Reaction of Water with Photoholes, Importance of Charge Carrier Dynamics, and Evidence for FourHole Chemistry. Journal of the American Chemical Society 130, 13885-13891, (2008).

216 Buckley, J. J., Lee, A. F., Olivi, L. \& Wilson, K. Hydroxyapatite supported antibacterial $\mathrm{Ag}_{3} \mathrm{PO}_{4}$ nanoparticles. Journal of materials chemistry 20, 8056-8063, (2010).

$217 \mathrm{Hu}, \mathrm{H}$. et al. Facile synthesis of tetrahedral $\mathrm{Ag}_{3} \mathrm{PO}_{4}$ submicrocrystals with enhanced photocatalytic properties. Journal of Materials Chemistry A 1, 2387-2390, (2013).

218 Wang, H. et al. A Facile Way to Rejuvenate $\operatorname{Ag}_{3} \mathrm{PO}_{4}$ as a Recyclable Highly Efficient Photocatalyst. Chemistry - A European Journal 18, 5524-5529, (2012).

219 Chen, Z. et al. Accelerating materials development for photoelectrochemical hydrogen production: Standards for methods, definitions, and reporting protocols. Journal of Materials Research 25, 3-16, (2010).

220 Wang, J. et al. Facile synthesis of novel $\mathrm{Ag}_{3} \mathrm{PO}_{4}$ tetrapods and the $\{110\}$ facets-dominated photocatalytic activity. CrystEngComm 15, 39-42, (2012). 
221 Yan, S. et al. An Ion Exchange Phase Transformation to $\mathrm{ZnGa}_{2} \mathrm{O}_{4}$ Nanocube Towards Efficient Solar Fuel Synthesis. Advanced Functional Materials 23, 758-763, (2013).

222 Wang, Z. L. Transmission electron microscopy of shape-controlled nanocrystals and their assemblies. J Phys Chem $B$ 104, 1153-1175, (2000).

223 Yang, J., Qi, L., Lu, C., Ma, J. \& Cheng, H. Morphosynthesis of Rhombododecahedral Silver Cages by Self-Assembly Coupled with Precursor Crystal Templating. Angewandte Chemie International Edition 44, 598-603, (2005).

224 Liu, G., Yu, J. C., Lu, G. Q. \& Cheng, H.-M. Crystal facet engineering of semiconductor photocatalysts: motivations, advances and unique properties. Chemical Communications 47, 6763-6783, (2011).

225 Zhou, Z.-Y., Tian, N., Li, J.-T., Broadwell, I. \& Sun, S.-G. Nanomaterials of high surface energy with exceptional properties in catalysis and energy storage. Chemical Society Reviews 40, 41674185, (2011).

226 Chen, C., Ma, W. \& Zhao, J. Semiconductor-mediated photodegradation of pollutants under visible-light irradiation. Chemical Society Reviews 39, 4206-4219, (2010).

227 Martin, D. J. et al. Highly Efficient $\mathrm{H}_{2}$ Evolution from Water under visible light by Structure-Controlled Graphitic Carbon Nitride. Angewandte Chemie International Edition, (2014).

228 Jun, Y.-S. et al. Three-Dimensional Macroscopic Assemblies of LowDimensional Carbon Nitrides for Enhanced Hydrogen Evolution. Angewandte Chemie International Edition 52, 11083-11087, (2013).

229 Jürgens, B. et al. Melem (2,5,8-Triamino-tri-s-triazine), an Important Intermediate during Condensation of Melamine Rings to Graphitic Carbon Nitride: Synthesis, Structure Determination by X-ray Powder Diffractometry, Solid-State NMR, and Theoretical Studies. Journal of the American Chemical Society 125, 1028810300, (2003).

230 Deifallah, M., McMillan, P. F. \& Cora, F. Electronic and Structural Properties of Two-Dimensional Carbon Nitride Graphenes. The Journal of Physical Chemistry C 112, 5447-5453, (2008).

231 Ferrari, A. C. \& Robertson, J. Interpretation of Raman spectra of disordered and amorphous carbon. Physical Review B 61, 1409514107, (2000).

232 Ferrari, A. C., Rodil, S. E. \& Robertson, J. Interpretation of infrared and Raman spectra of amorphous carbon nitrides. Physical Review $B$ 67, 155306, (2003).

233 Larkin, P. J., Makowski, M. P. \& Colthup, N. B. The form of the normal modes of s-triazine: infrared and Raman spectral analysis and ab initio force field calculations. Spectrochimica Acta Part A: Molecular and Biomolecular Spectroscopy 55, 1011-1020, (1999).

234 González, J. F., Román, S., González-García, C. M., Nabais, J. V. \& Ortiz, A. L. Porosity development in activated carbons prepared from walnut shells by carbon dioxide or steam activation. Industrial \& engineering chemistry research 48, 7474-7481, (2009).

235 Zhang, Y., Liu, J., Wu, G. \& Chen, W. Porous graphitic carbon nitride synthesized via direct polymerization of urea for efficient sunlight-driven photocatalytic hydrogen production. Nanoscale 4, 5300-5303, (2012).

236 Zhang, Y., Thomas, A., Antonietti, M. \& Wang, X. Activation of Carbon Nitride Solids by Protonation: Morphology Changes, Enhanced Ionic Conductivity, and Photoconduction Experiments. Journal of the American Chemical Society 131, 50-51, (2008). 
Thomas, A. et al. Graphitic carbon nitride materials: variation of structure and morphology and their use as metal-free catalysts. Journal of materials chemistry 18, 4893-4908, (2008).

$238 \mathrm{Li}$, J. et al. A facile approach to synthesize novel oxygen-doped g$\mathrm{C}_{3} \mathrm{~N}_{4}$ with superior visible-light photoreactivity. Chemical Communications 48, 12017-12019, (2012).

239 Matsumura, M., Saho, Y. \& Tsubomura, H. Photocatalytic hydrogen production from solutions of sulfite using platinized cadmium sulfide powder. The Journal of Physical Chemistry 87, 3807-3808, (1983).

240 Ferreira, K. N., Iverson, T. M., Maghlaoui, K., Barber, J. \& Iwata, S. Architecture of the Photosynthetic Oxygen-Evolving Center. Science 303, 1831-1838, (2004).

241 Patnaik, P. Handbook of Inorganic Chemicals. (McGraw-Hill, 2003).

242 Zheng, H. et al. Nanostructured Tungsten Oxide - Properties, Synthesis, and Applications. Advanced Functional Materials 21, 2175-2196, (2011).

243 Kato, H., Sasaki, Y., Iwase, A. \& Kudo, A. Role of iron ion electron mediator on photocatalytic overall water splitting under visible light irradiation using Z-scheme systems. B Chem Soc Jpn 80, 2457-2464, (2007).

244 Enesca, A., Andronic, L., Duta, A. \& Manolache, S. Optical properties and chemical stability of $\mathrm{WO}_{3}$ and $\mathrm{TiO}_{2}$ thin films photocatalysts. Romanian Journal of Information Science and Technology 10, 269-277, (2007).

245 Ohno, T., Saito, S., Fujihara, K. \& Matsumura, M. Photocatalyzed production of hydrogen and iodine from aqueous solutions of iodide using platinum-loaded $\mathrm{TiO}_{2}$ powder. B Chem Soc Jpn 69, 3059-3064, (1996).

246 González-Borrero, P. P. et al. Optical band-gap determination of nanostructured $\mathrm{WO}_{3}$ film. Appl Phys Lett 96, doi:http://dx.doi.org/10.1063/1061.3313945, (2010).

247 Cabrera, R. Q. et al. Photocatalytic activity of needle-like TiO2/WO3-x thin films prepared by chemical vapour deposition. Journal of Photochemistry and Photobiology A: Chemistry 239, 6064, (2012).

248 Ohno, T., Sarukawa, K. \& Matsumura, M. Crystal faces of rutile and anatase $\mathrm{TiO}_{2}$ particles and their roles in photocatalytic reactions. New journal of chemistry 26, 1167-1170, (2002).

249 Mandzy, N., Grulke, E. \& Druffel, T. Breakage of $\mathrm{TiO}_{2}$ agglomerates in electrostatically stabilized aqueous dispersions. Powder Technology 160, 121-126, (2005).

250 Qu, Y. \& Duan, X. Progress, challenge and perspective of heterogeneous photocatalysts. Chemical Society Reviews 42, 25682580, (2013).

251 Liu, G. et al. A Tantalum Nitride Photoanode Modified with a HoleStorage Layer for Highly Stable Solar Water Splitting. Angewandte Chemie International

Edition, http://dx.doi.org/10.1002/anie.201404697, (2014).

252 Awazu, K. et al. A Plasmonic Photocatalyst Consisting of Silver Nanoparticles Embedded in Titanium Dioxide. Journal of the American Chemical Society 130, 1676-1680, (2008). 